\title{
Transition Probabilities for the Dipole Allowed Fine Structure Transitions in S II
}

\author{
Sultana N. Nahar \\ Department of Astronomy, The Ohio State University, Columbus, Ohio, U.S.A. 43210
}

Received December 14, 1995; accepted in revised form March 6, 1996

\begin{abstract}
The atomic quantities for bound-bound transitions such as the oscillator strengths, the line strengths, and the Einstein $A$-coefficients are obtained for a large number of dipole allowed $(\Delta S=0)$ transitions for the astrophysically important ion SII. The oscillator strengths in $L S$ coupling are obtained in an ab initio manner in the close coupling approximation using R-matrix method. The quantities for the fine structure transitions are obtained through an algebraic transformation of the $L S$ values. The spectroscopic energies of the fine structure levels are used instead of the calculated energies to improve the accuracy of these values. Present calculations employs a 17-state eigenfunction expansion of the S III core states. Result are presented for 1466 fine structure dipole allowed transitions in S II corresponding to 350 transitions in $L S$ coupling. This work reports the first extensive tabulation of S II radiative transitions including fine structure. Comparison is made with the available experimental and theoretical data and good agreement is found with the observed values and other accurate theoretical works.
\end{abstract}

\section{Introduction}

S II is an astrophysically important ion [1], but like most other comparatively larger ions, theoretical studies for the radiative transition probabilities of this ion were carried out only in a small limited scale. However, accurate as well as extensive data are needed for the interpretation of observations being made, such as sulfur emission spectra of the plasma torus of Jupiter's satellite Io by Voyager I and II [2]. Furthermore, collisional rate coefficients have recently been computed for a large number of transitions in S II [3] and it is desirable to complement the atomic datasets for astrophysical modeling with radiative data.

The first extensive work on S II was carried out by Butler et al. [4] under the Opacity Project (OP) [5]. Like all OP calculations, their work was also carried out in $L S$ coupling. But for laboratory plasma experiments and for a variety of astrophysical model applications, it is often the fine structure transitions that are required, rather than the $L S$ values. The purpose of the present work is to provide a reasonably complete set of oscillator strengths ( $f$-values), line strengths ( $S$-values) and the transition rates ( $A$-values) for the dipole allowed fine structure transitions in S II. The method has been applied to several other ions $[6,7]$, and is briefly summerized below.

\section{Summary of the theoretical work and computations}

The calculations for the atomic parameters of the fine structure transitions are based on obtaining the line strength, $S$, in an $a b$ initio manner in the close coupling (CC) approximation. In the $\mathrm{CC}$ approximation the core ion, termed as the "target", is represented by an $N$ electron system. The wavefunction expansion, $\Psi(E)$, for any symmetry, $S L \pi$, of the total $(N+1)$ electron system is represented in terms of the target states as:

$\Psi(E)=A \sum_{i} \chi_{i} \theta_{i}+\sum_{j} c_{j} \Phi_{j}$,

where $\chi_{i}$ is the target ion wave function in a specific state $S_{i} L_{i} \pi_{i}$ and $\theta_{i}$ is the wave function for the $(N+1)$ th electron in a channel labeled as $S_{i} L_{i} \pi_{i} k_{i}^{2} l_{i}(S L \pi) ; k_{i}^{2}$ being its incident kinetic energy. $\Phi_{j}$ 's are the correlation wavefunctions of the $(N+1)$ electron system that compensates the orthogonality condition of the total wavefunction. The $\mathrm{CC}$ sums imply extensive configuration interactions in the coupled wavefunctions for each $S L \pi$. Therefore, provided the relativistic effects are small, the $a b$ initio computations for the derived transition matrix elements should be accurate.

The line strength, $S$, for transition between the initial " $i$ " and the final " $f$ " states is obtained as

$S=\left|\left\langle\Psi_{f}\|D\| \Psi_{i}\right\rangle\right|^{2}$,

where the operator $D$ is

$D=\sum_{n} r_{n}$

in length form, the sum going over total number of electrons in the ion, and $\Psi_{i}, \Psi_{f}$ are the initial and final wavefunctions respectively. The line strength depend on the wavefunctions, not on the energies, of these states. As we expect the present $\mathrm{CC}$ wavefunctions to be very accurate, it is possible to obtain improved oscillator strengths by using observed, rather than calculated, energy differences.

Using the energy difference, $E_{f i}$, of the initial and final states, the oscillator strength, $f_{i f}$, for the transition can be obtained from $S$ as

$f_{i f}=\frac{E_{f i}}{3 g_{i}} S$,

and the Einstein's A-coefficient, $A_{f i}$, as

$A_{f i}($ a.u. $)=\frac{1}{2} \alpha^{3} \frac{g_{i}}{g_{f}} E_{f i}^{2} f_{i f}$,

where $\alpha$ is the fine structure constant, and $g_{i}, g_{f}$ are the statistical weight factors of the initial and final states, respectively. In terms of c.g.s. unit of time,

$A_{f i}\left(s^{-1}\right)=\frac{A_{f i}(\text { a.u. })}{\tau_{0}}$,

where $\tau_{0}=2.4191^{-17} \mathrm{~s}$ is the atomic unit of time. For the present work, the values of $E_{f i}$ are used from the observed energies. 
Table I. The 17 states of SIII employed in the close coupling eigenfunction expansion of $S I I$

\begin{tabular}{llll}
\hline $3 s^{2} 3 p^{2}$ & ${ }^{3} \mathrm{P}^{\mathrm{e}}$ & $3 \mathrm{~s}^{2} 3 \mathrm{p}^{2}$ & ${ }^{1} \mathrm{D}^{\mathrm{e}}$ \\
$3 \mathrm{~s}^{2} 3 \mathrm{p}^{2}$ & ${ }^{1} \mathrm{~S}^{\mathrm{e}}$ & $3 \mathrm{~s} 3 \mathrm{p}^{3}$ & ${ }^{5} \mathrm{~S}^{\circ}$ \\
$3 \mathrm{~s} 3 \mathrm{p}^{3}$ & ${ }^{3} \mathrm{D}^{\circ}$ & $3 \mathrm{~s} 3 \mathrm{p}^{3}$ & ${ }^{3} \mathrm{P}^{\circ}$ \\
$3 \mathrm{~s} 3 \mathrm{p}^{3}$ & ${ }^{1} \mathrm{D}^{\circ}$ & $3 \mathrm{~s}^{2} 3 \mathrm{p} 3 \mathrm{~d}$ & ${ }^{3} \mathrm{~F}^{\circ}$ \\
$3 \mathrm{~s}^{3} \mathrm{p}^{3}$ & ${ }^{1} \mathrm{P}^{\circ}$ & $3 \mathrm{~s} 3 \mathrm{p}^{3}$ & ${ }^{3} \mathrm{~S}^{\circ}$ \\
$3 \mathrm{~s}^{2} 3 \mathrm{p} 3 \mathrm{~d}$ & ${ }^{3} \mathrm{P}^{\circ}$ & $3 \mathrm{~s}^{2} 3 \mathrm{p} 4 \mathrm{~s}$ & ${ }^{3} \mathrm{P}^{\circ}$ \\
$3 \mathrm{~s}^{2} 3 \mathrm{p} 3 \mathrm{~d}$ & ${ }^{3} \mathrm{D}^{\circ}$ & $3 \mathrm{~s}^{2} 3 \mathrm{p} 4 \mathrm{~s}$ & ${ }^{1} \mathrm{P}^{\circ}$ \\
$3 \mathrm{~s}^{2} 3 \mathrm{p} 3 \mathrm{~d}$ & ${ }^{1} \mathrm{D}^{\circ}$ & $3 \mathrm{~s}^{2} 3 \mathrm{p} 3 \mathrm{~d}$ & ${ }^{1} \mathrm{~F}^{\circ}$ \\
$3 \mathrm{~s}^{2} 3 \mathrm{p} 3 \mathrm{~d}$ & ${ }^{1} \mathrm{P}^{\circ}$ & & \\
\hline
\end{tabular}

Spectroscopic configurations: $3 \mathrm{~s}^{2} 3 \mathrm{p}^{2}, 3 \mathrm{~s}^{3} \mathrm{p}^{3}, 3 \mathrm{~s}^{2} 3 \mathrm{p} 3 \mathrm{~d}, 3 \mathrm{~s}^{2} 3 \mathrm{p} 4 \mathrm{~s}$

Correlation configurations: $3 s^{2} p^{2} 3 d, 3 s^{2} 3 d^{2}, 3 p^{4}, 3 p^{3} 3 d, 3 s 3 p 3 d^{2}, 3 p^{3} 4 s$

The fine structure line strengths, $S_{J J}$, are obtained from the LS multiplet strength, $S_{L S}$, through the algebraic transformation as

$S_{J J}=C_{A l}\left(J_{i}, J_{f}\right) S_{L S} /\left[\left(2 S_{i}+1\right)\left(2 L_{i}+1\right)\left(2 L_{f}+1\right)\right]$,

for the allowed transitions $(\Delta J=0, \pm 1) . S_{i}$ is the spin multiplicity which remains the same $\left(S_{i}=S_{f}\right)$ during the transitions. The values of the coefficients $C_{A l}\left(J_{i}, J_{f}\right)$ are obtained from Allen [8]. The $S_{J J}$ values satisfy the condition

$S_{L S}=\sum_{J} S_{J J}$

The fine structure $f$-values, $f_{J J}$, can also be obtained directly from the $L S$ oscillator strengths, $f_{L S}$, as [9]

$$
\begin{aligned}
f_{J J}\left(n_{f} S_{i} L_{f} J_{f}, n_{i} S_{i} L_{i} J_{i}\right)= & f_{L S}\left(n_{f} S_{i} L_{f}, n_{i} S_{i} L_{i}\right)\left(2 J_{f}+1\right) \\
& \times\left(2 L_{i}+1\right) W^{2}\left(L_{f} L_{i} J_{f} J_{i} ; 1 S_{i}\right),
\end{aligned}
$$

where $W\left(L_{f} L_{i} J_{f} J_{i} ; 1 S_{i}\right)$ is a Racah coefficient. The above values also satisfy sum

$$
\begin{aligned}
\sum_{J_{i} J_{f}}\left(2 J_{i}+1\right) f_{J J}\left(n_{f} S_{i} L_{f} J_{f}, n_{i} S_{i} L_{i} J_{i}\right) \\
\quad=\left(2 S_{i}+1\right)\left(2 L_{i}+1\right) f_{L s}\left(n_{f} S_{i} L_{f}, n_{i} S_{i} L_{i}\right)
\end{aligned}
$$

Both algebraic transformations yield fine structure components with about the same accuracy. In the first case the fine structure splitting is carried out for the $L S$ line strengths whereas in the latter case for the $L S f$-values. The first transformation is more convenient to use when observed levels are available. The direct transformation of $f$-values is used

Table II. Absolute measured (expt) and calculated (calc) energies (in Rydberg unit) of $S I I$. The negative sign of the energies are not shown. The degeneracy of the states

\begin{tabular}{|c|c|c|c|c|c|c|c|}
\hline Term & & $E \cdot(\operatorname{expt})$ & $E(\mathrm{calc})$ & Term & & $E(\operatorname{expt})$ & $E$ (calc) \\
\hline $3 s^{2} 3 p^{3}$ & $z^{4} S^{0}$ & 1.721 & 1.761 & $3 s^{2} 3 p^{3}$ & $\mathrm{z}^{2} \mathrm{D}^{0}$ & 1.585 & 1.610 \\
\hline $3 s^{2} 3 p^{3}$ & $\mathrm{z}^{2} \mathrm{P}^{0}$ & 1.497 & 1.517 & $3 s^{3} p^{4}$ & $a^{4} p^{e}$ & 0.995 & 1.034 \\
\hline $3 s 3 p^{4}$ & $a^{2} D^{e}$ & 0.828 & 0.842 & $3 s^{2} 3 p^{2}\left({ }^{3} P\right) 3 d$ & $\mathrm{a}^{2} \mathrm{P}^{\mathrm{e}}$ & 0.757 & 0.773 \\
\hline $3 s^{2} 3 p^{2}\left({ }^{3} P\right) 4 s$ & $b^{4} P^{e}$ & 0.718 & 0.739 & $3 s^{2} 3 p^{2}\left({ }^{3} P\right) 3 d$ & $a^{4} F^{\bullet}$ & 0.714 & 0.727 \\
\hline $3 s^{2} 3 p^{2}\left({ }^{3} P\right) 4 s$ & $b^{2} P^{e}$ & 0.688 & 0.708 & $3 s^{2} 3 p^{2}\left({ }^{3} P\right) 3 d$ & $a^{4} D^{e}$ & 0.680 & 0.696 \\
\hline $3 s^{2} 3 p^{2}\left({ }^{3} p\right) 3 d$ & $a^{2} F^{e}$ & 0.672 & 0.676 & $3 s 3 p^{4}$ & $a^{2} S^{e}$ & 0.629 & 0.632 \\
\hline $3 s^{2} 3 p^{2}\left({ }^{1} D\right) 4 s$ & $b^{2} D^{2}$ & 0.613 & 0.616 & $3 s^{2} 3 p^{2}\left({ }^{3} P\right) 4 p$ & $z^{2} S^{0}$ & 0.577 & 0.574 \\
\hline $3 s^{2} 3 p^{2}\left({ }^{2} D\right) 3 d$ & $a^{2} G^{e}$ & 0.562 & 0.542 & $3 s^{2} 3 p^{2}\left({ }^{3} P\right) 4 p$ & $z^{4} D^{\circ}$ & 0.552 & 0.548 \\
\hline $3 s^{2} 3 p^{2}\left({ }^{3} P\right) 4 p$ & $\mathrm{z}^{4} \mathrm{P}^{\circ}$ & 0.536 & 0.533 & $3 s^{2} 3 p^{2}\left({ }^{3} P\right) 3 d$ & $c^{4} P^{e}$ & 0.529 & 0.524 \\
\hline $3 s^{2} 3 p^{2}\left({ }^{3} P\right) 4 p$ & $\mathrm{y}^{2} \mathrm{D}^{\circ}$ & 0.527 & 0.522 & $3 s^{2} 3 p^{2}\left({ }^{3} p\right) 4 p$ & $y^{4} S^{0}$ & 0.527 & 0.521 \\
\hline $3 s^{2} 3 p^{2}\left({ }^{3} p\right) 4 p$ & $\mathrm{y}^{2} \mathrm{P}^{\circ}$ & 0.505 & 0.499 & $3 s^{2} 3 p^{2}\left({ }^{3} P\right) 3 d$ & $c^{2} D^{e}$ & 0.503 & 0.495 \\
\hline $3 s^{2} 3 p^{2}\left({ }^{1} s\right) 4 s$ & $b^{2} S^{e}$ & 0.478 & 0.477 & $3 s^{2} 3 p^{2}\left({ }^{1} D\right) 3 d$ & $\mathrm{~b}^{2} \mathrm{~F}^{\mathrm{e}}$ & 0.458 & 0.445 \\
\hline $3 \mathrm{~s}^{2} 3 \mathrm{p}^{2}\left({ }^{1} \mathrm{D}\right) 3 \mathrm{~d}$ & $c^{2} p^{e}$ & 0.445 & 0.425 & $3 s^{2} 3 p^{2}\left({ }^{1} D\right) 4 p$ & $\mathrm{z}^{2} \mathrm{~F}^{0}$ & 0.442 & 0.432 \\
\hline $3 s^{2} 3 p^{2}\left({ }^{1} D\right) 4 p$ & $x^{2} D^{\circ}$ & 0.438 & 0.429 & $3 s^{2} 3 p^{2}\left({ }^{1} D\right) 4 p$ & $\mathrm{x}^{2} \mathrm{P}^{0}$ & 0.412 & 0.404 \\
\hline $3 s^{2} 3 p^{2}\left({ }^{1} D\right) 3 d$ & $d^{2} D^{e}$ & 0.408 & 0.384 & $3 s 3 p^{4}$ & $d^{2} P^{e}$ & 0.394 & 0.375 \\
\hline $3 \mathrm{~s}^{2} 3 \mathrm{p}^{2}\left({ }^{1} \mathrm{~s}\right) 3 \mathrm{~d}$ & $\mathrm{e}^{2} \mathrm{D}^{\mathrm{e}}$ & 0.364 & 0.330 & $3 s^{2} 3 p^{2}\left({ }^{3} P\right) 5 s$ & $d^{4} P^{e}$ & 0.347 & 0.347 \\
\hline $3 s^{2} 3 p^{2}\left({ }^{3} P\right) 5 s$ & $e^{2} P^{e}$ & 0.338 & 0.338 & $3 s^{2} 3 p^{2}\left({ }^{1} D\right) 3 d$ & $c^{2} S^{e}$ & 0.339 & 0.320 \\
\hline $3 s^{2} 3 p^{2}\left({ }^{3} P\right) 4 d$ & $b^{4} F^{e}$ & 0.333 & 0.332 & $3 s^{2} 3 p^{2}\left({ }^{3} P\right) 4 d$ & $b^{4} D^{=}$ & 0.324 & 0.324 \\
\hline $3 \mathrm{~s}^{2} 3 \mathrm{p}^{2}\left({ }^{3} \mathrm{P}\right) 4 \mathrm{~d}$ & $e^{4} P^{e}$ & 0.300 & 0.297 & $3 s^{2} 3 p^{2}\left({ }^{3} P\right) 4 d$ & $c^{2} F^{e}$ & 0.296 & 0.292 \\
\hline $3 s^{2} 3 p^{2}\left({ }^{1} s\right) 4 p$ & $w^{2} P^{0}$ & 0.297 & 0.290 & $3 s^{2} 3 p^{2}\left({ }^{3} p\right) 5 p$ & $y^{4} D^{\circ}$ & 0.288 & 0.285 \\
\hline $3 s^{2} 3 p^{2}\left({ }^{3} P\right) 5 p$ & $y^{4} p^{o}$ & 0.282 & 0.281 & $3 s^{2} 3 p^{2}\left({ }^{3} P\right) 5 p$ & $x^{4} S^{0}$ & 0.280 & 0.279 \\
\hline $3 \mathrm{~s}^{2} 3 \mathrm{p}^{2}\left({ }^{3} \mathrm{P}\right) 5 \mathrm{p}$ & $w^{2} D^{0}$ & 0.276 & 0.273 & $3 s^{2} 3 p^{2}\left({ }^{3} P\right) 4 d$ & $f^{2} P^{0}$ & 0.272 & 0.253 \\
\hline $3 s^{2} 3 p^{2}\left({ }^{3} P\right) 4 d$ & $f^{2} D^{e}$ & 0.267 & 0.260 & $3 s^{2} 3 p^{2}\left({ }^{1} D\right) 5 s$ & $\mathrm{~g}^{2} \mathrm{D}^{\mathrm{e}}$ & 0.247 & 0.244 \\
\hline $3 s^{2} 3 p^{2}\left({ }^{1} D\right) 4 d$ & $b^{2} G^{e}$ & 0.224 & 0.220 & $3 s^{2} 3 p^{2}\left({ }^{1} D\right) 4 d$ & $\mathrm{~d}^{2} \mathrm{~F}^{\mathrm{e}}$ & 0.224 & 0.221 \\
\hline $3 s^{2} 3 p^{2}\left({ }^{3} P\right) 6 s$ & $\mathrm{f}^{4} \mathrm{P}^{\mathrm{e}}$ & 0.206 & 0.205 & $3 s^{2} 3 p^{2}\left({ }^{1} D\right) 4 d$ & $\mathrm{~g}^{2} \mathrm{P}^{e}$ & 0.206 & 0.200 \\
\hline $3 s^{2} 3 p^{2}\left({ }^{3} P\right) 6 s$ & $h^{2} P^{e}$ & 0.202 & 0.201 & $3 s^{2} 3 p^{2}\left({ }^{3} P\right) 5 d$ & $c^{4} F^{e}$ & 0.199 & 0.198 \\
\hline $3 s^{2} 3 p^{2}\left({ }^{3} P\right) 5 d$ & $c^{4} D^{e}$ & 0.195 & 0.195 & $3 s^{2} 3 p^{2}\left({ }^{1} D\right) 4 d$ & $\mathrm{~h}^{2} \mathrm{D}^{\mathrm{e}}$ & 0.194 & 0.185 \\
\hline $3 s^{2} 3 p^{2}\left({ }^{3} P\right) 5 d$ & $g^{4} P^{e}$ & 0.187 & 0.185 & $3 s^{2} 3 p^{2}\left({ }^{1} D\right) 5 p$ & $y^{2} F^{o}$ & 0.186 & 0.182 \\
\hline $3 \mathrm{~s}^{2} 3 \mathrm{p}^{2}\left({ }^{1} \mathrm{D}\right) 4 \mathrm{~d}$ & $\mathrm{~d}^{2} \mathrm{~S}^{\mathrm{e}}$ & 0.180 & 0.174 & $3 s^{2} 3 p^{2}\left({ }^{3} P\right) 5 d$ & $e^{2} F^{e}$ & 0.176 & 0.173 \\
\hline $3 \mathrm{~s}^{2} 3 \mathrm{p}^{2}\left({ }^{3} \mathrm{P}\right) 5 \mathrm{~d}$ & $i^{2} p e$ & 0.177 & 0.169 & $3 s^{2} 3 p^{2}\left({ }^{3} P\right) 5 d$ & $i^{2} D^{0}$ & 0.172 & 0.170 \\
\hline $3 s^{2} 3 p^{2}\left({ }^{3} P\right) 7 s$ & $h^{4} P^{e}$ & 0.137 & 0.136 & $3 s^{2} 3 p^{2}\left({ }^{3} P\right) 7 s$ & $j^{2} P^{e}$ & 0.134 & 0.133 \\
\hline $3 s^{2} 3 p^{2}\left({ }^{3} P\right) 6 d$ & $\mathrm{~d}^{4} \mathrm{D}_{*}^{\mathrm{e}}$ & 0.132 & 0.130 & $3 s^{2} 3 p^{2}\left({ }^{3} P\right) 6 d$ & $\mathrm{~d}^{4} \mathrm{~F}_{*}^{\mathrm{e}}$ & 0.130 & 0.132 \\
\hline $3 \mathrm{~s}^{2} 3 \mathrm{p}^{2}\left({ }^{3} \mathrm{P}\right) 6 \mathrm{~d}$ & $\mathrm{i}^{4} \mathrm{Pe}^{*}$ & 0.126 & 0.126 & $3 s^{2} 3 p^{2}\left({ }^{1} D\right) 6 s$ & $\mathrm{j}^{2} \mathrm{D}^{\mathrm{e}}$ & 0.107 & 0.104 \\
\hline $3 s^{2} 3 p^{2}\left({ }^{1} D\right) 5 d$ & $c^{2} G^{e}$ & 0.097 & 0.095 & $3 s^{2} 3 p^{2}\left({ }^{3} P\right) 7 d$ & $\mathrm{e}^{4} \mathrm{D}^{\mathrm{e}} *$ & 0.096 & 0.094 \\
\hline $3 s^{2} 3 p^{2}\left({ }^{3} P\right) 7 d$ & $j^{4} P^{e}$ & 0.091 & 0.091 & $3 \mathrm{~s}^{2} 3 \mathrm{p}^{2}\left({ }^{1} \mathrm{D}\right) 7 \mathrm{~s}$ & $\mathbf{k}^{2} \mathrm{D}^{\mathrm{e}}$ & 0.038 & 0.041 \\
\hline
\end{tabular}
are assinged in ascending order for the even parity and descending order for the odd parity states belonging to the same symmetry. An ast erisk next to a state indicates incomplete set of observed energy levels for the state. 
for the $L S$ multiplets where a complete set of observed fine structure levels is unavailable for one or both the terms, and for transitions between high angular momentum states (transitions higher than $H \leftrightarrow I$ ). Calculated energies are used for these cases.

The lifetime of a state can be obtained easily once the $A$-values of the state are known as

$\tau_{f}=\frac{1}{A_{f}}$

where $A_{f}$ is the total radiative transition probability for the state $f$, i.e.,

$A_{f}=\sum_{i} A_{f i}$.

The present work on $L S$ oscillator strengths for SII is carried out in a similar manner to the OP work [4], but employing a 17-state eigenfunction expansion which inclues the $4 \mathrm{~s}$ orbital (the OP expansion with 15-states does not include the $4 \mathrm{~s}$ orbital); the relevant data for S III are given in Table I. The 17 states, dominated by configurations $3 s^{2} 3 p^{2}, 3 s 3 p^{3}, 3 s^{2} 3 p 3 d$, and $3 s^{2} 3 p 4 s$, of the core ion S III, are optimized using the atomic structure code SUPERSTRUCTURE [10]. The theoretical details of the close coupling approximation using the R-matrix method are described in Ref. [5]. The computations are carried out using the R-matrix codes [11] as developed for the OP [5].

The number of radiative transitions in S II in the present work exceeds that of the previous OP calculations [4], and includes 316 LS bound states below the first ionization threshold and 5002 LS multiplets. A comparison is made of the present calculated energies with the observed ones in Table II. The observed $L S$ energies are obtained through statistical average of the observed fine structure level energies. An asterisk next to a state in the table indicates incomplete set of observed energy levels for the state. The number of observed bound states of S II is smaller, $70 \mathrm{LS}$ terms in total [12], than the number of calculated bound states, 316 . The calculated energies are within $5 \%$ difference of the 65 observed $L S$ energies; the other 5 are within $10 \%$.

For the fine structure transitions the algebraic transformations of the $L S$ values are carried out as described above, with improved accuracy using spectroscopically observed energies, and employing the computer code JJTOLS [7]. Results are obtained for transitions between only those $L S$ states that have been observed, with either complete or incomplete set of observed fine structure levels. Each observed $L S$ term has been designated by a degeneracy symbol, as shown in Table II, and is employed in representing the transitions in this work. This degeneracy assignment may not necessarily match the observed one; an ascending order of alphabets is chosen for the observed even parity states of a symmetry, while a descending order is chosen for the observed odd parity states.

\section{Results and discussion}

The $f$-, $S$, and $A$-values are obtained for a large number of fine structure transitions in SII. These results provide the first extensive set of fine structure transitions in SII. The earlier calculations are mainly for $L S$ transitions and are limited to a small number of transitions except the work by Butler et al. [4].
In order to ascertain the accuracy of the present results, comparison is made with available theoretical and experimental values on SII in Table III. Present $f$-value for the transition $3 s^{2} 3 p^{3}\left(z^{2} D^{o}\right) \rightarrow 3 s^{2} 3 p^{2} 4 s\left(b^{2} D^{e}\right)$ agrees very well with the $f$-value obtained from the beam-foil experiment by Ryan et al. [14] in comparison to other calculations such as by Butler et al. [4] and Ojha and Hibbert [13]. Among the available theoretical calculations, the works of Butler et al. [4] and Ojha and Hibbert [13] are the most elaborate ones, the former using the R-matrix close coupling method and the latter from an optimised configuration-interaction type atomic structure calculation. For the other transition $3 \mathrm{~s}^{2} 3 \mathrm{p}^{3}\left(\mathrm{z}^{2} \mathrm{P}^{\mathrm{o}}\right) \rightarrow 3 \mathrm{~s}^{2} 3 \mathrm{p}^{2} 4 \mathrm{~s}\left(\mathrm{~b}^{2} \mathrm{D}^{\mathrm{e}}\right)$ the present $f$-value is significantly lower than that by Ryan et al. [14]. This experimental $f$-value is much higher than the calculated value by Butler et al. [4] as well indicating a possible overestimation in the measured value. The $f$-value of both Butler et al. [4] and Ojha and Hibbert [13] agree very well with the measured value of Lawrence [15] in pulsed-beam experiment for the transition $\mathrm{z}^{4} \mathrm{~S}^{\circ} \rightarrow \mathrm{a}^{4} \mathrm{P}^{\mathrm{e}}$, whereas the present $f$-value is about $16 \%$ higher. This difference is reflected in the lifetime comparison as well, presented in the lower part of the table, for the fine structure components $3 \mathrm{~s}^{2} 3 \mathrm{p}^{4}\left(\mathrm{a}^{4} \mathrm{P}^{\mathrm{e}}\right)_{5 / 2,3 / 2,1 / 2}$ of the same multiplet obtained from the same measurement by Lawrence [15]. The calculated $f$-value by Cai and Pradhan [3] is lower than the measured value; their calculations were primarily intended for collisional data for S II and not particularly optimized for radiative quantities.

The present $f$-values are compared for a few more transitions with the other calculations, and all theoretical values are found to be in reasonable agreement with each other for the strong transitions. However, Ojha and Hibbert [13] obtain a higher value for the transition $\mathrm{z}^{4} \mathrm{~S}^{0} \rightarrow \mathrm{b}^{4} \mathrm{P}^{\mathrm{e}}$ compared to the other calculations. For the relatively weak transition $\mathrm{z}^{2} \mathrm{P}^{\mathrm{o}} \rightarrow \mathrm{a}^{2} \mathrm{~S}^{\mathrm{e}}$ the present $f$-value agrees with Ojha and Hibbert, while for $\mathrm{z}^{2} \mathrm{P}^{\mathrm{o}} \rightarrow \mathrm{a}^{2} \mathrm{D}^{\mathrm{e}}$ they agree better with

Table III. Comparison of the present $f$-values and lifetimes $(\tau)$.

\begin{tabular}{|c|c|c|c|}
\hline Transition & Present & $\begin{array}{l}f_{i f} \\
\text { Expt. }\end{array}$ & Theo \\
\hline $\mathrm{z}^{2} \mathrm{D}^{\circ} \rightarrow \mathrm{a}^{2} \mathrm{D}^{\mathrm{e}}$ & 0.016 & & $0.011^{b}, 0.011^{c}, 0.012^{e}$ \\
\hline$z^{2} D^{a} \rightarrow b^{2} D^{e}$ & 0.146 & $0.14(0.08)^{\mathrm{a}}$ & $0.156^{\mathrm{b}}, 0.151^{\mathrm{e}}$ \\
\hline$z^{2} D^{\circ} \rightarrow c^{2} D^{e}$ & 0.137 & & $0.164^{b}, 0.145^{c}, 0.164^{e}$ \\
\hline $\mathrm{z}^{2} \mathrm{P}^{0} \rightarrow \mathrm{a}^{2} \mathrm{~S}^{e}$ & 0.008 & & $0.006^{b}, 0.008^{c}$ \\
\hline $\mathrm{z}^{2} \mathrm{P}^{\circ} \rightarrow \mathrm{a}^{2} \mathrm{D}^{\mathrm{e}}$ & 0.0033 & & $0.0011^{b}, 0.00096^{c}, 0.0018^{e}$ \\
\hline$z^{2} P^{o} \rightarrow b^{2} D^{e}$ & 0.042 & $0.20(0.12)^{\mathrm{a}}$ & $0.061^{b}$ \\
\hline$z^{2} P^{o} \rightarrow c^{2} D^{e}$ & 0.305 & & $0.326^{b}, 0.323^{c}$ \\
\hline $\mathrm{z}^{4} \mathrm{~S}^{0} \rightarrow \mathrm{a}^{4} \mathrm{P}^{\mathrm{e}}$ & 0.0377 & $0.032(0.002)^{d}$ & $0.0310^{b}, 0.0329^{c}, 0.0286^{e}$ \\
\hline $\mathrm{z}^{4} \mathrm{~S}^{\circ} \rightarrow \mathrm{b}^{4} \mathrm{P}^{\mathrm{e}}$ & 0.368 & & $0.384^{b}, 0.420^{c}, 0.383^{e}$ \\
\hline \multirow[t]{2}{*}{$\mathrm{z}^{4} \mathrm{~S}^{0} \rightarrow \mathrm{c}^{4} \mathrm{P}^{\mathrm{e}}$} & 2.39 & & $2.43^{b}, 2.262^{c}, 2.246^{c}$ \\
\hline & & $\tau(\mathrm{ns})$ & \\
\hline State & Present & Expt. & Theo. \\
\hline $\mathrm{a}^{4} \mathrm{P}^{6} 5 / 2$ & 18.99 & $22.4(2)^{\mathrm{d}}$ & \\
\hline$a^{4} P^{5} 3 / 2$ & 18.73 & $22.4(2)^{\mathrm{d}}$ & \\
\hline $\mathrm{a}^{4} \mathrm{P}^{\mathrm{e}} 1 / 2$ & 18.59 & $21.3(2)^{d}$ & \\
\hline
\end{tabular}

a Ryan et al. (1989).

b Butler et al. (OP).

c Ojha and Hibbert (1989).

¿ Lawrence (1969)

- Cai and Pradhan (1993). 
the CC calculations of Butler et al.; the present $f$-value is higher than the other calculations.

The complete set of $f-, S$ - and $A$-values for the dipole allowed fine structure transitions in SII obtained in the present work is presented in Table IV. The first line of each subset of data in the table corresponds to the $L S$ transition, followed by the fine structure components. The degeneracy labels of the $L S$ terms in the table correspond to those assigned in Table II for the energies. The energies of the initial and final levels in the fine structure set are given in unit of $\mathrm{cm}^{-1}$, while the initial and the final $L S$ states and the transitional energy differences are given in Rydberg units. The $A$-values are given in $\mathrm{s}^{-1}$. An asterisk $\left(^{*}\right)$ below an $L S$ term in Table IV indicates an incomplete set of observed energy levels, and an asterisk for the energy corresponding to a transition indicates one or both of the levels missing from the set of observed energies. The table contains 1466 fine structure transitions corresponding to 350 in $L S$ coupling.

The results obtained in the present work correspond to the 1466 fine structure transitions among the 70 observed LS bound states of S II. This is comparatively a small fraction of the total number of possible fine structure transitions that can be processed among 316 calculated $L S$ bound states.

Based on accuracy of the calculated energies and comparison with other works, the accuracy of the present $f-, S$ and $A$-values should be within 15-20\% for most of the transitions. It should however be noted that the present method obtains the fine structure components through pure algebraic transformation and does not consider any relativistic mixing of $L S$ terms explicitly in the wavefunctions. This may result in higher uncertainty for transitions between bighly excited levels where $L S J$-mixing is significant. In that case the intercombination line could be strong enough to reduce the strengths of some of the dipole allowed lines, or shift the strengths among some fine structure components.

\section{Conclusion}

Radiative transition probabilities for a large number of transitions in S II are presented. Observed energies are used in the transformation of the $L S$ coupled transition matrix elements to fine structure for improved accuracy. As there are only a few experimental oscillator strengths available, a comprehensive evaluation of the theoretical data is difficult; however there is good agreement with previous accurate theoretical calculations. The overall uncertainty due to relativistic effects for the dipole transitions should be small. However the transitions among highly excited levels may have higher uncertainty due to neglected mixing effects. Present results should provide a reasonably complete set of data for a large number of fine structure transitions in S II.

The entire table of transition probabilities and energies, Table IV, is available in electronic form from the author at: nahar@seaton.mps.ohio-state.edu, (a FORTRAN77 code is attached to the table to read the $A$-values and calculate the lifetimes).

\section{Acknowledgements}

I would like to thank Professor Anil K. Pradhan for comments and suggestions. This work was supported by NASA grants NAGW-3315 and NAS32643. The computational work was carried out on the Cray YMP at the Ohio Supercomputer Center.

\section{References}

1. Osterbrock, D. E., "Astrophysics of Gaseous Nebulae and Active Galactic Nuclei" (University Science Books, Mill Valley, CA 1989).

2. Moos, W. et al., Astrophys. J. 382, L105 (1991).

3. Wei Cai and Pradhan, A. K., Astrophys. J. Suppl. 88, 329 (1993).

4. Butler, K., Mendoza, C. and Zeippen, C.J., (to be published); data is . presently stored in the Opacity Project database, TOPbase, at CDS.

5. Seaton, M. J., J. Phys. B20, 6363 (1987).

6. Nahar, S. N., Physica Scripta 48, 297 (1993).

7. Nahar, S. N., Astron. Astrophys. 293, 6363 (1995).

8. Allen, C. W., "Astrophysical Quantities" (3rd Edition) (Athlone Press, London 1976).

9. Seaton, M. J., Yu, Y., Mihalas, D. and Pradhan, A. K., Mon. Not. R. Astron. Soc. 266, 805 (1994).

10. Eissner, W., Jones, M. and Nussbaumer, H., Comput. Phys. Commun. 8, $270(1974)$

11. Berrington, K. A. et al., J. Phys. B20, 6379 (1987).

12. Martin, W. C., Zalubas, R., and Musgrove, A., J. Phys. Chem. Ref. Data 19, 821 (1990); Kaufman, V. and Martin, W. C., J. Phys. Chem. Ref. Data 22, 279 (1993).

13. Ojha, P. C. and Hibbert, A., J. Phys. B22, 1153 (1989).

14. Ryan, L. J., Rayburn, L. A. and Cunningham, A. J., J. Quant. Spectrosc. Radiat. Transfer 42, 295 (1989).

15. Lawrence, G. M., Phys. Rev. 179, 134 (1969). 
Table IV. The f-, $S$ - and A-values for transitions in $S I I$

\begin{tabular}{|c|c|c|c|c|c|c|c|c|}
\hline Transition & $\begin{array}{l}E_{i} \\
\mathrm{~cm}^{-1}\end{array}$ & $\begin{array}{l}E_{f} \\
\mathrm{~cm}^{-1} \\
\end{array}$ & $\begin{array}{l}E_{f i} \\
R y\end{array}$ & $g_{i}$ & $g_{f}$ & $f_{i f}$ & $s$ & $\begin{array}{l}A_{f_{i}} \\
\mathrm{~s}^{-1} \\
\end{array}$ \\
\hline \multirow[t]{4}{*}{$\mathrm{z}^{4} \mathrm{~S}^{\circ} \rightarrow \mathrm{a}^{4} \mathrm{P}^{\mathbf{e}}$} & 1.7207 & 0.9952 & $7.255 \mathrm{E}-01$ & 4 & 12 & $3.768 \mathrm{E}-02$ & $6.232 E-01$ & $5.309 \mathrm{E}+07$ \\
\hline & 0.000 & 79395.390 & $7.235 E-01$ & 4 & 6 & $1.879 \mathrm{E}-02$ & $3.116 \mathrm{E}-01$ & $5.266 \mathrm{E}+07$ \\
\hline & 0.000 & 79756.830 & $7.268 \mathrm{E}-01$ & 4 & 4 & $1.258 \mathrm{E}-02$ & $2.077 \mathrm{E}-01$ & $5.338 \mathrm{E}+07$ \\
\hline & 0.000 & 79962.610 & $7.287 \mathrm{E}-01$ & 4 & 2 & $6.307 \mathrm{E}-03$ & $1.039 E-01$ & $5.380 E+07$ \\
\hline \multirow{4}{*}{$\mathrm{z}^{4} \mathrm{~S}^{\mathrm{o}} \rightarrow \mathrm{b}^{4} \mathrm{P}^{\mathrm{e}}$} & 1.7207 & 0.7183 & $1.002 E+00$ & 4 & 12 & $3.677 \mathrm{E}-01$ & $4.402 \mathrm{E}+00$ & $9.893 \mathrm{E}+08$ \\
\hline & 0.000 & 110268.600 & $1.005 E+00$ & 4 & 6 & $1.843 \mathrm{E}-01$ & $2.201 E+00$ & $9.963 \mathrm{E}+08$ \\
\hline & 0.000 & 109831.590 & $1.001 \mathrm{E}+00$ & 4 & 4 & $1.224 \mathrm{E}-01$ & $1.467 \mathrm{E}+00$ & $9.848 \mathrm{E}+08$ \\
\hline & 0.000 & 109560.690 & $9.984 \mathrm{E}-01$ & 4 & 2 & $6.104 \mathrm{E}-02$ & $7.336 \mathrm{E}-01$ & $9.774 \mathrm{E}+08$ \\
\hline \multirow{4}{*}{$z^{4} S^{0} \rightarrow c^{4} P^{e}$} & 1.7207 & 0.5294 & $1.191 E+00$ & 4 & 12 & $2.392 \mathrm{E}+\infty 0$ & $2.409 \mathrm{E}+01$ & $9.088 \mathrm{E}+09$ \\
\hline & 0.000 & 130602.210 & $1.190 \mathrm{E}+00$ & 4 & 6 & $1.195 \mathrm{E}+\infty$ & $1.205 \mathrm{E}+01$ & $9.060 \mathrm{E}+09$ \\
\hline & 0.000 & 130818.850 & $1.192 \mathrm{E}+00$ & 4 & 4 & $7.977 \mathrm{E}-01$ & $8.030 \mathrm{E}+00$ & $9.106 \mathrm{E}+09$ \\
\hline & 0.000 & 130948.940 & $1.193 E+00$ & 4 & 2 & $3.993 \mathrm{E}-01$ & $4.015 \mathrm{E}+00$ & $9.133 \mathrm{E}+09$ \\
\hline \multirow{4}{*}{$\mathrm{z}^{4} \mathrm{~S}^{\circ} \rightarrow \mathrm{d}^{4} \mathrm{P}^{\mathrm{e}}$} & 1.7207 & 0.3473 & $1.373 E+\infty 0$ & 4 & 12 & $4.469 \mathrm{E}-02$ & $3.905 \mathrm{E}-01$ & $2.257 \mathrm{E}+08$ \\
\hline & 0.000 & 150996.410 & $1.376 \mathrm{E}+00$ & 4 & 6 & $2.239 \mathrm{E}-02$ & $1.952 \mathrm{E}-01$ & $2.270 \mathrm{E}+08$ \\
\hline & 0.000 & 150531.310 & $1.372 \mathrm{E}+00$ & 4 & 4 & $1.488 \mathrm{E}-02$ & $1.302 \mathrm{E}-01$ & $2.249 \mathrm{E}+08$ \\
\hline & 0.000 & 150258.510 & $1.369 \mathrm{E}+00$ & 4 & 2 & $7.426 \mathrm{E}-03$ & $6.508 \mathrm{E}-02$ & $2.237 \mathrm{E}+08$ \\
\hline \multirow{4}{*}{$z^{4} S^{0} \rightarrow e^{4} P^{e}$} & 1.7207 & 0.2996 & $1.421 E+\infty 0$ & 4 & 12 & $5.048 \mathrm{E}-01$ & $4.262 \mathrm{E}+00$ & $2.729 \mathrm{E}+09$ \\
\hline & 0.000 & 155818.710 & $1.420 \mathrm{E}+00$ & 4 & 6 & $2.522 \mathrm{E}-01$ & $2.131 \mathrm{E}+00$ & $2.722 \mathrm{E}+09$ \\
\hline & 0.000 & 156029.540 & $1.422 \mathrm{E}+00$ & 4 & 4 & $1.683 \mathrm{E}-01$ & $1.421 \mathrm{E}+00$ & $2.733 \mathrm{E}+09$ \\
\hline & 0.000 & 156148.480 & $1.423 \mathrm{E}+00$ & 4 & 2 & $8.423 \mathrm{E}-02$ & $7.104 \mathrm{E}-01$ & $2.740 \mathrm{E}+09$ \\
\hline \multirow[t]{4}{*}{$z^{4} S^{0} \rightarrow f^{4} P^{e}$} & 1.7207 & 0.2063 & $1.514 \mathrm{E}+00$ & 4 & 12 & $1.435 \mathrm{E}-02$ & $1.137 \mathrm{E}-01$ & $8.814 \mathrm{E}+07$ \\
\hline & 0.000 & 166479.820 & $1.517 \mathrm{E}+\infty 0$ & 4 & 6 & $7.190 \mathrm{E}-03$ & $5.687 \mathrm{E}-02$ & $8.861 E+07$ \\
\hline & 0.000 & 165991.350 & $1.513 \mathrm{E}+\infty 0$ & 4 & 4 & $4.779 \mathrm{E}-03$ & $3.791 \mathrm{E}-02$ & $8.782 \mathrm{E}+07$ \\
\hline & 0.000 & 165719.230 & $1.510 \mathrm{E}+00$ & 4 & 2 & $2.386 \mathrm{E}-03$ & $1.896 \mathrm{E}-02$ & $8.739 E+07$ \\
\hline \multirow[t]{4}{*}{$z^{4} S^{\circ} \rightarrow g^{4} P^{\circ}$} & 1.7207 & 0.1868 & $1.534 \mathrm{E}+00$ & 4 & 12 & $1.613 \mathrm{E}-01$ & $1.262 \mathrm{E}+00$ & $1.016 \mathrm{E}+09$ \\
\hline & 0.000 & 168217.100 & $1.533 \mathrm{E}+00$ & 4 & 6 & $8.061 \mathrm{E}-02$ & $6.311 \mathrm{E}-01$ & $1.014 \mathrm{E}+09$ \\
\hline & 0.000 & 168397.890 & $1.535 \mathrm{E}+00$ & 4 & 4 & $5.380 \mathrm{E}-02$ & $4.207 \mathrm{E}-01$ & $1.018 \mathrm{E}+09$ \\
\hline & 0.000 & 168490.430 & $1.535 \mathrm{E}+\infty 0$ & 4 & 2 & $2.692 \mathrm{E}-02$ & $2.104 \mathrm{E}-01$ & $1.019 E+09$ \\
\hline \multirow[t]{4}{*}{$\mathrm{z}^{4} \mathrm{~S}^{\circ} \rightarrow \mathrm{h}^{4} \mathrm{P}^{\mathrm{e}}$} & 1.7207 & 0.1369 & $1.584 \mathrm{E}+\infty 0$ & 4 & 12 & $6.870 \mathrm{E}-03$ & $5.205 \mathrm{E}-02$ & $4.614 \mathrm{E}+07$ \\
\hline & 0.000 & 174098.100 & $1.587 \mathrm{E}+\infty 0$ & 4 & 6 & $3.441 E-03$ & $2.603 \mathrm{E}-02$ & $4.638 \mathrm{E}+07$ \\
\hline & 0.000 & 173595.770 & $1.582 \mathrm{E}+00$ & 4 & 4 & $2.287 \mathrm{E}-03$ & $1.735 \mathrm{E}-02$ & $4.597 \mathrm{E}+07$ \\
\hline & 0.000 & 173316.830 & $1.579 \mathrm{E}+00$ & 4 & 2 & $1.142 E-03$ & $8.675 \mathrm{E}-03$ & $4.576 \mathrm{E}+07$ \\
\hline \multirow[t]{4}{*}{$z^{4} S^{\circ} \rightarrow i^{4} P^{e}$} & 1.7207 & 0.1263 & $1.594 \mathrm{E}+00$ & 4 & 12 & $7.119 \mathrm{E}-02$ & $5.358 \mathrm{E}-01$ & $4.845 \mathrm{E}+08$ \\
\hline & 0.000 & 174892.750 & $1.594 \mathrm{E}+00$ & 4 & 6 & $3.558 \mathrm{E}-02$ & $2.679 \mathrm{E}-01$ & $4.839 \mathrm{E}+08$ \\
\hline & 0.000 & 175019.780 & $1.595 \mathrm{E}+00$ & 4 & 4 & $2.374 \mathrm{E}-02$ & $1.786 \mathrm{E}-01$ & $4.850 \mathrm{E}+08$ \\
\hline & 0.000 & 175083.510 & $1.596 \mathrm{E}+00$ & 4 & 2 & $1.187 \mathrm{E}-02$ & $8.930 \mathrm{E}-02$ & $4.855 \mathrm{E}+08$ \\
\hline \multirow[t]{4}{*}{$z^{4} S^{0} \rightarrow j^{4} P^{e}$} & 1.7207 & 0.0905 & $1.630 \mathrm{E}+00$ & 4 & 12 & $3.734 \mathrm{E}-02$ & $2.749 \mathrm{E}-01$ & $2.657 \mathrm{E}+08$ \\
\hline & 0.000 & 178848.960 & $1.630 \mathrm{E}+\infty 0$ & 4 & 6 & $1.867 \mathrm{E}-02$ & $1.374 \mathrm{E}-01$ & $2.655 \mathrm{E}+08$ \\
\hline & 0.000 & 178915.200 & $1.630 \mathrm{E}+\infty 0$ & 4 & 4 & $1.245 \mathrm{E}-02$ & $9.162 \mathrm{E}-02$ & $2.658 \mathrm{E}+08$ \\
\hline & 0.000 & 178969.320 & $1.631 \mathrm{E}+\infty$ & 4 & 2 & $6.226 \mathrm{E}-03$ & $4.581 \mathrm{E}-02$ & $2.660 \mathrm{E}+08$ \\
\hline \multirow[t]{4}{*}{$y^{4} S^{o} \rightarrow d^{4} P^{e}$} & 0.5267 & 0.3473 & $1.794 \mathrm{E}-01$ & 4 & 12 & $3.311 \mathrm{E}-01$ & $2.215 \mathrm{E}+01$ & $2.854 \mathrm{E}+07$ \\
\hline & 131028.850 & 150996.410 & $1.820 \mathrm{E}-01$ & 4 & 6 & $1.679 E-01$ & $1.107 \mathrm{E}+01$ & $2.979 \mathrm{E}+07$ \\
\hline & 131028.850 & 150531.310 & $1.777 \mathrm{E}-01$ & 4 & 4 & $1.093 \mathrm{E}-01$ & $7.382 \mathrm{E}+00$ & $2.773 \mathrm{E}+07$ \\
\hline & 131028.850 & 150258.510 & $1.753 \mathrm{E}-01$ & 4 & 2 & $5.392 \mathrm{E}-02$ & $3.691 \mathrm{E}+00$ & $2.662 \mathrm{E}+07$ \\
\hline \multirow[t]{4}{*}{$\mathrm{y}^{4} \mathrm{~S}^{0} \rightarrow \mathrm{e}^{4} \mathrm{P}^{\mathrm{e}}$} & 0.5267 & 0.2996 & $2.270 \mathrm{E}-01$ & 4 & 12 & $7.840 \mathrm{E}-01$ & $4.144 \mathrm{E}+01$ & $1.082 \mathrm{E}+08$ \\
\hline & 131028.850 & 155818.710 & $2.259 \mathrm{E}-01$ & 4 & 6 & $3.900 \mathrm{E}-01$ & $2.072 \mathrm{E}+01$ & $1.066 \mathrm{E}+08$ \\
\hline & 131028.850 & 156029.540 & $2.278 \mathrm{E}-01$ & 4 & 4 & $2.622 \mathrm{E}-01$ & $1.381 \mathrm{E}+01$ & $1.093 E+08$ \\
\hline & 131028.850 & 156148.480 & $2.289 \mathrm{E}-01$ & 4 & 2 & $1.317 \mathrm{E}-01$ & $6.907 \mathrm{E}+00$ & $1.109 E+08$ \\
\hline
\end{tabular}


Table IV. Continued

\begin{tabular}{|c|c|c|c|c|c|c|c|c|}
\hline Transition & $\begin{array}{l}E_{i} \\
\mathrm{~cm}^{-1}\end{array}$ & $\begin{array}{l}E_{f} \\
\mathrm{~cm}^{-1}\end{array}$ & $\begin{array}{l}E_{f i} \\
\mathrm{Ry}\end{array}$ & $g_{i}$ & $g_{f}$ & $f_{i f}$ & $S$ & $\begin{array}{l}A_{f i} \\
\mathrm{~s}^{-1}\end{array}$ \\
\hline \multirow[t]{4}{*}{$y^{4} S^{\circ} \rightarrow f^{4} P^{e}$} & 0.5267 & 0.2063 & $3.204 \mathrm{E}-01$ & 4 & 12 & $2.612 \mathrm{E}-02$ & $9.782 \mathrm{E}-01$ & $7.179 E+06$ \\
\hline & 131028.850 & 166479.820 & $3.231 \mathrm{E}-01$ & 4 & 6 & $1.317 \mathrm{E}-02$ & $4.891 \mathrm{E}-01$ & $7.361 \mathrm{E}+06$ \\
\hline & 131028.850 & 165991.350 & $3.186 \mathrm{E}-01$ & 4 & 4 & $8.657 \mathrm{E}-03$ & $3.261 \mathrm{E}-1$ & $7.058 \mathrm{E}+06$ \\
\hline & 131028.850 & 165719.230 & $3.161 \mathrm{E}-01$ & 4 & 2 & $4.294 \mathrm{E}-03$ & $1.630 \mathrm{E}-01$ & $6.893 \mathrm{E}+06$ \\
\hline \multirow{4}{*}{$y^{4} S^{0} \rightarrow g^{4} p^{e}$} & 0.5267 & 0.1868 & $3.398 \mathrm{E}-01$ & 4 & 12 & $1.099 E-01$ & $3.880 \mathrm{E}+00$ & $3.398 \mathrm{E}+07$ \\
\hline & 131028.850 & 168217.100 & $3.389 \mathrm{E}-01$ & 4 & 6 & $5.478 \mathrm{E}-02$ & $1.940 \mathrm{E}+00$ & $3.369 \mathrm{E}+07$ \\
\hline & 131028.850 & 168397.890 & $3.406 \mathrm{E}-01$ & 4 & 4 & $3.671 \mathrm{E}-02$ & $1.293 \mathrm{E}+00$ & $3.420 \mathrm{E}+07$ \\
\hline & 131028.850 & 168490.430 & $3.414 \mathrm{E}-01$ & 4 & 2 & $1.840 \mathrm{E}-02$ & $6.466 \mathrm{E}-01$ & $3.444 \mathrm{E}+07$ \\
\hline \multirow{4}{*}{$y^{4} S^{o} \rightarrow b^{4} P^{e}$} & 0.5267 & 0.1369 & $3.898 \mathrm{E}-01$ & 4 & 12 & $7.897 \mathrm{E}-03$ & $2.431 \mathrm{E}-01$ & $3.212 \mathrm{E}+06$ \\
\hline & 131028.850 & 174098.100 & $3.925 \mathrm{E}-01$ & 4 & 6 & $3.976 \mathrm{E}-03$ & $1.216 \mathrm{E}-01$ & $3.280 \mathrm{E}+06$ \\
\hline & 131028.850 & 173595.770 & $3.879 \mathrm{E}-01$ & 4 & 4 & $2.620 \mathrm{E}-03$ & $8.104 \mathrm{E}-02$ & $3.166 \mathrm{E}+06$ \\
\hline & 131028.850 & 173316.830 & $3.854 \mathrm{E}-01$ & 4 & 2 & $1.301 E-03$ & $4.052 \mathrm{E}-02$ & $3.105 \mathrm{E}+06$ \\
\hline \multirow[t]{4}{*}{$\mathrm{y}^{4} \mathrm{~S}^{\circ} \rightarrow \mathrm{i}^{4} \mathrm{P}^{\mathrm{e}}$} & 0.5267 & 0.1263 & $4.004 \mathrm{E}-01$ & 4 & 12 & $3.424 \mathrm{E}-02$ & $1.026 \mathrm{E}+\infty 0$ & $1.470 \mathrm{E}+07$ \\
\hline & 131028.850 & 174892.750 & $3.997 \mathrm{E}-01$ & 4 & 6 & $1.709 \mathrm{E}-02$ & $5.130 \mathrm{E}-01$ & $1.462 \mathrm{E}+07$ \\
\hline & 131028.850 & 175019.780 & $4.009 \mathrm{E}-01$ & 4 & 4 & $1.143 E-02$ & $3.420 \mathrm{E}-01$ & $1.475 \mathrm{E}+07$ \\
\hline & 131028.850 & 175083.510 & $4.015 \mathrm{E}-01$ & 4 & 2 & $5.722 \mathrm{E}-03$ & $1.710 \mathrm{E}-01$ & $1.482 \mathrm{E}+07$ \\
\hline \multirow[t]{4}{*}{$y^{4} S^{0} \rightarrow j^{4} P^{6}$} & 0.5267 & 0.0905 & $4.362 \mathrm{E}-01$ & 4 & 12 & $1.510 \mathrm{E}-02$ & $4.155 \mathrm{E}-01$ & $7.692 \mathrm{E}+06$ \\
\hline & 131028.850 & 178848.960 & $4.358 \mathrm{E}-01$ & 4 & 6 & $7.545 \mathrm{E}-03$ & $2.078 \mathrm{E}-01$ & $7.673 E+06$ \\
\hline & 131028.850 & 178915.200 & $4.364 E-01$ & 4 & 4 & $5.037 \mathrm{E}-03$ & $1.385 \mathrm{E}-01$ & $7.705 E+06$ \\
\hline & 131028.850 & 178969.320 & $4.369 \mathrm{E}-01$ & 4 & 2 & $2.521 \mathrm{E}-03$ & $6.925 \mathrm{E}-02$ & $7.731 \mathrm{E}+06$ \\
\hline \multirow[t]{4}{*}{$x^{4} S^{\circ} \rightarrow f^{4} P^{0}$} & 0.2798 & 0.2063 & $7.355 \mathrm{E}-02$ & 4 & 12 & $3.921 \mathrm{E}-01$ & $6.397 \mathrm{E}+01$ & $5.679 \mathrm{E}+06$ \\
\hline & 158118.750 & 166479.820 & $7.620 \mathrm{E}-02$ & 4 & 6 & $2.031 \mathrm{E}-01$ & $3.198 \mathrm{E}+01$ & $6.315 \mathrm{E}+06$ \\
\hline & 158118.750 & 165991.350 & $7.170 \mathrm{E}-02$ & 4 & 4 & $1.274 \mathrm{E}-01$ & $2.132 \mathrm{E}+01$ & $5.261 \mathrm{E}+06$ \\
\hline & 158118.750 & 165719.230 & $6.920 \mathrm{E}-02$ & 4 & 2 & $6.148 \mathrm{E}-02$ & $1.066 \mathrm{E}+01$ & $4.729 E+06$ \\
\hline \multirow[t]{4}{*}{$x^{4} S^{0} \rightarrow g^{4} P^{e}$} & 0.2798 & 0.1868 & $9.299 \mathrm{E}-02$ & 4 & 12 & $9.294 \mathrm{E}-01$ & $1.199 \mathrm{E}+02$ & $2.152 \mathrm{E}+07$ \\
\hline & 158118.750 & 168217.100 & $9.200 \mathrm{E}-02$ & 4 & 6 & $4.598 \mathrm{E}-01$ & $5.997 \mathbf{E}+01$ & $2.084 \mathrm{E}+07$ \\
\hline & 158118.750 & 168397.890 & $9.370 \mathrm{E}-02$ & 4 & 4 & $3.122 \mathrm{E}-01$ & $3.998 \mathrm{E}+01$ & $2.201 \mathrm{E}+07$ \\
\hline & 158118.750 & 168490.430 & $9.450 \mathrm{E}-02$ & 4 & 2 & $1.574 \mathrm{E}-01$ & $1.999 \mathrm{E}+01$ & $2.258 \mathrm{E}+07$ \\
\hline \multirow[t]{4}{*}{$\mathrm{x}^{4} \mathrm{~S}^{0} \rightarrow \mathrm{h}^{4} \mathrm{P}^{0}$} & 0.2798 & 0.1369 & $1.429 \mathrm{E}-01$ & 4 & 12 & $4.042 \mathrm{E}-02$ & $3.394 \mathrm{E}+00$ & $2.210 \mathrm{E}+06$ \\
\hline & 158118.750 & 174098.100 & $1.456 \mathrm{E}-01$ & 4 & 6 & $2.059 \mathrm{E}-02$ & $1.697 \mathrm{E}+\infty 0$ & $2.337 \mathrm{E}+06$ \\
\hline & 158118.750 & 173595.770 & $1.410 \mathrm{E}-01$ & 4 & 4 & $1.329 \mathrm{E}-02$ & $1.131 \mathrm{E}+\infty$ & $2.123 \mathrm{E}+06$ \\
\hline & 158118.750 & 173316.830 & $1.385 E-01$ & 4 & 2 & $6.528 \mathrm{E}-03$ & $5.656 \mathrm{E}-01$ & $2.012 \mathrm{E}+06$ \\
\hline \multirow[t]{4}{*}{$\mathrm{x}^{4} \mathrm{~S}^{\circ} \rightarrow \mathrm{i}^{4} \mathrm{P}^{\mathrm{e}}$} & 0.2798 & 0.1263 & $1.535 \mathrm{E}-01$ & 4 & 12 & $1.634 \mathrm{E}-01$ & $1.277 \mathrm{E}+01$ & $1.031 \mathrm{E}+07$ \\
\hline & 158118.750 & 174892.750 & $1.528 \mathrm{E}-01$ & 4 & 6 & $8.132 \mathrm{E}-02$ & $6.387 \mathrm{E}+\infty 0$ & $1.017 \mathrm{E}+07$ \\
\hline & 158118.750 & 175019.780 & $1.540 \mathrm{E}-01$ & 4 & 4 & $5.464 \mathrm{E}-02$ & $4.258 \mathrm{E}+\infty 0$ & $1.041 \mathrm{E}+07$ \\
\hline & 158118.750 & 175083.510 & $1.546 \mathrm{E}-01$ & 4 & 2 & $2.743 \mathrm{E}-02$ & $2.129 \mathrm{E}+\infty 0$ & $1.053 \mathrm{E}+07$ \\
\hline \multirow[t]{4}{*}{$x^{4} S^{o} \rightarrow j^{4} P^{e}$} & 0.2798 & 0.0905 & $1.893 \mathrm{E}-01$ & 4 & 12 & $6.073 \mathrm{E}-02$ & $3.850 \mathrm{E}+\infty 0$ & $5.826 \mathrm{E}+06$ \\
\hline & 158118.750 & 178848.960 & $1.889 \mathrm{E}-01$ & 4 & 6 & $3.030 \mathrm{E}-02$ & $1.925 \mathrm{E}+\infty 0$ & $5.790 \mathrm{E}+06$ \\
\hline & 158118.750 & 178915.200 & $1.895 \mathrm{E}-01$ & 4 & 4 & $2.027 \mathrm{E}-02$ & $1.283 E+\infty 0$ & $5.845 \mathrm{E}+06$ \\
\hline & 158118.750 & 178969.320 & $1.900 \mathrm{E}-01$ & 4 & 2 & $1.016 \mathrm{E}-02$ & $6.416 \mathrm{E}-01$ & $5.892 \mathrm{E}+06$ \\
\hline \multirow[t]{4}{*}{$\mathrm{a}^{4} \mathrm{P}^{\mathrm{e}} \rightarrow \mathrm{y}^{4} \mathrm{~S}^{\circ}$} & 0.9952 & 0.5267 & $4.686 \mathrm{E}-01$ & 12 & 4 & $1.225 \mathrm{E}-02$ & $9.408 \mathrm{E}-01$ & $6.478 E+07$ \\
\hline & 79395.390 & 131028.850 & $4.705 \mathrm{E}-01$ & 6 & 4 & $1.230 \mathrm{E}-02$ & $4.704 \mathrm{E}-01$ & $3.279 E+07$ \\
\hline & 79756.830 & 131028.850 & $4.672 \mathrm{E}-01$ & 4 & 4 & $1.221 \mathrm{E}-02$ & $3.136 \mathrm{E}-01$ & $2.141 E+07$ \\
\hline & 79962.610 & 131028.850 & $4.653 \mathrm{E}-01$ & 2 & 4 & $1.216 \mathrm{E}-02$ & $1.568 \mathrm{E}-01$ & $1.058 \mathrm{E}+07$ \\
\hline \multirow[t]{4}{*}{$\mathrm{a}^{4} \mathrm{P}^{\mathrm{e}} \rightarrow \mathrm{x}^{4} \mathrm{~S}^{0}$} & 0.9952 & 0.2798 & $7.154 \mathrm{E}-01$ & 12 & 4 & $9.552 \mathrm{E}-03$ & $4.807 \mathrm{E}-01$ & $1.178 \mathrm{E}+08$ \\
\hline & 79395.390 & 158118.750 & $7.174 \mathrm{E}-01$ & 6 & 4 & $9.578 \mathrm{E}-03$ & $2.403 E-01$ & $5.939 \mathrm{E}+07$ \\
\hline & 79756.830 & 158118.750 & $7.141 \mathrm{E}-01$ & 4 & 4 & $9.534 \mathrm{E}-03$ & $1.602 \mathrm{E}-01$ & $3.905 E+07$ \\
\hline & 79962.610 & 158118.750 & $7.122 \mathrm{E}-01$ & 2 & 4 & $9.509 \mathrm{E}-03$ & $8.011 \mathrm{E}-02$ & $1.937 \mathrm{E}+07$ \\
\hline
\end{tabular}


Table IV. Continued

\begin{tabular}{|c|c|c|c|c|c|c|c|c|}
\hline Transition & $\begin{array}{l}E_{i} \\
\mathrm{~cm}^{-1}\end{array}$ & $\begin{array}{l}E_{f} \\
\mathrm{~cm}^{-1}\end{array}$ & $\begin{array}{l}E_{f i} \\
\mathrm{Ry}\end{array}$ & $g_{i}$ & $g_{f}$ & $f_{i f}$ & $S$ & $\begin{array}{l}A_{f i} \\
\mathrm{~s}^{-1}\end{array}$ \\
\hline \multirow[t]{8}{*}{$\mathrm{a}^{4} \mathrm{P}^{\circ} \rightarrow \mathrm{z}^{4} \mathrm{P}^{\circ}$} & 0.9952 & 0.5362 & $4.590 \mathrm{E}-01$ & 12 & 12 & $1.556 \mathrm{E}-02$ & $1.221 \mathrm{E}+00$ & $2.634 \mathrm{E}+07$ \\
\hline & 79395.390 & 130134.160 & $4.624 \mathrm{E}-01$ & 6 & 6 & $1.097 \mathrm{E}-02$ & $4.272 \mathrm{E}-01$ & $1.885 \mathrm{E}+07$ \\
\hline & 79395.390 & 129858.180 & $4.599 \mathrm{E}-01$ & 6 & 4 & $4.678 \mathrm{E}-03$ & $1.831 E-01$ & $1.192 \mathrm{E}+07$ \\
\hline & 79756.830 & 130134.160 & $4.591 \mathrm{E}-01$ & 4 & 6 & $7.005 E-03$ & $1.831 \mathrm{E}-01$ & $7.906 \mathrm{E}+06$ \\
\hline & 79756.830 & 129858.180 & $4.566 \mathrm{E}-01$ & 4 & 4 & $2.064 \mathrm{E}-03$ & $5.425 \mathrm{E}-02$ & $3.457 \mathrm{E}+06$ \\
\hline & 79756.830 & 129787.830 & $4.559 \mathrm{E}-01$ & 4 & 2 & $6.441 E-03$ & $1.695 \mathrm{E}-01$ & $2.150 \mathrm{E}+07$ \\
\hline & 79962.610 & 129858.180 & $4.547 \mathrm{E}-01$ & 2 & 4 & $1.285 \mathrm{E}-02$ & $1.695 \mathrm{E}-01$ & $1.067 \mathrm{E}+07$ \\
\hline & 79962.610 & 129787.830 & $4.540 \mathrm{E}-01$ & 2 & 2 & $2.566 \mathrm{E}-03$ & $3.391 \mathrm{E}-02$ & $4.248 \mathrm{E}+06$ \\
\hline \multirow[t]{8}{*}{$\mathrm{a}^{4} \mathrm{P}^{\mathrm{e}} \rightarrow \mathrm{y}^{4} \mathrm{P}^{\circ}$} & 0.9952 & 0.2825 & $7.127 \mathrm{E}-01$ & 12 & 12 & $3.626 \mathrm{E}-03$ & $1.831 \mathrm{E}-01$ & $1.480 \mathrm{E}+07$ \\
\hline & 79395.390 & 158038.600 & $7.167 \mathrm{E}-01$ & 6 & 6 & $2.552 \mathrm{E}-03$ & $6.410 \mathrm{E}-02$ & $1.053 E+07$ \\
\hline & 79395.390 & 157579.680 & $7.125 \mathrm{E}-01$ & 6 & 4 & $1.087 \mathrm{E}-03$ & $2.747 \mathrm{E}-02$ & $6.651 \mathrm{E}+06$ \\
\hline & 79756.830 & 158038.600 & $7.134 \mathrm{E}-01$ & 4 & 6 & $1.633 \mathrm{E}-03$ & $2.747 \mathrm{E}-02$ & $4.451 \mathrm{E}+06$ \\
\hline & 79756.830 & 157579.680 & $7.092 \mathrm{E}-01$ & 4 & 4 & $4.811 \mathrm{E}-04$ & $8.140 \mathrm{E}-03$ & $1.943 \mathrm{E}+06$ \\
\hline & 79756.830 & 157677.320 & $7.101 \mathrm{E}-01$ & 4 & 2 & $1.505 \mathrm{E}-03$ & $2.544 \mathrm{E}-02$ & $1.219 \mathrm{E}+07$ \\
\hline & 79962.610 & 157579.680 & $7.073 \mathrm{E}-01$ & 2 & 4 & $2.999 \mathrm{E}-03$ & $2.544 \mathrm{E}-02$ & $6.025 \mathrm{E}+06$ \\
\hline & 79962.610 & 157677.320 & $7.082 \mathrm{E}-01$ & 2 & 2 & $6.005 \mathrm{E}-04$ & $5.087 \mathrm{E}-03$ & $2.419 \mathrm{E}+06$ \\
\hline \multirow[t]{9}{*}{$a^{4} P^{e} \rightarrow z^{4} D^{\circ}$} & 0.9952 & 0.5517 & $4.436 \mathrm{E}-01$ & 12 & 20 & $4.526 \mathrm{E}-05$ & $3.674 \mathrm{E}-03$ & $4.292 \mathrm{E}+04$ \\
\hline & 79395.390 & 128599.160 & $4.484 \mathrm{E}-01$ & 6 & 8 & $3.661 \mathrm{E}-05$ & $1.469 E-03$ & $4.434 \mathrm{E}+04$ \\
\hline & 79395.390 & 128233.200 & $4.450 \mathrm{E}-01$ & 6 & 6 & $8.174 E-06$ & $3.306 \mathrm{E}-04$ & $1.300 \mathrm{E}+04$ \\
\hline & 79395.390 & 127976.340 & $4.427 \mathrm{E}-01$ & 6 & 4 & $9.035 \mathrm{E}-07$ & $3.674 \mathrm{E}-05$ & $2.133 E+03$ \\
\hline & 79756.830 & 128233.200 & $4.417 \mathrm{E}-01$ & 4 & 6 & $2.840 E-05$ & $7.714 \mathrm{E}-04$ & $2.967 \mathrm{E}+04$ \\
\hline & 79756.830 & 127976.340 & $4.394 \mathrm{E}-01$ & 4 & 4 & $1.435 \mathrm{E}-05$ & $3.918 \mathrm{E}-04$ & $2.225 \mathrm{E}+04$ \\
\hline & 79756.830 & 127825.080 & $4.380 \mathrm{E}-01$ & 4 & 2 & $2.235 \mathrm{E}-06$ & $6.123 \mathrm{E}-05$ & $6.887 \mathrm{E}+03$ \\
\hline & 79962.610 & 127976.340 & $4.375 E-01$ & 2 & 4 & $2.232 \mathrm{E}-05$ & $3.061 \mathrm{E}-04$ & $1.716 \mathrm{E}+04$ \\
\hline & 79962.610 & 127825.080 & $4.361 \mathrm{E}-01$ & 2 & 2 & $2.225 \mathrm{E}-05$ & $3.061 \mathrm{E}-04$ & $3.400 \mathrm{E}+04$ \\
\hline \multirow[t]{9}{*}{$a^{4} P^{\circ} \rightarrow y^{4} D^{\circ}$} & 0.9952 & 0.2878 & $7.075 \mathrm{E}-01$ & 12 & 20 & $5.889 \mathrm{E}-04$ & $2.997 \mathrm{E}-02$ & $1.421 \mathrm{E}+06$ \\
\hline & 79395.390 & 157558.770 & $7.123 \mathrm{E}-01$ & 6 & 8 & $4.744 E-04$ & $1.199 \mathrm{E}-02$ & $1.450 \mathrm{E}+06$ \\
\hline & 79395.390 & 157173.690 & $7.088 \mathrm{E}-01$ & 6 & 6 & $1.062 \mathrm{E}-04$ & $2.697 \mathrm{E}-03$ & $4.286 \mathrm{E}+05$ \\
\hline & 79395.390 & 156939.500 & $7.066 \mathrm{E}-01$ & 6 & 4 & $1.176 \mathrm{E}-05$ & $2.997 \mathrm{E}-04$ & $7.077 \mathrm{E}+04$ \\
\hline & 79756.830 & 157173.690 & $7.055 \mathrm{E}-01$ & 4 & 6 & $3.700 \mathrm{E}-0.4$ & $6.293 \mathrm{E}-03$ & $9.861 \mathrm{E}+05$ \\
\hline & 79756.830 & 156939.500 & $7.033 \mathrm{E}-01$ & 4 & 4 & $1.873 E-04$ & $3.197 \mathrm{E}-03$ & $7.443 E+05$ \\
\hline & 79756.830 & 156829.750 & $7.023 \mathrm{E}-01$ & 4 & 2 & $2.923 E-05$ & $4.995 \mathrm{E}-04$ & $2.316 \mathrm{E}+05$ \\
\hline & 79962.610 & 156939.500 & $7.014 \mathrm{E}-01$ & 2 & 4 & $2.919 \mathrm{E}-04$ & $2.497 \mathrm{E}-03$ & $5.769 \mathrm{E}+05$ \\
\hline & 79962.610 & 156829.750 & $7.004 \mathrm{E}-01$ & 2 & 2 & $2.915 \mathrm{E}-04$ & $2.497 \mathrm{E}-03$ & $1.149 \mathrm{E}+06$ \\
\hline \multirow[t]{4}{*}{$b^{4} P^{e} \rightarrow y^{4} S^{0}$} & 0.7183 & 0.5267 & $1.916 \mathrm{E}-01$ & 12 & 4 & $1.384 \mathrm{E}-01$ & $2.601 \mathrm{E}+01$ & $1.224 \mathrm{E}+08$ \\
\hline & 110268.600 & 131028.850 & $1.892 \mathrm{E}-01$ & 6 & 4 & $1.367 \mathrm{E}-01$ & $1.300 \mathrm{E}+01$ & $5.895 \mathrm{E}+07$ \\
\hline & 109831.590 & 131028.850 & $1.931 \mathrm{E}-01$ & 4 & 4 & $1.395 \mathrm{E}-01$ & $8.670 \mathrm{E}+00$ & $4.178 \mathrm{E}+07$ \\
\hline & 109560.690 & 131028.850 & $1.956 \mathrm{E}-01$ & 2 & 4 & $1.413 \mathrm{E}-01$ & $4.335 \mathrm{E}+00$ & $2.172 \mathrm{E}+07$ \\
\hline \multirow[t]{4}{*}{$\mathrm{b}^{4} \mathrm{P}^{\mathrm{e}} \rightarrow \mathrm{x}^{4} \mathrm{~S}^{0}$} & 0.7183 & 0.2798 & $4.384 \mathrm{E}-01$ & 12 & 4 & $1.534 \mathrm{E}-03$ & $1.259 \mathrm{E}-01$ & $7.104 \mathrm{E}+06$ \\
\hline & 110268.600 & 158118.750 & $4.361 \mathrm{E}-01$ & 6 & 4 & $1.525 \mathrm{E}-03$ & $6.296 \mathrm{E}-02$ & $3.495 \mathrm{E}+06$ \\
\hline & 109831.590 & 158118.750 & $4.400 \mathrm{E}-01$ & 4 & 4 & $1.539 \mathrm{E}-03$ & $4.197 \mathrm{E}-02$ & $2.393 \mathrm{E}+06$ \\
\hline & 109560.690 & 158118.750 & $4.425 \mathrm{E}-01$ & 2 & 4 & $1.548 \mathrm{E}-03$ & $2.099 \mathrm{E}-02$ & $1.217 \mathrm{E}+06$ \\
\hline \multirow[t]{8}{*}{$\mathrm{b}^{4} \mathrm{P}^{\mathrm{e}} \rightarrow \mathrm{z}^{4} \mathrm{P}^{\mathrm{o}}$} & 0.7183 & 0.5362 & $1.821 \mathrm{E}-01$ & 12 & 12 & $3.255 \mathrm{E}-01$ & $6.437 \mathrm{E}+01$ & $8.668 \mathrm{E}+07$ \\
\hline & 110268.600 & 130134.160 & $1.811 \mathrm{E}-01$ & 6 & 6 & $2.267 \mathrm{E}-01$ & $2.253 \mathrm{E}+01$ & $5.971 \mathrm{E}+07$ \\
\hline & 110268.600 & 129858.180 & $1.786 \mathrm{E}-01$ & 6 & 4 & $9.580 \mathrm{E}-02$ & $9.655 \mathrm{E}+00$ & $3.682 \mathrm{E}+07$ \\
\hline & 109831.590 & 130134.160 & $1.850 \mathrm{E}-01$ & 4 & 6 & $1.489 \mathrm{E}-01$ & $9.655 \mathrm{E}+00$ & $2.728 \mathrm{E}+07$ \\
\hline & 109831.590 & 129858.180 & $1.825 \mathrm{E}-01$ & 4 & 4 & $4.351 \mathrm{E}-02$ & $2.861 \mathrm{E}+00$ & $1.164 \mathrm{E}+07$ \\
\hline & 109831.590 & 129787.830 & $1.818 \mathrm{E}-01$ & 4 & 2 & $1.354 \mathrm{E}-01$ & $8.940 \mathrm{E}+00$ & $7.191 \mathrm{E}+07$ \\
\hline & 109560.690 & 129858.180 & $1.850 \mathrm{E}-01$ & 2 & 4 & $2.757 \mathrm{E}-01$ & $8.940 \mathrm{E}+00$ & $3.789 \mathrm{E}+07$ \\
\hline & 109560.690 & 129787.830 & $1.843 \mathrm{E}-01$ & 2 & 2 & $5.492 \mathrm{E}-02$ & $1.788 \mathrm{E}+00$ & $1.499 \mathrm{E}+07$ \\
\hline \multirow[t]{8}{*}{$b^{4} P^{\circ} \rightarrow y^{4} P^{o}$} & 0.7183 & 0.2825 & $4.358 \mathrm{E}-01$ & 12 & 12 & $1.577 \mathrm{E}-03$ & $1.303 \mathrm{E}-01$ & $2.406 \mathrm{E}+06$ \\
\hline & 110268.600 & 158038.600 & $4.354 \mathrm{E}-01$ & 6 & 6 & $1.103 \mathrm{E}-03$ & $4.561 \mathrm{E}-02$ & $1.680 \mathrm{E}+06$ \\
\hline & 110268.600 & 157579.680 & $4.312 \mathrm{E}-01$ & 6 & 4 & $4.683 \mathrm{E}-04$ & $1.955 \mathrm{E}-02$ & $1.049 \mathrm{E}+06$ \\
\hline & 109831.590 & 158038.600 & $4.393 \mathrm{E}-01$ & 4 & 6 & $7.156 \mathrm{E}-04$ & $1.955 \mathrm{E}-02$ & $7.395 \mathrm{E}+05$ \\
\hline & 109831.590 & 157579.680 & $4.351 \mathrm{E}-01$ & 4 & 4 & $2.100 \mathrm{E}-04$ & $5.792 \mathrm{E}-03$ & $3.193 \mathrm{E}+05$ \\
\hline & 109831.590 & 157677.320 & $4.360 \mathrm{E}-01$ & 4 & 2 & $6.576 \mathrm{E}-0.4$ & $1.810 \mathrm{E}-02$ & $2.008 \mathrm{E}+06$ \\
\hline & 109560.690 & 157579.680 & $4.376 \mathrm{E}-01$ & 2 & 4 & $1.320 \mathrm{E}-03$ & $1.810 \mathrm{E}-02$ & $1.015 \mathrm{E}+06$ \\
\hline & 109560.690 & 157677.320 & $4.385 \mathrm{E}-01$ & 2 & 2 & $2.646 \mathrm{E}-04$ & $3.620 \mathrm{E}-03$ & $4.086 \mathrm{E}+05$ \\
\hline
\end{tabular}


Table IV. Continued

\begin{tabular}{|c|c|c|c|c|c|c|c|c|}
\hline Transition & $\begin{array}{l}E_{i} \\
\mathrm{~cm}^{-1} \\
\end{array}$ & $\begin{array}{l}E_{f} \\
\mathrm{~cm}^{-1} \\
\end{array}$ & $\begin{array}{l}E_{f i} \\
\text { Ry }\end{array}$ & $g_{i}$ & $g_{f}$ & $f_{i f}$ & $S$ & $\begin{array}{l}A_{f i} \\
\mathrm{~s}^{-1} \\
\end{array}$ \\
\hline \multirow{9}{*}{$\mathrm{b}^{4} \mathrm{P}^{\mathrm{c}} \rightarrow \mathrm{z}^{4} \mathrm{D}^{\circ}$} & 0.7183 & 0.5517 & $1.666 \mathrm{E}-01$ & 12 & 20 & $5.429 \mathrm{E}-01$ & $1.173 \mathrm{E}+02$ & $7.263 E+07$ \\
\hline & 110268.600 & 128599.160 & $1.671 \mathrm{E}-01$ & 6 & 8 & $4.357 \mathrm{E}-01$ & $4.693 \mathrm{E}+01$ & $7.328 \mathrm{E}+07$ \\
\hline & 110268.600 & 128233.200 & $1.637 \mathrm{E}-01$ & 6 & 6 & $9.603 \mathrm{E}-02$ & $1.056 \mathrm{E}+01$ & $2.067 \mathrm{E}+07$ \\
\hline & 110268.600 & 127976.340 & $1.614 \mathrm{E}-01$ & 6 & 4 & $1.052 \mathrm{E}-02$ & $1.173 \mathrm{E}+00$ & $3.302 \mathrm{E}+06$ \\
\hline & 109831.590 & 128233.200 & $1.676 \mathrm{E}-01$ & 4 & 6 & $3.441 \mathrm{E}-01$ & $2.464 \mathrm{E}+01$ & $5.176 \mathrm{E}+07$ \\
\hline & 109831.590 & 127976.340 & $1.653 \mathrm{E}-01$ & 4 & 4 & $1.724 \mathrm{E}-01$ & $1.251 \mathrm{E}+01$ & $3.783 \mathrm{E}+07$ \\
\hline & 109831.590 & 127825.080 & $1.639 \mathrm{E}-01$ & 4 & 2 & $2.671 \mathrm{E}-02$ & $1.955 \mathrm{E}+00$ & $1.153 \mathrm{E}+07$ \\
\hline & 109560.690 & 127976.340 & $1.678 \mathrm{E}-01$ & 2 & 4 & $2.734 \mathrm{E}-01$ & $9.777 \mathrm{E}+00$ & $3.092 \mathrm{E}+07$ \\
\hline & 109560.690 & 127825.080 & $1.664 \mathrm{E}-01$ & 2 & 2 & $2.712 \mathrm{E}-01$ & $9.777 \mathrm{E}+00$ & $6.031 \mathrm{E}+07$ \\
\hline \multirow{9}{*}{$b^{4} P^{\circ} \rightarrow y^{4} D^{\circ}$} & 0.7183 & 0.2878 & $4.305 \mathrm{E}-01$ & 12 & 20 & $3.474 \mathrm{E}-04$ & $2.905 \mathrm{E}-02$ & $3.102 \mathrm{E}+05$ \\
\hline & 110268.600 & 157558.770 & $4.310 \mathrm{E}-01$ & 6 & 8 & $2.782 \mathrm{E}-04$ & $1.162 \mathrm{E}-02$ & $3.113 \mathrm{E}+05$ \\
\hline & 110268.600 & 157173.690 & $4.275 \mathrm{E}-01$ & 6 & 6 & $6.209 E-05$ & $2.614 \mathrm{E}-03$ & $9.114 \mathrm{E}+04$ \\
\hline & 110268.600 & 156939.500 & $4.253 \mathrm{E}-01$ & 6 & 4 & $6.863 \mathrm{E}-06$ & $2.905 \mathrm{E}-04$ & $1.496 \mathrm{E}+04$ \\
\hline & 109831.590 & 157173.690 & $4.314 \mathrm{E}-01$ & 4 & 6 & $2.193 \mathrm{E}-04$ & $6.100 \mathrm{E}-03$ & $2.185 \mathrm{E}+05$ \\
\hline & 109831.590 & 156939.500 & $4.292 \mathrm{E}-01$ & 4 & 4 & $1.108 \mathrm{E}-04$ & $3.098 \mathrm{E}-03$ & $1.640 \mathrm{E}+05$ \\
\hline & 109831.590 & 156829.750 & $4.282 \mathrm{E}-01$ & 4 & 2 & $1.727 \mathrm{E}-05$ & $4.841 \mathrm{E}-04$ & $5.088 \mathrm{E}+04$ \\
\hline & 109560.690 & 156939.500 & $4.317 \mathrm{E}-01$ & 2 & 4 & $1.742 \mathrm{E}-04$ & $2.421 \mathrm{E}-03$ & $1.304 \mathrm{E}+05$ \\
\hline & 109560.690 & 156829.750 & $4.307 E-01$ & 2 & 2 & $1.738 \mathrm{E}-04$ & $2.421 \mathrm{E}-03$ & $2.589 \mathrm{E}+05$ \\
\hline \multirow[t]{4}{*}{$\mathrm{c}^{4} \mathrm{P}^{\mathrm{e}} \rightarrow \mathrm{y}^{4} \mathrm{~S}^{\circ}$} & 0.5294 & 0.5267 & $2.703 E-03$ & 12 & 4 & $2.137 \mathrm{E}-03$ & $2.846 \mathrm{E}+01$ & $3.763 \mathrm{E}+02$ \\
\hline & 130602.210 & 131028.850 & $3.900 \mathrm{E}-03$ & 6 & 4 & $3.083 E-03$ & $1.423 E+01$ & $5.650 \mathrm{E}+02$ \\
\hline & 130818.850 & 131028.850 & $1.900 \mathrm{E}-03$ & 4 & 4 & $1.502 \mathrm{E}-03$ & $9.486 \mathrm{E}+\infty$ & $4.355 \mathrm{E}+01$ \\
\hline & 130948.940 & 131028.850 & $7.000 \mathrm{E}-04$ & 2 & 4 & $5.534 \mathrm{E}-04$ & $4.743 \mathrm{E}+00$ & $1.089 \mathrm{E}+00$ \\
\hline \multirow[t]{4}{*}{$c^{4} P^{c} \rightarrow x^{4} S^{0}$} & 0.5294 & 0.2798 & $2.496 \mathrm{E}-01$ & 12 & 4 & $4.634 \mathrm{E}-03$ & $6.684 \mathrm{E}-01$ & $6.954 \mathrm{E}+06$ \\
\hline & 130602.210 & 158118.750 & $2.508 \mathrm{E}-01$ & 6 & 4 & $4.657 \mathrm{E}-03$ & $3.342 \mathrm{E}-01$ & $3.529 E+06$ \\
\hline & 130818.850 & 158118.750 & $2.488 \mathrm{E}-01$ & 4 & 4 & $4.620 \mathrm{E}-03$ & $2.228 \mathrm{E}-01$ & $2.297 \mathrm{E}+06$ \\
\hline & 130948.940 & 158118.750 & $2.476 \mathrm{E}-01$ & 2 & 4 & $4.597 \mathrm{E}-03$ & $1.114 \mathrm{E}-01$ & $1.132 \mathrm{E}+06$ \\
\hline \multirow[t]{8}{*}{$c^{4} \mathrm{P}^{6} \rightarrow \mathrm{y}^{4} \mathrm{P}^{0}$} & 0.5294 & 0.2825 & $2.469 \mathrm{E}-01$ & 12 & 12 & $5.359 \mathrm{E}-03$ & $7.814 \mathrm{E}-01$ & $2.624 \mathrm{E}+06$ \\
\hline & 130602.210 & 158038.600 & $2.501 \mathrm{E}-01$ & 6 & 6 & $3.800 \mathrm{E}-03$ & $2.735 \mathrm{E}-01$ & $1.909 \mathrm{E}+06$ \\
\hline & 1306012.210 & 157579.680 & $2.459 \mathrm{E}-01$ & 6 & 4 & $1.601 E-03$ & $1.172 \mathrm{E}-01$ & $1.167 E+06$ \\
\hline & 130818.850 & 158038.600 & $2.481 \mathrm{E}-01$ & 4 & 6 & $2.423 E-03$ & $1.172 \mathrm{E}-01$ & $7.987 \mathrm{E}+05$ \\
\hline & 130818.850 & 157579.680 & $2.439 \mathrm{E}-01$ & 4 & 4 & $7.058 \mathrm{E}-04$ & $3.473 \mathrm{E}-02$ & $3.373 \mathrm{E}+05$ \\
\hline & 130818.850 & 157677.320 & $2.448 \mathrm{E}-01$ & 4 & 2 & $2.214 \mathrm{E}-03$ & $1.085 \mathrm{E}-01$ & $2.131 \mathrm{E}+06$ \\
\hline & 130948.940 & 157579.680 & $2.427 \mathrm{E}-01$ & 2 & 4 & $4.390 \mathrm{E}-03$ & $1.085 \mathrm{E}-01$ & $1.038 \mathrm{E}+06$ \\
\hline & 130948.940 & 157677.320 & $2.436 \mathrm{E}-01$ & 2 & 2 & $8.812 \mathrm{E}-04$ & $2.171 \mathrm{E}-02$ & $4.200 \mathrm{E}+05$ \\
\hline \multirow[t]{9}{*}{$c^{4} P^{e} \rightarrow y^{4} D^{\circ}$} & 0.5294 & 0.2878 & $2.416 \mathrm{E}-01$ & 12 & 20 & $2.829 \mathrm{E}-03$ & $4.216 \mathrm{E}-01$ & $7.961 \mathrm{E}+05$ \\
\hline & 130602.210 & 157558.770 & $2.457 \mathrm{E}-01$ & 6 & 8 & $2.302 \mathrm{E}-03$ & $1.686 \mathrm{E}-01$ & $8.371 \mathrm{E}+05$ \\
\hline & 130602.210 & 157173.690 & $2.422 \mathrm{E}-01$ & 6 & 6 & $5.105 \mathrm{E}-04$ & $3.794 \mathrm{E}-02$ & $2,406 \mathrm{E}+05$ \\
\hline & 130602.210 & 156939.500 & $2.400 \mathrm{E}-01$ & 6 & 4 & $5.621 \mathrm{E}-05$ & $4.216 \mathrm{E}-03$ & $3.901 E+04$ \\
\hline & 130818.850 & 157173.690 & $2.402 \mathrm{E}-01$ & 4 & 6 & $1.772 \mathrm{E}-03$ & $8.853 \mathrm{E}-02$ & $5.475 \mathrm{E}+05$ \\
\hline & 130818.850 & 156939.500 & $2.380 \mathrm{E}-01$ & 4 & 4 & $8.919 \mathrm{E}-04$ & $4.497 \mathrm{E}-02$ & $4.058 \mathrm{E}+05$ \\
\hline & 130818.850 & 156829.750 & $2.370 \mathrm{E}-01$ & 4 & 2 & $1.388 \mathrm{E}-04$ & $7.026 \mathrm{E}-03$ & $1.252 \mathrm{E}+05$ \\
\hline & 130948.940 & 156939.500 & $2.368 \mathrm{E}-01$ & 2 & 4 & $1.387 \mathrm{E}-03$ & $3.513 \mathrm{E}-02$ & $3.122 \mathrm{E}+05$ \\
\hline & 130948.940 & 156829.750 & $2.358 \mathrm{E}-01$ & 2 & 2 & $1.381 \mathrm{E}-03$ & $3.513 \mathrm{E}-02$ & $6.166 \mathrm{E}+05$ \\
\hline \multirow[t]{4}{*}{$\mathrm{d}^{4} \mathrm{P}^{\mathrm{e}} \rightarrow \mathrm{x}^{4} \mathrm{~S}^{\circ}$} & 0.3473 & 0.2798 & $6.744 \mathrm{E}-02$ & 12 & 4 & $2.029 E-01$ & $1.083 E+02$ & $2.224 \mathrm{E}+07$ \\
\hline & 150996.410 & 158118.750 & $6.490 \mathrm{E}-02$ & 6 & 4 & $1.953 \mathrm{E}-01$ & $5.417 \mathrm{E}+01$ & $9.911 E+06$ \\
\hline & 150531.310 & 158118.750 & $6.920 \mathrm{E}-02$ & 4 & 4 & $2.082 \mathrm{E}-01$ & $3.611 \mathrm{E}+01$ & $8.010 \mathrm{E}+06$ \\
\hline & 150258.510 & 158118.750 & $7.160 \mathrm{E}-02$ & 2 & 4 & $2.155 \mathrm{E}-01$ & $1.806 \mathrm{E}+01$ & $4.436 \mathrm{E}+06$ \\
\hline \multirow[t]{8}{*}{$d^{4} P^{c} \rightarrow y^{4} P^{o}$} & 0.3473 & 0.2825 & $6.476 \mathrm{E}-02$ & 12 & 12 & $5.009 \mathrm{E}-01$ & $2.784 \mathrm{E}+02$ & $1.687 \mathrm{E}+07$ \\
\hline & 150996.410 & 158038.600 & $6.420 \mathrm{E}-02$ & 6 & 6 & $3.475 \mathrm{E}-01$ & $9.744 E+01$ & $1.151 E+07$ \\
\hline & 150996.410 & 157579.680 & $6.000 \mathrm{E}-02$ & 6 & 4 & $1.392 \mathrm{E}-01$ & $4.176 \mathrm{E}+01$ & $6.038 \mathrm{E}+06$ \\
\hline & 150531.310 & 158038.600 & $6.850 \mathrm{E}-02$ & 4 & 6 & $2.384 \mathrm{E}-01$ & $4.176 \mathrm{E}+01$ & $5.990 \mathrm{E}+06$ \\
\hline & 150531.310 & 157579.680 & $6.430 \mathrm{E}-02$ & 4 & 4 & $6.630 \mathrm{E}-02$ & $1.237 \mathrm{E}+01$ & $2.202 \mathrm{E}+06$ \\
\hline & 150531.310 & 157677.320 & $6.520 \mathrm{E}-02$ & 4 & 2 & $2.101 \mathrm{E}-01$ & $3.867 \mathrm{E}+01$ & $1.435 \mathrm{E}+07$ \\
\hline & 150258.510 & 157579.680 & $6.670 \mathrm{E}-02$ & 2 & 4 & $4.299 E-01$ & $3.867 \mathrm{E}+01$ & $7.680 \mathrm{E}+06$ \\
\hline & 150258.510 & 157677.320 & $6.760 \mathrm{E}-02$ & 2 & 2 & $8.713 E-02$ & $7.734 \mathrm{E}+00$ & $3.198 \mathrm{E}+06$ \\
\hline
\end{tabular}


Table IV. Continued

\begin{tabular}{|c|c|c|c|c|c|c|c|c|}
\hline Transition & $\begin{array}{l}E_{i} \\
\mathrm{~cm}^{-1}\end{array}$ & $\begin{array}{l}E_{f} \\
\mathrm{~cm}^{-1}\end{array}$ & $\begin{array}{l}E_{f i} \\
\mathrm{Ry}\end{array}$ & $g_{i}$ & $g_{f}$ & $f_{i f}$ & $s$ & $\begin{array}{l}A_{f i} \\
\mathrm{~s}^{-1} \\
\end{array}$ \\
\hline \multirow[t]{9}{*}{$d^{4} P^{e} \rightarrow y^{4} D^{0}$} & 0.3473 & 0.2878 & $5.949 \mathrm{E}-02$ & 12 & 20 & $8.396 \mathrm{E}-01$ & $5.081 \mathrm{E}+02$ & $1.432 \mathrm{E}+07$ \\
\hline & 150996.410 & 157558.770 & $5.980 \mathrm{E}-02$ & 6 & 8 & $6.752 \mathrm{E}-01$ & $2.032 \mathrm{E}+02$ & $1.454 \mathrm{E}+07$ \\
\hline & 150996.410 & 157173.690 & $5.630 \mathrm{E}-02$ & 6 & 6 & $1.430 \mathrm{E}-01$ & $4.573 \mathrm{E}+01$ & $3.641 E+06$ \\
\hline & 150996.410 & 156939.500 & $5.410 \mathrm{E}-02$ & 6 & 4 & $1.527 \mathrm{E}-02$ & $5.081 \mathrm{E}+00$ & $5.385 \mathrm{E}+05$ \\
\hline & 150531.310 & 157173.690 & $6.060 \mathrm{E}-02$ & 4 & 6 & $5.388 \mathrm{E}-01$ & $1.067 \mathrm{E}+02$ & $1.060 \mathrm{E}+07$ \\
\hline & 150531.310 & 156939.500 & $5.840 \mathrm{E}-02$ & 4 & 4 & $2.637 \mathrm{E}-01$ & $5.419 \mathrm{E}+01$ & $7.225 \mathrm{E}+06$ \\
\hline & 150531.310 & 156829.750 & $5.740 \mathrm{E}-02$ & 4 & 2 & $4.050 \mathrm{E}-02$ & $8.468 \mathrm{E}+00$ & $2.144 \mathrm{E}+06$ \\
\hline & 150258.510 & 156939.500 & $6.080 \mathrm{E}+02$ & 2 & 4 & $4.290 \mathrm{E}-01$ & $4.234 \mathrm{E}+01$ & $6.369 \mathrm{E}+06$ \\
\hline & 150258.510 & 156829.750 & $5.980 \mathrm{E}-02$ & 2 & 2 & $4.220 \mathrm{E}-01$ & $4.234 \mathrm{E}+01$ & $1.212 \mathrm{E}+07$ \\
\hline \multirow[t]{4}{*}{$e^{4} P^{6} \rightarrow x^{4} S^{0}$} & 0.2996 & 0.2798 & $1.982 \mathrm{E}-02$ & 12 & 4 & $7.820 \mathrm{E}-02$ & $1.420 \mathrm{E}+02$ & $7.401 \mathrm{E}+05$ \\
\hline & 155818.710 & 158118.750 & $2.100 \mathrm{E}-02$ & 6 & 4 & $8.286 \mathrm{E}-02$ & $7.102 \mathrm{E}+01$ & $4.403 \mathrm{E}+05$ \\
\hline & 156029.540 & 158118.750 & $1.910 \mathrm{E}-02$ & 4 & 4 & $7.536 \mathrm{E}-02$ & $4.735 \mathrm{E}+01$ & $2.208 \mathrm{E}+05$ \\
\hline & 156148.480 & 158118.750 & $1.800 \mathrm{E}-02$ & 2 & 4 & $7.102 \mathrm{E}-02$ & $2.367 \mathrm{E}+01$ & $9.242 \mathrm{E}+04$ \\
\hline \multirow[t]{8}{*}{$e^{4} P^{6} \rightarrow y^{4} P^{o}$} & 0.2996 & 0.2825 & $1.715 \mathrm{E}-02$ & 12 & 12 & $7.762 \mathrm{E}-02$ & $1.630 \mathrm{E}+02$ & $1.833 \mathrm{E}+05$ \\
\hline & 155818.710 & 158038.600 & $2.030 \mathrm{E}-02$ & 6 & 6 & $6.433 \mathrm{E}-02$ & $5.705 \mathrm{E}+01$ & $2.129 E+05$ \\
\hline & 155818.710 & 157579.680 & $1.610 \mathrm{E}-02$ & 6 & 4 & $2.187 \mathrm{E}-02$ & $2.445 \mathrm{E}+01$ & $6.829 E+04$ \\
\hline & 156029.540 & 158038.600 & $1.840 \mathrm{E}-02$ & 4 & 6 & $3.749 \mathrm{E}-02$ & $2.445 \mathrm{E}+01$ & $6.796 \mathrm{E}+04$ \\
\hline & 156029.540 & 157579.680 & $1.420 \mathrm{E}-02$ & 4 & 4 & $8.572 \mathrm{E}-03$ & $7.244 \mathrm{E}+00$ & $1.388 E+04$ \\
\hline & 156029.540 & 157677.320 & $1.510 \mathrm{E}-02$ & 4 & 2 & $2.849 \mathrm{E}-02$ & $2.264 \mathrm{E}+01$ & $1.043 E+05$ \\
\hline & 156148.480 & 157579.680 & $1.310 \mathrm{E}-02$ & 2 & 4 & $4.942 \mathrm{E}-02$ & $2.264 \mathrm{E}+01$ & $3.406 \mathrm{E}+04$ \\
\hline & 156148.480 & 157677.320 & $1.400 \mathrm{E}-02$ & 2 & 2 & $1.056 \mathrm{E}-02$ & $4.527 \mathrm{E}+\infty 0$ & $1.663 E+04$ \\
\hline \multirow[t]{9}{*}{$e^{4} \mathrm{P}^{\circ} \rightarrow \mathrm{y}^{4} \mathrm{D}^{\circ}$} & 0.2996 & 0.2878 & $1.187 \mathrm{E}-02$ & 12 & 20 & $1.632 \mathrm{E}-05$ & $4.949 \mathrm{E}-02$ & $1.108 \mathrm{E}+01$ \\
\hline & 155818.710 & 157558.770 & $1.590 \mathrm{E}-02$ & 6 & 8 & $1.749 \mathrm{E}-05$ & $1.979 \mathrm{E}-02$ & $2.663 \mathrm{E}+01$ \\
\hline & 155818.710 & 157173.690 & $1.240 \mathrm{E}-02$ & 6 & 6 & $3.068 \mathrm{E}-06$ & $4.454 \mathrm{E}-03$ & $3.789 \mathrm{E}+00$ \\
\hline & 155818.710 & 156939.500 & $1.020 \mathrm{E}-02$ & 6 & 4 & $2.804 \mathrm{E}-07$ & $4.949 \mathrm{E}-04$ & $3.515 \mathrm{E}-01$ \\
\hline & 156029.540 & 157173.690 & $1.050 \mathrm{E}-02$ & 4 & 6 & $9.093 \mathrm{E}-06$ & $1.039 \mathrm{E}-02$ & $5.368 \mathrm{E}+00$ \\
\hline & 156029.540 & 156939.500 & $8.300 E-03$ & 4 & 4 & $3.651 \mathrm{E}-06$ & $5.279 \mathrm{E}-03$ & $2.020 \mathrm{E}+00$ \\
\hline & 156029.540 & 156829.750 & $7.300 E-03$ & 4 & 2 & $5.017 \mathrm{E}-07$ & $8.248 \mathrm{E}-04$ & $4.295 \mathrm{E}-01$ \\
\hline & 156148.480 & 156939.500 & $7.200 \mathrm{E}-03$ & 2 & 4 & $4.949 \mathrm{E}-06$ & $4.124 \mathrm{E}-03$ & $1.030 \mathrm{E}+\infty 0$ \\
\hline & 156148.480 & 156829.750 & $6.200 \mathrm{E}-03$ & 2 & 2 & $4.261 \mathrm{E}-06$ & $4.124 \mathrm{E}-03$ & $1.316 \mathrm{E}+00$ \\
\hline \multirow[t]{9}{*}{$a^{4} D^{\circ} \rightarrow z^{4} P^{0}$} & 0.6797 & 0.5362 & $1.435 \mathrm{E}-01$ & 20 & 12 & $8.286 \mathrm{E}-02$ & $3.464 \mathrm{E}+01$ & $2.284 \mathrm{E}+07$ \\
\hline & 114279.330 & 130134.160 & $1.445 \mathrm{E}-01$ & 8 & 6 & $8.343 \mathrm{E}-02$ & $1.386 \mathrm{E}+01$ & $1.866 \mathrm{E}+07$ \\
\hline & 114231.040 & 130134.160 & $1.450 \mathrm{E}-01$ & 6 & 6 & $2.512 \mathrm{E}-02$ & $3.118 \mathrm{E}+\infty 0$ & $4.242 \mathrm{E}+06$ \\
\hline & 114200.540 & 130134.160 & $1.452 \mathrm{E}-01$ & 4 & 6 & $4.192 \mathrm{E}-03$ & $3.464 \mathrm{E}-01$ & $4.732 \mathrm{E}+05$ \\
\hline & 114231.040 & 129858.180 & $1.425 \mathrm{E}-01$ & 6 & 4 & $5.760 \mathrm{E}-02$ & $7.275 \mathrm{E}+00$ & $1.409 E+07$ \\
\hline & 114200.540 & 129858.180 & $1.427 \mathrm{E}-01$ & 4 & 4 & $4.394 \mathrm{E}-02$ & $3.695 E+\infty 0$ & $7.188 E+06$ \\
\hline & 114162.300 & 129858.180 & $1.431 \mathrm{E}-01$ & 2 & 4 & $1.377 \mathrm{E}-02$ & $5.774 \mathrm{E}-01$ & $1.133 E+06$ \\
\hline & 114200.540 & 129787.830 & $1.420 \mathrm{E}-01$ & 4 & 2 & $3.416 \mathrm{E}-02$ & $2.887 \mathrm{E}+\infty 0$ & $1.107 \mathrm{E}+07$ \\
\hline & 114162.300 & 129787.830 & $1.424 \mathrm{E}-01$ & 2 & 2 & $6.852 \mathrm{E}-02$ & $2.887 \mathrm{E}+\infty$ & $1.116 \mathrm{E}+07$ \\
\hline \multirow[t]{9}{*}{$a^{4} D^{e} \rightarrow y^{4} P^{a}$} & 0.6797 & 0.2825 & $3.972 \mathrm{E}-01$ & 20 & 12 & $5.026 \mathrm{E}-03$ & $7.592 \mathrm{E}-01$ & $1.062 \mathrm{E}+07$ \\
\hline & 114279.330 & 158038.600 & $3.988 \mathrm{E}-01$ & 8 & 6 & $5.046 \mathrm{E}-03$ & $3.037 \mathrm{E}-01$ & $8.595 \mathrm{E}+06$ \\
\hline & 114231.040 & 158038.600 & $3.993 E-01$ & 6 & 6 & $1.516 \mathrm{E}-03$ & $6.833 \mathrm{E}-02$ & $1.941 \mathrm{E}+06$ \\
\hline & 114200.540 & 158038.600 & $3.995 \mathrm{E}-01$ & 4 & 6 & $2.528 \mathrm{E}-04$ & $7.592 \mathrm{E}-03$ & $2.160 \mathrm{E}+05$ \\
\hline & 114231.040 & 157579.680 & $3.951 \mathrm{E}-01$ & 6 & 4 & $3.500 \mathrm{E}-03$ & $1.594 \mathrm{E}-01$ & $6.582 \mathrm{E}+06$ \\
\hline & 114200.540 & 157579.680 & $3.953 \mathrm{E}-01$ & 4 & 4 & $2.668 \mathrm{E}-03$ & $8.098 \mathrm{E}-02$ & $3.348 \mathrm{E}+06$ \\
\hline & 114162.300 & 157579.680 & $3.957 \mathrm{E}-01$ & 2 & 4 & $8.345 \mathrm{E}-04$ & $1.265 \mathrm{E}-02$ & $5.248 \mathrm{E}+05$ \\
\hline & 114200.540 & 157677.320 & $3.962 \mathrm{E}-01$ & 4 & 2 & $2.089 \mathrm{E}-03$ & $6.327 \mathrm{E}-02$ & $5.268 \mathrm{E}+06$ \\
\hline & 114162.300 & 157677.320 & $3.966 \mathrm{E}-01$ & 2 & 2 & $4.182 \mathrm{E}-03$ & $6.327 \mathrm{E}-02$ & $5.284 \mathrm{E}+06$ \\
\hline \multirow[t]{11}{*}{$\mathrm{a}^{4} \mathrm{D}^{\mathrm{e}} \rightarrow \mathrm{z}^{4} \mathrm{D}^{\circ}$} & 0.6797 & 0.5517 & $1.280 \mathrm{E}-01$ & 20 & 20 & $2.308 \mathrm{E}-02$ & $1.081 \mathrm{E}+01$ & $3.039 \mathrm{E}+06$ \\
\hline & 114279.330 & 128599.160 & $1.305 \mathrm{E}-01$ & 8 & 8 & $2.017 \mathrm{E}-02$ & $3.709 \mathrm{E}+\infty 0$ & $2.759 E+06$ \\
\hline & 114279.330 & 128233.200 & $1.271 \mathrm{E}-01$ & 8 & 6 & $3.265 \mathrm{E}-03$ & $6.164 \mathrm{E}-01$ & $5.648 \mathrm{E}+05$ \\
\hline & 114231.040 & 128599.160 & $1.310 \mathrm{E}-01$ & 6 & 8 & $4.486 \mathrm{E}-03$ & $6.164 \mathrm{E}-01$ & $4.638 \mathrm{E}+05$ \\
\hline & 114231.040 & 128233.200 & $1.276 \mathrm{E}-01$ & 6 & 6 & $1.319 \mathrm{E}-02$ & $1.860 \mathrm{E}+00$ & $1.724 \mathrm{E}+06$ \\
\hline & 114231.040 & 127976.340 & $1.253 \mathrm{E}-01$ & 6 & 4 & $5.270 \mathrm{E}-03$ & $7.570 \mathrm{E}-01$ & $9.968 \mathrm{E}+05$ \\
\hline & 114200.540 & 128233.200 & $1.278 \mathrm{E}-01$ & 4 & 6 & $8.062 \mathrm{E}-03$ & $7.570 \mathrm{E}-01$ & $7.051 \mathrm{E}+05$ \\
\hline & 114200.540 & 127976.340 & $1.255 \mathrm{E}-01$ & 4 & 4 & $9.048 \mathrm{E}-03$ & $8.652 \mathrm{E}-01$ & $1.145 E+06$ \\
\hline & 114200.540 & 127825.080 & $1.241 \mathrm{E}-01$ & 4 & 2 & $5.592 \mathrm{E}-03$ & $5.407 \mathrm{E}-01$ & $1.384 \mathrm{E}+06$ \\
\hline & 114162.300 & 127976.340 & $1.259 \mathrm{E}-01$ & 2 & 4 & $1.135 \mathrm{E}-02$ & $5.407 \mathrm{E}-01$ & $7.223 E+05$ \\
\hline & 114162.300 & 127825.080 & $1.245 \mathrm{E}-01$ & 2 & 2 & $1.122 \mathrm{E}-02$ & $5.407 \mathrm{E}-01$ & $1.397 \mathrm{E}+06$ \\
\hline
\end{tabular}


Table IV. Continued

\begin{tabular}{|c|c|c|c|c|c|c|c|c|}
\hline Transition & $\begin{array}{l}E_{i} \\
\mathrm{~cm}^{-1}\end{array}$ & $\begin{array}{l}E_{f} \\
\mathrm{~cm}^{-1}\end{array}$ & $\begin{array}{l}E_{f i} \\
\mathrm{Ry}\end{array}$ & $g_{i}$ & $g_{f}$ & $f_{i f}$ & $S$ & $\begin{array}{l}A_{f_{i}} \\
\mathrm{~s}^{-1} \\
\end{array}$ \\
\hline \multirow[t]{11}{*}{$\mathrm{a}^{4} \mathrm{D}^{\circ} \rightarrow \mathrm{y}^{4} \mathrm{D}^{\circ}$} & 0.6797 & 0.2878 & $3.919 \mathrm{E}-01$ & 20 & 20 & $3.206 \mathrm{E}-04$ & $4.907 \mathrm{E}-02$ & $3.955 \mathrm{E}+05$ \\
\hline & 114279.330 & 157558.770 & $3.944 \mathrm{E}-01$ & 8 & 8 & $2.766 \mathrm{E}-04$ & $1.683 \mathrm{E}-02$ & $3.456 \mathrm{E}+05$ \\
\hline & 114279.330 & 157173.690 & $3.909 \mathrm{E}-01$ & 8 & 6 & $4.556 \mathrm{E}-05$ & $2.797 \mathrm{E}-03$ & $7.456 \mathrm{E}+04$ \\
\hline & 114231.040 & 157558.770 & $3.949 \mathrm{E}-01$ & 6 & 8 & $6.137 \mathrm{E}-05$ & $2.797 \mathrm{E}-03$ & $5.765 E+04$ \\
\hline & 114231.040 & 157173.690 & $3.914 \mathrm{E}-01$ & 6 & 6 & $1.835 \mathrm{E}-04$ & $8.441 \mathrm{E}-03$ & $2.258 \mathrm{E}+05$ \\
\hline & 114231.040 & 156939.500 & $3.892 \mathrm{E}-01$ & 6 & 4 & $7.428 \mathrm{E}-05$ & $3.435 \mathrm{E}-03$ & $1.356 \mathrm{E}+05$ \\
\hline & 114200.540 & 157173.690 & $3.916 \mathrm{E}-01$ & 4 & 6 & $1.121 \mathrm{E}-04$ & $3.435 \mathrm{E}-03$ & $9.205 E+04$ \\
\hline & 114200.540 & 156939.500 & $3.894 \mathrm{E}-01$ & 4 & 4 & $1.274 \mathrm{E}-04$ & $3.926 \mathrm{E}-03$ & $1.552 \mathrm{E}+05$ \\
\hline & 114200.540 & 156829.750 & $3.884 \mathrm{E}-01$ & 4 & 2 & $7.942 \mathrm{E}-05$ & $2.454 \mathrm{E}-03$ & $1.925 \mathrm{E}+05$ \\
\hline & 114162.300 & 156939.500 & $3.898 \mathrm{E}-01$ & 2 & 4 & $1.594 \mathrm{E}-04$ & $2.454 \mathrm{E}-03$ & $9.727 \mathrm{E}+04$ \\
\hline & 114162.300 & 156829.750 & $3.888 \mathrm{E}-01$ & 2 & 2 & $1.590 \mathrm{E}-04$ & $2.454 \mathrm{E}-03$ & $1.931 E+05$ \\
\hline \multirow{9}{*}{$b^{4} D^{e} \rightarrow y^{4} P^{0}$} & 0.3237 & 0.2825 & $4.118 \mathrm{E}-02$ & 20 & 12 & $1.930 \mathrm{E}-01$ & $2.812 \mathrm{E}+02$ & $4.383 E+06$ \\
\hline & 153413.740 & 158038.600 & $4.220 \mathrm{E}-02$ & 8 & 6 & $1.978 \mathrm{E}-01$ & $1.125 \mathrm{E}+02$ & $3.773 E+06$ \\
\hline & 153283.070 & 158038.600 & $4.340 \mathrm{E}-02$ & 6 & 6 & $6.103 \mathrm{E}-02$ & $2.531 \mathrm{E}+01$ & $9.234 \mathrm{E}+05$ \\
\hline & 153201.950 & 158038.600 & $4.410 \mathrm{E}-02$ & 4 & 6 & $1.034 \mathrm{E}-02$ & $2.812 \mathrm{E}+00$ & $1.076 \mathrm{E}+05$ \\
\hline & 153283.070 & 157579.680 & $3.920 \mathrm{E}-02$ & 6 & 4 & $1.286 \mathrm{E}-01$ & $5.906 \mathrm{E}+01$ & $2.381 E+06$ \\
\hline & 153201.950 & 157579.680 & $3.990 \mathrm{E}-02$ & 4 & 4 & $9.975 \mathrm{E}-02$ & $3.000 \mathrm{E}+01$ & $1.276 \mathrm{E}+06$ \\
\hline & 153153.900 & 157579.680 & $4.040 \mathrm{E}-02$ & 2 & 4 & $3.156 \mathrm{E}-02$ & $4.687 \mathrm{E}+00$ & $2.069 \mathrm{E}+05$ \\
\hline & 153201.950 & 157677.320 & $4.080 \mathrm{E}-02$ & 4 & 2 & $7.969 \mathrm{E}-02$ & $2.344 \mathrm{E}+01$ & $2.131 E+06$ \\
\hline & 153153.900 & 157677.320 & $4.130 \mathrm{E}-02$ & 2 & 2 & $1.613 \mathrm{E}-01$ & $2.344 \mathrm{E}+01$ & $2.210 \mathrm{E}+06$ \\
\hline \multirow{11}{*}{$b^{4} D^{6} \rightarrow y^{4} D^{\circ}$} & 0.3237 & 0.2878 & $3.591 \mathrm{E}-02$ & 20 & 20 & $6.435 \mathrm{E}-02$ & $1.075 \mathrm{E}+02$ & $6.664 \mathrm{E}+05$ \\
\hline & 153413.740 & 157558.770 & $3.780 \mathrm{E}-02$ & 8 & 8 & $5.809 \mathrm{E}-02$ & $3.688 \mathrm{E}+01$ & $6.667 \mathrm{E}+05$ \\
\hline & 153413.740 & 157173.690 & $3.430 \mathrm{E}-02$ & 8 & 6 & $8.760 \mathrm{E}-03$ & $6.129 E+00$ & $1.104 \mathrm{E}+05$ \\
\hline & 153283.070 & 157558.770 & $3.900 \mathrm{E}-02$ & 6 & 8 & $1.328 \mathrm{E}-02$ & $6.129 \mathrm{E}+00$ & $1.217 \mathrm{E}+05$ \\
\hline & 153283.070 & 157173.690 & $3.550 \mathrm{E}-02$ & 6 & 6 & $3.648 \mathrm{E}-02$ & $1.850 \mathrm{E}+01$ & $3.692 \mathrm{E}+05$ \\
\hline & 153283.070 & 156939.500 & $3.330 \mathrm{E}-02$ & 6 & 4 & $1.393 \mathrm{E}-02$ & $7.527 \mathrm{E}+00$ & $1.860 \mathrm{E}+05$ \\
\hline & 153201.950 & 157173.690 & $3.620 \mathrm{E}-02$ & 4 & 6 & $2.271 \mathrm{E}-02$ & $7.527 \mathrm{E}+00$ & $1.593 \mathrm{E}+05$ \\
\hline & 153201.950 & 156939.500 & $3.400 \mathrm{E}-02$ & 4 & 4 & $2.437 \mathrm{E}-02$ & $8.603 E+00$ & $2.263 \mathrm{E}+05$ \\
\hline & 153201.950 & 156829.750 & $3.300 \mathrm{E}-02$ & 4 & 2 & $1.479 \mathrm{E}-02$ & $5.377 \mathrm{E}+00$ & $2.587 \mathrm{E}+05$ \\
\hline & 153153.900 & 156939.500 & $3.450 \mathrm{E}-02$ & 2 & 4 & $3.092 \mathrm{E}-02$ & $5.377 \mathrm{E}+00$ & $1.478 \mathrm{E}+05$ \\
\hline & 153153.900 & 156829.750 & $3.350 \mathrm{E}-02$ & 2 & 2 & $3.002 \mathrm{E}-02$ & $5.377 \mathrm{E}+\infty 0$ & $2.706 \mathrm{E}+05$ \\
\hline \multirow[t]{10}{*}{$\mathrm{a}^{4} \mathrm{~F}^{\circ} \rightarrow \mathrm{z}^{4} \mathrm{D}^{\circ}$} & 0.7136 & 0.5517 & $1.620 \mathrm{E}-01$ & 28 & 20 & $1.158 \mathrm{E}-01$ & $6.003 \mathrm{E}+01$ & $3.416 \mathrm{E}+07$ \\
\hline & 110766.560 & 128599.160 & $1.625 \mathrm{E}-01$ & 10 & 8 & $1.161 \mathrm{E}-01$ & $2.144 \mathrm{E}+01$ & $3.079 E+07$ \\
\hline & 110508.710 & 128599.160 & $1.649 \mathrm{E}-01$ & 8 & 8 & $1.679 \mathrm{E}-02$ & $2.444 E+00$ & $3.668 \mathrm{E}+06$ \\
\hline & 110313.400 & 128599.160 & $1.667 \mathrm{E}-01$ & 6 & 8 & $1.152 \mathrm{E}-03$ & $1.244 \mathrm{E}-01$ & $1.928 \mathrm{E}+05$ \\
\hline & 110508.710 & 128233.200 & $1.615 \mathrm{E}-01$ & 8 & 6 & $9.897 \mathrm{E}-02$ & $1.471 \mathrm{E}+01$ & $2.765 \mathrm{E}+07$ \\
\hline & 110313.400 & 128233.200 & $1.633 \mathrm{E}-01$ & 6 & 6 & $2.840 \mathrm{E}-02$ & $3.130 \mathrm{E}+00$ & $6.083 E+06$ \\
\hline & 110177.020 & 128233.200 & $1.645 \mathrm{E}-01$ & 4 & 6 & $2.351 \mathrm{E}-03$ & $1.715 \mathrm{E}-01$ & $3.407 \mathrm{E}+05$ \\
\hline & 110313.400 & 127976.340 & $1.610 \mathrm{E}-01$ & 6 & 4 & $8.630 \mathrm{E}-02$ & $9.648 \mathrm{E}+00$ & $2.695 \mathrm{E}+07$ \\
\hline & 110177.020 & 127976.340 & $1.622 \mathrm{E}-01$ & 4 & 4 & $3.246 \mathrm{E}-02$ & $2.401 E+00$ & $6.859 \mathrm{E}+06$ \\
\hline & 110177.020 & 127825.080 & $1.608 \mathrm{E}-01$ & 4 & 2 & $8.045 E-02$ & $6.003 \mathrm{E}+00$ & $3.341 \mathrm{E}+07$ \\
\hline \multirow[t]{10}{*}{$a^{4} F^{e} \rightarrow y^{4} D^{\circ}$} & 0.7136 & 0.2878 & $4.259 \mathrm{E}-01$ & 28 & 20 & $6.962 \mathrm{E}-03$ & $1.373 \mathrm{E}+00$ & $1.420 \mathrm{E}+07$ \\
\hline & 110766.560 & 157558.770 & $4.264 \mathrm{E}-01$ & 10 & 8 & $6.971 \mathrm{E}-03$ & $4.904 \mathrm{E}-01$ & $1.273 E+07$ \\
\hline & 110508.710 & 157558.770 & $4.288 \mathrm{E}-01$ & 8 & 8 & $9.989 \mathrm{E}-04$ & $5.591 \mathrm{E}-02$ & $1.475 \mathrm{E}+06$ \\
\hline & 110313.400 & 157558.770 & $4.306 \mathrm{E}-01$ & 6 & 8 & $6.805 \mathrm{E}-05$ & $2.845 \mathrm{E}-03$ & $7.601 \mathrm{E}+04$ \\
\hline & 110508.710 & 157173.690 & $4.253 \mathrm{E}-01$ & 8 & 6 & $5.962 \mathrm{E}-03$ & $3.364 \mathrm{E}-01$ & $1.155 \mathrm{E}+07$ \\
\hline & 110313.400 & 157173.690 & $4.271 \mathrm{E}-01$ & 6 & 6 & $1.699 \mathrm{E}-03$ & $7.160 \mathrm{E}-02$ & $2.489 \mathrm{E}+06$ \\
\hline & 110177.020 & 157173.690 & $4.283 \mathrm{E}-01$ & 4 & 6 & $1.400 \mathrm{E}-04$ & $3.923 \mathrm{E}-03$ & $1.376 \mathrm{E}+05$ \\
\hline & 110313.400 & 156939.500 & $4.249 \mathrm{E}-01$ & 6 & 4 & $5.210 \mathrm{E}-03$ & $2.207 \mathrm{E}-01$ & $1.133 \mathrm{E}+07$ \\
\hline & 110177.020 & 156939.500 & $4.261 \mathrm{E}-01$ & 4 & 4 & $1.950 \mathrm{E}-03$ & $5.493 \mathrm{E}-02$ & $2.844 \mathrm{E}+06$ \\
\hline & 110177.020 & 156829.750 & $4.251 \mathrm{E}-01$ & 4 & 2 & $4.865 \mathrm{E}-03$ & $1.373 E-01$ & $1.412 E+07$ \\
\hline \multirow[t]{10}{*}{$b^{4} F^{e} \rightarrow y^{4} D^{\circ}$} & 0.3326 & 0.2878 & $4.488 \mathrm{E}-02$ & 28 & 20 & $2.580 \mathrm{E}-01$ & $4.829 \mathrm{E}+02$ & $5.844 \mathrm{E}+06$ \\
\hline & 152615.460 & 157558.770 & $4.510 \mathrm{E}-02$ & 10 & 8 & $2.593 \mathrm{E}-01$ & $1.725 \mathrm{E}+02$ & $5.295 \mathrm{E}+06$ \\
\hline & 152305.000 & 157558.770 & $4.790 \mathrm{E}-02$ & 8 & 8 & $3.924 \mathrm{E}-02$ & $1.966 \mathrm{E}+01$ & $7.232 \mathrm{E}+05$ \\
\hline & 152094.640 & 157558.770 & $4.980 \mathrm{E}-02$ & 6 & 8 & $2.768 \mathrm{E}-03$ & $1.000 \mathrm{E}+00$ & $4.135 \mathrm{E}+04$ \\
\hline & 152305.000 & 157173.690 & $4.440 \mathrm{E}-02$ & 8 & 6 & $2.189 \mathrm{E}-01$ & $1.183 E+02$ & $4.621 E+06$ \\
\hline & 152094.640 & 157173.690 & $4.630 \mathrm{E}-02$ & 6 & 6 & $6.477 \mathrm{E}-02$ & $2.518 \mathrm{E}+01$ & $1.115 \mathrm{E}+06$ \\
\hline & 151959.690 & 157173.690 & $4.750 \mathrm{E}-02$ & 4 & 6 & $5.462 \mathrm{E}-03$ & $1.380 \mathrm{E}+\infty 0$ & $6.599 \mathrm{E}+04$ \\
\hline & 152094.640 & 156939.500 & $4.410 \mathrm{E}-02$ & 6 & 4 & $1.902 \mathrm{E}-01$ & $7.761 \mathrm{E}+01$ & $4.456 \mathrm{E}+06$ \\
\hline & 151959.690 & 156939.500 & $4.530 \mathrm{E}-02$ & 4 & 4 & $7.292 \mathrm{E}-02$ & $1.932 \mathrm{E}+01$ & $1.202 \mathrm{E}+06$ \\
\hline & 151959.690 & 156829.750 & $4.430 \mathrm{E}-02$ & 4 & 2 & $1.783 \mathrm{E}-01$ & $4.829 \mathrm{E}+01$ & $5.621 E+06$ \\
\hline
\end{tabular}


Table IV. Continued

\begin{tabular}{|c|c|c|c|c|c|c|c|c|}
\hline Transition & $\begin{array}{l}E_{i} \\
\mathrm{~cm}^{-1} \\
\end{array}$ & $\begin{array}{l}E_{f} \\
\mathrm{~cm}^{-1} \\
\end{array}$ & $\begin{array}{l}E_{f i} \\
\mathrm{Ry}\end{array}$ & $g_{i}$ & $g_{f}$ & $f_{i f}$ & $s$ & $\begin{array}{l}A_{f i} \\
\mathrm{~s}^{-1} \\
\end{array}$ \\
\hline \multirow{8}{*}{$z^{4} P^{o} \rightarrow c^{4} P^{e}$} & 0.5362 & 0.5294 & $6.814 \mathrm{E}-03$ & 12 & 12 & $6.164 \mathrm{E}-03$ & $3.257 \mathrm{E}+01$ & $2.299 \mathrm{E}+03$ \\
\hline & 130134.160 & 130602.210 & $4.200 \mathrm{E}-03$ & 6 & 6 & $2.660 \mathrm{E}-03$ & $1.140 \mathrm{E}+01$ & $3.768 \mathrm{E}+02$ \\
\hline & 130134.160 & 130818.850 & $6.200 \mathrm{E}-03$ & 6 & 4 & $1.683 \mathrm{E}-03$ & $4.885 \mathrm{E}+00$ & $7.793 \mathrm{E}+02$ \\
\hline & 129858.180 & 130602.210 & $6.700 \mathrm{E}-03$ & 4 & 6 & $2.727 \mathrm{E}-03$ & $4.885 \mathrm{E}+00$ & $6.556 \mathrm{E}+02$ \\
\hline & 129858.180 & 130818.850 & $8.700 \mathrm{E}-03$ & 4 & 4 & $1.049 \mathrm{E}-03$ & $1.447 \mathrm{E}+00$ & $6.380 \mathrm{E}+02$ \\
\hline & 129858.180 & 130948.940 & $9.900 \mathrm{E}-03$ & 4 & 2 & $3.732 \mathrm{E}-03$ & $4.523 \mathrm{E}+\infty 0$ & $5.875 \mathrm{E}+03$ \\
\hline & 129787.830 & 130818.850 & $9.400 \mathrm{E}-03$ & 2 & 4 & $7.086 \mathrm{E}-03$ & $4.523 \mathrm{E}+\infty$ & $2.515 \mathrm{E}+03$ \\
\hline & 129787.830 & 130948.940 & $1.060 \mathrm{E}-02$ & 2 & 2 & $1.598 \mathrm{E}-03$ & $9.046 \mathrm{E} 01$ & $1.442 \mathrm{E}+03$ \\
\hline \multirow{8}{*}{$\mathrm{z}^{4} \mathrm{P}^{0} \rightarrow \mathrm{d}^{4} \mathrm{P}^{e}$} & 0.5362 & 0.3473 & $1.889 \mathrm{E}-01$ & 12 & 12 & $1.962 \mathrm{E}-01$ & $3.738 \mathrm{E}+01$ & $5.625 \mathrm{E}+07$ \\
\hline & 130134.160 & 150996.410 & $1.901 \mathrm{E}-01$ & 6 & 6 & $1.382 \mathrm{E}-01$ & $1.308 \mathrm{E}+01$ & $4.011 \mathrm{E}+07$ \\
\hline & 130134.160 & 150531.310 & $1.858 \mathrm{E}-01$ & 6 & 4 & $5.787 \mathrm{E}-02$ & $5.607 \mathrm{E}+00$ & $2.407 \mathrm{E}+07$ \\
\hline & 129858.180 & 150996.410 & $1.926 \mathrm{E}-01$ & 4 & 6 & $8.999 \mathrm{E}-02$ & $5.607 \mathrm{E}+00$ & $1.788 \mathrm{E}+07$ \\
\hline & 129858.180 & 150531.310 & $1.883 \mathrm{E}-01$ & 4 & 4 & $2.607 \mathrm{E}-02$ & $1.661 \mathrm{E}+00$ & $7.424 \mathrm{E}+06$ \\
\hline & 129858.180 & 150258.510 & $1.859 \mathrm{E}-01$ & 4 & 2 & $8.042 \mathrm{E}-02$ & $5.191 \mathrm{E}+00$ & $4.465 \mathrm{E}+07$ \\
\hline & 129787.830 & 150531.310 & $1.890 \mathrm{E}-01$ & 2 & 4 & $1.635 \mathrm{E}-01$ & $5.191 \mathrm{E}+\infty 0$ & $2.346 \mathrm{E}+07$ \\
\hline & 129787.830 & 150258.510 & $1.866 \mathrm{E}-01$ & 2 & 2 & $3.229 \mathrm{E}-02$ & $1.038 \mathrm{E}+00$ & $9.031 \mathrm{E}+06$ \\
\hline \multirow{8}{*}{$z^{4} P^{0} \rightarrow e^{4} P^{e}$} & 0.5362 & 0.2996 & $2.366 \mathrm{E}-01$ & 12 & 12 & $2.016 \mathrm{E}-01$ & $3.067 \mathrm{E}+01$ & $9.060 \mathrm{E}+07$ \\
\hline & 130134.160 & 155818.710 & $2.340 \mathrm{E}-01$ & 6 & 6 & $1.396 \mathrm{E}-01$ & $1.074 \mathrm{E}+01$ & $6.138 \mathrm{E}+07$ \\
\hline & 130134.160 & 156029.540 & $2.359 \mathrm{E}-01$ & 6 & 4 & $6.030 \mathrm{E}-02$ & $4.601 E+00$ & $4.043 \mathrm{E}+07$ \\
\hline & 129858.180 & 155818.710 & $2.365 \mathrm{E}-01$ & 4 & 6 & $9.068 \mathrm{E}-02$ & $4.601 E+00$ & $2.716 \mathrm{E}+07$ \\
\hline & 129858.180 & 156029.540 & $2.384 \mathrm{E}-01$ & 4 & 4 & $2.708 \mathrm{E}-02$ & $1.363 \mathrm{E}+00$ & $1.236 \mathrm{E}+07$ \\
\hline & 129858.180 & 156148.480 & $2.395 \mathrm{E}-01$ & 4 & 2 & $8.503 \mathrm{E}-02$ & $4.260 \mathrm{E}+00$ & $7.835 \mathrm{E}+07$ \\
\hline & 129787.830 & 156029.540 & $2.391 \mathrm{E}-01$ & 2 & 4 & $1.698 \mathrm{E}-01$ & $4.260 \mathrm{E}+00$ & $3.898 \mathrm{E}+07$ \\
\hline & 129787.830 & 156148.480 & $2.402 \mathrm{E}-01$ & 2 & 2 & $3.411 \mathrm{E}-02$ & $8.521 \mathrm{E}-01$ & $1.581 \mathrm{E}+07$ \\
\hline \multirow[t]{8}{*}{$\mathrm{z}^{4} \mathrm{P}^{\circ} \rightarrow \mathrm{f}^{4} \mathrm{P}^{e}$} & 0.5362 & 0.2063 & $3.299 \mathrm{E}-01$ & 12 & 12 & $2.549 \mathrm{E}-02$ & $2.781 \mathrm{E}+00$ & $2.229 \mathrm{E}+07$ \\
\hline & 130134.160 & 166479.820 & $3.312 \mathrm{E}-01$ & 6 & 6 & $1.791 \mathrm{E}-02$ & $9.735 \mathrm{E}-01$ & $1.578 \mathrm{E}+07$ \\
\hline & 130134.160 & 165991.350 & $3.267 \mathrm{E}-01$ & 6 & 4 & $7.572 \mathrm{E}-03$ & $4.172 \mathrm{E}-01$ & $9.737 E+06$ \\
\hline & 129858.180 & 166479.820 & $3.337 \mathrm{E}-01$ & 4 & 6 & $1.160 \mathrm{E}-02$ & $4.172 \mathrm{E}-01$ & $6.918 E+06$ \\
\hline & 129858.180 & 165991.350 & $3.292 \mathrm{E}-01$ & 4 & 4 & $3.391 \mathrm{E}-03$ & $1.236 \mathrm{E}-01$ & $2.952 E+06$ \\
\hline & 129858.180 & 165719.230 & $3.267 \mathrm{E}-01$ & 4 & 2 & $1.052 \mathrm{E}-02$ & $3.863 \mathrm{E}-01$ & $1.803 E+07$ \\
\hline & 129787.830 & 165991.350 & $3.299 \mathrm{E}-01$ & 2 & 4 & $2.124 \mathrm{E}-02$ & $3.863 E-01$ & $9.284 \mathrm{E}+06$ \\
\hline & 129787.830 & 165719.230 & $3.274 \mathrm{E}-01$ & 2 & 2 & $4.216 \mathrm{E}-03$ & $7.726 \mathrm{E}-02$ & $3.630 \mathrm{E}+06$ \\
\hline \multirow[t]{8}{*}{$z^{4} P^{\circ} \rightarrow g{ }^{4} P^{e}$} & 0.5362 & 0.1868 & $3.494 \mathrm{E}-01$ & 12 & 12 & $3.250 \mathrm{E}-02$ & $3.349 \mathrm{E}+00$ & $3.186 \mathrm{E}+07$ \\
\hline & 130134.160 & 168217.100 & $3.470 \mathrm{E}-01$ & 6 & 6 & $2.259 \mathrm{E}-02$ & $1.172 \mathrm{E}+00$ & $2.185 \mathrm{E}+07$ \\
\hline & 130134.160 & 168397.890 & $3.487 \mathrm{E}-01$ & 6 & 4 & $9.731 \mathrm{E}-03$ & $5.023 \mathrm{E}-01$ & $1.426 \mathrm{E}+07$ \\
\hline & 129858.180 & 168217.100 & $3.495 \mathrm{E}-01$ & 4 & 6 & $1.463 \mathrm{E}-02$ & $5.023 \mathrm{E}-01$ & $9.569 E+06$ \\
\hline & 129858.180 & 168397.890 & $3.512 \mathrm{E}-01$ & 4 & 4 & $4.356 \mathrm{E}-03$ & $1.488 \mathrm{E}-01$ & $4.315 E+06$ \\
\hline & 129858.180 & 168490.430 & $3.520 \mathrm{E}-01$ & 4 & 2 & $1.364 \mathrm{E}-02$ & $4.651 \mathrm{E}-01$ & $2.715 E+07$ \\
\hline & 129787.830 & 168397.890 & $3.519 \mathrm{E}-01$ & 2 & 4 & $2.728 \mathrm{E}-02$ & $4.651 \mathrm{E}-01$ & $1.357 \mathrm{E}+07$ \\
\hline & 129787.830 & 168490.430 & $3.527 \mathrm{E}-01$ & 2 & 2 & $5.468 \mathrm{E}-03$ & $9.302 \mathrm{E}-02$ & $5.463 E+06$ \\
\hline \multirow[t]{8}{*}{$z^{4} P^{0} \rightarrow h^{4} P^{e}$} & 0.5362 & 0.1369 & $3.993 \mathrm{E}-01$ & 12 & 12 & $9.135 \mathrm{E}-03$ & $8.237 \mathrm{E}-01$ & $1.170 \mathrm{E}+07$ \\
\hline & 130134.160 & 174098.100 & $4.006 \mathrm{E}-01$ & 6 & 6 & $6.416 \mathrm{E}-03$ & $2.883 \mathrm{E}-01$ & $8.270 \mathrm{E}+06$ \\
\hline & 130134.160 & 173595.770 & $3.960 \mathrm{E}-01$ & 6 & 4 & $2.718 \mathrm{E}-03$ & $1.236 \mathrm{E}-01$ & $5.136 \mathrm{E}+06$ \\
\hline & 129858.180 & 174098.100 & $4.031 \mathrm{E}-01$ & 4 & 6 & $4.150 \mathrm{E}-03$ & $1.236 \mathrm{E}-01$ & $3.611 \mathrm{E}+06$ \\
\hline & 129858.180 & 173595.770 & $3.985 \mathrm{E}-01$ & 4 & 4 & $1.216 \mathrm{E}-03$ & $3.661 \mathrm{E}-02$ & $1.551 \mathrm{E}+06$ \\
\hline & 129858.180 & 173316.830 & $3.960 \mathrm{E}-01$ & 4 & 2 & $3.775 \mathrm{E}-03$ & $1.144 \mathrm{E}-01$ & $9.510 \mathrm{E}+06$ \\
\hline & 129787.830 & 173595.770 & $3.992 \mathrm{E}-01$ & 2 & 4 & $7.611 \mathrm{E}-03$ & $1.144 \mathrm{E}-01$ & $4.871 \mathrm{E}+06$ \\
\hline & 129787.830 & 173316.830 & $3.967 \mathrm{E}-01$ & 2 & 2 & $1.513 \mathrm{E}-03$ & $2.288 \mathrm{E}-02$ & $1.912 \mathrm{E}+06$ \\
\hline \multirow[t]{8}{*}{$\mathrm{z}^{4} \mathrm{P}^{\mathrm{o}} \rightarrow \mathrm{i}^{4} \mathrm{P}^{\mathrm{e}}$} & 0.5362 & 0.1263 & $4.099 \mathrm{E}-01$ & 12 & 12 & $1.050 \mathrm{E}-02$ & $9.221 \mathrm{E}-01$ & $1.417 \mathrm{E}+07$ \\
\hline & 130134.160 & 174892.750 & $4.078 \mathrm{E}-01$ & 6 & 6 & $7.312 \mathrm{E}-03$ & $3.227 \mathrm{E}-01$ & $9.767 \mathrm{E}+06$ \\
\hline & 130134.160 & 175019.780 & $4.090 \mathrm{E}-01$ & 6 & 4 & $3.143 \mathrm{E}-03$ & $1.383 \mathrm{E}-01$ & $6.334 \mathrm{E}+06$ \\
\hline & 129858.180 & 174892.750 & $4.103 E-01$ & 4 & 6 & $4.729 \mathrm{E}-03$ & $1.383 \mathrm{E}-01$ & $4.263 E+06$ \\
\hline & 129858.180 & 175019.780 & $4.115 \mathrm{E}-01$ & 4 & 4 & $1.405 \mathrm{E}-03$ & $4.098 \mathrm{E}-02$ & $1.911 \mathrm{E}+06$ \\
\hline & 129858.180 & 175083.510 & $4.121 \mathrm{E}-01$ & 4 & 2 & $4.398 \mathrm{E}-03$ & $1.281 \mathrm{E}-01$ & $1.200 \mathrm{E}+07$ \\
\hline & 129787.830 & 175019.780 & $4.122 \mathrm{E}-01$ & 2 & 4 & $8.798 \mathrm{E}-03$ & $1.281 \mathrm{E}-01$ & $6.004 \mathrm{E}+06$ \\
\hline & 129787.830 & 175083.510 & $4.128 \mathrm{E}-01$ & 2 & 2 & $1.762 \mathrm{E}-03$ & $2.561 \mathrm{E}-02$ & $2.412 \mathrm{E}+06$ \\
\hline
\end{tabular}


Table IV. Continued

\begin{tabular}{|c|c|c|c|c|c|c|c|c|}
\hline Transition & $\begin{array}{l}E_{i} \\
\mathrm{~cm}^{-1}\end{array}$ & $\begin{array}{l}E_{f} \\
\mathrm{~cm}^{-1}\end{array}$ & $\begin{array}{l}E_{f l} \\
\text { Ry }\end{array}$ & $g_{i}$ & $g_{f}$ & $f_{i f}$ & $s$ & $\begin{array}{l}A_{f i} \\
\mathrm{~s}^{-1} \\
\end{array}$ \\
\hline \multirow[t]{8}{*}{$\mathrm{z}^{4} \mathrm{P}^{0} \rightarrow \mathrm{j}^{4} \mathrm{P}^{\mathrm{e}}$} & 0.5362 & 0.0905 & $4.457 \mathrm{E}-01$ & 12 & 12 & $4.550 \mathrm{E}-03$ & $3.675 \mathrm{E}-01$ & $7.259 E+06$ \\
\hline & 130134.160 & 178848.960 & $4.439 \mathrm{E}-01$ & 6 & 6 & $3.172 \mathrm{E}-03$ & $1.286 \mathrm{E}-01$ & $5.021 \mathrm{E}+06$ \\
\hline & 130134.160 & 178915.200 & $4.445 \mathrm{E}-01$ & 6 & 4 & $1.361 \mathrm{E}-03$ & $5.513 \mathrm{E}-02$ & $3.241 E+06$ \\
\hline & 129858.180 & 178848.960 & $4.464 \mathrm{E}-01$ & 4 & 6 & $2.051 \mathrm{E}-03$ & $5.513 \mathrm{E}-02$ & $2.188 \mathrm{E}+06$ \\
\hline & 129858.180 & 178915.200 & $4.470 \mathrm{E}-01$ & 4 & 4 & $6.085 \mathrm{E}-04$ & $1.633 \mathrm{E}-02$ & $9.765 \mathrm{E}+05$ \\
\hline & 129858.180 & 178969.320 & $4.475 \mathrm{E}-01$ & 4 & 2 & $1.904 \mathrm{E}-03$ & $5.105 \mathrm{E}-02$ & $6.124 E+06$ \\
\hline & 129787.830 & 178915.200 & $4.477 \mathrm{E}-01$ & 2 & 4 & $3.809 \mathrm{E}-03$ & $5.105 \mathrm{E}-02$ & $3.066 \mathrm{E}+06$ \\
\hline & 129787.830 & 178969.320 & $4.482 \mathrm{E}-01$ & 2 & 2 & $7.626 \mathrm{E}-04$ & $1.021 \mathrm{E}-02$ & $1.231 \mathrm{E}+06$ \\
\hline \multirow[t]{9}{*}{$z^{4} P^{0} \rightarrow b^{4} D^{e}$} & 0.5362 & 0.3237 & $2.125 \mathrm{E}-01$ & 12 & 20 & $7.842 \mathrm{E}-01$ & $1.328 \mathrm{E}+02$ & $1.707 \mathrm{E}+08$ \\
\hline & 130134.160 & 153413.740 & $2.121 \mathrm{E}-01$ & 6 & 8 & $6.261 \mathrm{E}-01$ & $5.314 \mathrm{E}+01$ & $1.697 \mathrm{E}+08$ \\
\hline & 130134.160 & 153283.070 & $2.109 \mathrm{E}-01$ & 6 & 6 & $1.401 \mathrm{E}-01$ & $1.196 \mathrm{E}+01$ & $5.004 E+07$ \\
\hline & 130134.160 & 153201.950 & $2.102 \mathrm{E}-01$ & 6 & 4 & $1.551 \mathrm{E}-02$ & $1.328 \mathrm{E}+00$ & $8.258 \mathrm{E}+06$ \\
\hline & 129858.180 & 153283.070 & $2.134 \mathrm{E}-01$ & 4 & 6 & $4.961 \mathrm{E}-01$ & $2.790 \mathrm{E}+01$ & $1.210 \mathrm{E}+08$ \\
\hline & 129858.180 & 153201.950 & $2.127 \mathrm{E}-01$ & 4 & 4 & $2.512 \mathrm{E}-01$ & $1.417 \mathrm{E}+01$ & $9.127 E+07$ \\
\hline & 129858.180 & 153153.900 & $2.122 \mathrm{E}-01$ & 4 & 2 & $3.915 \mathrm{E}-02$ & $2.214 \mathrm{E}+00$ & $2.832 \mathrm{E}+07$ \\
\hline & 129787.830 & 153201.950 & $2.134 \mathrm{E}-01$ & 2 & 4 & $3.937 \mathrm{E}-01$ & $1.107 \mathrm{E}+01$ & $7.201 \mathrm{E}+07$ \\
\hline & 129787.830 & 153153.900 & $2.129 \mathrm{E}-01$ & 2 & 2 & $3.928 \mathrm{E}-01$ & $1.107 \mathrm{E}+01$ & $1.430 \mathrm{E}+08$ \\
\hline \multirow[t]{9}{*}{$\mathrm{z}^{4} \mathrm{P}^{0} \rightarrow \mathrm{c}^{4} \mathrm{D}^{\mathrm{a}}$} & 0.5362 & 0.1950 & $3.412 \mathrm{E}-01$ & 12 & 20 & $6.765 \mathrm{E}-02$ & $7.138 \mathrm{E}+00$ & $3.795 \mathrm{E}+07$ \\
\hline & 130134.160 & 167575.230 & $3.412 \mathrm{E}-01$ & 6 & 8 & $5.412 \mathrm{E}-02$ & $2.855 \mathrm{E}+\infty 0$ & $3.796 \mathrm{E}+07$ \\
\hline & 130134.160 & 167364.980 & $3.392 \mathrm{E}-01$ & 6 & 6 & $1.211 \mathrm{E}-02$ & $6.424 \mathrm{E}-01$ & $1.119 \mathrm{E}+07$ \\
\hline & 130134.160 & 167291.840 & $3.386 \mathrm{E}-01$ & 6 & 4 & $1.343 \mathrm{E}-03$ & $7.138 \mathrm{E}-02$ & $1.855 E+06$ \\
\hline & 129858.180 & 167364.980 & $3.417 \mathrm{E}-01$ & 4 & 6 & $4.268 \mathrm{E}-02$ & $1.499 \mathrm{E}+00$ & $2.669 \mathrm{E}+07$ \\
\hline & 129858.180 & 167291.840 & $3.411 \mathrm{E}-01$ & 4 & 4 & $2.164 \mathrm{E}-02$ & $7.614 \mathrm{E}-01$ & $2.023 \mathrm{E}+07$ \\
\hline & 129858.180 & 167254.840 & $3.407 \mathrm{E}-01$ & 4 & 2 & $3.378 \mathrm{E}-03$ & $1.190 \mathrm{E}-01$ & $6.299 E+06$ \\
\hline & 129787.830 & 167291.840 & $3.418 \mathrm{E}-01$ & 2 & 4 & $3.389 \mathrm{E}-02$ & $5.949 \mathrm{E}-01$ & $1.590 \mathrm{E}+07$ \\
\hline & 129787.830 & 167254.840 & $3.414 \mathrm{E}-01$ & 2 & 2 & $3.385 \mathrm{E}-02$ & $5.949 \mathrm{E}-01$ & $3.169 E+07$ \\
\hline \multirow{9}{*}{$\begin{array}{c}\mathrm{z}^{4} \mathrm{P}^{\circ} \rightarrow \mathrm{d}^{4} \mathrm{D}^{\circ} \\
*\end{array}$} & 0.5362 & 0.1319 & $4.043 \mathrm{E}-01$ & 12 & 20 & $1.867 \mathrm{E}-02$ & $1.663 \mathrm{E}+00$ & $1.471 \mathrm{E}+07$ \\
\hline & & & & 6 & 8 & $1.494 \mathrm{E}-02$ & $6.652 \mathrm{E}-01$ & $1.471 E+07$ \\
\hline & 130134.160 & 174360.920 & $4.030 \mathrm{E}-01$ & 6 & 6 & $3.351 \mathrm{E}-03$ & $1.497 \mathrm{E}-01$ & $4.371 \mathrm{E}+06$ \\
\hline & 130134.160 & 174329.310 & $4.027 \mathrm{E}-01$ & 6 & 4 & $3.720 \mathrm{E}-04$ & $1.663 \mathrm{E}-02$ & $7.269 \mathrm{E}+05$ \\
\hline & 129858.180 & 174360.920 & $4.055 \mathrm{E}-01$ & 4 & 6 & $1.180 \mathrm{E}-02$ & $3.492 \mathrm{E}-01$ & $1.039 \mathrm{E}+07$ \\
\hline & 129858.180 & 174329.310 & $4.052 \mathrm{E}-01$ & 4 & 4 & $5.989 \mathrm{E}-03$ & $1.774 \mathrm{E}-01$ & $7.899 \mathrm{E}+06$ \\
\hline & & & & 4 & 2 & $9.337 \mathrm{E}-0.4$ & $2.772 \mathrm{E}-02$ & $2.451 E+06$ \\
\hline & 129787.830 & 174329.310 & $4.059 \mathrm{E}-01$ & 2 & 4 & $9.375 \mathrm{E}-03$ & $1.386 \mathrm{E}-01$ & $6.203 E+06$ \\
\hline & & & & 2 & 2 & $9.337 \mathrm{E}-03$ & $1.386 \mathrm{E}-01$ & $1.226 \mathrm{E}+07$ \\
\hline \multirow{9}{*}{$\begin{array}{c}\mathrm{z}^{4} \mathrm{P}^{0} \rightarrow \mathrm{e}^{4} \mathrm{D}^{e} \\
*\end{array}$} & 0.5362 & 0.0955 & $4.407 \mathrm{E}-01$ & 12 & 20 & $7.818 \mathrm{E}-03$ & $6.387 \mathrm{E}-01$ & $7.317 \mathrm{E}+06$ \\
\hline & & & & 6 & 8 & $6.254 \mathrm{E}-03$ & $2.555 \mathrm{E}-01$ & $7.317 \mathrm{E}+06$ \\
\hline & 130134.160 & 178344.360 & $4.393 \mathrm{E}-01$ & 6 & 6 & $1.403 E-03$ & $5.748 \mathrm{E}-02$ & $2.175 \mathrm{E}+06$ \\
\hline & & & & 6 & 4 & $1.564 \mathrm{E}-04$ & $6.387 \mathrm{E}-03$ & $3.659 \mathrm{E}+05$ \\
\hline & 129858.180 & 178344.360 & $4.418 \mathrm{E}-01$ & 4 & 6 & $4.938 \mathrm{E}-03$ & $1.341 \mathrm{E}-01$ & $5.161 E+06$ \\
\hline & & & & 4 & 4 & $2.502 \mathrm{E}-03$ & $6.812 \mathrm{E}-02$ & $3.903 \mathrm{E}+06$ \\
\hline & & & & 4 & 2 & $3.909 E-04$ & $1.064 \mathrm{E}-02$ & $1.220 \mathrm{E}+06$ \\
\hline & & & & 2 & 4 & $3.909 \mathrm{E}-03$ & $5.322 \mathrm{E}-02$ & $3.049 E+06$ \\
\hline & & & & 2 & 2 & $3.909 \mathrm{E}-03$ & $5.322 \mathrm{E}-02$ & $6.098 \mathrm{E}+06$ \\
\hline \multirow[t]{8}{*}{$y^{4} P^{o} \rightarrow f^{4} P^{e}$} & 0.2825 & 0.2063 & $7.623 \mathrm{E}-02$ & 12 & 12 & $3.009 \mathrm{E}-01$ & $1.421 \mathrm{E}+02$ & $1.404 \mathrm{E}+\mathbf{0 7}$ \\
\hline & 158038.600 & 166479.820 & $7.690 \mathrm{E}-02$ & 6 & 6 & $2.125 \mathrm{E}-01$ & $4.973 E+01$ & $1.009 \mathrm{E}+07$ \\
\hline & 158038.600 & 165991.350 & $7.240 \mathrm{E}-02$ & 6 & 4 & $8.573 E-02$ & $2.131 E+01$ & $5.414 \mathrm{E}+06$ \\
\hline & 157579.680 & 166479.820 & $8.110 \mathrm{E}-02$ & 4 & 6 & $1.440 \mathrm{E}-01$ & $2.131 \mathrm{E}+01$ & $5.073 \mathrm{E}+06$ \\
\hline & 157579.680 & 165991.350 & $7.660 \mathrm{E}-02$ & 4 & 4 & $4.031 \mathrm{E}-02$ & $6.315 \mathrm{E}+00$ & $1.900 \mathrm{E}+06$ \\
\hline & 157579.680 & 165719.230 & $7.410 \mathrm{E}-02$ & 4 & 2 & $1.219 \mathrm{E}-01$ & $1.973 \mathrm{E}+01$ & $1.075 \mathrm{E}+07$ \\
\hline & 157677.320 & 165991.350 & $7.570 \mathrm{E}-02$ & 2 & 4 & $2.490 \mathrm{E}-01$ & $1.973 \mathrm{E}+01$ & $5.730 \mathrm{E}+06$ \\
\hline & 157677.320 & 165719.230 & $7.320 \mathrm{E}-02$ & 2 & 2 & $4.815 \mathrm{E}-02$ & $3.947 \mathrm{E}+00$ & $2.072 \mathrm{E}+06$ \\
\hline \multirow[t]{8}{*}{$y^{4} P^{o} \rightarrow g^{4} P^{e}$} & 0.2825 & 0.1868 & $9.566 \mathrm{E}-02$ & 12 & 12 & $2.546 \mathrm{E}-01$ & $9.581 \mathrm{E}+01$ & $1.871 \mathrm{E}+07$ \\
\hline & 158038.600 & 168217.100 & $9.270 \mathrm{E}-02$ & 6 & 6 & $1.727 \mathrm{E}-01$ & $3.353 \mathrm{E}+01$ & $1.192 \mathrm{E}+07$ \\
\hline & 158038.600 & 168397.890 & $9.440 \mathrm{E}-02$ & 6 & 4 & $7.537 \mathrm{E}-02$ & $1.437 \mathrm{E}+01$ & $8.093 E+06$ \\
\hline & 157579.680 & 168217.100 & $9.690 \mathrm{E}-02$ & 4 & 6 & $1.161 \mathrm{E}-01$ & $1.437 \mathrm{E}+01$ & $5.835 \mathrm{E}+06$ \\
\hline & 157579.680 & 168397.890 & $9.860 \mathrm{E}-02$ & 4 & 4 & $3.499 \mathrm{E}-02$ & $4.258 \mathrm{E}+00$ & $2.732 E+06$ \\
\hline & 157579.680 & 168490.430 & $9.940 \mathrm{E}-02$ & 4 & 2 & $1.102 \mathrm{E}-01$ & $1.331 \mathrm{E}+01$ & $1.750 \mathrm{E}+07$ \\
\hline & 157677.320 & 168397.890 & $9.770 \mathrm{E}-02$ & 2 & 4 & $2.167 \mathrm{E}-01$ & $1.331 \mathrm{E}+01$ & $8.307 \mathrm{E}+06$ \\
\hline & 157677.320 & 168490.430 & $9.850 \mathrm{E}-02$ & 2 & 2 & $4.369 \mathrm{E}-02$ & $2.661 \mathrm{E}+00$ & $3.405 E+06$ \\
\hline
\end{tabular}


Table IV. Continued

\begin{tabular}{|c|c|c|c|c|c|c|c|c|}
\hline Transition & $\begin{array}{l}E_{i} \\
\mathrm{~cm}^{-1}\end{array}$ & $\begin{array}{l}E_{f} \\
\mathrm{~cm}^{-1}\end{array}$ & $\begin{array}{l}E_{f i} \\
\mathrm{Ry}\end{array}$ & $g_{i}$ & $g_{f}$ & $f_{i f}$ & $s$ & $\begin{array}{l}A_{f i} \\
\mathrm{~s}^{-1}\end{array}$ \\
\hline \multirow[t]{8}{*}{$\mathrm{y}^{4} \mathrm{P}^{\circ} \rightarrow \mathrm{h}^{4} \mathrm{P}^{\circ}$} & 0.2825 & 0.1369 & $1.456 \mathrm{E}-01$ & 12 & 12 & $3.767 \mathrm{E}-02$ & $9.317 \mathrm{E}+00$ & $6.413 E+06$ \\
\hline & 158038.600 & 174098.100 & $1.463 \mathrm{E}-01$ & 6 & 6 & $2.650 \mathrm{E}-02$ & $3.261 \mathrm{E}+00$ & $4.556 \mathrm{E}+06$ \\
\hline & 158038.600 & 173595.770 & $1.417 \mathrm{E}-01$ & 6 & 4 & $1.100 \mathrm{E}-02$ & $1.398 E+00$ & $2.661 \mathrm{E}+06$ \\
\hline & 157579.680 & 174098.100 & $1.505 \mathrm{E}-01$ & 4 & 6 & $1.753 \mathrm{E}-02$ & $1.398 \mathrm{E}+00$ & $2.126 \mathrm{E}+06$ \\
\hline & 157579.680 & 173595.770 & $1.459 \mathrm{E}-01$ & 4 & 4 & $5.035 \mathrm{E}-03$ & $4.141 \mathrm{E}-01$ & $8.608 \mathrm{E}+05$ \\
\hline & 157579.680 & 173316.830 & $1.434 \mathrm{E}-01$ & 4 & 2 & $1.546 \mathrm{E}-02$ & $1.294 \mathrm{E}+00$ & $5.108 \mathrm{E}+06$ \\
\hline & 157677.320 & 173595.770 & $1.450 \mathrm{E}-01$ & 2 & 4 & $3.127 \mathrm{E}-02$ & $1.294 \mathrm{E}+00$ & $2.641 \mathrm{E}+06$ \\
\hline & 157677.320 & 173316.830 & $1.425 \mathrm{E}-01$ & 2 & 2 & $6.146 \mathrm{E}-03$ & $2.588 \mathrm{E}-01$ & $1.003 \mathrm{E}+06$ \\
\hline \multirow[t]{8}{*}{$\mathrm{y}^{4} \mathrm{P}^{\mathrm{o}} \rightarrow \mathrm{i}^{4} \mathrm{P}^{\mathrm{e}}$} & 0.2825 & 0.1263 & $1.562 \mathrm{E}-01$ & 12 & 12 & $4.286 \mathrm{E}-02$ & $9.877 \mathrm{E}+00$ & $8.399 E+06$ \\
\hline & 158038.600 & 174892.750 & $1.535 \mathrm{E}-01$ & 6 & 6 & $2.948 \mathrm{E}-02$ & $3.457 \mathrm{E}+00$ & $5.579 \mathrm{E}+06$ \\
\hline & 158038.600 & 175019.780 & $1.547 \mathrm{E}-01$ & 6 & 4 & $1.273 \mathrm{E}-02$ & $1.482 \mathrm{E}+00$ & $3.672 \mathrm{E}+06$ \\
\hline & 157579.680 & 174892.750 & $1.577 \mathrm{E}-01$ & 4 & 6 & $1.947 \mathrm{E}-02$ & $1.482 \mathrm{E}+00$ & $2.593 E+06$ \\
\hline & 157579.680 & 175019.780 & $1.589 \mathrm{E}-01$ & 4 & 4 & $5.813 E-03$ & $4.390 \mathrm{E}-01$ & $1.179 \mathrm{E}+06$ \\
\hline & 157579.680 & 175083.510 & $1.595 \mathrm{E}-01$ & 4 & 2 & $1.823 \mathrm{E}-02$ & $1.372 E+00$ & $7.452 \mathrm{E}+06$ \\
\hline & 157677.320 & 175019.780 & $1.580 \mathrm{E}-01$ & 2 & 4 & $3.612 \mathrm{E}-02$ & $1.372 \mathrm{E}+00$ & $3.622 \mathrm{E}+06$ \\
\hline & 157677.320 & 175083.510 & $1.586 \mathrm{E}-01$ & 2 & 2 & $7.252 \mathrm{E}-03$ & $2.744 \mathrm{E}-01$ & $1.465 \mathrm{E}+06$ \\
\hline \multirow[t]{8}{*}{$y^{4} P^{o} \rightarrow j^{4} P^{e}$} & 0.2825 & 0.0905 & $1.920 \mathrm{E}-01$ & 12 & 12 & $1.486 \mathrm{E}-02$ & $2.786 \mathrm{E}+00$ & $4.398 \mathrm{E}+06$ \\
\hline & 158038.600 & 178848.960 & $1.896 \mathrm{E}-01$ & 6 & 6 & $1.027 \mathrm{E}-02$ & $9.752 \mathrm{E}-01$ & $2.966 \mathrm{E}+06$ \\
\hline & 158038.600 & 178915.200 & $1.902 \mathrm{E}-01$ & 6 & 4 & $4.416 \mathrm{E}-03$ & $4.180 \mathrm{E}-01$ & $1.925 \mathrm{E}+06$ \\
\hline & 157579.680 & 178848.960 & $1.938 \mathrm{E}-01$ & 4 & 6 & $6.750 \mathrm{E}-03$ & $4.180 \mathrm{E}-01$ & $1.358 \mathrm{E}+06$ \\
\hline & 157579.680 & 178915.200 & $1.944 \mathrm{E}-01$ & 4 & 4 & $2.006 \mathrm{E}-03$ & $1.238 \mathrm{E}-01$ & $6.090 \mathrm{E}+05$ \\
\hline & 157579.680 & 178969.320 & $1.949 \mathrm{E}-01$ & 4 & 2 & $6.285 \mathrm{E}-03$ & $3.870 \mathrm{E}-01$ & $3.836 \mathrm{E}+06$ \\
\hline & 157677.320 & 178915.200 & $1.935 \mathrm{E}-01$ & 2 & 4 & $1.248 \mathrm{E}-02$ & $3.870 \mathrm{E}-01$ & $1.877 \mathrm{E}+06$ \\
\hline & 157677.320 & 178969.320 & $1.940 \mathrm{E}-01$ & 2 & 2 & $2.503 \mathrm{E}-03$ & $7.740 \mathrm{E}-02$ & $7.565 \mathrm{E}+05$ \\
\hline \multirow[t]{9}{*}{$y^{4} P^{\circ} \rightarrow c^{4} D^{6}$} & 0.2825 & 0.1950 & $8.746 \mathrm{E}-02$ & 12 & 20 & $9.599 \mathrm{E}-01$ & $3.951 E+02$ & $3.539 \mathrm{E}+07$ \\
\hline & 158038.600 & 167575.230 & $8.690 \mathrm{E}-02$ & 6 & 8 & $7.630 \mathrm{E}-01$ & $1.580 \mathrm{E}+02$ & $3.471 \mathrm{E}+07$ \\
\hline & 158038.600 & 167364.980 & $8.490 \mathrm{E}-02$ & 6 & 6 & $1.677 \mathrm{E}-01$ & $3.556 \mathrm{E}+01$ & $9.711 \mathrm{E}+06$ \\
\hline & 158038.600 & 167291.840 & $8.430 \mathrm{E}-02$ & 6 & 4 & $1.850 \mathrm{E}-02$ & $3.951 \mathrm{E}+\infty 0$ & $1.584 \mathrm{E}+06$ \\
\hline & 157579.680 & 167364.980 & $8.910 \mathrm{E}-02$ & 4 & 6 & $6.161 \mathrm{E}-01$ & $8.297 \mathrm{E}+01$ & $2.619 E+07$ \\
\hline & 157579.680 & 167291.840 & $8.850 \mathrm{E}-02$ & 4 & 4 & $3.108 \mathrm{E}-01$ & $4.214 \mathrm{E}+01$ & $1.955 \mathrm{E}+07$ \\
\hline & 157579.680 & 167254.840 & $8.810 \mathrm{E}-02$ & 4 & 2 & $4.835 \mathrm{E}-02$ & $6.585 \mathrm{E}+00$ & $6.028 \mathrm{E}+06$ \\
\hline & 157677.320 & 167291.840 & $8.760 \mathrm{E}-02$ & 2 & 4 & $4.807 \mathrm{E}-01$ & $3.293 \mathrm{E}+01$ & $1.481 \mathrm{E}+07$ \\
\hline & 157677.320 & 167254.840 & $8.720 \mathrm{E}-02$ & 2 & 2 & $4.785 \mathrm{E}-01$ & $3.293 \mathrm{E}+01$ & $2.923 E+07$ \\
\hline \multirow{9}{*}{$\begin{array}{c}\mathrm{y}^{4} \mathrm{P}^{\circ} \rightarrow \mathrm{d}^{4} \mathrm{D}^{\mathrm{e}} \\
*\end{array}$} & 0.2825 & 0.1319 & $1.506 \mathrm{E}-01$ & 12 & 20 & $1.044 \mathrm{E}-01$ & $2.497 \mathrm{E}+01$ & $1.141 \mathrm{E}+07$ \\
\hline & & & & 6 & 8 & $8.354 \mathrm{E}-02$ & $9.987 \mathrm{E}+00$ & $1.141 \mathrm{E}+07$ \\
\hline & 158038.600 & 174360.920 & $1.487 \mathrm{E}-01$ & 6 & 6 & $1.856 \mathrm{E}-02$ & $2.247 \mathrm{E}+00$ & $3.297 \mathrm{E}+06$ \\
\hline & 158038.600 & 174329.310 & $1.484 \mathrm{E}-01$ & 6 & 4 & $2.058 \mathrm{E}-03$ & $2.497 \mathrm{E}-01$ & $5.462 \mathrm{E}+05$ \\
\hline & 157579.680 & 174360.920 & $1.529 \mathrm{E}-01$ & 4 & 6 & $6.681 \mathrm{E}-02$ & $5.243 E+00$ & $8.363 E+06$ \\
\hline & 157579.680 & 174329.310 & $1.526 \mathrm{E}-01$ & 4 & 4 & $3.387 \mathrm{E}-02$ & $2.663 \mathrm{E}+00$ & $6.335 \mathrm{E}+06$ \\
\hline & & & & 4 & 2 & $5.221 \mathrm{E}-03$ & $4.161 \mathrm{E}-01$ & $1.902 \mathrm{E}+06$ \\
\hline & 157677.320 & 174329.310 & $1.517 \mathrm{E}-01$ & 2 & 4 & $5.261 \mathrm{E}-02$ & $2.081 \mathrm{E}+00$ & $4.862 \mathrm{E}+06$ \\
\hline & & & & 2 & 2 & $5.221 \mathrm{E}-02$ & $2.081 \mathrm{E}+00$ & $9.508 \mathrm{E}+06$ \\
\hline \multirow{9}{*}{$\begin{array}{c}\mathrm{y}^{4} \mathrm{p}^{\circ} \rightarrow \mathrm{e}^{4} \mathrm{D}^{\mathrm{e}} \\
*\end{array}$} & 0.2825 & 0.0955 & $1.870 \mathrm{E}-01$ & 12 & 20 & $3.273 \mathrm{E}-02$ & $6.301 \mathrm{E}+00$ & $5.515 \mathrm{E}+06$ \\
\hline & & & & 6 & 8 & $2.618 \mathrm{E}-02$ & $2.521 \mathrm{E}+00$ & $5.515 \mathrm{E}+06$ \\
\hline & 158038.600 & 178344.360 & $1.850 \mathrm{E}-01$ & 6 & 6 & $5.829 \mathrm{E}-03$ & $5.671 \mathrm{E}-01$ & $1.602 E+06$ \\
\hline & & & & 6 & 4 & $6.546 \mathrm{E}-04$ & $6.301 \mathrm{E}-02$ & $2.757 \mathrm{E}+05$ \\
\hline & 157579.680 & 178344.360 & $1.892 \mathrm{E}-01$ & 4 & 6 & $2.086 \mathrm{E}-02$ & $1.323 E+00$ & $3.999 \mathrm{E}+06$ \\
\hline & & & & 4 & 4 & $1.047 \mathrm{E}-02$ & $6.722 \mathrm{E}-01$ & $2.941 \mathrm{E}+06$ \\
\hline & & & & 4 & 2 & $1.636 \mathrm{E}-03$ & $1.050 \mathrm{E}-01$ & $9.191 \mathrm{E}+05$ \\
\hline & & & & 2 & 4 & $1.636 \mathrm{E}-02$ & $5.251 \mathrm{E}-01$ & $2.298 \mathrm{E}+06$ \\
\hline & & & & 2 & 2 & $1.636 \mathrm{E}-02$ & $5.251 \mathrm{E}-01$ & $4.596 \mathrm{E}+06$ \\
\hline \multirow[t]{9}{*}{$z^{4} D^{0} \rightarrow c^{4} P^{0}$} & 0.5517 & 0.5294 & $2.228 \mathrm{E}-02$ & 20 & 12 & $2.144 \mathrm{E}-04$ & $5.773 \mathrm{E}-01$ & $1.424 \mathrm{E}+03$ \\
\hline & 128599.160 & 130602.210 & $1.820 \mathrm{E}-02$ & 8 & 6 & $1.751 \mathrm{E}-04$ & $2.309 \mathrm{E}-01$ & $6.212 \mathrm{E}+02$ \\
\hline & 128233.200 & 130602.210 & $2.160 \mathrm{E}-02$ & 6 & 6 & $6.235 \mathrm{E}-05$ & $5.196 \mathrm{E}-02$ & $2.337 \mathrm{E}+02$ \\
\hline & 127976.340 & 130602.210 & $2.390 \mathrm{E}-02$ & 4 & 6 & $1.150 \mathrm{E}-05$ & $5.773 \mathrm{E}-03$ & $3.517 \mathrm{E}+01$ \\
\hline & 128233.200 & 130818.850 & $2.360 \mathrm{E}-02$ & 6 & 4 & $1.590 \mathrm{E}-04$ & $1.212 \mathrm{E}-01$ & $1.067 \mathrm{E}+03$ \\
\hline & 127976.340 & 130818.850 & $2.590 \mathrm{E}-02$ & 4 & 4 & $1.329 \mathrm{E}-04$ & $6.158 \mathrm{E}-02$ & $7.161 \mathrm{E}+02$ \\
\hline & 127825.080 & 130818.850 & $2.730 \mathrm{E}-02$ & 2 & 4 & $4.378 \mathrm{E}-05$ & $9.622 \mathrm{E}-03$ & $1.310 \mathrm{E}+02$ \\
\hline & 127976.340 & 130948.940 & $2.710 \mathrm{E}-02$ & 4 & 2 & $1.086 \mathrm{E}-04$ & $4.811 \mathrm{E}-02$ & $1.282 \mathrm{E}+03$ \\
\hline & 127825.080 & 130948.940 & $2.850 \mathrm{E}-02$ & 2 & 2 & $2.285 \mathrm{E}-04$ & $4.811 \mathrm{E}-02$ & $1.491 \mathrm{E}+03$ \\
\hline
\end{tabular}


Table IV. Continued

\begin{tabular}{|c|c|c|c|c|c|c|c|c|}
\hline Transition & $\begin{array}{l}E_{i} \\
\mathrm{~cm}^{-1}\end{array}$ & $\begin{array}{l}E_{f} \\
\mathrm{~cm}^{-1}\end{array}$ & $\begin{array}{l}E_{f i} \\
\mathrm{Ry}\end{array}$ & $g_{i}$ & $g_{f}$ & $f_{i f}$ & $S$ & $\begin{array}{l}A_{f i} \\
\mathrm{~s}^{-1}\end{array}$ \\
\hline \multirow[t]{9}{*}{$z^{4} D^{\circ} \rightarrow d^{4} P^{e}$} & 0.5517 & 0.3473 & $2.044 \mathrm{E}-01$ & 20 & 12 & $2.188 \mathrm{E}-01$ & $6.421 \mathrm{E}+01$ & $1.224 \mathrm{E}+08$ \\
\hline & 128599.160 & 150996.410 & $2.041 \mathrm{E}-01$ & 8 & 6 & $2.184 \mathrm{E}-01$ & $2.568 \mathrm{E}+01$ & $9.745 \mathrm{E}+07$ \\
\hline & 128233.200 & 150996.410 & $2.075 \mathrm{E}-01$ & 6 & 6 & $6.662 \mathrm{E}-02$ & $5.779 \mathrm{E}+00$ & $2.304 \mathrm{E}+07$ \\
\hline & 127976.340 & 150996.410 & $2.098 E-01$ & 4 & 6 & $1.123 \mathrm{E}-02$ & $6.421 \mathrm{E}-01$ & $2.646 \mathrm{E}+06$ \\
\hline & 128233.200 & 150531.310 & $2.032 E-01$ & 6 & 4 & $1.522 \mathrm{E}-01$ & $1.348 \mathrm{E}+01$ & $7.573 \mathrm{E}+07$ \\
\hline & 127976.340 & 150531.310 & $2.055 \mathrm{E}-01$ & 4 & 4 & $1.173 \mathrm{E}-01$ & $6.849 \mathrm{E}+00$ & $3.979 E+07$ \\
\hline & 127825.080 & 150531.310 & $2.069 \mathrm{E}-01$ & 2 & 4 & $3.690 \mathrm{E}-02$ & $1.070 \mathrm{E}+00$ & $6.344 \mathrm{E}+06$ \\
\hline & 127976.340 & 150258.510 & $2.031 E-01$ & 4 & 2 & $9.056 \mathrm{E}-02$ & $5.351 \mathrm{E}+00$ & $6.001 E+07$ \\
\hline & 127825.080 & 150258.510 & $2.045 \mathrm{E}-01$ & 2 & 2 & $1.824 \mathrm{E}-01$ & $5.351 \mathrm{E}+00$ & $6.126 \mathrm{E}+07$ \\
\hline \multirow[t]{9}{*}{$\mathrm{z}^{4} \mathrm{D}^{\circ} \rightarrow \mathrm{e}^{4} \mathrm{P}^{\mathrm{e}}$} & 0.5517 & 0.2996 & $2.520 \mathrm{E}-01$ & 20 & 12 & $6.271 \mathrm{E}-03$ & $1.493 \mathrm{E}+00$ & $5.332 \mathrm{E}+06$ \\
\hline & 128599.160 & 155818.710 & $2.480 \mathrm{E}-01$ & 8 & 6 & $6.170 E-03$ & $5.971 \mathrm{E}-01$ & $4.064 \mathrm{E}+06$ \\
\hline & 128233.200 & 155818.710 & $2.514 \mathrm{E}-01$ & 6 & 6 & $1.876 \mathrm{E}-03$ & $1.344 \mathrm{E}-01$ & $9.526 \mathrm{E}+05$ \\
\hline & 127976.340 & 155818.710 & $2.537 \mathrm{E}-01$ & 4 & 6 & $3.156 \mathrm{E}-04$ & $1.493 \mathrm{E}-02$ & $1.088 \mathrm{E}+05$ \\
\hline & 128233.200 & 156029.540 & $2.533 \mathrm{E}-01$ & 6 & 4 & $4.412 \mathrm{E}-03$ & $3.135 \mathrm{E}-01$ & $3.410 \mathrm{E}+06$ \\
\hline & 127976.340 & 156029.540 & $2.556 \mathrm{E}-01$ & 4 & 4 & $3.392 \mathrm{E}-03$ & $1.592 \mathrm{E}-01$ & $1.780 E+06$ \\
\hline & 127825.080 & 156029.540 & $2.570 \mathrm{E}-01$ & 2 & 4 & $1.066 \mathrm{E}-03$ & $2.488 \mathrm{E}-02$ & $2.827 \mathrm{E}+05$ \\
\hline & 127976.340 & 156148.480 & $2.567 \mathrm{E}-01$ & 4 & 2 & $2.661 \mathrm{E}-03$ & $1.244 \mathrm{E}-01$ & $2.817 \mathrm{E}+06$ \\
\hline & 127825.080 & 156148.480 & $2.581 \mathrm{E}-01$ & 2 & 2 & $5.351 \mathrm{E}-03$ & $1.244 \mathrm{E}-01$ & $2.863 \mathrm{E}+06$ \\
\hline \multirow[t]{9}{*}{$z^{4} D^{\circ} \rightarrow f^{4} P^{e}$} & 0.5517 & 0.2063 & $3.454 \mathrm{E}-01$ & 20 & 12 & $2.721 \mathrm{E}-02$ & $4.726 \mathrm{E}+00$ & $4.345 \mathrm{E}+07$ \\
\hline & 128599.160 & 166479.820 & $3.452 \mathrm{E}-01$ & 8 & 6 & $2.719 \mathrm{E}-02$ & $1.891 E+00$ & $3.470 \mathrm{E}+07$ \\
\hline & 128233.200 & 166479.820 & $3.486 \mathrm{E}-01$ & 6 & 6 & $8.238 \mathrm{E}-03$ & $4.254 \mathrm{E}-01$ & $8.041 \mathrm{E}+06$ \\
\hline & 127976.340 & 166479.820 & $3.509 \mathrm{E}-01$ & 4 & 6 & $1.382 \mathrm{E}-03$ & $4.726 \mathrm{E}-02$ & $9.112 \mathrm{E}+05$ \\
\hline & 128233.200 & 165991.350 & $3.441 \mathrm{E}-01$ & 6 & 4 & $1.897 \mathrm{E}-02$ & $9.925 \mathrm{E}-01$ & $2.707 \mathrm{E}+07$ \\
\hline & 127976.340 & 165991.350 & $3.464 \mathrm{E}-01$ & 4 & 4 & $1.455 \mathrm{E}-02$ & $5.041 \mathrm{E}-01$ & $1.403 E+07$ \\
\hline & 127825.080 & 165991.350 & $3.478 \mathrm{E}-01$ & 2 & 4 & $4.566 \mathrm{E}-03$ & $7.877 \mathrm{E}-02$ & $2.218 \mathrm{E}+06$ \\
\hline & 127976.340 & 165719.230 & $3.439 \mathrm{E}-01$ & 4 & 2 & $1.129 \mathrm{E}-02$ & $3.939 E-01$ & $2.144 \mathrm{E}+07$ \\
\hline & 127825.080 & 165719.230 & $3.453 \mathrm{E}-01$ & 2 & 2 & $2.267 \mathrm{E}-02$ & $3.939 \mathrm{E}-01$ & $2.171 \mathrm{E}+07$ \\
\hline \multirow[t]{9}{*}{$z^{4} D^{\circ} \rightarrow g^{4} P^{e}$} & 0.5517 & 0.1868 & $3.648 \mathrm{E}--01$ & 20 & 12 & $1.241 \mathrm{E}-03$ & $2.041 \mathrm{E}-01$ & $2.211 \mathrm{E}+06$ \\
\hline & 128599.160 & 168217.100 & $3.610 \mathrm{E}-01$ & 8 & 6 & $1.228 \mathrm{E}-03$ & $8.163 E-02$ & $1.714 \mathrm{E}+06$ \\
\hline & 128233.200 & 168217.100 & $3.644 E-01$ & 6 & 6 & $3.718 \mathrm{E}-04$ & $1.837 \mathrm{E}-02$ & $3.966 \mathrm{E}+05$ \\
\hline & 127976.340 & 168217.100 & $3.667 \mathrm{E}-01$ & 4 & 6 & $6.236 \mathrm{E}-05$ & $2.041 \mathrm{E}-03$ & $4.490 \mathrm{E}+04$ \\
\hline & 128233.200 & 168397.890 & $3.661 \mathrm{E}-01$ & 6 & 4 & $8.716 \mathrm{E}-04$ & $4.286 \mathrm{E}-02$ & $1.408 \mathrm{E}+06$ \\
\hline & 127976.340 & 168397.890 & $3.684 \mathrm{E}-01$ & 4 & 4 & $6.683 \mathrm{E}-04$ & $2.177 \mathrm{E}-02$ & $7.285 E+05$ \\
\hline & 127825.080 & 168397.890 & $3.698 \mathrm{E}-01$ & 2 & 4 & $2.096 \mathrm{E}-04$ & $3.401 E-03$ & $1.151 \mathrm{E}+05$ \\
\hline & 127976.340 & 168490.430 & $3.692 \mathrm{E}-01$ & 4 & 2 & $5.232 \mathrm{E}-04$ & $1.701 \mathrm{E}-02$ & $1.146 \mathrm{E}+06$ \\
\hline & 127825.080 & 168490.430 & $3.706 \mathrm{E}-01$ & 2 & 2 & $1.050 \mathrm{E}-03$ & $1.701 \mathrm{E}-02$ & $1.159 \mathrm{E}+06$ \\
\hline \multirow[t]{9}{*}{$z^{4} D^{\circ} \rightarrow b^{4} P^{e}$} & 0.5517 & 0.1369 & $4.147 \mathrm{E}-01$ & 20 & 12 & $9.652 \mathrm{E}-03$ & $1.396 \mathrm{E}+\infty$ & $2.223 \mathrm{E}+07$ \\
\hline & 128599.160 & 174098.100 & $4.146 \mathrm{E}-01$ & 8 & 6 & $9.649 \mathrm{E}-03$ & $5.585 \mathrm{E}-01$ & $1.776 \mathrm{E}+07$ \\
\hline & 128233.200 & 174098.100 & $4.180 \mathrm{E}-01$ & 6 & 6 & $2.918 \mathrm{E}-03$ & $1.257 \mathrm{E}-01$ & $4.096 \mathrm{E}+06$ \\
\hline & 127976.340 & 174098.100 & $4.203 \mathrm{E}-01$ & 4 & 6 & $4.891 \mathrm{E}-04$ & $1.396 \mathrm{E}-02$ & $4.626 \mathrm{E}+05$ \\
\hline & 128233.200 & 173595.770 & $4.134 \mathrm{E}-01$ & 6 & 4 & $6.735 \mathrm{E}-03$ & $2.932 \mathrm{E}-01$ & $1.387 \mathrm{E}+07$ \\
\hline & 127976.340 & 173595.770 & $4.157 \mathrm{E}-01$ & 4 & 4 & $5.160 \mathrm{E}-03$ & $1.489 \mathrm{E}-01$ & $7.162 E+06$ \\
\hline & 127825.080 & 173595.770 & $4.171 \mathrm{E}-01$ & 2 & 4 & $1.618 \mathrm{E}-03$ & $2.327 \mathrm{E}-02$ & $1.130 \mathrm{E}+06$ \\
\hline & 127976.340 & 173316.830 & $4.132 \mathrm{E}-01$ & 4 & 2 & $4.007 \mathrm{E}-03$ & $1.164 \mathrm{E}-01$ & $1.099 \mathrm{E}+07$ \\
\hline & 127825.080 & 173316.830 & $4.146 \mathrm{E}-01$ & 2 & 2 & $8.041 \mathrm{E}-03$ & $1.164 \mathrm{E}-01$ & $1.110 \mathrm{E}+07$ \\
\hline \multirow[t]{9}{*}{$z^{4} D^{\circ} \rightarrow i^{4} P^{e}$} & 0.5517 & 0.1263 & $4.254 \mathrm{E}-01$ & 20 & 12 & $4.625 \mathrm{E}-04$ & $6.523 \mathrm{E}-02$ & $1.120 \mathrm{E}+06$ \\
\hline & 128599.160 & 174892.750 & $4.218 \mathrm{E}-01$ & 8 & 6 & $4.586 \mathrm{E}-04$ & $2.609 \mathrm{E}-02$ & $8.738 \mathrm{E}+05$ \\
\hline & 128233.200 & 174892.750 & $4.252 \mathrm{E}-01$ & 6 & 6 & $1.387 \mathrm{E}-04$ & $5.871 \mathrm{E}-03$ & $2.014 \mathrm{E}+05$ \\
\hline & 127976.340 & 174892.750 & $4.275 \mathrm{E}-01$ & 4 & 6 & $2.324 \mathrm{E}-05$ & $6.523 \mathrm{E}-04$ & $2.274 \mathrm{E}+04$ \\
\hline & 128233.200 & 175019.780 & $4.264 \mathrm{E}-01$ & 6 & 4 & $3.245 \mathrm{E}-04$ & $1.370 \mathrm{E}-02$ & $7.108 \mathrm{E}+05$ \\
\hline & 127976.340 & 175019.780 & $4.287 \mathrm{E}-01$ & 4 & 4 & $2.486 \mathrm{E}-04$ & $6.958 \mathrm{E}-03$ & $3.669 E+05$ \\
\hline & 127825.080 & 175019.780 & $4.301 \mathrm{E}-01$ & 2 & 4 & $7.793 E-05$ & $1.087 \mathrm{E}-03$ & $5.790 \mathrm{E}+04$ \\
\hline & 127976.340 & 175083.510 & $4.293 E-01$ & 4 & 2 & $1.945 \mathrm{E}-04$ & $5.436 \mathrm{E}-03$ & $5.757 \mathrm{E}+05$ \\
\hline & 127825.080 & 175083.510 & $4.307 \mathrm{E}-01$ & 2 & 2 & $3.902 \mathrm{E}-04$ & $5.436 \mathrm{E}-03$ & $5.814 \mathrm{E}+05$ \\
\hline
\end{tabular}


Table IV. Continued

\begin{tabular}{|c|c|c|c|c|c|c|c|c|}
\hline Transition & $\begin{array}{l}E_{i} \\
\mathrm{~cm}^{-1}\end{array}$ & $\begin{array}{l}E_{f} \\
\mathrm{~cm}^{-1}\end{array}$ & $\begin{array}{l}E_{f i} \\
\mathrm{Ry}\end{array}$ & $g_{i}$ & $g_{f}$ & $f_{i f}$ & $S$ & $\begin{array}{l}A_{f i} \\
\mathrm{~s}^{-i} \\
\end{array}$ \\
\hline \multirow[t]{9}{*}{$z^{4} D^{0} \rightarrow j^{4} P^{e}$} & 0.5517 & 0.0905 & $4.611 \mathrm{E}-01$ & 20 & 12 & $2.250 \mathrm{E}-04$ & $2.927 \mathrm{E}-02$ & $6.404 \mathrm{E}+05$ \\
\hline & 128599.160 & 178848.960 & $4.579 \mathrm{E}-01$ & 8 & 6 & $2.234 \mathrm{E}-04$ & $1.171 \mathrm{E}-02$ & $5.016 \mathrm{E}+05$ \\
\hline & 128233.200 & 178848.960 & $4.613 \mathrm{E}-01$ & 6 & 6 & $6.751 \mathrm{E}-05$ & $2.634 \mathrm{E}-03$ & $1.154 \mathrm{E}+05$ \\
\hline & 127976.340 & 178848.960 & $4.636 \mathrm{E}-01$ & 4 & 6 & $1.131 \mathrm{E}-05$ & $2.927 \mathrm{E}-04$ & $1.301 \mathrm{E}+04$ \\
\hline & 128233.200 & 178915.200 & $4.619 \mathrm{E}-01$ & 6 & 4 & $1.577 \mathrm{E}-04$ & $6.147 \mathrm{E}-03$ & $4.055 \mathrm{E}+05$ \\
\hline & 127976.340 & 178915.200 & $4.642 \mathrm{E}-01$ & 4 & 4 & $1.208 \mathrm{E}-04$ & $3.122 \mathrm{E}-03$ & $2.090 \mathrm{E}+05$ \\
\hline & 127825.080 & 178915.200 & $4.656 \mathrm{E}-01$ & 2 & 4 & $3.786 \mathrm{E}-05$ & $4.879 \mathrm{E}-04$ & $3.296 \mathrm{E}+04$ \\
\hline & 127976.340 & 178969.320 & $4.647 \mathrm{E}-01$ & 4 & 2 & $9.446 \mathrm{E}-05$ & $2.439 \mathrm{E}-03$ & $3.277 \mathrm{E}+05$ \\
\hline & 127825.080 & 178969.320 & $4.661 \mathrm{E}-01$ & 2 & 2 & $1.895 \mathrm{E}-04$ & $2.439 \mathrm{E}-03$ & $3.307 \mathrm{E}+05$ \\
\hline \multirow[t]{11}{*}{$z^{4} D^{\circ} \rightarrow b^{4} D^{e}$} & 0.5517 & 0.3237 & $2.280 \mathrm{E}-01$ & 20 & 20 & $1.478 \mathrm{E}-01$ & $3.890 \mathrm{E}+01$ & $6.172 \mathrm{E}+07$ \\
\hline & 128599.160 & 153413.740 & $2.261 \mathrm{E}-01$ & 8 & 8 & $1.257 \mathrm{E}-01$ & $1.334 \mathrm{E}+01$ & $5.162 \mathrm{E}+07$ \\
\hline & 128599.160 & 153283.070 & $2.249 \mathrm{E}-01$ & 8 & 6 & $2.078 \mathrm{E}-02$ & $2.218 \mathrm{E}+00$ & $1.126 \mathrm{E}+07$ \\
\hline & 128233.200 & 153413.740 & $2.295 \mathrm{E}-01$ & 6 & 8 & $2.827 \mathrm{E}-02$ & $2.218 \mathrm{E}+\infty 0$ & $8.971 \mathrm{E}+06$ \\
\hline & 128233.200 & 153283.070 & $2.283 \mathrm{E}-01$ & 6 & 6 & $8.487 \mathrm{E}-02$ & $6.691 \mathrm{E}+\infty 0$ & $3.553 \mathrm{E}+07$ \\
\hline & 128233.200 & 153201.950 & $2.276 \mathrm{E}-01$ & 6 & 4 & $3.443 \mathrm{E}-02$ & $2.723 \mathrm{E}+\infty 0$ & $2.149 \mathrm{E}+07$ \\
\hline & 127976.340 & 153283.070 & $2.306 \mathrm{E}-01$ & 4 & 6 & $5.233 \mathrm{E}-02$ & $2.723 \mathrm{E}+00$ & $1.490 \mathrm{E}+07$ \\
\hline & 127976.340 & 153201.950 & $2.299 \mathrm{E}-01$ & 4 & 4 & $5.963 \mathrm{E}-02$ & $3.112 \mathrm{E}+\infty 0$ & $2.531 \mathrm{E}+07$ \\
\hline & 127976.340 & 153153.900 & $2.294 \mathrm{E}-01$ & 4 & 2 & $3.719 \mathrm{E}-02$ & $1.945 \mathrm{E}+00$ & $3.144 \mathrm{E}+07$ \\
\hline & 127825.080 & 153201.950 & $2.313 \mathrm{E}-01$ & 2 & 4 & $7.499 \mathrm{E}-02$ & $1.945 \mathrm{E}+00$ & $1.611 \mathrm{E}+07$ \\
\hline & 127825.080 & 153153.900 & $2.308 \mathrm{E}-01$ & 2 & 2 & $7.482 \mathrm{E}-02$ & $1.945 \mathrm{E}+00$ & $3.202 \mathrm{E}+07$ \\
\hline \multirow[t]{11}{*}{$z^{4} D^{\circ} \rightarrow c^{4} D^{e}$} & 0.5517 & 0.1950 & $3.566 \mathrm{E}-01$ & 20 & 20 & $1.483 \mathrm{E}-02$ & $2.496 \mathrm{E}+00$ & $1.515 \mathrm{E}+07$ \\
\hline & 128599.160 & 167575.230 & $3.552 \mathrm{E}-01$ & 8 & 8 & $1.267 \mathrm{E}-02$ & $8.560 \mathrm{E}-01$ & $1.284 \mathrm{E}+07$ \\
\hline & 128599.160 & 167364.980 & $3.532 \mathrm{E}-01$ & 8 & 6 & $2.094 \mathrm{E}-03$ & $1.423 \mathrm{E}-01$ & $2.797 \mathrm{E}+06$ \\
\hline & 128233.200 & 167575.230 & $3.586 \mathrm{E}-01$ & 6 & 8 & $2.834 \mathrm{E}-03$ & $1.423 \mathrm{E}-01$ & $2.195 \mathrm{E}+06$ \\
\hline & 128233.200 & 167364.980 & $3.566 \mathrm{E}-01$ & 6 & 6 & $8.504 E-03$ & $4.293 \mathrm{E}-01$ & $8.686 \mathrm{E}+06$ \\
\hline & 128233.200 & 167291.840 & $3.560 \mathrm{E}-01$ & 6 & 4 & $3.455 \mathrm{E}-03$ & $1.747 \mathrm{E}-01$ & $5.276 \mathrm{E}+06$ \\
\hline & 127976.340 & 167364.980 & $3.589 \mathrm{E}-01$ & 4 & 6 & $5.225 \mathrm{E}-03$ & $1.747 \mathrm{E}-01$ & $3.604 \mathrm{E}+06$ \\
\hline & 127976.340 & 167291.840 & $3.583 \mathrm{E}-01$ & 4 & 4 & $5.962 \mathrm{E}-03$ & $1.997 \mathrm{E}-01$ & $6.147 \mathrm{E}+06$ \\
\hline & 127976.340 & 167254.840 & $3.579 \mathrm{E}-01$ & 4 & 2 & $3.722 \mathrm{E}-03$ & $1.248 \mathrm{E}-01$ & $7.658 \mathrm{E}+06$ \\
\hline & 127825.080 & 167291.840 & $3.597 \mathrm{E}-01$ & 2 & 4 & $7.481 \mathrm{E}-03$ & $1.248 \mathrm{E}-01$ & $3.887 E+06$ \\
\hline & 127825.080 & 167254.840 & $3.593 \mathrm{E}-01$ & 2 & 2 & $7.473 \mathrm{E}-03$ & $1.248 \mathrm{E}-01$ & $7.749 \mathrm{E}+06$ \\
\hline \multirow{11}{*}{$\begin{array}{c}\mathrm{z}^{4} \mathrm{D}^{\circ} \rightarrow \mathrm{d}^{4} \mathrm{D}^{\mathrm{e}} \\
*\end{array}$} & 0.5517 & 0.1319 & $4.197 \mathrm{E}-01$ & 20 & 20 & $4.330 \mathrm{E}-03$ & $6.190 \mathrm{E}-01$ & $6.127 \mathrm{E}+06$ \\
\hline & & & & 8 & 8 & $3.711 \mathrm{E}-03$ & $2.123 \mathrm{E}-01$ & $5.248 \mathrm{E}+06$ \\
\hline & 128599.160 & 174360.920 & $4.170 \mathrm{E}-01$ & 8 & 6 & $6.130 \mathrm{E}-04$ & $3.528 \mathrm{E}-02$ & $1.142 \mathrm{E}+06$ \\
\hline & & & & 6 & 8 & $8.248 \mathrm{E}-04$ & $3.528 \mathrm{E}-02$ & $8.797 \mathrm{E}+05$ \\
\hline & 128233.200 & 174360.920 & $4.204 \mathrm{E}-01$ & 6 & 6 & $2.486 \mathrm{E}-03$ & $1.065 \mathrm{E}-01$ & $3.530 \mathrm{E}+06$ \\
\hline & 128233.200 & 174329.310 & $4.201 \mathrm{E}-01$ & 6 & 4 & $1.011 \mathrm{E}-03$ & $4.333 \mathrm{E}-02$ & $2.150 \mathrm{E}+06$ \\
\hline & 127976.340 & 174360.920 & $4.227 \mathrm{E}-01$ & 4 & 6 & $1.526 \mathrm{E}-03$ & $4.333 \mathrm{E}-02$ & $1.460 \mathrm{E}+06$ \\
\hline & 127976.340 & 174329.310 & $4.224 \mathrm{E}-01$ & 4 & 4 & $1.743 \mathrm{E}-03$ & $4.952 \mathrm{E}-02$ & $2.498 \mathrm{E}+06$ \\
\hline & & & & 4 & 2 & $1.082 E-03$ & $3.095 \mathrm{E}-02$ & $3.064 \mathrm{E}+06$ \\
\hline & 127825.080 & 174329.310 & $4.238 \mathrm{E}-01$ & 2 & 4 & $2.186 \mathrm{E}-03$ & $3.095 \mathrm{E}-02$ & $1.577 \mathrm{E}+06$ \\
\hline & & & & 2 & 2 & $2.165 \mathrm{E}-03$ & $3.095 \mathrm{E}-02$ & $3.064 \mathrm{E}+06$ \\
\hline \multirow{11}{*}{$\begin{array}{c}z^{4} D^{\alpha \rightarrow} e^{4} D^{e} \\
*\end{array}$} & 0.5517 & 0.0955 & $4.562 \mathrm{E}-01$ & 20 & 20 & $1.876 \mathrm{E}-03$ & $2.468 \mathrm{E}-01$ & $3.135 \mathrm{E}+06$ \\
\hline & & & & 8 & 8 & $1.608 E-03$ & $8.464 \mathrm{E}-02$ & $2.685 E+06$ \\
\hline & 128599.160 & 178344.360 & $4.533 \mathrm{E}-01$ & 8 & 6 & $2.657 \mathrm{E}-04$ & $1.407 \mathrm{E}-02$ & $5.846 \mathrm{E}+05$ \\
\hline & & & & 6 & 8 & $3.573 \mathrm{E}-0.4$ & $1.407 \mathrm{E}-02$ & $4.502 \mathrm{E}+05$ \\
\hline & 128233.200 & 178344.360 & $4.567 \mathrm{E}-01$ & 6 & 6 & $1.077 \mathrm{E}-03$ & $4.244 \mathrm{E}-02$ & $1.804 \mathrm{E}+06$ \\
\hline & & & & 6 & 4 & $4.377 \mathrm{E}-04$ & $1.727 \mathrm{E}-02$ & $1.097 \mathrm{E}+06$ \\
\hline & 127976.340 & 178344.360 & $4.590 \mathrm{E}-01$ & 4 & 6 & $6.607 \mathrm{E}-04$ & $1.727 \mathrm{E}-02$ & $7.454 \mathrm{E}+05$ \\
\hline & & & & 4 & 4 & $7.504 \mathrm{E}-04$ & $1.974 \mathrm{E}-02$ & $1.254 \mathrm{E}+06$ \\
\hline & & & & 4 & 2 & $4.690 \mathrm{E}-04$ & $1.234 \mathrm{E}-02$ & $1.568 \mathrm{E}+06$ \\
\hline & & & & 2 & 4 & $9.380 \mathrm{E}-04$ & $1.234 \mathrm{E}-02$ & $7.838 \mathrm{E}+05$ \\
\hline & & & & 2 & 2 & $9.380 \mathrm{E}-04$ & $1.234 \mathrm{E}-02$ & $1.568 \mathrm{E}+06$ \\
\hline \multirow[t]{10}{*}{$z^{4} D^{\circ} \rightarrow b^{4} F^{*}$} & 0.5517 & 0.3326 & $2.190 \mathrm{E}-01$ & 20 & 28 & $8.821 \mathrm{E}-01$ & $2.417 \mathrm{E}+02$ & $2.428 \mathrm{E}+08$ \\
\hline & 128599.160 & 152615.460 & $2.188 \mathrm{E}-01$ & 8 & 10 & $7.869 \mathrm{E}-01$ & $8.631 \mathrm{E}+01$ & $2.421 \mathrm{E}+08$ \\
\hline & 128599.160 & 152305.000 & $2.160 \mathrm{E}-01$ & 8 & 8 & $8.855 \mathrm{E}-02$ & $9.839 \mathrm{E}+00$ & $3.319 E+07$ \\
\hline & 128599.160 & 152094.640 & $2.141 \mathrm{E}-01$ & 8 & 6 & $4.466 \mathrm{E}-03$ & $5.006 \mathrm{E}-01$ & $2.192 \mathrm{E}+06$ \\
\hline & 128233.200 & 152305.000 & $2.194 \mathrm{E}-01$ & 6 & 8 & $7.217 \mathrm{E}-01$ & $5.921 \mathrm{E}+01$ & $2.093 \mathrm{E}+08$ \\
\hline & 128233.200 & 152094.640 & $2.175 \mathrm{E}-01$ & 6 & 6 & $1.523 \mathrm{E}-01$ & $1.260 \mathrm{E}+01$ & $5.786 \mathrm{E}+07$ \\
\hline & 128233.200 & 151959.690 & $2.163 E-01$ & 6 & 4 & $8.297 \mathrm{E}-03$ & $6.905 E-01$ & $4.677 \mathrm{E}+06$ \\
\hline & 127976.340 & 152094.640 & $2.198 \mathrm{E}-01$ & 4 & 6 & $7.114 \mathrm{E}-01$ & $3.884 \mathrm{E}+01$ & $1.840 \mathrm{E}+08$ \\
\hline & 127976.340 & 151959.690 & $2.186 \mathrm{E}-01$ & 4 & 4 & $1.761 \mathrm{E}-01$ & $9.667 \mathrm{E}+00$ & $6.759 \mathrm{E}+07$ \\
\hline & 127825.080 & 151959.690 & $2.200 \mathrm{E}-01$ & 2 & 4 & $8.861 \mathrm{E}-01$ & $2.417 \mathrm{E}+01$ & $1.722 \mathrm{E}+08$ \\
\hline
\end{tabular}


Table IV. Continued

\begin{tabular}{|c|c|c|c|c|c|c|c|c|}
\hline Transition & $\begin{array}{l}E_{i} \\
\mathrm{~cm}^{-1}\end{array}$ & $\begin{array}{l}E_{f} \\
\mathrm{~cm}^{-1} \\
\end{array}$ & $\begin{array}{l}E_{f i} \\
\text { Ry }\end{array}$ & $g_{i}$ & $g_{f}$ & $f_{i f}$ & $S$ & $\begin{array}{l}A_{f i} \\
\mathrm{~s}^{-1}\end{array}$ \\
\hline \multirow[t]{10}{*}{$\mathrm{z}^{4} \mathrm{D}^{\circ} \rightarrow \mathrm{c}^{4} \mathrm{~F}^{\mathrm{e}}$} & 0.5517 & 0.1993 & $3.523 \mathrm{E}-01$ & 20 & 28 & $6.994 \mathrm{E}-02$ & $1.191 \mathrm{E}+01$ & $4.982 \mathrm{E}+07$ \\
\hline & 128599.160 & 167269.150 & $3.524 \mathrm{E}-01$ & 8 & 10 & $6.246 \mathrm{E}-02$ & $4.254 \mathrm{E}+00$ & $4.984 \mathrm{E}+07$ \\
\hline & 128599.160 & 166918.270 & $3.492 \mathrm{E}-01$ & 8 & 8 & $7.056 \mathrm{E}-03$ & $4.849 \mathrm{E}-01$ & $6.911 \mathrm{E}+06$ \\
\hline & 128599.160 & 166709.270 & $3.473 \mathrm{E}-01$ & 8 & 6 & $3.570 \mathrm{E}-04$ & $2.467 \mathrm{E}-02$ & $4.612 \mathrm{E}+05$ \\
\hline & 128233.200 & 166918.270 & $3.526 \mathrm{E}-01$ & 6 & 8 & $5.716 \mathrm{E}-02$ & $2.918 \mathrm{E}+00$ & $4.281 \mathrm{E}+07$ \\
\hline & 128233.200 & 166709.270 & $3.507 \mathrm{E}-01$ & 6 & 6 & $1.210 \mathrm{E}-02$ & $6.211 \mathrm{E}-01$ & $1.195 \mathrm{E}+07$ \\
\hline & 128233.200 & 166591.080 & $3.496 \mathrm{E}-01$ & 6 & 4 & $6.610 \mathrm{E}-04$ & $3.403 \mathrm{E}-02$ & $9.733 E+05$ \\
\hline & 127976.340 & 166709.270 & $3.530 \mathrm{E}-01$ & 4 & 6 & $5.631 \mathrm{E}-02$ & $1.914 \mathrm{E}+00$ & $3.757 E+07$ \\
\hline & 127976.340 & 166591.080 & $3.519 \mathrm{E}-01$ & 4 & 4 & $1.397 \mathrm{E}-02$ & $4.764 \mathrm{E}-01$ & $1.390 \mathrm{E}+07$ \\
\hline & 127825.080 & 166591.080 & $3.533 E-01$ & 2 & 4 & $7.014 \mathrm{E}-02$ & $1.191 \mathrm{E}+00$ & $3.516 \mathrm{E}+07$ \\
\hline \multirow{10}{*}{$\begin{array}{c}\mathrm{z}^{4} \mathrm{D}^{\circ} \rightarrow \mathrm{d}^{4} \mathrm{~F}^{\mathrm{e}} \\
*\end{array}$} & 0.5517 & 0.1303 & $4.214 \mathrm{E}-01$ & 20 & 28 & $1.899 \mathrm{E}-02$ & $2.704 \mathrm{E}+00$ & $1.935 \mathrm{E}+07$ \\
\hline & 128599.160 & 174529.270 & $4.185 E-01$ & 8 & 10 & $1.684 \mathrm{E}-02$ & $9.658 \mathrm{E}-01$ & $1.895 \mathrm{E}+07$ \\
\hline & & 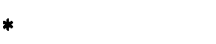 & & 8 & 8 & $1.938 \mathrm{E}-03$ & $1.101 \mathrm{E}-01$ & $2.778 \mathrm{E}+06$ \\
\hline & & * & & 8 & 6 & $9.690 \mathrm{E}-05$ & $5.602 \mathrm{E}-03$ & $1.789 \mathrm{E}+05$ \\
\hline & & * & & 6 & 8 & $1.550 \mathrm{E}-02$ & $6.626 \mathrm{E}-01$ & $1.657 \mathrm{E}+07$ \\
\hline & & $*$ & & 6 & 6 & $3.308 \mathrm{E}-03$ & $1.410 \mathrm{E}-01$ & $4.736 \mathrm{E}+06$ \\
\hline & & * & & 6 & 4 & $1.809 \mathrm{E}-04$ & $7.727 \mathrm{E}-03$ & $3.870 \mathrm{E}+05$ \\
\hline & & * & & 4 & 6 & $1.519 \mathrm{E}-02$ & $4.346 \mathrm{E}-01$ & $1.432 \mathrm{E}+07$ \\
\hline & & * & & 4 & 4 & $3.799 \mathrm{E}-03$ & $1.082 \mathrm{E}-01$ & $5.418 \mathrm{E}+06$ \\
\hline & & $*$ & & 2 & 4 & $1.899 \mathrm{E}-02$ & $2.704 \mathrm{E}-01$ & $1.354 \mathrm{E}+07$ \\
\hline \multirow[t]{9}{*}{$y^{4} D^{\circ} \rightarrow f^{4} P^{e}$} & 0.2878 & 0.2063 & $8.150 \mathrm{E}-02$ & 20 & 12 & $3.128 \mathrm{E}-01$ & $2.303 E+02$ & $2.782 \mathrm{E}+07$ \\
\hline & 157558.770 & 166479.820 & $8.130 \mathrm{E}-02$ & 8 & 6 & $3.121 \mathrm{E}-01$ & $9.212 \mathrm{E}+01$ & $2.209 \mathrm{E}+07$ \\
\hline & 157173.690 & 166479.820 & $8.480 \mathrm{E}-02$ & 6 & 6 & $9.765 \mathrm{E}-02$ & $2.073 \mathrm{E}+01$ & $5.640 \mathrm{E}+06$ \\
\hline & 156939.500 & 166479.820 & $8.700 \mathrm{E}-02$ & 4 & 6 & $1.670 \mathrm{E}-02$ & $2.303 E+00$ & $6.768 \mathrm{E}+05$ \\
\hline & 157173.690 & 165991.350 & $8.030 \mathrm{E}-02$ & 6 & 4 & $2.158 \mathrm{E}-01$ & $4.837 E+01$ & $1.676 \mathrm{E}+07$ \\
\hline & 156939.500 & 165991.350 & $8.250 \mathrm{E}-02$ & 4 & 4 & $1.689 \mathrm{E}-01$ & $2.457 \mathrm{E}+01$ & $9.233 E+06$ \\
\hline & 156829.750 & 165991.350 & $8.350 \mathrm{E}-02$ & 2 & 4 & $5.342 \mathrm{E}-02$ & $3.839 \mathrm{E}+00$ & $1.496 \mathrm{E}+06$ \\
\hline & 156939.500 & 165719.230 & $8.000 \mathrm{E}-02$ & 4 & 2 & $1.280 \mathrm{E}-01$ & $1.919 \mathrm{E}+01$ & $1.315 \mathrm{E}+07$ \\
\hline & 156829.750 & 165719.230 & $8.100 \mathrm{E}-02$ & 2 & 2 & $2.591 \mathrm{E}-01$ & $1.919 \mathrm{E}+01$ & $1.365 \mathrm{E}+07$ \\
\hline \multirow[t]{9}{*}{$\mathrm{y}^{4} \mathrm{D}^{\circ} \rightarrow \mathrm{g}^{4} \mathrm{P}^{\mathrm{e}}$} & 0.2878 & 0.1868 & $1.009 \mathrm{E}-01$ & 20 & 12 & $5.490 \mathrm{E}-03$ & $3.263 \mathrm{E}+00$ & $7.488 \mathrm{E}+05$ \\
\hline & 157558.770 & 168217.100 & $9.710 \mathrm{E}-02$ & 8 & 6 & $5.281 \mathrm{E}-03$ & $1.305 E+00$ & $5.333 \mathrm{E}+05$ \\
\hline & 157173.690 & 168217.100 & $1.006 \mathrm{E}-01$ & 6 & 6 & $1.642 \mathrm{E}-03$ & $2.937 \mathrm{E}-01$ & $1.334 \mathrm{E}+05$ \\
\hline & 156939.500 & 168217.100 & $1.028 \mathrm{E}-01$ & 4 & 6 & $2.796 \mathrm{E}-04$ & $3.263 \mathrm{E}-02$ & $1.582 \mathrm{E}+04$ \\
\hline & 157173.690 & 168397.890 & $1.023 \mathrm{E}-01$ & 6 & 4 & $3.895 \mathrm{E}-03$ & $6.853 \mathrm{E}-01$ & $4.911 \mathrm{E}+05$ \\
\hline & 156939.500 & 168397.890 & $1.045 \mathrm{E}-01$ & 4 & 4 & $3.031 \mathrm{E}-03$ & $3.481 \mathrm{E}-01$ & $2.659 \mathrm{E}+05$ \\
\hline & 156829.750 & 168397.890 & $1.055 \mathrm{E}-01$ & 2 & 4 & $9.564 \mathrm{E}-04$ & $5.439 \mathrm{E}-02$ & $4.275 \mathrm{E}+04$ \\
\hline & 156939.500 & 168490.430 & $1.053 \mathrm{E}-01$ & 4 & 2 & $2.386 \mathrm{E}-03$ & $2.720 \mathrm{E}-01$ & $4.251 E+05$ \\
\hline & 156829.750 & 168490.430 & $1.063 E-01$ & 2 & 2 & $4.818 \mathrm{E}-03$ & $2.720 \mathrm{E}-01$ & $4.373 E+05$ \\
\hline \multirow[t]{9}{*}{$\mathrm{y}^{4} \mathrm{D}^{\circ} \rightarrow \mathrm{h}^{4} \mathrm{P}^{*}$} & 0.2878 & 0.1369 & $1.509 \mathrm{E}-01$ & 20 & 12 & $4.008 \mathrm{E}-02$ & $1.594 \mathrm{E}+01$ & $1.221 \mathrm{E}+07$ \\
\hline & 157558.770 & 174098.100 & $1.507 \mathrm{E}-01$ & 8 & 6 & $4.004 \mathrm{E}-02$ & $6.377 \mathrm{E}+00$ & $9.739 E+06$ \\
\hline & 157173.690 & 174098.100 & $1.542 \mathrm{E}-01$ & 6 & 6 & $1.229 \mathrm{E}-02$ & $1.435 \mathrm{E}+\infty$ & $2.348 E+06$ \\
\hline & 156939.500 & 174098.100 & $1.564 \mathrm{E}-01$ & 4 & 6 & $2.078 \mathrm{E}-03$ & $1.594 \mathrm{E}-01$ & $2.722 \mathrm{E}+05$ \\
\hline & 157173.690 & 173595.770 & $1.496 \mathrm{E}-01$ & 6 & 4 & $2.782 \mathrm{E}-02$ & $3.348 \mathrm{E}+00$ & $7.503 E+06$ \\
\hline & 156939.500 & 173595.770 & $1.518 \mathrm{E}-01$ & 4 & 4 & $2.151 E-02$ & $1.701 \mathrm{E}+00$ & $3.982 E+06$ \\
\hline & 156829.750 & 173595.770 & $1.528 \mathrm{E}-01$ & 2 & 4 & $6.767 \mathrm{E}-03$ & $2.657 \mathrm{E}-01$ & $6.345 \mathrm{E}+05$ \\
\hline & 156939.500 & 173316.830 & $1.493 \mathrm{E}-01$ & 4 & 2 & $1.653 \mathrm{E}-02$ & $1.329 \mathrm{E}+00$ & $5.919 E+06$ \\
\hline & 156829.750 & 173316.830 & $1.503 E-01$ & 2 & 2 & $3.328 \mathrm{E}-02$ & $1.329 \mathrm{E}+00$ & $6.039 E+06$ \\
\hline \multirow[t]{9}{*}{$\mathrm{y}^{4} \mathrm{D}^{\circ} \rightarrow \mathrm{i}^{4} \mathrm{P}^{\bullet}$} & 0.2878 & 0.1263 & $1.615 \mathrm{E}-01$ & 20 & 12 & $1.206 \mathrm{E}-03$ & $4.482 \mathrm{E}-01$ & $4.211 E+05$ \\
\hline & 157558.770 & 174892.750 & $1.579 \mathrm{E}-01$ & 8 & 6 & $1.180 \mathrm{E}-03$ & $1.793 \mathrm{E}-01$ & $3.150 E+05$ \\
\hline & 157173.690 & 174892.750 & $1.614 \mathrm{E}-01$ & 6 & 6 & $3.617 \mathrm{E}-04$ & $4.034 \mathrm{E}-02$ & $7.568 \mathrm{E}+04$ \\
\hline & 156939.500 & 174892.750 & $1.636 \mathrm{E}-01$ & 4 & 6 & $6.111 \mathrm{E}-05$ & $4.482 \mathrm{E}-03$ & $8.758 \mathrm{E}+03$ \\
\hline & 157173.690 & 175019.780 & $1.626 \mathrm{E}-01$ & 6 & 4 & $8.503 \mathrm{E}-04$ & $9.412 \mathrm{E}-02$ & $2.708 \mathrm{E}+05$ \\
\hline & 156939.500 & 175019.780 & $1.648 \mathrm{E}-01$ & 4 & 4 & $6.566 \mathrm{E}-04$ & $4.781 \mathrm{E}-02$ & $1.432 \mathrm{E}+05$ \\
\hline & 156829.750 & 175019.780 & $1.658 \mathrm{E}-01$ & 2 & 4 & $2.064 \mathrm{E}-04$ & $7.470 \mathrm{E}-03$ & $2.279 \mathrm{E}+04$ \\
\hline & 156939.500 & 175083.510 & $1.654 \mathrm{E}-01$ & 4 & 2 & $5.148 \mathrm{E}-04$ & $3.735 E-02$ & $2.263 E+05$ \\
\hline & 156829.750 & 175083.510 & $1.664 \mathrm{E}-01$ & 2 & 2 & $1.036 \mathrm{E}-03$ & $3.735 \mathrm{E}-02$ & $2.304 \mathrm{E}+05$ \\
\hline
\end{tabular}


Table IV. Continued

\begin{tabular}{|c|c|c|c|c|c|c|c|c|}
\hline Transition & $\begin{array}{l}E_{i} \\
\mathrm{~cm}^{-1} \\
\end{array}$ & $\begin{array}{l}E_{f} \\
\mathrm{~cm}^{-1}\end{array}$ & $\begin{array}{l}E_{f i} \\
\mathrm{Ry}\end{array}$ & $g_{i}$ & $g_{f}$ & $f_{i f}$ & $S$ & $\begin{array}{l}A_{f i} \\
\mathrm{~s}^{-1} \\
\end{array}$ \\
\hline \multirow[t]{9}{*}{$y^{4} D^{\circ} \rightarrow j^{4} P^{e}$} & 0.2878 & 0.0905 & $1.972 \mathrm{E}-01$ & 20 & 12 & $4.921 \mathrm{E}-04$ & $1.497 \mathrm{E}-01$ & $2.563 \mathrm{E}+05$ \\
\hline & 157558.770 & 178848.960 & $1.940 \mathrm{E}-01$ & 8 & 6 & $4.840 \mathrm{E}-04$ & $5.988 \mathrm{E}-02$ & $1.951 \mathrm{E}+05$ \\
\hline & 157173.690 & 178848.960 & $1.975 \mathrm{E}-01$ & 6 & 6 & $1.478 \mathrm{E}-04$ & $1.347 \mathrm{E}-02$ & $4.631 \mathrm{E}+04$ \\
\hline & 156939.500 & 178848.960 & $1.997 \mathrm{E}-01$ & 4 & 6 & $2.491 \mathrm{E}-05$ & $1.497 \mathrm{E}-03$ & $5.320 \mathrm{E}+03$ \\
\hline & 157173.690 & 178915.200 & $1.981 \mathrm{E}-01$ & 6 & 4 & $3.460 \mathrm{E}-04$ & $3.144 \mathrm{E}-02$ & $1.636 \mathrm{E}+05$ \\
\hline & 156939.500 & 178915.200 & $2.003 E-01$ & 4 & 4 & $2.665 \mathrm{E}-04$ & $1.597 \mathrm{E}-02$ & $8.589 \mathrm{E}+04$ \\
\hline & 156829.750 & 178915.200 & $2.013 E-01$ & 2 & 4 & $8.370 E-05$ & $2.495 \mathrm{E}-03$ & $1.362 \mathrm{E}+04$ \\
\hline & 156939.500 & 178969.320 & $2.008 \mathrm{E}-01$ & 4 & 2 & $2.087 \mathrm{E}-04$ & $1.247 \mathrm{E}-02$ & $1.352 \mathrm{E}+05$ \\
\hline & 156829.750 & 178969.320 & $2.018 \mathrm{E}-01$ & 2 & 2 & $4.196 \mathrm{E}-04$ & $1.247 \mathrm{E}-02$ & $1.372 \mathrm{E}+05$ \\
\hline \multirow[t]{11}{*}{$\mathrm{y}^{4} \mathrm{D}^{\mathrm{a}} \rightarrow \mathrm{c}^{4} \mathrm{D}^{\mathrm{e}}$} & 0.2878 & 0.1950 & $9.274 \mathrm{E}-02$ & 20 & 20 & $1.850 \mathrm{E}-01$ & $1.197 \mathrm{E}+02$ & $1.278 \mathrm{E}+07$ \\
\hline & 157558.770 & 167575.230 & $9.130 \mathrm{E}-02$ & 8 & 8 & $1.561 \mathrm{E}-01$ & $4.105 \mathrm{E}+01$ & $1.045 \mathrm{E}+07$ \\
\hline & 157558.770 & 167364.980 & $8.930 \mathrm{E}-02$ & 8 & 6 & $2.538 \mathrm{E}-02$ & $6.821 \mathrm{E}+00$ & $2.168 E+06$ \\
\hline & 157173.690 & 167575.230 & $9.480 \mathrm{E}-02$ & 6 & 8 & $3.592 \mathrm{E}-02$ & $6.821 \mathrm{E}+\infty 0$ & $1.945 \mathrm{E}+06$ \\
\hline & 157173.690 & 167364.980 & $9.280 \mathrm{E}-02$ & 6 & 6 & $1.061 \mathrm{E}-01$ & $2.058 \mathrm{E}+01$ & $7.340 \mathrm{E}+06$ \\
\hline & 157173.690 & 167291.840 & $9.220 \mathrm{E}-02$ & 6 & 4 & $4.291 \mathrm{E}-02$ & $8.377 \mathrm{E}+\infty 0$ & $4.395 E+06$ \\
\hline & 156939.500 & 167364.980 & $9.500 \mathrm{E}-02$ & 4 & 6 & $6.632 \mathrm{E}-02$ & $8.377 \mathrm{E}+\infty 0$ & $3.205 \mathrm{E}+06$ \\
\hline & 156939.500 & 167291.840 & $9.440 \mathrm{E}-02$ & 4 & 4 & $7.531 \mathrm{E}-02$ & $9.573 \mathrm{E}+\infty 0$ & $5.391 \mathrm{E}+06$ \\
\hline & 156939.500 & 167254.840 & $9.400 \mathrm{E}-02$ & 4 & 2 & $4.687 \mathrm{E}-02$ & $5.983 \mathrm{E}+00$ & $6.653 E+06$ \\
\hline & 156829.750 & 167291.840 & $9.540 \mathrm{E}-02$ & 2 & 4 & $9.514 \mathrm{E}-02$ & $5.983 \mathrm{E}+\infty 0$ & $3.477 \mathrm{E}+06$ \\
\hline & 156829.750 & 167254.840 & $9.500 \mathrm{E}-02$ & 2 & 2 & $9.474 \mathrm{E}-02$ & $5.983 \mathrm{E}+\infty$ & $6.868 \mathrm{E}+06$ \\
\hline \multirow{11}{*}{$\begin{array}{c}y^{4} D^{\circ} \rightarrow d^{4} D^{6} \\
*\end{array}$} & 0.2878 & 0.1319 & $1.558 \mathrm{E}-01$ & 20 & 20 & $2.284 \mathrm{E}-02$ & $8.793 E+\infty 0$ & $4.455 \mathrm{E}+06$ \\
\hline & & & & 8 & 8 & $1.958 \mathrm{E}-02$ & $3.016 \mathrm{E}+00$ & $3.816 \mathrm{E}+06$ \\
\hline & 157558.770 & 174360.920 & $1.531 \mathrm{E}-01$ & 8 & 6 & $3.197 \mathrm{E}-03$ & $5.012 \mathrm{E}-01$ & $8.026 \mathrm{E}+05$ \\
\hline & & & & 6 & 8 & $4.350 \mathrm{E}-03$ & $5.012 \mathrm{E}-01$ & $6.397 \mathrm{E}+05$ \\
\hline & 157173.690 & 174360.920 & $1.566 \mathrm{E}-01$ & 6 & 6 & $1.316 \mathrm{E}-02$ & $1.512 \mathrm{E}+00$ & $2.592 \mathrm{E}+06$ \\
\hline & 157173.690 & 174329.310 & $1.563 \mathrm{E}-01$ & 6 & 4 & $5.345 \mathrm{E}-03$ & $6.155 \mathrm{E}-01$ & $1.573 \mathrm{E}+06$ \\
\hline & 156939.500 & 174360.920 & $1.588 \mathrm{E}-01$ & 4 & 6 & $8.145 E-03$ & $6.155 \mathrm{E}-01$ & $1.100 \mathrm{E}+06$ \\
\hline & 156939.500 & 174329.310 & $1.585 \mathrm{E}-01$ & 4 & 4 & $9.291 \mathrm{E}-03$ & $7.034 E-01$ & $1.875 \mathrm{E}+06$ \\
\hline & & & & 4 & 2 & $5.710 \mathrm{E}-03$ & $4.396 \mathrm{E}-01$ & $2.228 \mathrm{E}+06$ \\
\hline & 156829.750 & 174329.310 & $1.595 \mathrm{E}-01$ & 2 & 4 & $1.169 \mathrm{E}-02$ & $4.396 \mathrm{E}-01$ & $1.194 \mathrm{E}+06$ \\
\hline & & & & 2 & 2 & $1.142 \mathrm{E}-02$ & $4.396 \mathrm{E}-01$ & $2.228 \mathrm{E}+06$ \\
\hline \multirow{11}{*}{$\begin{array}{c}y^{4} D^{\circ} \rightarrow e^{4} D^{e} \\
*\end{array}$} & 0.2878 & 0.0955 & $1.923 \mathrm{E}-01$ & 20 & 20 & $7.468 \mathrm{E}-03$ & $2.331 \mathrm{E}+\infty$ & $2.217 \mathrm{E}+06$ \\
\hline & & & & 8 & 8 & $6.401 \mathrm{E}-03$ & $7.994 \mathrm{E}-01$ & $1.899 \mathrm{E}+06$ \\
\hline & 157558.770 & 178344.360 & $1.894 \mathrm{E}-01$ & 8 & 6 & $1.048 \mathrm{E}-03$ & $1.328 \mathrm{E}-01$ & $4.028 \mathrm{E}+05$ \\
\hline & & & & 6 & 8 & $1.422 \mathrm{E}-03$ & $1.328 \mathrm{E}-01$ & $3.183 \mathrm{E}+05$ \\
\hline & 157173.690 & 178344.360 & $1.929 \mathrm{E}-01$ & 6 & 6 & $4.296 \mathrm{E}-03$ & $4.009 \mathrm{E}-01$ & $1.284 \mathrm{E}+06$ \\
\hline & & & & 6 & 4 & $1.742 \mathrm{E}-03$ & $1.631 \mathrm{E}-01$ & $7.760 \mathrm{E}+05$ \\
\hline & 156939.500 & 178344.360 & $1.951 \mathrm{E}-01$ & 4 & 6 & $2.652 \mathrm{E}-03$ & $1.631 \mathrm{E}-01$ & $5.406 \mathrm{E}+05$ \\
\hline & & & & 4 & 4 & $2.987 \mathrm{E}-03$ & $1.864 \mathrm{E}-01$ & $8.869 \mathrm{E}+05$ \\
\hline & & & & 4 & 2 & $1.867 \mathrm{E}-03$ & $1.165 E-01$ & $1.109 \mathrm{E}+06$ \\
\hline & & & & 2 & 4 & $3.734 \mathrm{E}-03$ & $1.165 \mathrm{E}-01$ & $5.543 \mathrm{E}+05$ \\
\hline & & & & 2 & 2 & $3.734 \mathrm{E}-03$ & $1.165 \mathrm{E}-01$ & $1.109 \mathrm{E}+06$ \\
\hline \multirow[t]{10}{*}{$y^{4} D^{\circ} \rightarrow c^{4} F^{e}$} & 0.2878 & 0.1993 & $8.844 \mathrm{E}-02$ & 20 & 28 & $1.070 \mathrm{E}+00$ & $7.259 \mathrm{E}+02$ & $4.802 \mathrm{E}+07$ \\
\hline & 157558.770 & 167269.150 & $8.850 \mathrm{E}-02$ & 8 & 10 & $9.560 \mathrm{E}-01$ & $2.593 \mathrm{E}+02$ & $4.811 \mathrm{E}+07$ \\
\hline & 157558.770 & 166918.270 & $8.530 \mathrm{E}-02$ & 8 & 8 & $1.050 \mathrm{E}-01$ & $2.956 \mathrm{E}+01$ & $6.139 \mathrm{E}+06$ \\
\hline & 157558.770 & 166709.270 & $8.340 \mathrm{E}-02$ & 8 & 6 & $5.225 \mathrm{E}-03$ & $1.504 \mathrm{E}+00$ & $3.892 \mathrm{E}+05$ \\
\hline & 157173.690 & 166918.270 & $8.880 E-02$ & 6 & 8 & $8.774 \mathrm{E}-01$ & $1.778 \mathrm{E}+02$ & $4.168 \mathrm{E}+07$ \\
\hline & 157173.690 & 166709.270 & $8.690 \mathrm{E}-02$ & 6 & 6 & $1.827 \mathrm{E}-01$ & $3.785 \mathrm{E}+01$ & $1.108 \mathrm{E}+07$ \\
\hline & 157173.690 & 166591.080 & $8.580 E-02$ & 6 & 4 & $9.886 \mathrm{E}-03$ & $2.074 \mathrm{E}+\infty 0$ & $8.769 \mathrm{E}+05$ \\
\hline & 156939.500 & 166709.270 & $8.910 \mathrm{E}-02$ & 4 & 6 & $8.662 \mathrm{E}-01$ & $1.167 \mathrm{E}+02$ & $3.682 \mathrm{E}+07$ \\
\hline & 156939.500 & 166591.080 & $8.800 E-02$ & 4 & 4 & $2.129 \mathrm{E}-01$ & $2.904 \mathrm{E}+01$ & $1.324 \mathrm{E}+07$ \\
\hline & 156829.750 & 166591.080 & $8.900 \mathrm{E}-02$ & 2 & 4 & $1.077 \mathrm{E}+00$ & $7.259 \mathrm{E}+01$ & $3.425 \mathrm{E}+07$ \\
\hline \multirow{10}{*}{$\begin{array}{c}y^{4} D^{\circ} \rightarrow d^{4} F^{e} \\
*\end{array}$} & 0.2878 & 0.1303 & $1.575 \mathrm{E}-01$ & 20 & 28 & $1.168 \mathrm{E}-01$ & $4.448 \mathrm{E}+01$ & $1.662 \mathrm{E}+07$ \\
\hline & 157558.770 & 174529.270 & $1.546 \mathrm{E}-01$ & 8 & 10 & $1.023 \mathrm{E}-01$ & $1.589 \mathrm{E}+01$ & $1.572 \mathrm{E}+07$ \\
\hline & & & & 8 & 8 & $1.191 \mathrm{E}-02$ & $1.811 \mathrm{E}+\infty$ & $2.386 \mathrm{E}+06$ \\
\hline & & & & 8 & 6 & $5.957 \mathrm{E}-04$ & $9.214 \mathrm{E}-02$ & $1.536 \mathrm{E}+05$ \\
\hline & & & & 6 & 8 & $9.531 \mathrm{E}-02$ & $1.090 \mathrm{E}+01$ & $1.423 \mathrm{E}+07$ \\
\hline & & & & 6 & 6 & $2.033 \mathrm{E}-02$ & $2.319 \mathrm{E}+\infty 0$ & $4.067 \mathrm{E}+06$ \\
\hline & & & & 6 & 4 & $1.112 \mathrm{E}-03$ & $1.271 \mathrm{E}-01$ & $3.323 E+05$ \\
\hline & & & & 4 & 6 & $9.340 \mathrm{E}-02$ & $7.149 \mathrm{E}+\infty$ & $1.230 \mathrm{E}+07$ \\
\hline & & & & 4 & 4 & $2.335 \mathrm{E}-02$ & $1.779 \mathrm{E}+\infty 0$ & $4.652 \mathrm{E}+06$ \\
\hline & & & & 2 & 4 & $1.168 \mathrm{E}-01$ & $4.448 \mathrm{E}+00$ & $1.163 \mathrm{E}+07$ \\
\hline
\end{tabular}


Table IV. Continued

\begin{tabular}{|c|c|c|c|c|c|c|c|c|}
\hline Transition & $\begin{array}{l}E_{i} \\
\mathrm{~cm}^{-1}\end{array}$ & $\begin{array}{l}E_{f} \\
\mathrm{~cm}^{-1}\end{array}$ & $\begin{array}{l}E_{f i} \\
\mathrm{Ry}\end{array}$ & $g_{i}$ & $g_{f}$ & $f_{i f}$ & $S$ & $\begin{array}{l}A_{f i} \\
\mathrm{~s}^{-1}\end{array}$ \\
\hline \multirow{3}{*}{$\mathrm{a}^{2} \mathrm{~S}^{\mathrm{e}} \rightarrow \mathrm{y}^{2} \mathrm{P}^{\mathrm{o}}$} & 0.6291 & 0.5055 & $1.237 \mathrm{E}-01$ & 2 & 6 & $2.529 \mathrm{E}-03$ & $1.227 \mathrm{E}-01$ & $1.036 \mathrm{E}+05$ \\
\hline & 119783.770 & 133399.970 & $1.240 \mathrm{E}-01$ & 2 & 4 & $1.690 \mathrm{E}-03$ & $8.178 \mathrm{E}-02$ & $1.044 \mathrm{E}+05$ \\
\hline & 119783.770 & 133268.680 & $1.228 \mathrm{E}-01$ & 2 & 2 & $8.369 E-04$ & $4.089 \mathrm{E}-02$ & $1.014 \mathrm{E}+05$ \\
\hline \multirow[t]{3}{*}{$\mathrm{a}^{2} \mathrm{~S}^{\mathrm{e}} \rightarrow \mathrm{x}^{2} \mathrm{P}^{\circ}$} & 0.6291 & 0.4123 & $2.168 \mathrm{E}-01$ & 2 & 6 & $4.501 \mathrm{E}-02$ & $1.245 \mathrm{E}+00$ & $5.666 \mathrm{E}+06$ \\
\hline & 119783.770 & 143623.560 & $2.172 \mathrm{E}-01$ & 2 & 4 & $3.006 \mathrm{E}-02$ & $8.303 E-01$ & $5.694 \mathrm{E}+06$ \\
\hline & 119783.770 & 143488.950 & $2.160 \mathrm{E}-01$ & 2 & 2 & $1.494 \mathrm{E}-02$ & $4.151 \mathrm{E}-01$ & $5.601 \mathrm{E}+06$ \\
\hline \multirow{3}{*}{$a^{2} S^{e} \rightarrow w^{2} P^{\circ}$} & 0.6291 & 0.2969 & $3.322 \mathrm{E}-01$ & 2 & 6 & $2.308 \mathrm{E}-03$ & $4.168 \mathrm{E}-02$ & $6.819 \mathrm{E}+05$ \\
\hline & 119783.770 & 156276.830 & $3.325 \mathrm{E}-01$ & 2 & 4 & $1.540 \mathrm{E}-03$ & $2.778 \mathrm{E}-02$ & $6.836 \mathrm{E}+05$ \\
\hline & 119783.770 & 156167.040 & $3.315 \mathrm{E}-01$ & 2 & 2 & $7.675 \mathrm{E}-04$ & $1.389 \mathrm{E}-02$ & $6.775 \mathrm{E}+05$ \\
\hline \multirow{3}{*}{$\mathrm{b}^{2} \mathrm{~S}^{e} \rightarrow \mathrm{x}^{2} \mathrm{P}^{\circ}$} & 0.4784 & 0.4123 & $6.607 \mathrm{E}-02$ & 2 & 6 & $1.992 \mathrm{E}-02$ & $1.809 \mathrm{E}+00$ & $2.328 \mathrm{E}+05$ \\
\hline & 136328.790 & 143623.560 & $6.650 \mathrm{E}-02$ & 2 & 4 & $1.337 \mathrm{E}-02$ & $1.206 \mathrm{E}+00$ & $2.374 \mathrm{E}+05$ \\
\hline & 136328.790 & 143488.950 & $6.530 \mathrm{E}-02$ & 2 & 2 & $6.563 \mathrm{E}-03$ & $6.030 \mathrm{E}-01$ & $2.248 \mathrm{E}+05$ \\
\hline \multirow[t]{3}{*}{$b^{2} S^{\circ} \rightarrow w^{2} P^{\circ}$} & 0.4784 & 0.2969 & $1.814 \mathrm{E}-1$ & 2 & 6 & $8.580 \mathrm{E}-01$ & $2.837 \mathrm{E}+01$ & $7.563 \mathrm{E}+07$ \\
\hline & 136328.790 & 156276.830 & $1.818 \mathrm{E}-01$ & 2 & 4 & $5.731 \mathrm{E}-01$ & $1.891 \mathrm{E}+01$ & $7.607 \mathrm{E}+07$ \\
\hline & 136328.790 & 156167.040 & $1.808 \mathrm{E}-01$ & 2 & 2 & $2.850 \mathrm{E}-01$ & $9.457 \mathrm{E}+00$ & $7.483 \mathrm{E}+07$ \\
\hline \multirow{3}{*}{$\mathrm{c}^{2} \mathrm{~S}^{\mathrm{e}} \rightarrow \mathrm{w}^{2} \mathrm{P}^{\mathrm{o}}$} & 0.3387 & 0.2969 & $4.181 \mathrm{E}-02$ & 2 & 6 & $2.496 \mathrm{E}-02$ & $3.582 \mathrm{E}+\infty$ & $1.168 E+05$ \\
\hline & 151651.720 & 156276.830 & $4.210 \mathrm{E}-02$ & 2 & 4 & $1.675 \mathrm{E}-02$ & $2.388 \mathrm{E}+00$ & $1.193 \mathrm{E}+05$ \\
\hline & 151651.720 & 156167.040 & $4.110 \mathrm{E}-02$ & 2 & 2 & $8.178 \mathrm{E}-03$ & $1.194 \mathrm{E}+\infty$ & $1.110 E+05$ \\
\hline \multirow[t]{3}{*}{$a^{2} P^{e} \rightarrow z^{2} S^{o}$} & 0.7571 & 0.5772 & $1.799 \mathrm{E}-01$ & 6 & 2 & $4.495 \mathrm{E}-02$ & $4.498 \mathrm{E}+\infty 0$ & $3.504 \mathrm{E}+07$ \\
\hline & 105599.060 & 125485.290 & $1.812 \mathrm{E}-01$ & 4 & 2 & $4.529 \mathrm{E}-02$ & $2.999 \mathrm{E}+\infty 0$ & $2.389 E+07$ \\
\hline & 106044.240 & 125485.290 & $1.772 \mathrm{E}-01$ & 2 & 2 & $4.427 \mathrm{E}-02$ & $1.499 \mathrm{E}+\infty 0$ & $1.116 \mathrm{E}+07$ \\
\hline \multirow[t]{5}{*}{$\mathrm{a}^{2} \mathrm{P}^{\mathrm{e}} \rightarrow \mathrm{y}^{2} \mathrm{P}^{\mathrm{o}}$} & 0.7571 & 0.5055 & $2.516 \mathrm{E}-01$ & 6 & 6 & $4.885 \mathrm{E}-02$ & $3.495 \mathrm{E}+\infty 0$ & $2.484 \mathrm{E}+07$ \\
\hline & 105599.060 & 133399.970 & $2.533 \mathrm{E}-01$ & 4 & 4 & $4.099 \mathrm{E}-02$ & $1.942 \mathrm{E}+\infty 0$ & $2.113 \mathrm{E}+07$ \\
\hline & 105599.060 & 133268.680 & $2.521 \mathrm{E}-01$ & 4 & 2 & $8.159 \mathrm{E}-03$ & $3.884 \mathrm{E}-01$ & $8.331 E+06$ \\
\hline & 106044.240 & 133399.970 & $2.493 \mathrm{E}-01$ & 2 & 4 & $1.613 \mathrm{E}-02$ & $3.884 \mathrm{E}-01$ & $4.025 \mathrm{E}+06$ \\
\hline & 106044.240 & 133268.680 & $2.480 \mathrm{E}-01$ & 2 & 2 & $3.211 \mathrm{E}-02$ & $7.767 \mathrm{E}-01$ & $1.587 \mathrm{E}+07$ \\
\hline \multirow{5}{*}{$\mathrm{a}^{2} \mathrm{P}^{\mathrm{B}} \rightarrow \mathrm{x}^{2} \mathrm{P}^{\circ}$} & 0.7571 & 0.4123 & $3.447 \mathrm{E}-01$ & 6 & 6 & $1.604 \mathrm{E}-03$ & $8.376 \mathrm{E}-02$ & $1.531 \mathrm{E}+06$ \\
\hline & 105599.060 & 143623.560 & $3.465 \mathrm{E}-01$ & 4 & 4 & $1.344 \mathrm{E}-03$ & $4.654 \mathrm{E}-02$ & $1.296 \mathrm{E}+06$ \\
\hline & 105599.060 & 143488.950 & $3.453 \mathrm{E}-01$ & 4 & 2 & $2.678 \mathrm{E}-04$ & $9.307 \mathrm{E}-03$ & $5.130 \mathrm{E}+05$ \\
\hline & 106044.240 & 143623.560 & $3.424 \mathrm{E}-01$ & 2 & 4 & $5.312 \mathrm{E}-04$ & $9.307 \mathrm{E}-03$ & $2.502 \mathrm{E}+05$ \\
\hline & 106044.240 & 143488.950 & $3.413 \mathrm{E}-01$ & 2 & 2 & $1.059 \mathrm{E}-03$ & $1.861 \mathrm{E}-02$ & $9.903 \mathrm{E}+05$ \\
\hline \multirow[t]{5}{*}{$\mathrm{a}^{2} \mathrm{P}^{\mathrm{e}} \rightarrow \mathrm{w}^{2} \mathrm{P}^{\circ}$} & 0.7571 & 0.2969 & $4.601 \mathrm{E}-01$ & 6 & 6 & $2.065 \mathrm{E}-04$ & $8.080 \mathrm{E}-03$ & $3.512 \mathrm{E}+05$ \\
\hline & 105599.060 & 156276.830 & $4.618 \mathrm{E}-01$ & 4 & 4 & $1.727 \mathrm{E}-04$ & $4.489 \mathrm{E}-03$ & $2.959 \mathrm{E}+05$ \\
\hline & 105599.060 & 156167.040 & $4.608 \mathrm{E}-01$ & 4 & 2 & $3.447 \mathrm{E}-05$ & $8.977 \mathrm{E}-0.4$ & $1.176 \mathrm{E}+05$ \\
\hline & 106044.240 & 156276.830 & $4.578 \mathrm{E}-01$ & 2 & 4 & $6.849 E-05$ & $8.977 \mathrm{E}-04$ & $5.764 \mathrm{E}+04$ \\
\hline & 106044.240 & 156167.040 & $4.567 \mathrm{E}-01$ & 2 & 2 & $1.367 \mathrm{E}-04$ & $1.795 \mathrm{E}-03$ & $2.290 \mathrm{E}+05$ \\
\hline \multirow[t]{4}{*}{$a^{2} P^{e} \rightarrow y^{2} D^{o}$} & 0.7571 & 0.5272 & $2.298 \mathrm{E}-01$ & 6 & 10 & $3.050 \mathrm{E}-03$ & $2.389 \mathrm{E}-01$ & $7.764 \mathrm{E}+05$ \\
\hline & 105599.060 & 131187.190 & $2.332 \mathrm{E}-01$ & 4 & 6 & $2.785 \mathrm{E}-03$ & $1.433 \mathrm{E}-01$ & $8.111 E+05$ \\
\hline & 105599.060 & 130641.110 & $2.282 \mathrm{E}-01$ & 4 & 4 & $3.028 \mathrm{E}-04$ & $1.592 \mathrm{E}-02$ & $1.267 \mathrm{E}+05$ \\
\hline & 106044.240 & 130641.110 & $2.242 \mathrm{E}-01$ & 2 & 4 & $2.974 \mathrm{E}-03$ & $7.962 \mathrm{E}-02$ & $6.002 \mathrm{E}+05$ \\
\hline \multirow[t]{4}{*}{$a^{2} P^{e} \rightarrow x^{2} D^{\circ}$} & 0.7571 & 0.4383 & $3.187 E-01$ & 6 & 10 & $6.201 \mathrm{E}-03$ & $3.502 \mathrm{E}-01$ & $3.036 \mathrm{E}+06$ \\
\hline & 105599.060 & 140708.890 & $3.199 \mathrm{E}-01$ & 4 & 6 & $5.601 \mathrm{E}-03$ & $2.101 \mathrm{E}-01$ & $3.070 \mathrm{E}+06$ \\
\hline & 105599.060 & 140750.340 & $3.203 \mathrm{E}-01$ & 4 & 4 & $6.231 \mathrm{E}-04$ & $2.334 \mathrm{E}-02$ & $5.135 \mathrm{E}+05$ \\
\hline & 106044.240 & 140750.340 & $3.163 E-01$ & 2 & 4 & $6.152 E-03$ & $1.167 \mathrm{E}-01$ & $2.471 \mathrm{E}+06$ \\
\hline \multirow[t]{4}{*}{$a^{2} P^{\circ} \rightarrow w^{2} D^{\circ}$} & 0.7571 & 0.2762 & $4.809 \mathrm{E}-01$ & 6 & 10 & $8.322 E-03$ & $3.115 \mathrm{E}-01$ & $9.274 \mathrm{E}+06$ \\
\hline & 105599.060 & 158715.460 & $4.840 \mathrm{E}-01$ & 4 & 6 & $7.539 \mathrm{E}-03$ & $1.869 \mathrm{E}-01$ & $9.457 \mathrm{E}+06$ \\
\hline & 105599.060 & 158215.590 & $4.795 \mathrm{E}-01$ & 4 & 4 & $8.299 \mathrm{E}-04$ & $2.077 \mathrm{E}-02$ & $1.533 \mathrm{E}+06$ \\
\hline & 106044.240 & 158215.590 & $4.755 \mathrm{E}-01$ & 2 & 4 & $8.229 \mathrm{E}-03$ & $1.038 \mathrm{E}-01$ & $7.470 \mathrm{E}+06$ \\
\hline
\end{tabular}


Table IV. Continued

\begin{tabular}{|c|c|c|c|c|c|c|c|c|}
\hline Transition & $\begin{array}{l}E_{i} \\
\mathrm{~cm}^{-1}\end{array}$ & $\begin{array}{l}E_{f} \\
\mathrm{~cm}^{-1} \\
\end{array}$ & $\begin{array}{l}E_{f i} \\
\text { Ry }\end{array}$ & $g_{i}$ & $g_{f}$ & $f_{i j}$ & $S$ & $\begin{array}{l}A_{f i} \\
\mathrm{~s}^{-1} \\
\end{array}$ \\
\hline \multirow[t]{3}{*}{$\mathrm{b}^{2} \mathrm{P}^{\mathbf{e}} \rightarrow \mathrm{z}^{2} \mathrm{~S}^{\mathbf{0}}$} & 0.6884 & 0.5772 & $1.112 \mathrm{E}-01$ & 6 & 2 & $8.862 \mathrm{E}-02$ & $1.435 \mathrm{E}+01$ & $2.639 \mathrm{E}+07$ \\
\hline & 113461.540 & 125485.290 & $1.096 \mathrm{E}-01$ & 4 & 2 & $8.738 \mathrm{E}-02$ & $9.567 \mathrm{E}+00$ & $1.686 \mathrm{E}+07$ \\
\hline & 112937.570 & 125485.290 & $1.143 \mathrm{E}-01$ & 2 & 2 & $9.112 \mathrm{E}-02$ & $4.783 \mathrm{E}+00$ & $9.562 \mathrm{E}+06$ \\
\hline \multirow[t]{5}{*}{$b^{2} P^{e} \rightarrow y^{2} P^{o}$} & 0.6884 & 0.5055 & $1.829 \mathrm{E}-01$ & 6 & 6 & $3.145 \mathrm{E}-01$ & $3.095 \mathrm{E}+01$ & $8.448 E+07$ \\
\hline & 113461.540 & 133399.970 & $1.817 \mathrm{E}-01$ & 4 & 4 & $2.604 \mathrm{E}-01$ & $1.719 E+01$ & $6.904 \mathrm{E}+07$ \\
\hline & 113461.540 & 133268.680 & $1.805 \mathrm{E}-01$ & 4 & 2 & $5.173 \mathrm{E}-02$ & $3.439 E+00$ & $2.707 \mathrm{E}+07$ \\
\hline & 112937.570 & 133399.970 & $1.864 \mathrm{E}-01$ & 2 & 4 & $1.068 \mathrm{E}-01$ & $3.439 E+00$ & $1.491 \mathrm{E}+07$ \\
\hline & 112937.570 & 133268.680 & $1.852 \mathrm{E}-01$ & 2 & 2 & $2.123 \mathrm{E}-01$ & $6.878 \mathrm{E}+00$ & $5.849 E+07$ \\
\hline \multirow[t]{5}{*}{$b^{2} P^{e} \rightarrow x^{2} P^{o}$} & 0.6884 & 0.4123 & $2.760 \mathrm{E}-01$ & 6 & 6 & $3.244 \mathrm{E}-02$ & $2.115 E+\infty 0$ & $1.985 \mathrm{E}+07$ \\
\hline & 113461.540 & 143623.560 & $2.749 \mathrm{E}-01$ & 4 & 4 & $2.692 \mathrm{E}-02$ & $1.175 E+\infty 0$ & $1.634 \mathrm{E}+07$ \\
\hline & 113461.540 & 143488.950 & $2.737 \mathrm{E}-01$ & 4 & 2 & $5.361 \mathrm{E}-03$ & $2.350 \mathrm{E}-01$ & $6.451 \mathrm{E}+06$ \\
\hline & 112937.570 & 143623.560 & $2.796 \mathrm{E}-01$ & 2 & 4 & $1.095 \mathrm{E}-02$ & $2.350 \mathrm{E}-01$ & $3.439 E+06$ \\
\hline & 112937.570 & 143488.950 & $2.784 \mathrm{E}-01$ & 2 & 2 & $2.181 \mathrm{E}-02$ & $4.701 \mathrm{E}-01$ & $1.358 \mathrm{E}+07$ \\
\hline \multirow[t]{5}{*}{$b^{2} P^{e} \rightarrow w^{2} P^{o}$} & 0.6884 & 0.2969 & $3.914 \mathrm{E}-01$ & 6 & 6 & $9.052 \mathrm{E}-04$ & $4.162 \mathrm{E}-02$ & $1.114 \mathrm{E}+06$ \\
\hline & 113461.540 & 156276.830 & $3.902 \mathrm{E}-01$ & 4 & 4 & $7.519 E-04$ & $2.312 \mathrm{E}-02$ & $9.196 \mathrm{E}+05$ \\
\hline & 113461.540 & 156167.040 & $3.892 \mathrm{E}-01$ & 4 & 2 & $1.500 \mathrm{E}-04$ & $4.625 \mathrm{E}-03$ & $3.650 \mathrm{E}+05$ \\
\hline & 112937.570 & 156276.830 & $3.949 \mathrm{E}-01$ & 2 & 4 & $3.044 \mathrm{E}-04$ & $4.625 \mathrm{E}-03$ & $1.906 \mathrm{E}+05$ \\
\hline & 112937.570 & 156167.040 & $3.939 \mathrm{E}-01$ & 2 & 2 & $6.073 E-04$ & $9.250 \mathrm{E}-03$ & $7.568 \mathrm{E}+05$ \\
\hline \multirow[t]{4}{*}{$\mathrm{b}^{2} \mathrm{P}^{\mathrm{e}} \rightarrow \mathrm{y}^{2} \mathrm{D}^{\mathrm{o}}$} & 0.6884 & 0.5272 & $1.611 \mathrm{E}-01$ & 6 & 10 & $5.073 \mathrm{E}-01$ & $5.667 \mathrm{E}+01$ & $6.348 \mathrm{E}+07$ \\
\hline & 113461.540 & 131187.190 & $1.616 \mathrm{E}-01$ & 4 & 6 & $4.579 \mathrm{E}-01$ & $3.400 \mathrm{E}+01$ & $6.404 \mathrm{E}+07$ \\
\hline & 113461.540 & 130641.110 & $1.566 \mathrm{E}-01$ & 4 & 4 & $4.931 \mathrm{E}-02$ & $3.778 \mathrm{E}+00$ & $9.712 \mathrm{E}+06$ \\
\hline & 112937.570 & 130641.110 & $1.613 \mathrm{E}-01$ & 2 & 4 & $5.079 \mathrm{E}-01$ & $1.889 \mathrm{E}+01$ & $5.307 \mathrm{E}+07$ \\
\hline \multirow[t]{4}{*}{$b^{2} P^{e} \rightarrow x^{2} D^{o}$} & 0.6884 & 0.4383 & $2.500 \mathrm{E}-01$ & 6 & 10 & $7.961 \mathrm{E}-02$ & $5.731 \mathrm{E}+00$ & $2.399 \mathrm{E}+07$ \\
\hline & 113461.540 & 140708.890 & $2.483 \mathrm{E}-01$ & 4 & 6 & $7.115 \mathrm{E}-02$ & $3.439 E+00$ & $2.349 \mathrm{E}+07$ \\
\hline & 113461.540 & 140750.340 & $2.487 \mathrm{E}-01$ & 4 & 4 & $7.919 \mathrm{E}-03$ & $3.821 \mathrm{E}-01$ & $3.934 E+06$ \\
\hline & 112937.570 & 140750.340 & $2.534 \mathrm{E}-01$ & 2 & 4 & $8.068 \mathrm{E}-02$ & $1.910 \mathrm{E}+00$ & $2.081 \mathrm{E}+07$ \\
\hline \multirow[t]{4}{*}{$b^{2} P^{e} \rightarrow w^{2} D^{\circ}$} & 0.6884 & 0.2762 & $4.122 \mathrm{E}-01$ & 6 & 10 & $5.218 \mathrm{E}-03$ & $2.279 \mathrm{E}-01$ & $4.272 \mathrm{E}+06$ \\
\hline & 113461.540 & 158715.460 & $4.124 \mathrm{E}-01$ & 4 & 6 & $4.699 \mathrm{E}-03$ & $1.367 \mathrm{E}-01$ & $4.280 \mathrm{E}+06$ \\
\hline & 113461.540 & 158215.590 & $4.079 \mathrm{E}-01$ & 4 & 4 & $5.164 \mathrm{E}-04$ & $1.519 \mathrm{E}-02$ & $6.902 \mathrm{E}+05$ \\
\hline & 112937.570 & 158215.590 & $4.126 \mathrm{E}-01$ & 2 & 4 & $5.224 \mathrm{E}-03$ & $7.597 \mathrm{E}-02$ & $3.572 \mathrm{E}+06$ \\
\hline \multirow[t]{5}{*}{$c^{2} P^{\circ} \rightarrow x^{2} P^{\circ}$} & 0.4453 & 0.4123 & $3.298 \mathrm{E}-02$ & 6 & 6 & $5.202 \mathrm{E}-02$ & $2.839 \mathrm{E}+01$ & $4.545 \mathrm{E}+05$ \\
\hline & 140016.770 & 143623.560 & $3.290 \mathrm{E}-02$ & 4 & 4 & $4.324 \mathrm{E}-02$ & $1.577 \mathrm{E}+01$ & $3.760 \mathrm{E}+05$ \\
\hline & 140016.770 & 143488.950 & $3.170 \mathrm{E}-02$ & 4 & 2 & $8.333 \mathrm{E} 03$ & $3.154 E+00$ & $1.345 \mathrm{E}+05$ \\
\hline & 139844.990 & 143623.560 & $3.440 \mathrm{E}-02$ & 2 & 4 & $1.809 \mathrm{E}-02$ & $3.154 \mathrm{E}+00$ & $8.595 \mathrm{E}+04$ \\
\hline & 139844.990 & 143488.950 & $3.320 \mathrm{E}-02$ & 2 & 2 & $3.491 E-02$ & $6.309 E+00$ & $3.091 \mathrm{E}+05$ \\
\hline \multirow[t]{5}{*}{$c^{2} P^{c} \rightarrow w^{2} P^{o}$} & 0.4453 & 0.2969 & $1.484 \mathrm{E}-01$ & 6 & 6 & $1.217 \mathrm{E}-03$ & $1.477 \mathrm{E}-01$ & $2.152 \mathrm{E}+05$ \\
\hline & 140016.770 & 156276.830 & $1.482 \mathrm{E}-01$ & 4 & 4 & $1.013 E-03$ & $8.203 \mathrm{E}-02$ & $1.787 \mathrm{E}+05$ \\
\hline & 140016.770 & 156167.040 & $1.472 \mathrm{E}-01$ & 4 & 2 & $2.013 E-04$ & $1.641 \mathrm{E}-02$ & $7.005 \mathrm{E}+04$ \\
\hline & 139844.990 & 156276.830 & $1.497 \mathrm{E}-01$ & 2 & 4 & $4.093 \mathrm{E}-04$ & $1.641 \mathrm{E}-02$ & $3.684 \mathrm{E}+04$ \\
\hline & 139844.990 & 156167.040 & $1.487 \mathrm{E}-01$ & 2 & 2 & $8.132 E-04$ & $3.281 \mathrm{E}-02$ & $1.444 \mathrm{E}+05$ \\
\hline \multirow[t]{4}{*}{$c^{2} P^{e} \rightarrow x^{2} D^{\circ}$} & 0.4453 & 0.4383 & $6.980 \mathrm{E}-03$ & 6 & 10 & $2.004 \mathrm{E}-03$ & $5.167 \mathrm{E}+00$ & $4.705 \mathrm{E}+02$ \\
\hline & 140016.770 & 140708.890 & $6.300 \mathrm{E}-03$ & 4 & 6 & $1.628 \mathrm{E}-03$ & $3.100 \mathrm{E}+00$ & $3.460 \mathrm{E}+02$ \\
\hline & 140016.770 & 140750.340 & $6.700 \mathrm{E}-03$ & 4 & 4 & $1.923 \mathrm{E}-04$ & $3.445 \mathrm{E}-01$ & $6.935 \mathrm{E}+01$ \\
\hline & 139844.990 & 140750.340 & $8.200 \mathrm{E}-03$ & 2 & 4 & $2.354 \mathrm{E}-03$ & $1.722 \mathrm{E}+00$ & $6.357 \mathrm{E}+02$ \\
\hline \multirow[t]{4}{*}{$c^{2} P^{\circ} \rightarrow w^{2} D^{\circ}$} & 0.4453 & 0.2762 & $1.691 \mathrm{E}-01$ & 6 & 10 & $1.203 \mathrm{E}-02$ & $1.280 \mathrm{E}+00$ & $1.657 \mathrm{E}+06$ \\
\hline & 140016.770 & 158715.460 & $1.704 \mathrm{E}-01$ & 4 & 6 & $1.091 \mathrm{E}-02$ & $7.681 \mathrm{E}-01$ & $1.696 \mathrm{E}+06$ \\
\hline & 140016.770 & 158215.590 & $1.659 \mathrm{E}-01$ & 4 & 4 & $1.180 \mathrm{E}-03$ & $8.534 \mathrm{E}-02$ & $2.608 \mathrm{E}+05$ \\
\hline & 139844.990 & 158215.590 & $1.674 \mathrm{E}-01$ & 2 & 4 & $1.190 \mathrm{E}-02$ & $4.267 \mathrm{E}-01$ & $1.340 \mathrm{E}+06$ \\
\hline
\end{tabular}


Table IV. Continued

\begin{tabular}{|c|c|c|c|c|c|c|c|c|}
\hline Transition & $\begin{array}{l}E_{i} \\
\mathrm{~cm}^{-1}\end{array}$ & $\begin{array}{l}E_{f} \\
\mathrm{~cm}^{-1}\end{array}$ & $\begin{array}{l}E_{f i} \\
R y\end{array}$ & $g_{i}$ & $g_{f}$ & $f_{i f}$ & $S$ & $\begin{array}{l}A_{f i} \\
\mathrm{~s}^{-1} \\
\end{array}$ \\
\hline \multirow{5}{*}{$\mathrm{d}^{2} \mathrm{P}^{\mathrm{e}} \rightarrow \mathrm{w}^{2} \mathrm{P}^{\mathrm{o}}$} & 0.3936 & 0.2969 & $9.669 \mathrm{E}-02$ & 6 & 6 & $2.182 \mathrm{E}-02$ & $4.062 \mathrm{E}+00$ & $1.638 \mathrm{E}+06$ \\
\hline & 145505.740 & 156276.830 & $9.820 \mathrm{E}-02$ & 4 & 4 & $1.847 \mathrm{E}-02$ & $2.257 \mathrm{E}+00$ & $1.430 \mathrm{E}+06$ \\
\hline & 145505.740 & 156167.040 & $9.720 \mathrm{E}-02$ & 4 & 2 & $3.656 \mathrm{E}-03$ & $4.513 \mathrm{E}-01$ & $5.548 \mathrm{E}+05$ \\
\hline & 145877.660 & 156276.830 & $9.480 \mathrm{E}-02$ & 2 & 4 & $7.131 \mathrm{E}-03$ & $4.513 \mathrm{E}-01$ & $2.574 \mathrm{E}+05$ \\
\hline & 145877.660 & 156167.040 & $9.380 \mathrm{E}-02$ & 2 & 2 & $1.411 \mathrm{E}-02$ & $9.026 \mathrm{E}-01$ & $9.972 \mathrm{E}+05$ \\
\hline \multirow[t]{4}{*}{$d^{2} P^{e} \rightarrow w^{2} D^{\circ}$} & 0.3936 & 0.2762 & $1.174 \mathrm{E}-01$ & 6 & 10 & $3.439 \mathrm{E}-02$ & $5.272 \mathrm{E}+00$ & $2.285 \mathrm{E}+06$ \\
\hline & 145505.740 & 158715.460 & $1.204 \mathrm{E}-01$ & 4 & 6 & $3.173 \mathrm{E}-02$ & $3.163 \mathrm{E}+00$ & $2.463 \mathrm{E}+06$ \\
\hline & 145505.740 & 158215.590 & $1.159 \mathrm{E}-01$ & 4 & 4 & $3.394 \mathrm{E}-03$ & $3.514 \mathrm{E}-01$ & $3.662 \mathrm{E}+05$ \\
\hline & 145877.660 & 158215.590 & $1.125 \mathrm{E}-01$ & 2 & 4 & $3.295 \mathrm{E}-02$ & $1.757 \mathrm{E}+00$ & $1.675 \mathrm{E}+06$ \\
\hline \multirow{5}{*}{$e^{2} P^{e} \rightarrow w^{2} P^{\circ}$} & 0.3380 & 0.2969 & $4.105 E-02$ & 6 & 6 & $2.535 \mathrm{E}-02$ & $1.112 \mathrm{E}+01$ & $3.432 E+05$ \\
\hline & 151910.830 & 156276.830 & $3.980 \mathrm{E}-02$ & 4 & 4 & $2.048 \mathrm{E}-02$ & $6.175 E+00$ & $2.606 \mathrm{E}+05$ \\
\hline & 151910.830 & 156167.040 & $3.880 \mathrm{E}-02$ & 4 & 2 & $3.993 \mathrm{E}-03$ & $1.235 \mathrm{E}+00$ & $9.658 \mathrm{E}+04$ \\
\hline & 151383.810 & 156276.830 & $4.460 \mathrm{E}-02$ & 2 & 4 & $9.181 E-03$ & $1.235 \mathrm{E}+00$ & $7.334 \mathrm{E}+04$ \\
\hline & 151383.810 & 156167.040 & $4.360 \mathrm{E}-02$ & 2 & 2 & $1.795 \mathrm{E}-02$ & $2.470 \mathrm{E}+00$ & $2.741 E+05$ \\
\hline \multirow[t]{4}{*}{$e^{2} P^{e} \rightarrow w^{2} D^{\circ}$} & 0.3380 & 0.2762 & $6.179 \mathrm{E}-02$ & 6 & 10 & $8.305 \mathrm{E}-01$ & $2.419 E+02$ & $1.528 \mathrm{E}+07$ \\
\hline & 151910.830 & 158715.460 & $6.200 \mathrm{E}-02$ & 4 & 6 & $7.500 \mathrm{E}-01$ & $1.452 \mathrm{E}+02$ & $1.544 \mathrm{E}+07$ \\
\hline & 151910.830 & 158215.590 & $5.750 \mathrm{E}-02$ & 4 & 4 & $7.729 \mathrm{E}-02$ & $1.613 E+01$ & $2.053 E+06$ \\
\hline & 151383.810 & 158215.590 & $6.230 \mathrm{E}-02$ & 2 & 4 & $8.374 E-01$ & $8.065 \mathrm{E}+01$ & $1.305 \mathrm{E}+07$ \\
\hline \multirow[t]{4}{*}{$a^{2} D^{c} \rightarrow y^{2} P^{o}$} & 0.8285 & 0.5055 & $3.230 \mathrm{E}-01$ & 10 & 6 & $5.806 \mathrm{E}-03$ & $5.392 \mathrm{E}-01$ & $8.110 \mathrm{E}+06$ \\
\hline & 97918.860 & 133399.970 & $3.233 E-01$ & 6 & 4 & $5.810 \mathrm{E}-03$ & $3.235 \mathrm{E}-01$ & $7.317 \mathrm{E}+06$ \\
\hline & 97890.740 & 133399.970 & $3.236 \mathrm{E}-01$ & 4 & 4 & $9.691 \mathrm{E}-04$ & $3.594 \mathrm{E}-02$ & $8.149 \mathrm{E}+05$ \\
\hline & 97890.740 & 133268.680 & $3.224 \mathrm{E}-01$ & 4 & 2 & $4.828 \mathrm{E}-03$ & $1.797 \mathrm{E}-01$ & $8.059 \mathrm{E}+06$ \\
\hline \multirow[t]{4}{*}{$a^{2} D^{e} \rightarrow x^{2} P^{0}$} & 0.8285 & 0.4123 & $4.162 \mathrm{E}-01$ & 10 & 6 & $1.561 \mathrm{E}-02$ & $1.125 \mathrm{E}+00$ & $3.619 E+07$ \\
\hline & 97918.860 & 143623.560 & $4.165 \mathrm{E}-01$ & 6 & 4 & $1.562 \mathrm{E}-02$ & $6.751 \mathrm{E}-01$ & $3.265 \mathrm{E}+07$ \\
\hline & 97890.740 & 143623.560 & $4.168 \mathrm{E}-01$ & 4 & 4 & $2.605 E-03$ & $7.501 \mathrm{E}-02$ & $3.634 E+06$ \\
\hline & 97890.740 & 143488.950 & $4.156 \mathrm{E}-01$ & 4 & 2 & $1.299 \mathrm{E}-02$ & $3.750 \mathrm{E}-01$ & $3.603 \mathrm{E}+07$ \\
\hline \multirow[t]{4}{*}{$a^{2} D^{c} \rightarrow w^{2} p^{o}$} & 0.8285 & 0.2969 & $5.316 \mathrm{E}-01$ & 10 & 6 & $3.318 \mathrm{E}-03$ & $1.873 \mathrm{E}-01$ & $1.255 \mathrm{E}+07$ \\
\hline & 97918.860 & 156276.830 & $5.318 \mathrm{E}-01$ & 6 & 4 & $3.320 \mathrm{E}-03$ & $1.124 \mathrm{E}-01$ & $1.131 \mathrm{E}+07$ \\
\hline & 97890.740 & 156276.830 & $5.321 \mathrm{E}-01$ & 4 & 4 & $5.535 \mathrm{E}-04$ & $1.248 E-02$ & $1.259 \mathrm{E}+06$ \\
\hline & 97890.740 & 156167.040 & $5.311 \mathrm{E}-01$ & 4 & 2 & $2.762 \mathrm{E}-03$ & $6.242 \mathrm{E}-02$ & $1.251 \mathrm{E}+07$ \\
\hline \multirow[t]{5}{*}{$a^{2} D^{e} \rightarrow y^{2} D^{\circ}$} & 0.8285 & 0.5272 & $3.013 E-01$ & 10 & 10 & $3.243 E-04$ & $3.229 \mathrm{E}-02$ & $2.364 \mathrm{E}+05$ \\
\hline & 97918.860 & 131187.190 & $3.032 E-01$ & 6 & 6 & $3.046 \mathrm{E}-04$ & $1.808 \mathrm{E}-02$ & $2.249 E+05$ \\
\hline & 97918.860 & 130641.110 & $2.982 \mathrm{E}-01$ & 6 & 4 & $2.140 \mathrm{E}-05$ & $1.292 \mathrm{E}-03$ & $2.293 E+04$ \\
\hline & 97890.740 & 131187.190 & $3.035 \mathrm{E}-01$ & 4 & 6 & $3.266 \mathrm{E}-05$ & $1.292 \mathrm{E}-03$ & $1.610 \mathrm{E}+04$ \\
\hline & 97890.740 & 130641.110 & $2.985 \mathrm{E}-01$ & 4 & 4 & $2.891 \mathrm{E}-04$ & $1.162 \mathrm{E}-02$ & $2.068 \mathrm{E}+05$ \\
\hline \multirow[t]{5}{*}{$a^{2} D^{\circ} \rightarrow x^{2} D^{\circ}$} & 0.8285 & 0.4383 & $3.902 \mathrm{E}-01$ & 10 & 10 & $1.701 \mathrm{E}-02$ & $1.308 \mathrm{E}+00$ & $2.081 \mathrm{E}+07$ \\
\hline & 97918.860 & 140708.890 & $3.899 E-01$ & 6 & 6 & $1.587 \mathrm{E}-02$ & $7.326 \mathrm{E}-01$ & $1.938 \mathrm{E}+07$ \\
\hline & 97918.860 & 140750.340 & $3.903 \mathrm{E}-01$ & 6 & 4 & $1.135 \mathrm{E}-03$ & $5.233 E-02$ & $2.082 E+06$ \\
\hline & 97890.740 & 140708.890 & $3.902 \mathrm{E}-01$ & 4 & 6 & $1.701 \mathrm{E}-03$ & $5.233 \mathrm{E}-02$ & $1.387 \mathrm{E}+06$ \\
\hline & 97890.740 & 140750.340 & $3.906 \mathrm{E}-01$ & 4 & 4 & $1.533 \mathrm{E}-02$ & $4.709 \mathrm{E}-01$ & $1.878 \mathrm{E}+07$ \\
\hline \multirow[t]{5}{*}{$a^{2} D^{c} \rightarrow w^{2} D^{\circ}$} & 0.8285 & 0.2762 & $5.523 \mathrm{E}-01$ & 10 & 10 & $3.976 \mathrm{E}-04$ & $2.159 \mathrm{E}-02$ & $9.741 E+05$ \\
\hline & 97918.860 & 158715.460 & $5.540 \mathrm{E}-01$ & 6 & 6 & $3.722 \mathrm{E}-04$ & $1.209 \mathrm{E}-02$ & $9.175 \mathrm{E}+05$ \\
\hline & 97918.860 & 158215.590 & $5.495 \mathrm{E}-01$ & 6 & 4 & $2.637 \mathrm{E}-05$ & $8.638 \mathrm{E}-04$ & $9.593 \mathrm{E}+04$ \\
\hline & 97890.740 & 158715.460 & $5.543 \mathrm{E}-01$ & 4 & 6 & $3.990 \mathrm{E}-05$ & $8.638 \mathrm{E}-04$ & $6.563 E+04$ \\
\hline & 97890.740 & 158215.590 & $5.498 \mathrm{E}-01$ & 4 & 4 & $3.561 \mathrm{E}-04$ & $7.774 \mathrm{E}-03$ & $8.646 \mathrm{E}+05$ \\
\hline \multirow[t]{4}{*}{$a^{2} D^{e} \rightarrow z^{2} F^{0}$} & 0.8285 & 0.4424 & $3.861 \mathrm{E}-01$ & 10 & 14 & $5.663 \mathrm{E}-03$ & $4.400 \mathrm{E}-01$ & $4.844 \mathrm{E}+06$ \\
\hline & 97918.860 & 140319.230 & $3.864 \mathrm{E}-01$ & 6 & 8 & $5.397 \mathrm{E}-03$ & $2.514 \mathrm{E}-01$ & $4.854 \mathrm{E}+06$ \\
\hline & 97918.860 & 140230.100 & $3.856 \mathrm{E}-01$ & 6 & 6 & $2.693 \mathrm{E}-04$ & $1.257 \mathrm{E}-02$ & $3.216 \mathrm{E}+05$ \\
\hline & 97890.740 & 140230.100 & $3.859 \mathrm{E}-01$ & 4 & 6 & $5.659 \mathrm{E}-03$ & $1.760 \mathrm{E}-01$ & $4.512 \mathrm{E}+06$ \\
\hline
\end{tabular}


Table IV. Continued

\begin{tabular}{|c|c|c|c|c|c|c|c|c|}
\hline Transition & $\begin{array}{l}E_{i} \\
\mathrm{~cm}^{-1}\end{array}$ & $\begin{array}{l}E_{f} \\
\mathrm{~cm}^{-1}\end{array}$ & $\begin{array}{l}E_{f i} \\
\mathrm{Ry}\end{array}$ & $g_{i}$ & $g_{f}$ & $f_{i f}$ & $S$ & $\begin{array}{l}A_{f i} \\
\mathbf{s}^{-1}\end{array}$ \\
\hline \multirow[t]{4}{*}{$a^{2} D^{\circ} \rightarrow y^{2} F^{o}$} & 0.8285 & 0.1856 & $6.429 \mathrm{E}-01$ & 10 & 14 & $3.644 \mathrm{E}-04$ & $1.700 \mathrm{E}-02$ & $8.641 \mathrm{E}+05$ \\
\hline & 97918.860 & 168473.480 & $6.429 \mathrm{E}-01$ & 6 & 8 & $3.470 \mathrm{E}-04$ & $9.716 \mathrm{E}-03$ & $8.640 \mathrm{E}+05$ \\
\hline & 97918.860 & 168443.140 & $6.427 \mathrm{E}-01$ & 6 & 6 & $1.735 \mathrm{E}-05$ & $4.858 \mathrm{E}-04$ & $5.755 \mathrm{E}+04$ \\
\hline & 97890.740 & 168443.140 & $6.429 \mathrm{E}-01$ & 4 & 6 & $3.644 \mathrm{E}-04$ & $6.801 E-03$ & $8.066 \mathrm{E}+05$ \\
\hline \multirow[t]{4}{*}{$b^{2} D^{\circ} \rightarrow y^{2} P^{\circ}$} & 0.6132 & 0.5055 & $1.078 \mathrm{E}-01$ & 10 & 6 & $1.586 \mathrm{E}-02$ & $4.414 \mathrm{E}+\infty$ & $2.466 \mathrm{E}+06$ \\
\hline & 121530.020 & 133399.970 & $1.081 \mathrm{E}-01$ & 6 & 4 & $1.591 \mathrm{E}-02$ & $2.648 E+00$ & $2.239 \mathrm{E}+06$ \\
\hline & 121528.720 & 133399.970 & $1.081 \mathrm{E}-01$ & 4 & 4 & $2.651 \mathrm{E}-03$ & $2.943 \mathrm{E}-01$ & $2.488 \mathrm{E}+05$ \\
\hline & 121528.720 & 133268.680 & $1.069 \mathrm{E}-01$ & 4 & 2 & $1.311 \mathrm{E}-02$ & $1.471 \mathrm{E}+\infty 0$ & $2.406 \mathrm{E}+06$ \\
\hline \multirow[t]{4}{*}{$\mathrm{b}^{2} \mathrm{D}^{\mathrm{e}} \rightarrow \mathrm{x}^{2} \mathrm{P}^{\circ}$} & 0.6132 & 0.4123 & $2.009 \mathrm{E}-01$ & 10 & 6 & $1.706 \mathrm{E}-01$ & $2.547 \mathrm{E}+01$ & $9.220 \mathrm{E}+07$ \\
\hline & 121530.020 & 143623.560 & $2.013 \mathrm{E}-01$ & 6 & 4 & $1.709 \mathrm{E}-01$ & $1.528 \mathrm{E}+01$ & $8.344 \mathrm{E}+07$ \\
\hline & 121528.720 & 143623.560 & $2.013 \mathrm{E}-01$ & 4 & 4 & $2.849 \mathrm{E}-02$ & $1.698 \mathrm{E}+00$ & $9.272 \mathrm{E}+06$ \\
\hline & 121528.720 & 143488.950 & $2.001 \mathrm{E}-01$ & 4 & 2 & $1.416 \mathrm{E}-01$ & $8.491 \mathrm{E}+00$ & $9.107 \mathrm{E}+07$ \\
\hline \multirow[t]{4}{*}{$\mathrm{b}^{2} \mathrm{D}^{\mathrm{e}} \rightarrow \mathrm{w}^{2} \mathrm{P}^{\mathrm{o}}$} & 0.6132 & 0.2969 & $3.163 \mathrm{E}-01$ & 10 & 6 & $4.107 \mathrm{E}-03$ & $3.895 \mathrm{E}-01$ & $5.501 \mathrm{E}+06$ \\
\hline & 121530.020 & 156276.830 & $3.166 \mathrm{E}-01$ & 6 & 4 & $4.111 \mathrm{E}-03$ & $2.337 \mathrm{E}-01$ & $4.964 \mathrm{E}+06$ \\
\hline & 121528.720 & 156276.830 & $3.166 \mathrm{E}-01$ & 4 & 4 & $6.851 \mathrm{E}-04$ & $2.597 \mathrm{E}-02$ & $5.516 \mathrm{E}+05$ \\
\hline & 121528.720 & 156167.040 & $3.156 E-01$ & 4 & 2 & $3.415 \mathrm{E}-03$ & $1.298 \mathrm{E}-01$ & $5.464 \mathrm{E}+06$ \\
\hline \multirow[t]{5}{*}{$b^{2} D^{e} \rightarrow y^{2} D^{a}$} & 0.6132 & 0.5272 & $8.602 \mathrm{E}-02$ & 10 & 10 & $2.472 \mathrm{E}-02$ & $8.621 E+00$ & $1.469 \mathrm{E}+06$ \\
\hline & 121530.020 & 131187.190 & $8.800 \mathrm{E}-02$ & 6 & 6 & $2.360 \mathrm{E}-02$ & $4.828 \mathrm{E}+\infty 0$ & $1.468 \mathrm{E}+06$ \\
\hline & 121530.020 & 130641.110 & $8.300 E-02$ & 6 & 4 & $1.590 \mathrm{E}-03$ & $3.449 E-01$ & $1.320 \mathrm{E}+05$ \\
\hline & 121528.720 & 131187.190 & $8.800 \mathrm{E}-02$ & 4 & 6 & $2.529 \mathrm{E}-03$ & $3.449 \mathrm{E}-01$ & $1.049 E+05$ \\
\hline & 121528.720 & 130641.110 & $8.300 \mathrm{E}-02$ & 4 & 4 & $2.147 \mathrm{E}-02$ & $3.104 E+00$ & $1.188 \mathrm{E}+06$ \\
\hline \multirow[t]{5}{*}{$b^{2} D^{\circ} \rightarrow x^{2} D^{\circ}$} & 0.6132 & 0.4383 & $1.749 \mathrm{E}-01$ & 10 & 10 & $3.508 \mathrm{E}-01$ & $6.016 \mathrm{E}+01$ & $8.622 \mathrm{E}+07$ \\
\hline & 121530.020 & 140708.890 & $1.747 \mathrm{E}-01$ & 6 & 6 & $3.270 \mathrm{E}-01$ & $3.369 \mathrm{E}+01$ & $8.016 \mathrm{E}+07$ \\
\hline & 121530.020 & 140750.340 & $1.751 \mathrm{E}-01$ & 6 & 4 & $2.341 \mathrm{E}-02$ & $2.406 \mathrm{E}+00$ & $8.647 \mathrm{E}+06$ \\
\hline & 121528.720 & 140708.890 & $1.747 \mathrm{E}-01$ & 4 & 6 & $3.503 E-02$ & $2.406 \mathrm{E}+00$ & $5.725 \mathrm{E}+06$ \\
\hline & 121528.720 & 140750.340 & $1.751 \mathrm{E}-01$ & 4 & 4 & $3.160 E-01$ & $2.166 \mathrm{E}+01$ & $7.783 \mathrm{E}+07$ \\
\hline \multirow[t]{5}{*}{$b^{2} D^{e} \rightarrow w^{2} D^{\circ}$} & 0.6132 & 0.2762 & $3.370 \mathrm{E}-01$ & 10 & 10 & $8.355 E-03$ & $7.437 \mathrm{E}-01$ & $7.623 \mathrm{E}+06$ \\
\hline & 121530.020 & 158715.460 & $3.388 \mathrm{E}-01$ & 6 & 6 & $7.838 \mathrm{E}-03$ & $4.164 \mathrm{E}-01$ & $7.227 \mathrm{E}+06$ \\
\hline & 121530.020 & 158215.590 & $3.343 \mathrm{E}-01$ & 6 & 4 & $5.525 \mathrm{E}-04$ & $2.975 \mathrm{E}-02$ & $7.439 E+05$ \\
\hline & 121528.720 & 158715.460 & $3.388 \mathrm{E}-01$ & 4 & 6 & $8.398 \mathrm{E}-04$ & $2.975 \mathrm{E}-02$ & $5.162 \mathrm{E}+05$ \\
\hline & 121528.720 & 158215.590 & $3.343 \mathrm{E}-01$ & 4 & 4 & $7.458 \mathrm{E}-03$ & $2.677 \mathrm{E}-01$ & $6.695 \mathrm{E}+06$ \\
\hline \multirow[t]{4}{*}{$b^{2} D^{e} \rightarrow z^{2} F^{o}$} & 0.6132 & 0.4424 & $1.709 \mathrm{E}-01$ & 10 & 14 & $4.751 \mathrm{E}-01$ & $8.341 E+01$ & $7.959 \mathrm{E}+07$ \\
\hline & 121530.020 & 140319.230 & $1.712 \mathrm{E}-01$ & 6 & 8 & $4.533 \mathrm{E}-01$ & $4.766 \mathrm{E}+01$ & $8.004 \mathrm{E}+07$ \\
\hline & 121530.020 & 140230.100 & $1.704 \mathrm{E}-01$ & 6 & 6 & $2.256 \mathrm{E}-02$ & $2.383 \mathrm{E}+00$ & $5.261 E+06$ \\
\hline & 121528.720 & 140230.100 & $1.704 \mathrm{E}-01$ & 4 & 6 & $4.737 \mathrm{E}-01$ & $3.336 \mathrm{E}+01$ & $7.366 \mathrm{E}+07$ \\
\hline \multirow[t]{4}{*}{$\mathrm{b}^{2} \mathrm{D}^{\mathrm{s}} \rightarrow \mathrm{y}^{2} \mathrm{~F}^{\circ}$} & 0.6132 & 0.1856 & $4.277 \mathrm{E}-01$ & 10 & 14 & $1.388 \mathrm{E}-03$ & $9.733 \mathrm{E}-02$ & $1.456 \mathrm{E}+06$ \\
\hline & 121530.020 & 168473.480 & $4.277 \mathrm{E}-01$ & 6 & 8 & $1.322 \mathrm{E}-03$ & $5.562 \mathrm{E}-02$ & $1.456 \mathrm{E}+06$ \\
\hline & 121530.020 & 168443.140 & $4.275 \mathrm{E}-01$ & 6 & 6 & $6.605 \mathrm{E}-05$ & $2.781 \mathrm{E}-03$ & $9.695 \mathrm{E}+04$ \\
\hline & 121528.720 & 168443.140 & $4.275 \mathrm{E}-01$ & 4 & 6 & $1.387 \mathrm{E}-03$ & $3.893 \mathrm{E}-02$ & $1.357 \mathrm{E}+06$ \\
\hline \multirow[t]{4}{*}{$c^{2} D^{\circ} \rightarrow x^{2} P^{\circ}$} & 0.5029 & 0.4123 & $9.063 \mathrm{E}-02$ & 10 & 6 & $2.318 \mathrm{E}-02$ & $7.673 E+00$ & $2.549 \mathrm{E}+06$ \\
\hline & 133814.840 & 143623.560 & $8.940 \mathrm{E}-02$ & 6 & 4 & $2.287 \mathrm{E}-02$ & $4.604 \mathrm{E}+00$ & $2.202 \mathrm{E}+06$ \\
\hline & 133360.860 & 143623.560 & $9.350 \mathrm{E}-02$ & 4 & 4 & $3.986 \mathrm{E}-03$ & $5.115 \mathrm{E}-01$ & $2.799 E+05$ \\
\hline & 133360.860 & 143488.950 & $9.230 \mathrm{E}-02$ & 4 & 2 & $1.967 \mathrm{E}-02$ & $2.558 \mathrm{E}+00$ & $2.692 \mathrm{E}+06$ \\
\hline \multirow[t]{4}{*}{$c^{2} D^{e} \rightarrow w^{2} P^{o}$} & 0.5029 & 0.2969 & $2.060 \mathrm{E}-01$ & 10 & 6 & $5.409 E-03$ & $7.877 \mathrm{E}-01$ & $3.073 \mathrm{E}+06$ \\
\hline & 133814.840 & 156276.830 & $2.047 \mathrm{E}-01$ & 6 & 4 & $5.375 \mathrm{E}-03$ & $4.726 \mathrm{E}-01$ & $2.713 \mathrm{E}+06$ \\
\hline & 133360.860 & 156276.830 & $2.088 \mathrm{E}-01$ & 4 & 4 & $9.137 \mathrm{E}-04$ & $5.251 \mathrm{E}-02$ & $3.200 \mathrm{E}+05$ \\
\hline & 133360.860 & 156167.040 & $2.078 \mathrm{E}-01$ & 4 & 2 & $4.547 E-03$ & $2.626 \mathrm{E}-01$ & $3.154 \mathrm{E}+06$ \\
\hline \multirow[t]{5}{*}{$c^{2} D^{e} \rightarrow x^{2} D^{\circ}$} & 0.5029 & 0.4383 & $6.463 \mathrm{E}-02$ & 10 & 10 & $5.104 \mathrm{E}-03$ & $2.369 \mathrm{E}+00$ & $1.713 E+05$ \\
\hline & 133814.840 & 140708.890 & $6.280 \mathrm{E}-02$ & 6 & 6 & $4.629 E-03$ & $1.327 \mathrm{E}+\infty 0$ & $1.466 \mathrm{E}+05$ \\
\hline & 133814.840 & 140750.340 & $6.320 \mathrm{E}-02$ & 6 & 4 & $3.328 \mathrm{E}-04$ & $9.477 \mathrm{E}-02$ & $1.601 E+04$ \\
\hline & 133360.860 & 140708.890 & $6.690 \mathrm{E}-02$ & 4 & 6 & $5.284 \mathrm{E}-04$ & $9.477 \mathrm{E}-02$ & $1.266 \mathrm{E}+04$ \\
\hline & 133360.860 & 140750.340 & $6.730 \mathrm{E}-02$ & 4 & 4 & $4.784 \mathrm{E}-03$ & $8.530 \mathrm{E}-01$ & $1.740 \mathrm{E}+05$ \\
\hline
\end{tabular}


Table IV. Continued

\begin{tabular}{|c|c|c|c|c|c|c|c|c|}
\hline Transition & $\begin{array}{l}E_{i} \\
\mathrm{~cm}^{-1}\end{array}$ & $\begin{array}{l}E_{f} \\
\mathrm{~cm}^{-1}\end{array}$ & $\begin{array}{l}E_{f i} \\
\text { Ry }\end{array}$ & $g_{i}$ & $g_{f}$ & $f_{i f}$ & $S$ & $\begin{array}{l}A_{f i} \\
\mathbf{s}^{-1} \\
\end{array}$ \\
\hline \multirow{5}{*}{$c^{2} D^{e} \rightarrow w^{2} D^{\circ}$} & 0.5029 & 0.2762 & $2.267 \mathrm{E}-01$ & 10 & 10 & $5.689 \mathrm{E}-03$ & $7.527 \mathrm{E}-01$ & $2.349 \mathrm{E}+06$ \\
\hline & 133814.840 & 158715.460 & $2.269 \mathrm{E}-01$ & 6 & 6 & $5.313 \mathrm{E}-03$ & $4.215 \mathrm{E}-01$ & $2.197 \mathrm{E}+06$ \\
\hline & 133814.840 & 158215.590 & $2.224 \mathrm{E}-01$ & 6 & 4 & $3.720 \mathrm{E}-04$ & $3.011 \mathrm{E}-02$ & $2.217 \mathrm{E}+05$ \\
\hline & 133360.860 & 158715.460 & $2.310 \mathrm{E}-01$ & 4 & 6 & $5.796 \mathrm{E}-04$ & $3.011 \mathrm{E}-02$ & $1.656 \mathrm{E}+05$ \\
\hline & 133360.860 & 158215.590 & $2.265 \mathrm{E}-01$ & 4 & 4 & $5.115 \mathrm{E}-03$ & $2.710 \mathrm{E}-01$ & $2.108 \mathrm{E}+06$ \\
\hline \multirow[t]{4}{*}{$c^{2} D^{e} \rightarrow z^{2} F^{\circ}$} & 0.5029 & 0.4424 & $6.058 \mathrm{E}-02$ & 10 & 14 & $1.762 \mathrm{E}-03$ & $8.728 \mathrm{E}-01$ & $3.711 \mathrm{E}+04$ \\
\hline & 133814.840 & 140319.230 & $5.930 \mathrm{E}-02$ & 6 & 8 & $1.643 \mathrm{E}-03$ & $4.988 \mathrm{E}-01$ & $3.481 \mathrm{E}+04$ \\
\hline & 133814.840 & 140230.100 & $5.850 \mathrm{E}-02$ & 6 & 6 & $8.105 E-05$ & $2.494 \mathrm{E}-02$ & $2.228 E+03$ \\
\hline & 133360.860 & 140230.100 & $6.260 \mathrm{E}-02$ & 4 & 6 & $1.821 \mathrm{E}-03$ & $3.491 \mathrm{E}-01$ & $3.822 \mathrm{E}+04$ \\
\hline \multirow[t]{4}{*}{$c^{2} D^{\circ} \rightarrow y^{2} F^{\circ}$} & 0.5029 & 0.1856 & $3.174 \mathrm{E}-01$ & 10 & 14 & $1.032 \mathrm{E}-03$ & $9.755 \mathrm{E}-02$ & $5.963 \mathrm{E}+05$ \\
\hline & 133814.840 & 168473.480 & $3.158 \mathrm{E}-01$ & 6 & 8 & $9.779 \mathrm{E}-04$ & $5.574 \mathrm{E}-02$ & $5.875 \mathrm{E}+05$ \\
\hline & 133814.840 & 168443.140 & $3.156 \mathrm{E}-01$ & 6 & 6 & $4.887 \mathrm{E}-05$ & $2.787 \mathrm{E}-03$ & $3.909 \mathrm{E}+04$ \\
\hline & 133360.860 & 168443.140 & $3.197 \mathrm{E}-01$ & 4 & 6 & $1.040 \mathrm{E}-03$ & $3.902 \mathrm{E}-02$ & $5.689 \mathrm{E}+05$ \\
\hline \multirow[t]{4}{*}{$d^{2} D^{e} \rightarrow w^{2} P^{o}$} & 0.4079 & 0.2969 & $1.110 \mathrm{E}-01$ & 10 & 6 & $3.171 \mathrm{E}-02$ & $8.571 \mathrm{E}+\infty$ & $5.227 \mathrm{E}+06$ \\
\hline & 144009.420 & 156276.830 & $1.118 \mathrm{E}-01$ & 6 & 4 & $3.194 \mathrm{E}-02$ & $5.143 E+\infty$ & $4.810 \mathrm{E}+06$ \\
\hline & 144142.160 & 156276.830 & $1.106 \mathrm{E}-01$ & 4 & 4 & $5.267 \mathrm{E}-03$ & $5.714 \mathrm{E}-01$ & $5.175 \mathrm{E}+05$ \\
\hline & 144142.160 & 156167.040 & $1.096 \mathrm{E}-01$ & 4 & 2 & $2.610 \mathrm{E}-02$ & $2.857 \mathrm{E}+\infty 0$ & $5.036 \mathrm{E}+06$ \\
\hline \multirow{5}{*}{$d^{2} D^{\circ} \rightarrow w^{2} D^{0}$} & 0.4079 & 0.2762 & $1.317 \mathrm{E}-01$ & 10 & 10 & $4.828 \mathrm{E}-04$ & $1.100 \mathrm{E}-01$ & $6.727 \mathrm{E}+04$ \\
\hline & 144009.420 & 158715.460 & $1.340 \mathrm{E}-01$ & 6 & 6 & $4.585 \mathrm{E}-04$ & $6.159 E-02$ & $6.613 E+04$ \\
\hline & 144009.420 & 158215.590 & $1.295 \mathrm{E}-01$ & 6 & 4 & $3.165 \mathrm{E}-05$ & $4.399 \mathrm{E}-03$ & $6.395 \mathrm{E}+03$ \\
\hline & 144142.160 & 158715.460 & $1.328 \mathrm{E}-01$ & 4 & 6 & $4.869 \mathrm{E}-05$ & $4.399 \mathrm{E}-03$ & $4.598 \mathrm{E}+03$ \\
\hline & 144142.160 & 158215.590 & $1.283 \mathrm{E}-01$ & 4 & 4 & $4.233 \mathrm{E}-04$ & $3.959 \mathrm{E}-02$ & $5.597 \mathrm{E}+04$ \\
\hline \multirow[t]{4}{*}{$\mathrm{d}^{2} \mathrm{D}^{\mathrm{e}} \rightarrow \mathrm{y}^{2} \mathrm{~F}^{\mathrm{o}}$} & 0.4079 & 0.1856 & $2.223 \mathrm{E}-01$ & 10 & 14 & $1.399 \mathrm{E}-02$ & $1.887 \mathrm{E}+\infty$ & $3.966 \mathrm{E}+06$ \\
\hline & 144009.420 & 168473.480 & $2.229 \mathrm{E}-01$ & 6 & 8 & $1.335 \mathrm{E}-02$ & $1.078 \mathrm{E}+00$ & $3.997 \mathrm{E}+06$ \\
\hline & 144009.420 & 168443.140 & $2.227 \mathrm{E}-01$ & 6 & 6 & $6.671 E-04$ & $5.392 \mathrm{E}-02$ & $2.657 \mathrm{E}+05$ \\
\hline & 144142.160 & 168443.140 & $2.215 E-01$ & 4 & 6 & $1.393 \mathrm{E}-02$ & $7.548 \mathrm{E}-01$ & $3.660 \mathrm{E}+06$ \\
\hline \multirow[t]{4}{*}{$\mathrm{e}^{2} \mathrm{D}^{\mathrm{e}} \rightarrow \mathrm{w}^{2} \mathrm{P}^{\circ}$} & 0.3639 & 0.2969 & $6.696 \mathrm{E}-02$ & 10 & 6 & $7.154 \mathrm{E}-02$ & $3.205 \mathrm{E}+01$ & $4.294 \mathrm{E}+06$ \\
\hline & 148886.570 & 156276.830 & $6.730 \mathrm{E}-02$ & 6 & 4 & $7.190 \mathrm{E}-02$ & $1.923 \mathrm{E}+01$ & $3.924 \mathrm{E}+06$ \\
\hline & 148900.910 & 156276.830 & $6.720 \mathrm{E}-02$ & 4 & 4 & $1.197 \mathrm{E}-02$ & $2.137 \mathrm{E}+00$ & $4.340 \mathrm{E}+05$ \\
\hline & 148900.910 & 156167.040 & $6.620 E-02$ & 4 & 2 & $5.894 \mathrm{E}-02$ & $1.068 \mathrm{E}+01$ & $4.149 E+06$ \\
\hline \multirow[t]{5}{*}{$\mathrm{e}^{2} \mathrm{D}^{\mathrm{e}} \rightarrow \mathrm{w}^{2} \mathrm{D}^{\circ}$} & 0.3639 & 0.2762 & $8.769 \mathrm{E}-02$ & 10 & 10 & $4.617 \mathrm{E}-02$ & $1.580 \mathrm{E}+01$ & $2.852 E+06$ \\
\hline & 148886.570 & 158715.460 & $8.950 \mathrm{E}-02$ & 6 & 6 & $4.398 \mathrm{E}-02$ & $8.845 \mathrm{E}+00$ & $2.830 \mathrm{E}+06$ \\
\hline & 148886.570 & 158215.590 & $8.500 \mathrm{E}-02$ & 6 & 4 & $2.984 \mathrm{E}-03$ & $6.318 \mathrm{E}-01$ & $2.597 \mathrm{E}+05$ \\
\hline & 148900.910 & 158715.460 & $8.940 E-02$ & 4 & 6 & $4.707 \mathrm{E}-03$ & $6.318 \mathrm{E}-01$ & $2.014 \mathrm{E}+05$ \\
\hline & 148900.910 & 158215.590 & $8.490 \mathrm{E}-02$ & 4 & 4 & $4.023 \mathrm{E}-02$ & $5.686 \mathrm{E}+00$ & $2.329 \mathrm{E}+06$ \\
\hline \multirow[t]{4}{*}{$e^{2} D^{e} \rightarrow y^{2} F^{0}$} & 0.3639 & 0.1856 & $1.783 E-01$ & 10 & 14 & $4.233 \mathrm{E}-03$ & $7.121 \mathrm{E}-01$ & $7.721 \mathrm{E}+05$ \\
\hline & 148886.570 & 168473.480 & $1.784 \mathrm{E}-01$ & 6 & 8 & $4.033 \mathrm{E}-03$ & $4.069 \mathrm{E}-01$ & $7.732 \mathrm{E}+05$ \\
\hline & 148886.570 & 168443.140 & $1.782 \mathrm{E}-01$ & 6 & 6 & $2.014 \mathrm{E}-04$ & $2.034 \mathrm{E}-02$ & $5.137 \mathrm{E}+04$ \\
\hline & 148900.910 & 168443.140 & $1.781 \mathrm{E}-01$ & 4 & 6 & $4.227 \mathrm{E}-03$ & $2.848 \mathrm{E}-01$ & $7.180 \mathrm{E}+05$ \\
\hline \multirow[t]{4}{*}{$\mathrm{f}^{2} \mathrm{D}^{\mathrm{e}} \rightarrow \mathrm{y}^{2} \mathrm{~F}^{\circ}$} & 0.2673 & 0.1856 & $8.171 E-02$ & 10 & 14 & $8.192 \mathrm{E}-03$ & $3.008 \mathrm{E}+00$ & $3.138 E+05$ \\
\hline & 159492.830 & 168473.480 & $8.180 \mathrm{E}-02$ & 6 & 8 & $7.810 \mathrm{E}-03$ & $1.719 \mathrm{E}+00$ & $3.148 \mathrm{E}+05$ \\
\hline & 159492.830 & 168443.140 & $8.160 \mathrm{E}-02$ & 6 & 6 & $3.896 \mathrm{E}-04$ & $8.593 \mathrm{E}-02$ & $2.084 E+04$ \\
\hline & 159495.940 & 168443.140 & $8.160 \mathrm{E}-02$ & 4 & 6 & $8.181 \mathrm{E}-03$ & $1.203 \mathrm{E}+00$ & $2.917 \mathrm{E}+05$ \\
\hline \multirow[t]{4}{*}{$\mathrm{g}^{2} \mathrm{D}^{\mathrm{e}} \rightarrow \mathrm{y}^{2} \mathrm{~F}^{\circ}$} & 0.2469 & 0.1856 & $6.129 \mathrm{E}-02$ & 10 & 14 & $7.049 \mathrm{E}-01$ & $3.450 \mathrm{E}+02$ & $1.519 E+07$ \\
\hline & 161733.100 & 168473.480 & $6.140 \mathrm{E}-02$ & 6 & 8 & $6.726 \mathrm{E}-01$ & $1.972 \mathrm{E}+02$ & $1.527 \mathrm{E}+07$ \\
\hline & 161733.100 & 168443.140 & $6.120 \mathrm{E}-02$ & 6 & 6 & $3.352 \mathrm{E}-02$ & $9.859 \mathrm{E}+00$ & $1.008 \mathrm{E}+06$ \\
\hline & 161737.990 & 168443.140 & $6.110 \mathrm{E}-02$ & 4 & 6 & $7.027 \mathrm{E}-01$ & $1.380 \mathrm{E}+02$ & $1.405 E+07$ \\
\hline
\end{tabular}


Table IV. Continued

\begin{tabular}{|c|c|c|c|c|c|c|c|c|}
\hline Transition & $\begin{array}{l}E_{i} \\
\mathrm{~cm}^{-1} \\
\end{array}$ & $\begin{array}{l}E_{f} \\
\mathrm{~cm}^{-1}\end{array}$ & $\begin{array}{l}E_{f i} \\
\text { Ry }\end{array}$ & $g_{i}$ & $g_{f}$ & $f_{i f}$ & $S$ & $\begin{array}{l}A_{f i} \\
\mathrm{~s}^{-1} \\
\end{array}$ \\
\hline \multirow[t]{4}{*}{$\mathrm{h}^{2} \mathrm{D}^{\mathrm{e}} \rightarrow \mathrm{y}^{2} \mathrm{~F}^{0}$} & 0.1944 & 0.1856 & $8.818 \mathrm{E}-03$ & 10 & 14 & $1.286 \mathrm{E}-02$ & $4.376 \mathrm{E}+01$ & $5.739 \mathrm{E}+03$ \\
\hline & 167506.390 & 168473.480 & $8.800 \mathrm{E}-03$ & 6 & 8 & $1.223 \mathrm{E}-02$ & $2.501 \mathrm{E}+01$ & $5.704 \mathrm{E}+03$ \\
\hline & 167506.390 & 168443.140 & $8.600 \mathrm{E}-03$ & 6 & 6 & $5.974 \mathrm{E}-04$ & $1.250 \mathrm{E}+\infty 0$ & $3.549 E+02$ \\
\hline & 167472.420 & 168443.140 & $8.900 \mathrm{E}-03$ & 4 & 6 & $1.298 \mathrm{E}-02$ & $1.751 E+01$ & $5.507 \mathrm{E}+03$ \\
\hline \multirow[t]{4}{*}{$\mathrm{a}^{2} \mathrm{~F}^{e} \rightarrow \mathrm{y}^{2} \mathrm{D}^{\circ}$} & 0.6720 & 0.5272 & $1.448 \mathrm{E}-01$ & 14 & 10 & $8.468 \mathrm{E}-02$ & $2.456 \mathrm{E}+01$ & $1.996 \mathrm{E}+07$ \\
\hline & 115285.610 & 131187.190 & $1.449 \mathrm{E}-01$ & 8 & 6 & $8.474 \mathrm{E}-02$ & $1.404 \mathrm{E}+01$ & $1.905 \mathrm{E}+07$ \\
\hline & 114804.370 & 131187.190 & $1.493 \mathrm{E}-01$ & 6 & 6 & $5.821 \mathrm{E}-03$ & $7.018 \mathrm{E}-01$ & $1.042 \mathrm{E}+06$ \\
\hline & 114804.370 & 130641.110 & $1.443 E-01$ & 6 & 4 & $7.876 \mathrm{E}-02$ & $9.825 \mathrm{E}+\infty 0$ & $1.976 \mathrm{E}+07$ \\
\hline \multirow{4}{*}{$a^{2} F^{e} \rightarrow x^{2} D^{\circ}$} & 0.6720 & 0.4383 & $2.337 \mathrm{E}-01$ & 14 & 10 & $1.311 \mathrm{E}-02$ & $2.355 \mathrm{E}+\infty 0$ & $8.049 \mathrm{E}+06$ \\
\hline & 115285.610 & 140708.890 & $2.316 \mathrm{E}-01$ & 8 & 6 & $1.299 \mathrm{E}-02$ & $1.346 \mathrm{E}+00$ & $7.461 E+06$ \\
\hline & 114804.370 & 140708.890 & $2.360 \mathrm{E}-01$ & 6 & 6 & $8.823 E-04$ & $6.729 \mathrm{E}-02$ & $3.947 \mathrm{E}+05$ \\
\hline & 114804.370 & 140750.340 & $2.364 \mathrm{E}-01$ & 6 & 4 & $1.237 \mathrm{E}-02$ & $9.421 \mathrm{E}-01$ & $8.331 \mathrm{E}+06$ \\
\hline \multirow[t]{4}{*}{$\mathrm{a}^{2} \mathrm{~F}^{e} \rightarrow \mathrm{w}^{2} \mathrm{D}^{\circ}$} & 0.6720 & 0.2762 & $3.958 \mathrm{E}-01$ & 14 & 10 & $2.423 \mathrm{E}-03$ & $2.571 \mathrm{E}-01$ & $4.270 \mathrm{E}+06$ \\
\hline & 115285.610 & 158715.460 & $3.957 \mathrm{E}-01$ & 8 & 6 & $2.423 E-03$ & $1.469 \mathrm{E}-01$ & $4.063 \mathrm{E}+06$ \\
\hline & 114804.370 & 158715.460 & $4.001 \mathrm{E}-01$ & 6 & 6 & $1.633 E-04$ & $7.347 \mathrm{E}-03$ & $2.100 \mathrm{E}+05$ \\
\hline & 114804.370 & 158215.590 & $3.956 \mathrm{E}-01$ & 6 & 4 & $2.261 \mathrm{E}-03$ & $1.029 \mathrm{E}-01$ & $4.262 \mathrm{E}+06$ \\
\hline \multirow[t]{5}{*}{$a^{2} F^{e} \rightarrow z^{2} F^{0}$} & 0.6720 & 0.4424 & $2.297 \mathrm{E}-01$ & 14 & 14 & $2.804 \mathrm{E}-02$ & $5.127 \mathrm{E}+\infty 0$ & $1.188 \mathrm{E}+07$ \\
\hline & 115285.610 & 140319.230 & $2.281 \mathrm{E}-01$ & 8 & 8 & $2.685 \mathrm{E}-02$ & $2.825 \mathrm{E}+00$ & $1.122 \mathrm{E}+07$ \\
\hline & 115285.610 & 140230.100 & $2.273 \mathrm{E}-01$ & 8 & 6 & $9.910 \mathrm{E}-04$ & $1.046 \mathrm{E}-01$ & $5.483 \mathrm{E}+05$ \\
\hline & 114804.370 & 140319.230 & $2.325 \mathrm{E}-01$ & 6 & 8 & $1.352 \mathrm{E}-03$ & $1.046 \mathrm{E}-01$ & $4.401 \mathrm{E}+05$ \\
\hline & 114804.370 & 140230.100 & $2.317 \mathrm{E}-01$ & 6 & 6 & $2.721 \mathrm{E}-02$ & $2.114 \mathrm{E}+00$ & $1.173 \mathrm{E}+07$ \\
\hline \multirow[t]{5}{*}{$\mathrm{a}^{2} \mathrm{~F}^{\mathrm{e}} \rightarrow \mathrm{y}^{2} \mathrm{~F}^{\mathrm{o}}$} & 0.6720 & 0.1856 & $4.864 \mathrm{E}-01$ & 14 & 14 & $4.767 \mathrm{E}-03$ & $4.116 \mathrm{E}-01$ & $9.060 \mathrm{E}+06$ \\
\hline & 115285.610 & 168473.480 & $4.846 \mathrm{E}-01$ & 8 & 8 & $4.579 \mathrm{E}-03$ & $2.268 \mathrm{E}-01$ & $8.638 E+06$ \\
\hline & 115285.610 & 168443.140 & $4.844 \mathrm{E}-01$ & 8 & 6 & $1.695 \mathrm{E}-04$ & $8.400 \mathrm{E}-03$ & $4.260 \mathrm{E}+05$ \\
\hline & 114804.370 & 168473.480 & $4.890 \mathrm{E}-01$ & 6 & 8 & $2.282 \mathrm{E}-04$ & $8.400 \mathrm{E}-03$ & $3.287 \mathrm{E}+05$ \\
\hline & 114804.370 & 168443.140 & $4.888 \mathrm{E}-01$ & 6 & 6 & $4.608 \mathrm{E}-03$ & $1.697 \mathrm{E}-01$ & $8.842 E+06$ \\
\hline \multirow[t]{4}{*}{$b^{2} F^{e} \rightarrow x^{2} D^{0}$} & 0.4584 & 0.4383 & $2.010 \mathrm{E}-02$ & 14 & 10 & $2.488 \mathrm{E}-02$ & $5.199 \mathrm{E}+01$ & $1.130 \mathrm{E}+05$ \\
\hline & 138527.980 & 140708.890 & $1.980 \mathrm{E}-02$ & 8 & 6 & $2.451 \mathrm{E}-02$ & $2.971 \mathrm{E}+01$ & $1.029 \mathrm{E}+05$ \\
\hline & 138509.170 & 140708.890 & $2.000 \mathrm{E}-02$ & 6 & 6 & $1.650 \mathrm{E}-03$ & $1.485 \mathrm{E}+00$ & $5.303 E+03$ \\
\hline & 138509.170 & 140750.340 & $2.040 \mathrm{E}-02$ & 6 & 4 & $2.357 \mathrm{E}-02$ & $2.080 \mathrm{E}+01$ & $1.182 \mathrm{E}+05$ \\
\hline \multirow[t]{4}{*}{$\mathrm{b}^{2} \mathrm{~F}^{\circ} \rightarrow \mathrm{w}^{2} \mathrm{D}^{\circ}$} & 0.4584 & 0.2762 & $1.822 \mathrm{E}-01$ & 14 & 10 & $2.353 \mathrm{E}-02$ & $5.423 \mathrm{E}+00$ & $8.784 \mathrm{E}+06$ \\
\hline & 138527.980 & 158715.460 & $1.839 \mathrm{E}-01$ & 8 & 6 & $2.374 \mathrm{E}-02$ & $3.099 \mathrm{E}+00$ & $8.600 \mathrm{E}+06$ \\
\hline & 138509.170 & 158715.460 & $1.841 \mathrm{E}-01$ & 6 & 6 & $1.585 \mathrm{E}-03$ & $1.549 \mathrm{E}-01$ & $4.314 \mathrm{E}+05$ \\
\hline & 138509.170 & 158215.590 & $1.796 \mathrm{E}-01$ & 6 & 4 & $2.164 \mathrm{E}-02$ & $2.169 \mathrm{E}+\infty$ & $8.411 \mathrm{E}+06$ \\
\hline \multirow[t]{5}{*}{$\mathrm{b}^{2} \mathrm{~F}^{\mathrm{e}} \rightarrow \mathrm{z}^{2} \mathrm{~F}^{\mathrm{o}}$} & 0.4584 & 0.4424 & $1.605 \mathrm{E}-02$ & 14 & 14 & $4.262 \mathrm{E}-03$ & $1.115 \mathrm{E}+01$ & $8.816 \mathrm{E}+03$ \\
\hline & 138527.980 & 140319.230 & $1.630 \mathrm{E}-02$ & 8 & 8 & $4.174 \mathrm{E}-03$ & $6.146 \mathrm{E}+00$ & $8.908 E+03$ \\
\hline & 138527.980 & 140230.100 & $1.550 \mathrm{E}-02$ & 8 & 6 & $1.470 \mathrm{E}-04$ & $2.276 \mathrm{E}-01$ & $3.782 \mathrm{E}+02$ \\
\hline & 138509.170 & 140319.230 & $1.650 \mathrm{E}-02$ & 6 & 8 & $2.087 \mathrm{E}-04$ & $2.276 \mathrm{E}-01$ & $3.422 \mathrm{E}+02$ \\
\hline & 138509.170 & 140230.100 & $1.570 \mathrm{E}-02$ & 6 & 6 & $4.010 \mathrm{E}-03$ & $4.598 \mathrm{E}+00$ & $7.940 \mathrm{E}+03$ \\
\hline \multirow[t]{5}{*}{$\mathrm{b}^{2} \mathrm{~F}^{\mathrm{e}} \rightarrow \mathrm{y}^{2} \mathrm{~F}^{\circ}$} & 0.4584 & 0.1856 & $2.728 \mathrm{E}-01$ & 14 & 14 & $1.903 \mathrm{E}-07$ & $2.929 \mathrm{E}-05$ & $1.138 \mathrm{E}+02$ \\
\hline & 138527.980 & 168473.480 & $2.728 \mathrm{E}-01$ & 8 & 8 & $1.835 \mathrm{E}-07$ & $1.614 \mathrm{E}-05$ & $1.097 \mathrm{E}+02$ \\
\hline & 138527.980 & 168443.140 & $2.726 \mathrm{E}-01$ & 8 & 6 & $6.790 \mathrm{E}-09$ & $5.978 \mathrm{E}-07$ & $5.404 \mathrm{E}+00$ \\
\hline & 138509.170 & 168473.480 & $2.730 \mathrm{E}-01$ & 6 & 8 & $9.067 \mathrm{E}-09$ & $5.978 \mathrm{E}-07$ & $4.071 \mathrm{E}+00$ \\
\hline & 138509.170 & 168443.140 & $2.728 \mathrm{E}-01$ & 6 & 6 & $1.830 \mathrm{E}-07$ & $1.208 \mathrm{E}-05$ & $1.094 \mathrm{E}+02$ \\
\hline \multirow[t]{4}{*}{$c^{2} F^{e} \rightarrow w^{2} D^{\circ}$} & 0.2955 & 0.2762 & $1.930 \mathrm{E}-02$ & 14 & 10 & $1.454 \mathrm{E}-01$ & $3.165 \mathrm{E}+02$ & $6.093 \mathrm{E}+05$ \\
\hline & 156604.170 & 158715.460 & $1.920 \mathrm{E}-02$ & 8 & 6 & $1.447 \mathrm{E}-01$ & $1.808 \mathrm{E}+02$ & $5.712 \mathrm{E}+05$ \\
\hline & 156121.700 & 158715.460 & $2.360 \mathrm{E}-02$ & 6 & 6 & $1.186 \mathrm{E}-02$ & $9.042 \mathrm{E}+\infty 0$ & $5.304 \mathrm{E}+04$ \\
\hline & 156121.700 & 158215.590 & $1.910 \mathrm{E}-02$ & 6 & 4 & $1.343 \mathrm{E}-01$ & $1.266 \mathrm{E}+02$ & $5.904 \mathrm{E}+05$ \\
\hline
\end{tabular}


Table IV. Continued

\begin{tabular}{|c|c|c|c|c|c|c|c|c|}
\hline Transition & $\begin{array}{l}E_{i} \\
\mathrm{~cm}^{-1}\end{array}$ & $\begin{array}{l}E_{f} \\
\mathrm{~cm}^{-1}\end{array}$ & $\begin{array}{l}E_{f i} \\
\mathrm{Ry}\end{array}$ & $g_{i}$ & $g_{f}$ & $f_{i f}$ & $S$ & $\begin{array}{l}A_{f i} \\
\mathrm{~s}^{-1} \\
\end{array}$ \\
\hline \multirow{5}{*}{$c^{2} F^{e} \rightarrow y^{2} F^{0}$} & 0.2955 & 0.1856 & $1.099 \mathrm{E}-01$ & 14 & 14 & $1.229 \mathrm{E}-02$ & $4.695 \mathrm{E}+\infty 0$ & $1.193 E+06$ \\
\hline & 156604.170 & 168473.480 & $1.081 \mathrm{E}-01$ & 8 & 8 & $1.165 \mathrm{E}-02$ & $2.587 \mathrm{E}+00$ & $1.094 \mathrm{E}+06$ \\
\hline & 156604.170 & 168443.140 & $1.079 \mathrm{E}-01$ & 8 & 6 & $4.308 \mathrm{E}-04$ & $9.582 \mathrm{E}-02$ & $5.371 \mathrm{E}+04$ \\
\hline & 156121.700 & 168473.480 & $1.125 \mathrm{E}-01$ & 6 & 8 & $5.989 \mathrm{E}-04$ & $9.582 \mathrm{E}-02$ & $4.566 \mathrm{E}+0.4$ \\
\hline & 156121.700 & 168443.140 & $1.123 \mathrm{E}-01$ & 6 & 6 & $1.208 \mathrm{E}-02$ & $1.936 \mathrm{E}+00$ & $1.223 \mathrm{E}+06$ \\
\hline \multirow[t]{5}{*}{$\mathrm{d}^{2} \mathrm{~F}^{\mathrm{e}} \rightarrow \mathrm{y}^{2} \mathrm{~F}^{\mathrm{o}}$} & 0.2236 & 0.1856 & $3.798 \mathrm{E}-02$ & 14 & 14 & $4.974 \mathrm{E}-02$ & $5.500 \mathrm{E}+01$ & $5.763 \mathrm{E}+05$ \\
\hline & 164337.610 & 168473.480 & $3.760 \mathrm{E}-02$ & 8 & 8 & $4.748 \mathrm{E}-02$ & $3.031 \mathrm{E}+01$ & $5.392 \mathrm{E}+05$ \\
\hline & 164337.610 & 168443.140 & $3.740 \mathrm{E}-02$ & 8 & 6 & $1.749 \mathrm{E}-03$ & $1.122 \mathrm{E}+00$ & $2.620 \mathrm{E}+04$ \\
\hline & 164232.360 & 168473.480 & $3.860 \mathrm{E}-02$ & 6 & 8 & $2.407 \mathrm{E}-03$ & $1.122 \mathrm{E}+00$ & $2.160 \mathrm{E}+04$ \\
\hline & 164232.360 & 168443.140 & $3.840 \mathrm{E}-02$ & 6 & 6 & $4.837 \mathrm{E}-02$ & $2.267 \mathrm{E}+01$ & $5.729 \mathrm{E}+05$ \\
\hline \multirow[t]{4}{*}{$\mathrm{a}^{2} \mathrm{G}^{\mathrm{e}} \rightarrow \mathrm{z}^{2} \mathrm{~F}^{\mathrm{e}}$} & 0.5622 & 0.4424 & $1.199 \mathrm{E}-01$ & 18 & 14 & $1.141 \mathrm{E}-01$ & $5.142 \mathrm{E}+01$ & $1.693 \mathrm{E}+07$ \\
\hline & 127128.350 & 140319.230 & $1.202 \mathrm{E}-01$ & 10 & 8 & $1.145 E-01$ & $2.857 \mathrm{E}+01$ & $1.660 \mathrm{E}+07$ \\
\hline & 127127.100 & 140319.230 & $1.202 \mathrm{E}-01$ & 8 & 8 & $4.088 \mathrm{E}-03$ & $8.162 \mathrm{E}-01$ & $4.744 E+05$ \\
\hline & 127127.100 & 140230.100 & $1.194 \mathrm{E}-01$ & 8 & 6 & $1.096 \mathrm{E}-01$ & $2.204 \mathrm{E}+01$ & $1.674 \mathrm{E}+07$ \\
\hline \multirow{4}{*}{$a^{2} G^{e} \rightarrow y^{2} F^{0}$} & 0.5622 & 0.1856 & $3.767 \mathrm{E}-01$ & 18 & 14 & $2.826 \mathrm{E}-03$ & $4.051 \mathrm{E}-01$ & $4.140 \mathrm{E}+06$ \\
\hline & 127128.350 & 168473.480 & $3.767 \mathrm{E}-01$ & 10 & 8 & $2.826 \mathrm{E}-03$ & $2.251 \mathrm{E}-01$ & $4.026 \mathrm{E}+06$ \\
\hline & 127127.100 & 168473.480 & $3.767 \mathrm{E}-01$ & 8 & 8 & $1.009 \mathrm{E}-04$ & $6.430 \mathrm{E}-03$ & $1.150 \mathrm{E}+05$ \\
\hline & 127127.100 & 168443.140 & $3.765 \mathrm{E}-01$ & 8 & 6 & $2.724 \mathrm{E}-03$ & $1.736 \mathrm{E}-01$ & $4.135 E+06$ \\
\hline \multirow[t]{4}{*}{$b^{2} G^{e} \rightarrow y^{2} F^{\circ}$} & 0.2241 & 0.1856 & $3.855 \mathrm{E}-02$ & 18 & 14 & $2.608 \mathrm{E}-01$ & $3.653 \mathrm{E}+02$ & $4.003 \mathrm{E}+06$ \\
\hline & 164268.790 & 168473.480 & $3.830 \mathrm{E}-02$ & 10 & 8 & $2.591 \mathrm{E}-01$ & $2.029 E+02$ & $3.816 \mathrm{E}+06$ \\
\hline & 164181.170 & 168473.480 & $3.910 \mathrm{E}-02$ & 8 & 8 & $9.446 \mathrm{E}-03$ & $5.798 \mathrm{E}+00$ & $1.160 \mathrm{E}+05$ \\
\hline & 164181.170 & 168443.140 & $3.890 \mathrm{E}-02$ & 8 & 6 & $2.537 \mathrm{E}-01$ & $1.566 \mathrm{E}+02$ & $4.112 \mathrm{E}+06$ \\
\hline \multirow[t]{3}{*}{$\mathrm{z}^{2} \mathrm{~S}^{\circ} \rightarrow \mathrm{c}^{2} \mathrm{P}^{e}$} & 0.5772 & 0.4453 & $1.319 \mathrm{E}-01$ & 2 & 6 & $9.295 \mathrm{E}-02$ & $4.228 \mathrm{E}+00$ & $4.330 \mathrm{E}+06$ \\
\hline & 125485.290 & 140016.770 & $1.324 \mathrm{E}-01$ & 2 & 4 & $6.220 \mathrm{E}-02$ & $2.819 \mathrm{E}+00$ & $4.379 E+06$ \\
\hline & 125485.290 & 139844.990 & $1.309 \mathrm{E}-01$ & 2 & 2 & $3.075 \mathrm{E}-02$ & $1.409 \mathrm{E}+00$ & $4.232 \mathrm{E}+06$ \\
\hline \multirow[t]{3}{*}{$\mathrm{z}^{2} \mathrm{~S}^{0} \rightarrow \mathrm{d}^{2} \mathrm{P}^{\mathrm{e}}$} & 0.5772 & 0.3936 & $1.836 \mathrm{E}-01$ & 2 & 6 & $4.301 \mathrm{E}-01$ & $1.406 \mathrm{E}+01$ & $3.881 \mathrm{E}+07$ \\
\hline & 125485.290 & 145505.740 & $1.824 \mathrm{E}-01$ & 2 & 4 & $2.849 \mathrm{E}-01$ & $9.372 \mathrm{E}+00$ & $3.807 \mathrm{E}+07$ \\
\hline & 125485.290 & 145877.660 & $1.858 \mathrm{E}-01$ & 2 & 2 & $1.451 \mathrm{E}-01$ & $4.686 \mathrm{E}+00$ & $4.024 \mathrm{E}+07$ \\
\hline \multirow[t]{3}{*}{$\mathrm{z}^{2} \mathrm{~S}^{0} \rightarrow \mathrm{e}^{2} \mathrm{P}^{\mathrm{c}}$} & 0.5772 & 0.3380 & $2.392 \mathrm{E}-01$ & 2 & 6 & $3.464 \mathrm{E}-01$ & $8.690 \mathrm{E}+00$ & $5.307 \mathrm{E}+07$ \\
\hline & 125485.290 & 151910.830 & $2.408 \mathrm{E}-01$ & 2 & 4 & $2.325 \mathrm{E}-01$ & $5.793 \mathrm{E}+00$ & $5.414 \mathrm{E}+07$ \\
\hline & 125485.290 & 151383.810 & $2.360 \mathrm{E}-01$ & 2 & 2 & $1.139 \mathrm{E}-01$ & $2.897 \mathrm{E}+00$ & $5.097 \mathrm{E}+07$ \\
\hline \multirow[t]{3}{*}{$\mathrm{z}^{2} \mathrm{~S}^{\circ} \rightarrow \mathrm{f}^{2} \mathrm{P}^{\mathrm{e}}$} & 0.5772 & 0.2720 & $3.052 \mathrm{E}-01$ & 2 & 6 & $9.039 \mathrm{E}-02$ & $1.777 \mathrm{E}+00$ & $2.255 \mathrm{E}+07$ \\
\hline & 125485.290 & 158828.310 & $3.039 \mathrm{E}-01$ & 2 & 4 & $6.000 \mathrm{E}-02$ & $1.185 \mathrm{E}+00$ & $2.225 \mathrm{E}+07$ \\
\hline & 125485.290 & 159283.660 & $3.080 \mathrm{E}-01$ & 2 & 2 & $3.040 E-02$ & $5.923 \mathrm{E}-01$ & $2.317 \mathrm{E}+07$ \\
\hline \multirow[t]{3}{*}{$\mathrm{z}^{2} \mathrm{~S}^{\circ} \rightarrow \mathrm{g}^{2} \mathrm{P}^{\mathrm{e}}$} & 0.5772 & 0.2064 & $3.708 \mathrm{E}-01$ & 2 & 6 & $4.962 \mathrm{E}-02$ & $8.030 \mathrm{E}-01$ & $1.827 \mathrm{E}+07$ \\
\hline & 125485.290 & 166185.470 & $3.709 \mathrm{E}-01$ & 2 & 4 & $3.309 \mathrm{E}-02$ & $5.353 \mathrm{E}-01$ & $1.828 \mathrm{E}+07$ \\
\hline & 125485.290 & 166151.610 & $3.706 \mathrm{E}-01$ & 2 & 2 & $1.653 \mathrm{E}-02$ & $2.677 \mathrm{E}-01$ & $1.824 \mathrm{E}+07$ \\
\hline \multirow[t]{3}{*}{$\mathrm{z}^{2} \mathrm{~S}^{\mathrm{a}} \rightarrow \mathrm{b}^{2} \mathrm{P}^{\mathrm{e}}$} & 0.5772 & 0.2017 & $3.754 \mathrm{E}-01$ & 2 & 6 & $3.456 \mathrm{E}-03$ & $5.523 \mathrm{E}-02$ & $1.304 \mathrm{E}+06$ \\
\hline & 125485.290 & 166862.710 & $3.771 \mathrm{E}-01$ & 2 & 4 & $2.314 \mathrm{E}-03$ & $3.682 \mathrm{E}-02$ & $1.322 E+06$ \\
\hline & 125485.290 & 166331.190 & $3.722 \mathrm{E}-01$ & 2 & 2 & $1.142 E-03$ & $1.841 E-02$ & $1.271 \mathrm{E}+06$ \\
\hline \multirow[t]{3}{*}{$\mathrm{z}^{2} \mathrm{~S}^{\circ} \rightarrow \mathrm{i}^{2} \mathrm{P}^{\mathrm{e}}$} & 0.5772 & 0.1765 & $4.007 \mathrm{E}-01$ & 2 & 6 & $7.927 \mathrm{E}-02$ & $1.187 \mathrm{E}+00$ & $3.407 \mathrm{E}+07$ \\
\hline & 125485.290 & 169340.390 & $3.996 \mathrm{E}-01$ & 2 & 4 & $5.270 \mathrm{E}-02$ & $7.913 \mathrm{E}-01$ & $3.380 \mathrm{E}+07$ \\
\hline & 125485.290 & 169687.520 & $4.028 \mathrm{E}-01$ & 2 & 2 & $2.656 \mathrm{E}-02$ & $3.956 \mathrm{E}-01$ & $3.461 \mathrm{E}+07$ \\
\hline \multirow[t]{3}{*}{$z^{2} S^{\circ} \rightarrow j^{2} P^{\bullet}$} & 0.5772 & 0.1342 & $4.430 \mathrm{E}-01$ & 2 & 6 & $1.323 \mathrm{E}-02$ & $1.792 \mathrm{E}-01$ & $6.951 E+06$ \\
\hline & 125485.290 & 174274.520 & $4.446 \mathrm{E}-01$ & 2 & 4 & $8.850 \mathrm{E}-03$ & $1.194 \mathrm{E}-01$ & $7.026 \mathrm{E}+06$ \\
\hline & 125485.290 & 173749.010 & $4.398 \mathrm{E}-01$ & 2 & 2 & $4.377 \mathrm{E}-03$ & $5.972 \mathrm{E}-02$ & $6.801 \mathrm{E}+06$ \\
\hline
\end{tabular}


Table IV. Continued

\begin{tabular}{|c|c|c|c|c|c|c|c|c|}
\hline Transition & $\begin{array}{l}E_{i} \\
\mathrm{~cm}^{-1} \\
\end{array}$ & $\begin{array}{l}E_{f} \\
\mathrm{~cm}^{-1}\end{array}$ & $\begin{array}{l}E_{f i} \\
\text { Ry }\end{array}$ & $g_{i}$ & $g_{f}$ & $f_{i f}$ & $S$ & $\begin{array}{l}A_{f_{i}} \\
\mathrm{~s}^{-1}\end{array}$ \\
\hline \multirow[t]{3}{*}{$\mathrm{z}^{2} \mathrm{P}^{\circ} \rightarrow \mathrm{a}^{2} \mathrm{~S}^{\bullet}$} & 1.4969 & 0.6291 & $8.678 \mathrm{E}-01$ & 6 & 2 & $8.039 \mathrm{E}-03$ & $1.667 \mathrm{E}-01$ & $1.459 \mathrm{E}+08$ \\
\hline & 24571.540 & 119783.770 & $8.677 \mathrm{E}-01$ & 4 & 2 & $8.038 \mathrm{E}-03$ & $1.112 \mathrm{E}-01$ & $9.721 E+07$ \\
\hline & 24524.830 & 119783.770 & $8.681 \mathrm{E}-01$ & 2 & 2 & $8.042 \mathrm{E}-03$ & $5.558 \mathrm{E}-02$ & $4.868 E+07$ \\
\hline \multirow[t]{3}{*}{$z^{2} P^{0} \rightarrow b^{2} S^{e}$} & 1.4969 & 0.4784 & $1.019 E+00$ & 6 & 2 & $1.188 \mathrm{E}-01$ & $2.099 \mathrm{E}+00$ & $2.970 \mathrm{E}+09$ \\
\hline & 24571.540 & 136328.790 & $1.018 \mathrm{E}+00$ & 4 & 2 & $1.188 \mathrm{E}-01$ & $1.400 \mathrm{E}+00$ & $1.979 E+09$ \\
\hline & 24524.830 & 136328.790 & $1.019 \mathrm{E}+00$ & 2 & 2 & $1.188 \mathrm{E}-01$ & $6.998 \mathrm{E}-01$ & $9.907 \mathrm{E}+08$ \\
\hline \multirow[t]{3}{*}{$z^{2} P^{\circ} \rightarrow c^{2} S^{e}$} & 1.4969 & 0.3387 & $1.158 \mathrm{E}+00$ & 6 & 2 & $1.393 \mathrm{E}-01$ & $2.165 \mathrm{E}+00$ & $4.504 \mathrm{E}+09$ \\
\hline & 24571.540 & 151651.720 & $1.158 \mathrm{E}+00$ & 4 & 2 & $1.393 \mathrm{E}-01$ & $1.444 \mathrm{E}+00$ & $3.002 \mathrm{E}+09$ \\
\hline & 24524.830 & 151651.720 & $1.159 \mathrm{E}+\infty 0$ & 2 & 2 & $1.394 \mathrm{E}-01$ & $7.218 \mathrm{E}-01$ & $1.502 \mathrm{E}+09$ \\
\hline \multirow[t]{3}{*}{$z^{2} \mathbf{P}^{o} \rightarrow d^{2} S^{e}$} & 1.4969 & 0.1797 & $1.317 \mathrm{E}+00$ & 6 & 2 & $7.520 \mathrm{E}-02$ & $1.028 \mathrm{E}+00$ & $3.144 \mathrm{E}+09$ \\
\hline & 24571.540 & 169109.540 & $1.317 \mathrm{E}+\infty 0$ & 4 & 2 & $7.519 \mathrm{E}-02$ & $6.850 \mathrm{E}-01$ & $2.095 \mathrm{E}+09$ \\
\hline & 24524.830 & 169109.540 & $1.318 \mathrm{E}+\infty 0$ & 2 & 2 & $7.521 \mathrm{E}-02$ & $3.425 \mathrm{E}-01$ & $1.049 E+09$ \\
\hline \multirow[t]{5}{*}{$\mathrm{z}^{2} \mathrm{P}^{\circ} \rightarrow \mathrm{a}^{2} \mathrm{P}^{\circ}$} & 1.4969 & 0.7571 & $7.399 \mathrm{E}-01$ & 6 & 6 & $1.990 \mathrm{E}-03$ & $4.841 \mathrm{E}-02$ & $8.749 E+06$ \\
\hline & 24571.540 & 105599.060 & $7.384 \mathrm{E}-01$ & 4 & 4 & $1.655 \mathrm{E}-03$ & $2.689 \mathrm{E}-02$ & $7.246 \mathrm{E}+06$ \\
\hline & 24571.540 & 106044.240 & $7.424 \mathrm{E}-01$ & 4 & 2 & $3.328 \mathrm{E}-04$ & $5.379 \mathrm{E}-03$ & $2.947 \mathrm{E}+06$ \\
\hline & 24524.830 & 105599.060 & $7.388 \mathrm{E}-01$ & 2 & 4 & $6.623 \mathrm{E}-04$ & $5.379 \mathrm{E}-03$ & $1.452 \mathrm{E}+06$ \\
\hline & 24524.830 & 106044.240 & $7.429 \mathrm{E}-01$ & 2 & 2 & $1.332 \mathrm{E}-03$ & $1.076 \mathrm{E}-02$ & $5.903 E+06$ \\
\hline \multirow[t]{5}{*}{$\mathrm{z}^{2} \mathrm{P}^{\mathrm{o}} \rightarrow \mathrm{b}^{2} \mathrm{P}^{\mathrm{e}}$} & 1.4969 & 0.6884 & $8.086 \mathrm{E}-01$ & 6 & 6 & $9.361 \mathrm{E}-02$ & $2.084 \mathrm{E}+00$ & $4.916 \mathrm{E}+08$ \\
\hline & 24571.540 & 113461.540 & $8.100 \mathrm{E}-01$ & 4 & 4 & $7.814 \mathrm{E}-02$ & $1.158 \mathrm{E}+\infty 0$ & $4.118 \mathrm{E}+08$ \\
\hline & 24571.540 & 112937.570 & $8.053 \mathrm{E}-01$ & 4 & 2 & $1.554 \mathrm{E}-02$ & $2.315 \mathrm{E}-01$ & $1.619 \mathrm{E}+08$ \\
\hline & 24524.830 & 113461.540 & $8.104 \mathrm{E}-01$ & 2 & 4 & $3.127 \mathrm{E}-02$ & $2.315 \mathrm{E}-01$ & $8.249 E+07$ \\
\hline & 24524.830 & 112937.570 & $8.057 \mathrm{E}-01$ & 2 & 2 & $6.218 \mathrm{E}-02$ & $4.631 \mathrm{E}-01$ & $3.242 \mathrm{E}+08$ \\
\hline \multirow[t]{5}{*}{$\mathrm{z}^{2} \mathrm{P}^{o} \rightarrow \mathrm{c}^{2} \mathrm{P}^{\mathrm{e}}$} & 1.4969 & 0.4453 & $1.052 \mathrm{E}+00$ & 6 & 6 & $3.081 \mathrm{E}-01$ & $5.274 \mathrm{E}+00$ & $2.737 \mathrm{E}+09$ \\
\hline & 24571.540 & 140016.770 & $1.052 \mathrm{E}+00$ & 4 & 4 & $2.569 \mathrm{E}-01$ & $2.930 \mathrm{E}+00$ & $2.283 E+09$ \\
\hline & 24571.540 & 139844.990 & $1.050 \mathrm{E}+00$ & 4 & 2 & $5.130 \mathrm{E}-02$ & $5.860 \mathrm{E}-01$ & $9.094 E+08$ \\
\hline & 24524.830 & 140016.770 & $1.052 \mathrm{E}+\infty 0$ & 2 & 4 & $1.028 \mathrm{E}-01$ & $5.860 \mathrm{E}-01$ & $4.572 \mathrm{E}+08$ \\
\hline & 24524.830 & 139844.990 & $1.051 \mathrm{E}+00$ & 2 & 2 & $2.053 \mathrm{E}-01$ & $1.172 \mathrm{E}+00$ & $1.821 \mathrm{E}+09$ \\
\hline \multirow{5}{*}{$z^{2} P^{o} \rightarrow d^{2} P^{e}$} & 1.4969 & 0.3936 & $1.103 E+00$ & 6 & 6 & $7.068 \mathrm{E}-02$ & $1.153 E+00$ & $6.910 \mathrm{E}+08$ \\
\hline & 24571.540 & 145505.740 & $1.102 \mathrm{E}+\infty$ & 4 & 4 & $5.883 \mathrm{E}-02$ & $6.406 \mathrm{E}-01$ & $5.738 \mathrm{E}+08$ \\
\hline & 24571.540 & 145877.660 & $1.105 \mathrm{E}+\infty$ & 4 & 2 & $1.180 \mathrm{E}-02$ & $1.281 \mathrm{E}-01$ & $2.317 \mathrm{E}+08$ \\
\hline & 24524.830 & 145505.740 & $1.102 \mathrm{E}+\infty 0$ & 2 & 4 & $2.354 \mathrm{E}-02$ & $1.281 \mathrm{E}-01$ & $1.149 \mathrm{E}+08$ \\
\hline & 24524.830 & 145877.660 & $1.106 \mathrm{E}+00$ & 2 & 2 & $4.722 \mathrm{E}-02$ & $2.562 \mathrm{E}-01$ & $4.638 \mathrm{E}+08$ \\
\hline \multirow[t]{5}{*}{$z^{2} P^{o} \rightarrow e^{2} P^{e}$} & 1.4969 & 0.3380 & $1.159 E+00$ & 6 & 6 & $2.622 \mathrm{E}-02$ & $4.072 \mathrm{E}-01$ & $2.828 \mathrm{E}+08$ \\
\hline & 24571.540 & 151910.830 & $1.160 \mathrm{E}+00$ & 4 & 4 & $2.187 \mathrm{E}-02$ & $2.262 \mathrm{E}-01$ & $2.366 \mathrm{E}+08$ \\
\hline & 24571.540 & 151383.810 & $1.156 \mathrm{E}+00$ & 4 & 2 & $4.357 \mathrm{E}-03$ & $4.524 \mathrm{E}-02$ & $9.346 \mathrm{E}+07$ \\
\hline & 24524.830 & 151910.830 & $1.161 \mathrm{E}+00$ & 2 & 4 & $8.753 \mathrm{E}-03$ & $4.524 \mathrm{E}-02$ & $4.737 \mathrm{E}+07$ \\
\hline & 24524.830 & 151383.810 & $1.156 \mathrm{E}+00$ & 2 & 2 & $1.743 \mathrm{E}-02$ & $9.048 \mathrm{E}-02$ & $1.871 \mathrm{E}+08$ \\
\hline \multirow[t]{5}{*}{$\mathrm{z}^{2} \mathrm{P}^{\mathrm{o}} \rightarrow \mathrm{f}^{2} \mathrm{P}^{\mathrm{e}}$} & 1.4969 & 0.2720 & $1.225 \mathrm{E}+00$ & 6 & 6 & $2.552 \mathrm{E}-01$ & $3.750 \mathrm{E}+00$ & $3.076 \mathrm{E}+09$ \\
\hline & 24571.540 & 158828.310 & $1.223 \mathrm{E}+00$ & 4 & 4 & $2.124 \mathrm{E}-01$ & $2.083 E+00$ & $2.554 \mathrm{E}+09$ \\
\hline & 24571.540 & 159283.660 & $1.228 \mathrm{E}+00$ & 4 & 2 & $4.263 \mathrm{E}-02$ & $4.167 \mathrm{E}-01$ & $1.032 \mathrm{E}+09$ \\
\hline & 24524.830 & 158828.310 & $1.224 \mathrm{E}+\infty 0$ & 2 & 4 & $8.500 \mathrm{E}-02$ & $4.167 \mathrm{E}-01$ & $5.114 \mathrm{E}+08$ \\
\hline & 24524.830 & 159283.660 & $1.228 \mathrm{E}+00$ & 2 & 2 & $1.706 \mathrm{E}-01$ & $8.334 \mathrm{E}-01$ & $2.066 \mathrm{E}+09$ \\
\hline \multirow[t]{5}{*}{$z^{2} P^{\circ} \rightarrow g^{2} P^{e}$} & 1.4969 & 0.2064 & $1.291 \mathrm{E}+00$ & 6 & 6 & $1.418 \mathrm{E}-02$ & $1.978 \mathrm{E}-01$ & $1.897 \mathrm{E}+08$ \\
\hline & 24571.540 & 166185.470 & $1.290 \mathrm{E}+00$ & 4 & 4 & $1.182 \mathrm{E}-02$ & $1.099 \mathrm{E}-01$ & $1.581 \mathrm{E}+08$ \\
\hline & 24571.540 & 166151.610 & $1.290 \mathrm{E}+00$ & 4 & 2 & $2.363 \mathrm{E}-03$ & $2.197 \mathrm{E}-02$ & $6.318 \mathrm{E}+07$ \\
\hline & 24524.830 & 166185.470 & $1.291 \mathrm{E}+\infty$ & 2 & 4 & $4.728 \mathrm{E}-03$ & $2.197 \mathrm{E}-02$ & $3.164 \mathrm{E}+07$ \\
\hline & 24524.830 & 166151.610 & $1.291 \mathrm{E}+00$ & 2 & 2 & $9.454 \mathrm{E}-03$ & $4.395 \mathrm{E}-02$ & $1.265 \mathrm{E}+08$ \\
\hline \multirow[t]{5}{*}{$z^{2} P^{o} \rightarrow h^{2} P^{e}$} & 1.4969 & 0.2017 & $1.295 \mathrm{E}+00$ & 6 & 6 & $1.902 \mathrm{E}-03$ & $2.644 \mathrm{E}-02$ & $2.563 \mathrm{E}+07$ \\
\hline & 24571.540 & 166862.710 & $1.297 \mathrm{E}+00$ & 4 & 4 & $1.587 \mathrm{E}-03$ & $1.469 \mathrm{E}-02$ & $2.143 E+07$ \\
\hline & 24571.540 & 166331.190 & $1.292 \mathrm{E}+00$ & 4 & 2 & $3.162 \mathrm{E}-04$ & $2.937 \mathrm{E}-03$ & $8.477 \mathrm{E}+06$ \\
\hline & 24524.830 & 166862.710 & $1.297 \mathrm{E}+00$ & 2 & 4 & $6.350 \mathrm{E}-04$ & $2.937 \mathrm{E}-03$ & $4.291 \mathrm{E}+06$ \\
\hline & 24524.830 & 166331.190 & $1.292 \mathrm{E}+\infty 0$ & 2 & 2 & $1.265 \mathrm{E}-03$ & $5.875 \mathrm{E}-03$ & $1.697 \mathrm{E}+07$ \\
\hline
\end{tabular}


Table IV. Continued

\begin{tabular}{|c|c|c|c|c|c|c|c|c|}
\hline Transition & $\begin{array}{l}E_{i} \\
\mathrm{~cm}^{-1}\end{array}$ & $\begin{array}{l}E_{f} \\
\mathrm{~cm}^{-1}\end{array}$ & $\begin{array}{l}E_{f i} \\
\mathrm{Ry}\end{array}$ & $g_{i}$ & $g_{f}$ & $f_{i f}$ & $s$ & $\begin{array}{l}A_{f i} \\
\mathrm{~s}^{-1} \\
\end{array}$ \\
\hline \multirow[t]{5}{*}{$\mathrm{z}^{2} \mathrm{P}^{\mathrm{o}} \rightarrow \mathrm{i}^{2} \mathrm{P}^{\mathrm{e}}$} & 1.4969 & 0.1765 & $1.320 \mathrm{E}+00$ & 6 & 6 & $1.478 \mathrm{E}-01$ & $2.015 \mathrm{E}+00$ & $2.070 \mathrm{E}+09$ \\
\hline & 24571.540 & 169340.390 & $1.319 \mathrm{E}+00$ & 4 & 4 & $1.231 \mathrm{E}-01$ & $1.119 E+00$ & $1.720 E+09$ \\
\hline & 24571.540 & 169687.520 & $1.322 \mathrm{E}+00$ & 4 & 2 & $2.467 \mathrm{E}-02$ & $2.239 \mathrm{E}-01$ & $6.930 \mathrm{E}+08$ \\
\hline & 24524.830 & 169340.390 & $1.320 \mathrm{E}+00$ & 2 & 4 & $4.924 \mathrm{E}-02$ & $2.239 \mathrm{E}-01$ & $3.443 \mathrm{E}+08$ \\
\hline & 24524.830 & 169687.520 & $1.323 \mathrm{E}+00$ & 2 & 2 & $9.871 \mathrm{E}-02$ & $4.477 \mathrm{E}-01$ & $1.387 \mathrm{E}+09$ \\
\hline \multirow{5}{*}{$\mathrm{z}^{2} \mathrm{P}^{\circ} \rightarrow \mathrm{j}^{2} \mathrm{P}^{\mathrm{e}}$} & 1.4969 & 0.1342 & $1.363 \mathrm{E}+00$ & 6 & 6 & $7.781 \mathrm{E}-03$ & $1.028 \mathrm{E}-01$ & $1.161 \mathrm{E}+08$ \\
\hline & 24571.540 & 174274.520 & $1.364 \mathrm{E}+00$ & 4 & 4 & $6.491 \mathrm{E}-03$ & $5.710 \mathrm{E}-02$ & $9.703 \mathrm{E}+07$ \\
\hline & 24571.540 & 173749.010 & $1.359 \mathrm{E}+00$ & 4 & 2 & $1.294 \mathrm{E}-03$ & $1.142 \mathrm{E}-02$ & $3.841 E+07$ \\
\hline & 24524.830 & 174274.520 & $1.365 \mathrm{E}+00$ & 2 & 4 & $2.597 \mathrm{E}-03$ & $1.142 \mathrm{E}-02$ & $1.942 \mathrm{E}+07$ \\
\hline & 24524.830 & 173749.010 & $1.360 \mathrm{E}+00$ & 2 & 2 & $5.176 \mathrm{E}-03$ & $2.284 \mathrm{E}-02$ & $7.688 \mathrm{E}+07$ \\
\hline \multirow{4}{*}{$z^{2} P^{\circ} \rightarrow a^{2} D^{e}$} & 1.4969 & 0.8285 & $6.684 \mathrm{E}-01$ & 6 & 10 & $3.266 \mathrm{E}-03$ & $8.796 \mathrm{E}-02$ & $7.034 \mathrm{E}+06$ \\
\hline & 24571.540 & 97918.860 & $6.684 \mathrm{E}-01$ & 4 & 6 & $2.940 \mathrm{E}-03$ & $5.278 \mathrm{E}-02$ & $7.032 \mathrm{E}+06$ \\
\hline & 24571.540 & 97890.740 & $6.681 \mathrm{E}-01$ & 4 & 4 & $3.265 \mathrm{E}-04$ & $5.864 \mathrm{E}-03$ & $1.171 \mathrm{E}+06$ \\
\hline & 24524.830 & 97890.740 & $6.686 \mathrm{E}-01$ & 2 & 4 & $3.267 \mathrm{E}-03$ & $2.932 \mathrm{E}-02$ & $5.865 \mathrm{E}+06$ \\
\hline \multirow[t]{4}{*}{$\mathrm{z}^{2} \mathrm{P}^{\circ} \rightarrow \mathrm{b}^{2} \mathrm{D}^{\mathrm{e}}$} & 1.4969 & 0.6132 & $8.837 \mathrm{E}-01$ & 6 & 10 & $4.219 \mathrm{E}-02$ & $8.594 \mathrm{E}-01$ & $1.588 \mathrm{E}+08$ \\
\hline & 24571.540 & 121530.020 & $8.836 \mathrm{E}-01$ & 4 & 6 & $3.797 \mathrm{E}-02$ & $5.157 \mathrm{E}-01$ & $1.587 \mathrm{E}+08$ \\
\hline & 24571.540 & 121528.720 & $8.836 \mathrm{E}-01$ & 4 & 4 & $4.219 \mathrm{E}-03$ & $5.730 \mathrm{E}-02$ & $2.646 \mathrm{E}+07$ \\
\hline & 24524.830 & 121528.720 & $8.840 \mathrm{E}-01$ & 2 & 4 & $4.221 \mathrm{E}-02$ & $2.865 \mathrm{E}-01$ & $1.325 \mathrm{E}+08$ \\
\hline \multirow[t]{4}{*}{$\mathrm{z}^{2} \mathrm{P}^{\circ} \rightarrow \mathrm{c}^{2} \mathrm{D}^{\mathrm{e}}$} & 1.4969 & 0.5029 & $9.940 \mathrm{E}-01$ & 6 & 10 & $3.049 \mathrm{E}-01$ & $5.521 \mathrm{E}+\infty 0$ & $1.452 \mathrm{E}+09$ \\
\hline & 24571.540 & 133814.840 & $9.955 \mathrm{E}-01$ & 4 & 6 & $2.748 \mathrm{E}-01$ & $3.313 \mathrm{E}+00$ & $1.458 \mathrm{E}+09$ \\
\hline & 24571.540 & 133360.860 & $9.914 \mathrm{E}-01$ & 4 & 4 & $3.041 \mathrm{E}-02$ & $3.681 \mathrm{E}-01$ & $2.401 E+08$ \\
\hline & 24524.830 & 133360.860 & $9.918 \mathrm{E}-01$ & 2 & 4 & $3.042 \mathrm{E}-01$ & $1.840 \mathrm{E}+00$ & $1.202 \mathrm{E}+09$ \\
\hline \multirow[t]{4}{*}{$\mathrm{z}^{2} \mathrm{P}^{\circ} \rightarrow \mathrm{d}^{2} \mathrm{D}^{\mathrm{e}}$} & 1.4969 & 0.4079 & $1.089 \mathrm{E}+00$ & 6 & 10 & $1.281 \mathrm{E}-02$ & $2.118 \mathrm{E}-01$ & $7.323 E+07$ \\
\hline & 24571.540 & 144009.420 & $1.088 \mathrm{E}+00$ & 4 & 6 & $1.152 \mathrm{E}-02$ & $1.271 \mathrm{E}-01$ & $7.310 \mathrm{E}+07$ \\
\hline & 24571.540 & 144142.160 & $1.090 \mathrm{E}+00$ & 4 & 4 & $1.282 \mathrm{E}-03$ & $1.412 \mathrm{E}-02$ & $1.222 \mathrm{E}+07$ \\
\hline & 24524.830 & 144142.160 & $1.090 \mathrm{E}+00$ & 2 & 4 & $1.282 \mathrm{E}-02$ & $7.059 \mathrm{E}-02$ & $6.119 \mathrm{E}+07$ \\
\hline \multirow[t]{4}{*}{$\mathrm{z}^{2} \mathrm{P}^{\circ} \rightarrow \mathrm{e}^{2} \mathrm{D}^{e}$} & 1.4969 & 0.3639 & $1.133 E+\infty 0$ & 6 & 10 & $4.900 \mathrm{E}-01$ & $7.785 \mathrm{E}+00$ & $3.032 \mathrm{E}+09$ \\
\hline & 24571.540 & 148886.570 & $1.133 \mathrm{E}+\infty 0$ & 4 & 6 & $4.410 \mathrm{E}-01$ & $4.671 \mathrm{E}+\infty 0$ & $3.031 E+09$ \\
\hline & 24571.540 & 148900.910 & $1.133 \mathrm{E}+\infty 0$ & 4 & 4 & $4.900 \mathrm{E}-02$ & $5.190 \mathrm{E}-01$ & $5.052 \mathrm{E}+08$ \\
\hline & 24524.830 & 148900.910 & $1.133 \mathrm{E}+\infty 0$ & 2 & 4 & $4.902 \mathrm{E}-01$ & $2.595 \mathrm{E}+\infty 0$ & $2.529 \mathrm{E}+09$ \\
\hline \multirow[t]{4}{*}{$\mathrm{z}^{2} \mathrm{P}^{\circ} \rightarrow \mathrm{f}^{2} \mathrm{D}^{\bullet}$} & 1.4969 & 0.2673 & $1.230 \mathrm{E}+\infty$ & 6 & 10 & $3.879 \mathrm{E}-01$ & $5.679 \mathrm{E}+00$ & $2.827 \mathrm{E}+09$ \\
\hline & 24571.540 & 159492.830 & $1.229 \mathrm{E}+\infty 0$ & 4 & 6 & $3.491 \mathrm{E}-01$ & $3.407 \mathrm{E}+00$ & $2.826 \mathrm{E}+09$ \\
\hline & 24571.540 & 159495.940 & $1.229 E+00$ & 4 & 4 & $3.879 \mathrm{E}-02$ & $3.786 \mathrm{E}-01$ & $4.710 E+08$ \\
\hline & 24524.830 & 159495.940 & $1.230 \mathrm{E}+00$ & 2 & 4 & $3.880 \mathrm{E}-01$ & $1.893 \mathrm{E}+00$ & $2.357 \mathrm{E}+09$ \\
\hline \multirow[t]{4}{*}{$z^{2} P^{0} \rightarrow g^{2} D^{e}$} & 1.4969 & 0.2469 & $1.250 \mathrm{E}+\infty 0$ & 6 & 10 & $1.915 \mathrm{E}-02$ & $2.758 \mathrm{E}-01$ & $1.443 \mathrm{E}+08$ \\
\hline & 24571.540 & 161733.100 & $1.250 \mathrm{E}+00$ & 4 & 6 & $1.724 \mathrm{E}-02$ & $1.655 \mathrm{E}-01$ & $1.442 E+08$ \\
\hline & 24571.540 & 161737.990 & $1.250 \mathrm{E}+\infty 0$ & 4 & 4 & $1.915 \mathrm{E}-03$ & $1.839 \mathrm{E}-02$ & $2.404 \mathrm{E}+07$ \\
\hline & 24524.830 & 161737.990 & $1.250 \mathrm{E}+\infty 0$ & 2 & 4 & $1.916 \mathrm{E}-02$ & $9.194 \mathrm{E}-02$ & $1.203 E+08$ \\
\hline \multirow[t]{4}{*}{$\mathrm{z}^{2} \mathrm{P}^{\circ} \rightarrow \mathrm{h}^{2} \mathrm{D}^{\mathrm{e}}$} & 1.4969 & 0.1944 & $1.303 E+\infty$ & 6 & 10 & $7.514 \mathrm{E}-02$ & $1.038 \mathrm{E}+00$ & $6.144 \mathrm{E}+08$ \\
\hline & 24571.540 & 167506.390 & $1.302 \mathrm{E}+00$ & 4 & 6 & $6.763 \mathrm{E}-02$ & $6.231 \mathrm{E}-01$ & $6.144 \mathrm{E}+08$ \\
\hline & 24571.540 & 167472.420 & $1.302 \mathrm{E}+00$ & 4 & 4 & $7.512 \mathrm{E}-03$ & $6.923 \mathrm{E}-02$ & $1.023 \mathrm{E}+08$ \\
\hline & 24524.830 & 167472.420 & $1.303 \mathrm{E}+00$ & 2 & 4 & $7.515 \mathrm{E}-02$ & $3.461 \mathrm{E}-01$ & $5.121 \mathrm{E}+08$ \\
\hline \multirow[t]{4}{*}{$\mathrm{z}^{2} \mathrm{P}^{\circ} \rightarrow \mathrm{i}^{2} \mathrm{D}^{\mathrm{e}}$} & 1.4969 & 0.1717 & $1.325 \mathrm{E}+\infty 0$ & 6 & 10 & $1.533 \mathrm{E}-01$ & $2.082 \mathrm{E}+00$ & $1.297 \mathrm{E}+09$ \\
\hline & 24571.540 & 170015.230 & $1.325 \mathrm{E}+\infty$ & 4 & 6 & $1.380 \mathrm{E}-01$ & $1.249 \mathrm{E}+00$ & $1.298 \mathrm{E}+09$ \\
\hline & 24571.540 & 169922.270 & $1.324 \mathrm{E}+\infty$ & 4 & 4 & $1.532 \mathrm{E}-02$ & $1.388 \mathrm{E}-01$ & $2.158 \mathrm{E}+08$ \\
\hline & 24524.830 & 169922.270 & $1.325 \mathrm{E}+\infty 0$ & 2 & 4 & $1.532 \mathrm{E}-01$ & $6.939 \mathrm{E}-01$ & $1.080 \mathrm{E}+09$ \\
\hline \multirow[t]{4}{*}{$\mathrm{z}^{2} \mathrm{P}^{\mathrm{o}} \rightarrow \mathrm{j}^{2} \mathrm{D}^{\mathrm{e}}$} & 1.4969 & 0.1071 & $1.390 \mathrm{E}+00$ & 6 & 10 & $6.527 \mathrm{E}-03$ & $8.454 \mathrm{E}-02$ & $6.077 \mathrm{E}+07$ \\
\hline & 24571.540 & 177076.280 & $1.390 \mathrm{E}+00$ & 4 & 6 & $5.874 E-03$ & $5.072 \mathrm{E}-02$ & $6.075 \mathrm{E}+07$ \\
\hline & 24571.540 & 177077.020 & $1.390 \mathrm{E}+00$ & 4 & 4 & $6.527 \mathrm{E}-04$ & $5.636 \mathrm{E}-03$ & $1.012 \mathrm{E}+07$ \\
\hline & 24524.830 & 177077.020 & $1.390 \mathrm{E}+00$ & 2 & 4 & $6.529 \mathrm{E}-03$ & $2.818 \mathrm{E}-02$ & $5.067 \mathrm{E}+07$ \\
\hline
\end{tabular}


Table IV. Continued

\begin{tabular}{|c|c|c|c|c|c|c|c|c|}
\hline Transition & $\begin{array}{l}E_{i} \\
\mathrm{~cm}^{-1}\end{array}$ & $\begin{array}{l}E_{f} \\
\mathrm{~cm}^{-1}\end{array}$ & $\begin{array}{l}E_{f i} \\
\mathrm{Ry}\end{array}$ & $g_{i}$ & $g_{f}$ & $f_{i f}$ & $S$ & $\begin{array}{l}A_{f i} \\
\mathrm{~s}^{-1} \\
\end{array}$ \\
\hline \multirow[t]{4}{*}{$\mathrm{z}^{2} \mathrm{P}^{0} \rightarrow \mathrm{k}^{2} \mathrm{D}^{\mathrm{e}}$} & 1.4969 & 0.0381 & $1.459 \mathrm{E}+00$ & 6 & 10 & $1.648 \mathrm{E}-02$ & $2.033 \mathrm{E}-01$ & $1.690 \mathrm{E}+08$ \\
\hline & 24571.540 & 184642.760 & $1.459 \mathrm{E}+00$ & 4 & 6 & $1.483 \mathrm{E}-02$ & $1.220 \mathrm{E}-01$ & $1.689 \mathrm{E}+08$ \\
\hline & 24571.540 & 184642.760 & $1.459 \mathrm{E}+00$ & 4 & 4 & $1.647 \mathrm{E}-03$ & $1.355 \mathrm{E}-02$ & $2.816 \mathrm{E}+07$ \\
\hline & 24524.830 & 184642.760 & $1.459 \mathrm{E}+00$ & 2 & 4 & $1.648 \mathrm{E}-02$ & $6.776 \mathrm{E}-02$ & $1.409 \mathrm{E}+08$ \\
\hline \multirow[t]{3}{*}{$y^{2} P^{o} b^{2} S^{e}$} & 0.5055 & 0.4784 & $2.709 \mathrm{E}-02$ & 6 & 2 & $9.425 \mathrm{E}-04$ & $6.263 \mathrm{E}-01$ & $1.667 \mathrm{E}+04$ \\
\hline & 133399.970 & 136328.790 & $2.670 \mathrm{E}-02$ & 4 & 2 & $9.290 \mathrm{E}-04$ & $4.175 \mathrm{E}-01$ & $1.064 \mathrm{E}+04$ \\
\hline & 133268.680 & 136328.790 & $2.790 \mathrm{E}-02$ & 2 & 2 & $9.708 \mathrm{E}-04$ & $2.088 \mathrm{E}-01$ & $6.070 \mathrm{E}+03$ \\
\hline \multirow[t]{3}{*}{$\mathrm{y}^{2} \mathrm{P}^{\circ} \rightarrow \mathrm{c}^{2} \mathrm{~S}^{\mathrm{c}}$} & 0.5055 & 0.3387 & $1.667 \mathrm{E}-01$ & 6 & 2 & $6.022 \mathrm{E}-03$ & $6.501 \mathrm{E}-01$ & $4.033 E+06$ \\
\hline & 133399.970 & 151651.720 & $1.664 \mathrm{E}-01$ & 4 & 2 & $6.010 \mathrm{E}-03$ & $4.334 \mathrm{E}-01$ & $2.673 \mathrm{E}+06$ \\
\hline & 133268.680 & 151651.720 & $1.676 \mathrm{E}-01$ & 2 & 2 & $6.053 E-03$ & $2.167 \mathrm{E}-01$ & $1.366 \mathrm{E}+06$ \\
\hline \multirow[t]{3}{*}{$y^{2} P^{o} \rightarrow d^{2} S^{e}$} & 0.5055 & 0.1797 & $3.258 \mathrm{E}-01$ & 6 & 2 & $5.689 \mathrm{E}-03$ & $3.143 \mathrm{E}-01$ & $1.455 \mathrm{E}+07$ \\
\hline & 133399.970 & 169109.540 & $3.254 \mathrm{E}-01$ & 4 & 2 & $5.682 \mathrm{E}-03$ & $2.095 \mathrm{E}-01$ & $9.664 \mathrm{E}+06$ \\
\hline & 133268.680 & 169109.540 & $3.266 \mathrm{E}-01$ & 2 & 2 & $5.703 E-03$ & $1.048 \mathrm{E}-01$ & $4.886 \mathrm{E}+06$ \\
\hline \multirow[t]{5}{*}{$\mathrm{y}^{2} \mathrm{P}^{\circ} \rightarrow \mathrm{c}^{2} \mathrm{P}^{e}$} & 0.5055 & 0.4453 & $6.017 \mathrm{E}-02$ & 6 & 6 & $9.800 \mathrm{E}-03$ & $2.932 \mathrm{E}+00$ & $2.850 \mathrm{E}+05$ \\
\hline & 133399.970 & 140016.770 & $6.030 \mathrm{E}-02$ & 4 & 4 & $8.184 \mathrm{E}-03$ & $1.629 \mathrm{E}+00$ & $2.390 \mathrm{E}+05$ \\
\hline & 133399.970 & 139844.990 & $5.880 \mathrm{E}-02$ & 4 & 2 & $1.596 \mathrm{E}-03$ & $3.257 \mathrm{E}-01$ & $8.865 \mathrm{E}+04$ \\
\hline & 133268.680 & 140016.770 & $6.150 \mathrm{E}-02$ & 2 & 4 & $3.339 \mathrm{E}-03$ & $3.257 \mathrm{E}-01$ & $5.072 \mathrm{E}+04$ \\
\hline & 133268.680 & 139844.990 & $6.000 \mathrm{E}-02$ & 2 & 2 & $6.515 \mathrm{E}-03$ & $6.515 \mathrm{E}-01$ & $1.884 \mathrm{E}+05$ \\
\hline \multirow[t]{5}{*}{$\mathrm{y}^{2} \mathrm{P}^{\circ} \rightarrow \mathrm{d}^{2} \mathrm{P}^{\mathrm{e}}$} & 0.5055 & 0.3936 & $1.118 \mathrm{E}-01$ & 6 & 6 & $1.973 \mathrm{E}-01$ & $3.176 \mathrm{E}+01$ & $1.983 E+07$ \\
\hline & 133399.970 & 145505.740 & $1.103 \mathrm{E}-01$ & 4 & 4 & $1.622 \mathrm{E}-01$ & $1.764 \mathrm{E}+01$ & $1.585 \mathrm{E}+07$ \\
\hline & 133399.970 & 145877.660 & $1.137 \mathrm{E}-01$ & 4 & 2 & $3.343 \mathrm{E}-02$ & $3.528 \mathrm{E}+00$ & $6.943 \mathrm{E}+06$ \\
\hline & 133268.680 & 145505.740 & $1.115 \mathrm{E}-01$ & 2 & 4 & $6.557 \mathrm{E}-02$ & $3.528 \mathrm{E}+00$ & $3.274 \mathrm{E}+06$ \\
\hline & 133268.680 & 145877.660 & $1.149 \mathrm{E}-01$ & 2 & 2 & $1.351 \mathrm{E}-01$ & $7.057 \mathrm{E}+00$ & $1.433 \mathrm{E}+07$ \\
\hline \multirow[t]{5}{*}{$\mathrm{y}^{2} \mathrm{P}^{0} \rightarrow \mathrm{e}^{2} \mathrm{P}^{\mathrm{e}}$} & 0.5055 & 0.3380 & $1.675 \mathrm{E}-01$ & 6 & 6 & $1.409 \mathrm{E}-01$ & $1.515 \mathrm{E}+01$ & $3.176 \mathrm{E}+07$ \\
\hline & 133399.970 & 151910.830 & $1.687 \mathrm{E}-01$ & 4 & 4 & $1.183 E-01$ & $8.416 \mathrm{E}+00$ & $2.705 \mathrm{E}+07$ \\
\hline & 133399.970 & 151383.810 & $1.639 \mathrm{E}-01$ & 4 & 2 & $2.299 \mathrm{E}-02$ & $1.683 \mathrm{E}+00$ & $9.921 \mathrm{E}+06$ \\
\hline & 133268.680 & 151910.830 & $1.699 \mathrm{E}-01$ & 2 & 4 & $4.766 \mathrm{E}-02$ & $1.683 \mathrm{E}+00$ & $5.525 \mathrm{E}+06$ \\
\hline & 133268.680 & 151383.810 & $1.651 \mathrm{E}-01$ & 2 & 2 & $9.263 \mathrm{E}-02$ & $3.366 \mathrm{E}+\infty 0$ & $2.028 \mathrm{E}+07$ \\
\hline \multirow[t]{5}{*}{$y^{2} P^{\circ} \rightarrow f^{2} P^{e}$} & 0.5055 & 0.2720 & $2.335 \mathrm{E}-01$ & 6 & 6 & $6.665 \mathrm{E}-02$ & $5.138 \mathrm{E}+00$ & $2.919 \mathrm{E}+07$ \\
\hline & 133399.970 & 158828.310 & $2.318 \mathrm{E}-01$ & 4 & 4 & $5.514 \mathrm{E}-02$ & $2.854 \mathrm{E}+00$ & $2.380 \mathrm{E}+07$ \\
\hline & 133399.970 & 159283.660 & $2.359 \mathrm{E}-01$ & 4 & 2 & $1.122 \mathrm{E}-02$ & $5.709 \mathrm{E}-01$ & $1.003 \mathrm{E}+07$ \\
\hline & 133268.680 & 158828.310 & $2.330 \mathrm{E}-01$ & 2 & 4 & $2.217 \mathrm{E}-02$ & $5.709 \mathrm{E}-01$ & $4.834 \mathrm{E}+06$ \\
\hline & 133268.680 & 159283.660 & $2.371 \mathrm{E}-01$ & 2 & 2 & $4.512 \mathrm{E}-02$ & $1.142 \mathrm{E}+00$ & $2.037 \mathrm{E}+07$ \\
\hline \multirow[t]{5}{*}{$y^{2} P^{\circ} \rightarrow g^{2} P^{0}$} & 0.5055 & 0.2064 & $2.991 \mathrm{E}-01$ & 6 & 6 & $3.082 \mathrm{E}-04$ & $1.855 \mathrm{E}-02$ & $2.214 \mathrm{E}+05$ \\
\hline & 133399.970 & 166185.470 & $2.988 \mathrm{E}-01$ & 4 & 4 & $2.566 \mathrm{E}-04$ & $1.031 \mathrm{E}-02$ & $1.840 \mathrm{E}+05$ \\
\hline & 133399.970 & 166151.610 & $2.985 \mathrm{E}-01$ & 4 & 2 & $5.127 \mathrm{E}-05$ & $2.061 \mathrm{E}-03$ & $7.338 \mathrm{E}+04$ \\
\hline & 133268.680 & 166185.470 & $3.000 \mathrm{E}-01$ & 2 & 4 & $1.031 \mathrm{E}-04$ & $2.061 \mathrm{E}-03$ & $3.725 E+04$ \\
\hline & 133268.680 & 166151.610 & $2.997 \mathrm{E}-01$ & 2 & 2 & $2.059 \mathrm{E}-04$ & $4.122 \mathrm{E}-03$ & $1.485 \mathrm{E}+05$ \\
\hline \multirow[t]{5}{*}{$y^{2} P^{o} \rightarrow h^{2} P^{c}$} & 0.5055 & 0.2017 & $3.037 \mathrm{E}-01$ & 6 & 6 & $4.896 \mathrm{E}-02$ & $2.902 \mathrm{E}+00$ & $3.628 \mathrm{E}+07$ \\
\hline & 133399.970 & 166862.710 & $3.050 \mathrm{E}-01$ & 4 & 4 & $4.097 \mathrm{E}-02$ & $1.612 \mathrm{E}+00$ & $3.061 \mathrm{E}+07$ \\
\hline & 133399.970 & 166331.190 & $3.001 \mathrm{E}-01$ & 4 & 2 & $8.063 \mathrm{E}-03$ & $3.224 \mathrm{E}-01$ & $1.166 \mathrm{E}+07$ \\
\hline & 133268.680 & 166862.710 & $3.062 \mathrm{E}-01$ & 2 & 4 & $1.645 \mathrm{E}-02$ & $3.224 \mathrm{E}-01$ & $6.195 \mathrm{E}+06$ \\
\hline & 133268.680 & 166331.190 & $3.013 E-01$ & 2 & 2 & $3.238 \mathrm{E}-02$ & $6.448 \mathrm{E}-01$ & $2.361 \mathrm{E}+07$ \\
\hline \multirow[t]{5}{*}{$\mathrm{y}^{2} \mathbf{P}^{o} \rightarrow \mathrm{i}^{2} \mathbf{P}^{\mathrm{e}}$} & 0.5055 & 0.1765 & $3.290 \mathrm{E}-01$ & 6 & 6 & $1.632 \mathrm{E}-02$ & $8.927 \mathrm{E}-01$ & $1.418 \mathrm{E}+07$ \\
\hline & 133399.970 & 169340.390 & $3.275 \mathrm{E}-01$ & 4 & 4 & $1.354 \mathrm{E}-02$ & $4.960 \mathrm{E}-01$ & $1.166 \mathrm{E}+07$ \\
\hline & 133399.970 & 169687.520 & $3.307 \mathrm{E}-01$ & 4 & 2 & $2.734 \mathrm{E}-03$ & $9.919 \mathrm{E}-02$ & $4.803 E+06$ \\
\hline & 133268.680 & 169340.390 & $3.287 \mathrm{E}-01$ & 2 & 4 & $5.434 \mathrm{E}-03$ & $9.919 \mathrm{E}-02$ & $2.358 \mathrm{E}+06$ \\
\hline & 133268.680 & 169687.520 & $3.319 \mathrm{E}-01$ & 2 & 2 & $1.097 \mathrm{E}-02$ & $1.984 \mathrm{E}-01$ & $9.710 \mathrm{E}+06$ \\
\hline
\end{tabular}


Table IV. Continued

\begin{tabular}{|c|c|c|c|c|c|c|c|c|}
\hline Transition & $\begin{array}{l}E_{i} \\
\mathrm{~cm}^{-1}\end{array}$ & $\begin{array}{l}E_{f} \\
\mathrm{~cm}^{-1}\end{array}$ & $\begin{array}{l}E_{f i} \\
\mathrm{Ry}\end{array}$ & $g_{i}$ & $g_{f}$ & $f_{i f}$ & $s$ & $\begin{array}{l}A_{f i} \\
\mathrm{~s}^{-1} \\
\end{array}$ \\
\hline \multirow[t]{5}{*}{$y^{2} P^{o} \rightarrow j^{2} P^{e}$} & 0.5055 & 0.1342 & $3.713 \mathrm{E}-01$ & 6 & 6 & $7.287 \mathrm{E}-03$ & $3.533 \mathrm{E}-01$ & $8.068 \mathrm{E}+06$ \\
\hline & 133399.970 & 174274.520 & $3.725 \mathrm{E}-01$ & 4 & 4 & $6.092 \mathrm{E}-03$ & $1.963 \mathrm{E}-01$ & $6.790 \mathrm{E}+06$ \\
\hline & 133399.970 & 173749.010 & $3.677 \mathrm{E}-01$ & 4 & 2 & $1.203 \mathrm{E}-03$ & $3.925 \mathrm{E}-02$ & $2.612 E+06$ \\
\hline & 133268.680 & 174274.520 & $3.737 \mathrm{E}-01$ & 2 & 4 & $2.445 \mathrm{E}-03$ & $3.925 \mathrm{E}-02$ & $1.371 \mathrm{E}+06$ \\
\hline & 133268.680 & 173749.010 & $3.689 \mathrm{E}-01$ & 2 & 2 & $4.827 \mathrm{E}-03$ & $7.851 E-02$ & $5.276 \mathrm{E}+06$ \\
\hline \multirow[t]{4}{*}{$y^{2} P^{o} \rightarrow c^{2} D^{0}$} & 0.5055 & 0.5029 & $2.525 E-03$ & 6 & 10 & $5.186 \mathrm{E}-03$ & $3.698 \mathrm{E}+01$ & $1.593 \mathrm{E}+02$ \\
\hline & 133399.970 & 133814.840 & $3.800 \mathrm{E}-03$ & 4 & 6 & $7.025 \mathrm{E}-03$ & $2.219 \mathrm{E}+01$ & $5.432 \mathrm{E}+02$ \\
\hline & 133360.860 & 133399.970 & $3.000 \mathrm{E}-04$ & 4 & 4 & $6.163 \mathrm{E}-05$ & $2.465 \mathrm{E}+00$ & $4.455 \mathrm{E}-02$ \\
\hline & 133268.680 & 133360.860 & $9.000 \mathrm{E}-04$ & 2 & 4 & $1.849 \mathrm{E}-03$ & $1.233 \mathrm{E}+01$ & $6.014 \mathrm{E}+00$ \\
\hline \multirow{4}{*}{$\mathrm{y}^{2} \mathrm{P}^{\circ} \rightarrow \mathrm{d}^{2} \mathrm{D}^{\circ}$} & 0.5055 & 0.4079 & $9.756 \mathrm{E}-02$ & 6 & 10 & $5.659 \mathrm{E}-02$ & $1.044 \mathrm{E}+01$ & $2.596 \mathrm{E}+06$ \\
\hline & 133399.970 & 144009.420 & $9.670 \mathrm{E}-02$ & 4 & 6 & $5.048 \mathrm{E}-02$ & $6.264 \mathrm{E}+00$ & $2.528 E+06$ \\
\hline & 133399.970 & 144142.160 & $9.790 \mathrm{E}-02$ & 4 & 4 & $5.678 \mathrm{E}-03$ & $6.960 \mathrm{E}-01$ & $4.372 E+05$ \\
\hline & 133268.680 & 144142.160 & $9.910 \mathrm{E}-02$ & 2 & 4 & $5.748 \mathrm{E}-02$ & $3.480 \mathrm{E}+00$ & $2.267 \mathrm{E}+06$ \\
\hline \multirow[t]{4}{*}{$y^{2} P^{o} \rightarrow e^{2} D^{e}$} & 0.5055 & 0.3639 & $1.416 \mathrm{E}-01$ & 6 & 10 & $1.022 \mathrm{E}-01$ & $1.300 \mathrm{E}+01$ & $9.876 \mathrm{E}+06$ \\
\hline & 133399.970 & 148886.570 & $1.412 E-01$ & 4 & 6 & $9.177 \mathrm{E}-02$ & $7.799 \mathrm{E}+\infty 0$ & $9.797 \mathrm{E}+06$ \\
\hline & 133399.970 & 148900.910 & $1.413 \mathrm{E}-01$ & 4 & 4 & $1.020 \mathrm{E}-02$ & $8.666 \mathrm{E}-01$ & $1.636 \mathrm{E}+06$ \\
\hline & 133268.680 & 148900.910 & $1.425 \mathrm{E}-01$ & 2 & 4 & $1.029 \mathrm{E}-01$ & $4.333 \mathrm{E}+00$ & $8.392 \mathrm{E}+06$ \\
\hline \multirow[t]{4}{*}{$y^{2} P^{o} \rightarrow f^{2} D^{e}$} & 0.5055 & 0.2673 & $2.382 \mathrm{E}-01$ & 6 & 10 & $2.963 \mathrm{E}-01$ & $2.239 \mathrm{E}+01$ & $8.102 E+07$ \\
\hline & 133399.970 & 159492.830 & $2.378 \mathrm{E}-01$ & 4 & 6 & $2.663 \mathrm{E}-01$ & $1.344 \mathrm{E}+01$ & $8.063 \mathrm{E}+07$ \\
\hline & 133399.970 & 159495.940 & $2.378 \mathrm{E}-01$ & 4 & 4 & $2.958 \mathrm{E}-02$ & $1.493 \mathrm{E}+00$ & $1.344 \mathrm{E}+07$ \\
\hline & 133268.680 & 159495.940 & $2.390 \mathrm{E}-01$ & 2 & 4 & $2.973 \mathrm{E}-01$ & $7.464 \mathrm{E}+00$ & $6.821 \mathrm{E}+07$ \\
\hline \multirow[t]{4}{*}{$y^{2} P^{o} \rightarrow g^{2} D^{e}$} & 0.5055 & 0.2469 & $2.586 \mathrm{E}-01$ & 6 & 10 & $5.660 \mathrm{E}-03$ & $3.940 \mathrm{E}-01$ & $1.824 \mathrm{E}+06$ \\
\hline & 133399.970 & 161733.100 & $2.582 \mathrm{E}-01$ & 4 & 6 & $5.086 \mathrm{E}-03$ & $2.364 \mathrm{E}-01$ & $1.816 \mathrm{E}+06$ \\
\hline & 133399.970 & 161737.990 & $2.583 \mathrm{E}-01$ & 4 & 4 & $5.653 \mathrm{E}-04$ & $2.626 \mathrm{E}-02$ & $3.030 \mathrm{E}+05$ \\
\hline & 133268.680 & 161737.990 & $2.595 \mathrm{E}-01$ & 2 & 4 & $5.680 \mathrm{E}-03$ & $1.313 \mathrm{E}-01$ & $1.536 \mathrm{E}+06$ \\
\hline \multirow[t]{4}{*}{$y^{2} P^{\circ} \rightarrow h^{2} D^{e}$} & 0.5055 & 0.1944 & $3.111 \mathrm{E}-01$ & 6 & 10 & $3.266 \mathrm{E}-02$ & $1.890 \mathrm{E}+00$ & $1.523 \mathrm{E}+07$ \\
\hline & 133399.970 & 167506.390 & $3.108 \mathrm{E}-01$ & 4 & 6 & $2.937 \mathrm{E}-02$ & $1.134 \mathrm{E}+00$ & $1.519 \mathrm{E}+07$ \\
\hline & 133399.970 & 167472.420 & $3.105 \mathrm{E}-01$ & 4 & 4 & $3.260 \mathrm{E}-03$ & $1.260 \mathrm{E}-01$ & $2.525 \mathrm{E}+06$ \\
\hline & 133268.680 & 167472.420 & $3.117 \mathrm{E}-01$ & 2 & 4 & $3.273 \mathrm{E}-02$ & $6.300 \mathrm{E}-01$ & $1.277 \mathrm{E}+07$ \\
\hline \multirow[t]{4}{*}{$\mathrm{y}^{2} \mathrm{P}^{\circ} \rightarrow \mathrm{i}^{2} \mathrm{D}^{\mathrm{c}}$} & 0.5055 & 0.1717 & $3.337 \mathrm{E}-01$ & 6 & 10 & $7.654 \mathrm{E}-02$ & $4.129 \mathrm{E}+00$ & $4.108 \mathrm{E}+07$ \\
\hline & 133399.970 & 170015.230 & $3.337 \mathrm{E}-01$ & 4 & 6 & $6.888 \mathrm{E}-02$ & $2.477 \mathrm{E}+00$ & $4.108 \mathrm{E}+07$ \\
\hline & 133399.970 & 169922.270 & $3.328 \mathrm{E}-01$ & 4 & 4 & $7.633 E-03$ & $2.752 \mathrm{E}-01$ & $6.791 \mathrm{E}+06$ \\
\hline & 133268.680 & 169922.270 & $3.340 \mathrm{E}-01$ & 2 & 4 & $7.661 \mathrm{E}-02$ & $1.376 \mathrm{E}+\infty 0$ & $3.432 \mathrm{E}+07$ \\
\hline \multirow[t]{4}{*}{$y^{2} P^{\circ} \rightarrow j^{2} D^{\circ}$} & 0.5055 & 0.1071 & $3.984 \mathrm{E}-01$ & 6 & 10 & $1.816 \mathrm{E}-03$ & $8.205 \mathrm{E}-02$ & $1.389 \mathrm{E}+06$ \\
\hline & 133399.970 & 177076.280 & $3.980 \mathrm{E}-01$ & 4 & 6 & $1.633 \mathrm{E}-03$ & $4.923 \mathrm{E}-02$ & $1.385 \mathrm{E}+06$ \\
\hline & 133399.970 & 177077.020 & $3.980 \mathrm{E}-01$ & 4 & 4 & $1.814 \mathrm{E}-04$ & $5.470 \mathrm{E}-03$ & $2.308 \mathrm{E}+05$ \\
\hline & 133268.680 & 177077.020 & $3.992 \mathrm{E}-01$ & 2 & 4 & $1.820 E-03$ & $2.735 \mathrm{E}-02$ & $1.165 \mathrm{E}+06$ \\
\hline \multirow[t]{4}{*}{$y^{2} P^{0} \rightarrow k^{2} D^{e}$} & 0.5055 & 0.0381 & $4.674 \mathrm{E}-01$ & 6 & 10 & $4.334 \mathrm{E}-03$ & $1.669 \mathrm{E}-01$ & $4.562 \mathrm{E}+06$ \\
\hline & 133399.970 & 184642.760 & $4.670 \mathrm{E}-01$ & 4 & 6 & $3.898 E-03$ & $1.002 \mathrm{E}-01$ & $4.552 \mathrm{E}+06$ \\
\hline & 133399.970 & 184642.760 & $4.670 \mathrm{E}-01$ & 4 & 4 & $4.331 E-04$ & $1.113 E-02$ & $7.586 \mathrm{E}+05$ \\
\hline & 133268.680 & 184642.760 & $4.682 \mathrm{E}-01$ & 2 & 4 & $4.342 E-03$ & $5.564 \mathrm{E}-02$ & $3.822 \mathrm{E}+06$ \\
\hline \multirow[t]{3}{*}{$\mathrm{x}^{2} \mathrm{P}^{\mathrm{o}} \rightarrow \mathrm{c}^{2} \mathrm{~S}^{\mathrm{e}}$} & 0.4123 & 0.3387 & $7.357 \mathrm{E}-02$ & 6 & 2 & $4.242 \mathrm{E}-02$ & $1.038 \mathrm{E}+01$ & $5.532 \mathrm{E}+06$ \\
\hline & 143623.560 & 151651.720 & $7.320 \mathrm{E}-02$ & 4 & 2 & $4.221 \mathrm{E}-02$ & $6.919 E+00$ & $3.633 \mathrm{E}+06$ \\
\hline & 143488.950 & 151651.720 & $7.440 \mathrm{E}-02$ & 2 & 2 & $4.290 \mathrm{E}-02$ & $3.460 \mathrm{E}+\infty 0$ & $1.907 \mathrm{E}+06$ \\
\hline \multirow[t]{3}{*}{$\mathrm{x}^{2} \mathbf{P}^{\circ} \rightarrow \mathrm{d}^{2} \mathbf{S}^{e}$} & 0.4123 & 0.1797 & $2.327 \mathrm{E}-01$ & 6 & 2 & $9.744 \mathrm{E}-02$ & $7.539 \mathrm{E}+\infty 0$ & $1.271 \mathrm{E}+08$ \\
\hline & 143623.560 & 169109.540 & $2.322 \mathrm{E}-01$ & 4 & 2 & $9.725 \mathrm{E}-02$ & $5.026 \mathrm{E}+00$ & $8.424 \mathrm{E}+07$ \\
\hline & 143488.950 & 169109.540 & $2.334 E-01$ & 2 & 2 & $9.776 \mathrm{E}-02$ & $2.513 \mathrm{E}+00$ & $4.277 \mathrm{E}+07$ \\
\hline
\end{tabular}


Table IV. Continued

\begin{tabular}{|c|c|c|c|c|c|c|c|c|}
\hline Transition & $\begin{array}{l}E_{i} \\
\mathrm{~cm}^{-1}\end{array}$ & $\begin{array}{l}E_{f} \\
\mathrm{~cm}^{-1}\end{array}$ & $\begin{array}{l}E_{f i} \\
\mathrm{Ry}\end{array}$ & $g_{i}$ & $g_{f}$ & $f_{i f}$ & $S$ & $\begin{array}{l}A_{f_{i}} \\
\mathrm{~s}^{-1}\end{array}$ \\
\hline \multirow{5}{*}{$\mathrm{x}^{2} \mathrm{P}^{\circ} \rightarrow \mathrm{d}^{2} \mathrm{P}^{\mathrm{e}}$} & 0.4123 & 0.3936 & $1.869 \mathrm{E}-02$ & 6 & 6 & $8.777 \mathrm{E}-05$ & $8.453 \mathrm{E}-02$ & $2.463 \mathrm{E}+02$ \\
\hline & 143623.560 & 145505.740 & $1.710 \mathrm{E}-02$ & 4 & 4 & $6.692 \mathrm{E}-05$ & $4.696 \mathrm{E}-02$ & $1.572 \mathrm{E}+02$ \\
\hline & 143623.560 & 145877.660 & $2.050 \mathrm{E}-02$ & 4 & 2 & $1.604 \mathrm{E}-05$ & $9.392 \mathrm{E}-03$ & $1.083 E+02$ \\
\hline & 143488.950 & 145505.740 & $1.830 \mathrm{E}-02$ & 2 & 4 & $2.865 \mathrm{E}-05$ & $9.392 \mathrm{E}-03$ & $3.853 E+01$ \\
\hline & 143488.950 & 145877.660 & $2.170 \mathrm{E}-02$ & 2 & 2 & $6.793 \mathrm{E}-05$ & $1.878 \mathrm{E}-02$ & $2.569 E+02$ \\
\hline \multirow[t]{5}{*}{$x^{2} P^{o} \rightarrow e^{2} P^{e}$} & 0.4123 & 0.3380 & $7.433 E-02$ & 6 & 6 & $2.522 \mathrm{E}-02$ & $6.109 \mathrm{E}+00$ & $1.119 E+06$ \\
\hline & 143623.560 & 151910.830 & $7.550 \mathrm{E}-02$ & 4 & 4 & $2.135 \mathrm{E}-02$ & $3.394 \mathrm{E}+00$ & $9.776 \mathrm{E}+05$ \\
\hline & 143623.560 & 151383.810 & $7.070 \mathrm{E}-02$ & 4 & 2 & $3.999 \mathrm{E}-03$ & $6.787 \mathrm{E}-01$ & $3.211 \mathrm{E}+05$ \\
\hline & 143488.950 & 151910.830 & $7.670 \mathrm{E}-02$ & 2 & 4 & $8.676 \mathrm{E}-03$ & $6.787 \mathrm{E}-01$ & $2.050 \mathrm{E}+05$ \\
\hline & 143488.950 & 151383.810 & $7.190 \mathrm{E}-02$ & 2 & 2 & $1.627 \mathrm{E}-02$ & $1.357 \mathrm{E}+\infty 0$ & $6.755 \mathrm{E}+05$ \\
\hline \multirow{5}{*}{$\mathrm{x}^{2} \mathrm{P}^{\circ} \rightarrow \mathrm{f}^{2} \mathrm{P}^{e}$} & 0.4123 & 0.2720 & $1.403 \mathrm{E}-01$ & 6 & 6 & $3.757 \mathrm{E}-03$ & $4.818 \mathrm{E}-01$ & $5.944 \mathrm{E}+05$ \\
\hline & 143623.560 & 158828.310 & $1.386 \mathrm{E}-01$ & 4 & 4 & $3.092 \mathrm{E}-03$ & $2.677 \mathrm{E}-01$ & $4.770 \mathrm{E}+05$ \\
\hline & 143623.560 & 159283.660 & $1.427 \mathrm{E}-01$ & 4 & 2 & $6.366 \mathrm{E}-04$ & $5.354 \mathrm{E}-02$ & $2.083 E+05$ \\
\hline & 143488.950 & 158828.310 & $1.398 \mathrm{E}-01$ & 2 & 4 & $1.247 \mathrm{E}-03$ & $5.354 \mathrm{E}-02$ & $9.791 E+04$ \\
\hline & 143488.950 & 159283.660 & $1.439 \mathrm{E}-01$ & 2 & 2 & $2.568 \mathrm{E}-03$ & $1.071 \mathrm{E}-01$ & $4.271 E+05$ \\
\hline \multirow[t]{5}{*}{$x^{2} P^{\circ} \rightarrow g^{2} P^{e}$} & 0.4123 & 0.2064 & $2.059 \mathrm{E}-01$ & 6 & 6 & $1.639 \mathrm{E}-01$ & $1.433 \mathrm{E}+01$ & $5.583 \mathrm{E}+07$ \\
\hline & 143623.560 & 166185.470 & $2.056 \mathrm{E}-01$ & 4 & 4 & $1.364 \mathrm{E}-01$ & $7.962 \mathrm{E}+00$ & $4.632 \mathrm{E}+07$ \\
\hline & 143623.560 & 166151.610 & $2.053 \mathrm{E}-01$ & 4 & 2 & $2.724 \mathrm{E}-02$ & $1.592 \mathrm{E}+00$ & $1.845 \mathrm{E}+07$ \\
\hline & 143488.950 & 166185.470 & $2.068 \mathrm{E}-01$ & 2 & 4 & $5.488 \mathrm{E}-02$ & $1.592 \mathrm{E}+00$ & $9.426 \mathrm{E}+06$ \\
\hline & 143488.950 & 166151.610 & $2.065 \mathrm{E}-01$ & 2 & 2 & $1.096 \mathrm{E}-01$ & $3.185 E+\infty 0$ & $3.754 \mathrm{E}+07$ \\
\hline \multirow{5}{*}{$x^{2} P^{\circ} \rightarrow h^{2} P^{e}$} & 0.4123 & 0.2017 & $2.106 \mathrm{E}-01$ & 6 & 6 & $1.563 \mathrm{E}-01$ & $1.336 \mathrm{E}+01$ & $5.565 \mathrm{E}+07$ \\
\hline & 143623.560 & 166862.710 & $2.118 \mathrm{E}-01$ & 4 & 4 & $1.310 \mathrm{E}-01$ & $7.421 E+00$ & $4.720 \mathrm{E}+07$ \\
\hline & 143623.560 & 166331.190 & $2.069 \mathrm{E}-01$ & 4 & 2 & $2.559 \mathrm{E}-02$ & $1.484 \mathrm{E}+\infty 0$ & $1.760 \mathrm{E}+07$ \\
\hline & 143488.950 & 166862.710 & $2.130 E-01$ & 2 & 4 & $5.269 \mathrm{E}-02$ & $1.484 \mathrm{E}+\infty 0$ & $9.601 E+06$ \\
\hline & 143488.950 & 166331.190 & $2.081 E-01$ & 2 & 2 & $1.030 \mathrm{E}-01$ & $2.968 \mathrm{E}+00$ & $3.581 E+07$ \\
\hline \multirow[t]{5}{*}{$\mathrm{x}^{2} \mathrm{P}^{\mathrm{o}} \rightarrow \mathrm{i}^{2} \mathrm{P}^{\mathrm{e}}$} & 0.4123 & 0.1765 & $2.358 \mathrm{E}-01$ & 6 & 6 & $4.651 \mathrm{E}-02$ & $3.550 \mathrm{E}+00$ & $2.078 \mathrm{E}+07$ \\
\hline & 143623.560 & 169340.390 & $2.343 E-01$ & 4 & 4 & $3.851 \mathrm{E}-02$ & $1.972 \mathrm{E}+\infty 0$ & $1.698 \mathrm{E}+07$ \\
\hline & 143623.560 & 169687.520 & $2.375 \mathrm{E}-01$ & 4 & 2 & $7.808 \mathrm{E}-03$ & $3.945 \mathrm{E}-01$ & $7.075 E+06$ \\
\hline & 143488.950 & 169340.390 & $2.355 \mathrm{E}-01$ & 2 & 4 & $1.548 \mathrm{E}-02$ & $3.945 \mathrm{E}-01$ & $3.449 \mathrm{E}+06$ \\
\hline & 143488.950 & 169687.520 & $2.387 \mathrm{E}-01$ & 2 & 2 & $3.139 \mathrm{E}-02$ & $7.890 \mathrm{E}-01$ & $1.437 \mathrm{E}+07$ \\
\hline \multirow[t]{5}{*}{$x^{2} P^{\circ} \rightarrow j^{2} P^{e}$} & 0.4123 & 0.1342 & $2.781 \mathrm{E}-01$ & 6 & 6 & $1.564 \mathrm{E}-04$ & $1.012 \mathrm{E}-02$ & $9.717 \mathrm{E}+04$ \\
\hline & 143623.560 & 174274.520 & $2.793 \mathrm{E}-01$ & 4 & 4 & $1.309 \mathrm{E}-04$ & $5.623 \mathrm{E}-03$ & $8.201 E+04$ \\
\hline & 143623.560 & 173749.010 & $2.745 \mathrm{E}-01$ & 4 & 2 & $2.573 \mathrm{E}-05$ & $1.125 \mathrm{E}-03$ & $3.114 \mathrm{E}+04$ \\
\hline & 143488.950 & 174274.520 & $2.805 \mathrm{E}-01$ & 2 & 4 & $5.258 \mathrm{E}-05$ & $1.125 \mathrm{E}-03$ & $1.661 E+04$ \\
\hline & 143488.950 & 173749.010 & $2.757 \mathrm{E}-01$ & 2 & 2 & $1.034 \mathrm{E}-04$ & $2.249 \mathrm{E}-03$ & $6.310 \mathrm{E}+04$ \\
\hline \multirow[t]{4}{*}{$\mathrm{x}^{2} \mathrm{P}^{\circ} \rightarrow \mathrm{d}^{2} \mathrm{D}^{\bullet}$} & 0.4123 & 0.4079 & $4.409 \mathrm{E}-03$ & 6 & 10 & $3.103 E-03$ & $1.267 \mathrm{E}+01$ & $2.907 \mathrm{E}+02$ \\
\hline & 143623.560 & 144009.420 & $3.500 \mathrm{E}-03$ & 4 & 6 & $2.217 \mathrm{E}-03$ & $7.600 \mathrm{E}+00$ & $1.454 \mathrm{E}+02$ \\
\hline & 143623.560 & 144142.160 & $4.700 \mathrm{E}-03$ & 4 & 4 & $3.307 \mathrm{E}-04$ & $8.445 \mathrm{E}-01$ & $5.869 \mathrm{E}+01$ \\
\hline & 143488.950 & 144142.160 & $5.900 \mathrm{E}-03$ & 2 & 4 & $4.152 E-03$ & $4.222 \mathrm{E}+00$ & $5.804 \mathrm{E}+02$ \\
\hline \multirow[t]{4}{*}{$\mathrm{x}^{2} \mathrm{P}^{\circ} \rightarrow \mathrm{e}^{2} \mathrm{D}^{\circ}$} & 0.4123 & 0.3639 & $4.842 \mathrm{E}-02$ & 6 & 10 & $6.550 \mathrm{E}-02$ & $2.435 \mathrm{E}+01$ & $7.401 \mathrm{E}+05$ \\
\hline & 143623.560 & 148886.570 & $4.800 \mathrm{E}-02$ & 4 & 6 & $5.843 \mathrm{E}-02$ & $1.461 \mathrm{E}+01$ & $7.209 \mathrm{E}+05$ \\
\hline & 143623.560 & 148900.910 & $4.810 \mathrm{E}-02$ & 4 & 4 & $6.506 \mathrm{E}-03$ & $1.623 \mathrm{E}+00$ & $1.209 \mathrm{E}+05$ \\
\hline & 143488.950 & 148900.910 & $4.930 \mathrm{E}-02$ & 2 & 4 & $6.668 \mathrm{E}-02$ & $8.116 \mathrm{E}+00$ & $6.509 \mathrm{E}+05$ \\
\hline \multirow[t]{4}{*}{$x^{2} P^{o} \rightarrow f^{2} D^{e}$} & 0.4123 & 0.2673 & $1.450 \mathrm{E}-01$ & 6 & 10 & $1.557 \mathrm{E}-02$ & $1.932 \mathrm{E}+\infty$ & $1.578 \mathrm{E}+06$ \\
\hline & 143623.560 & 159492.830 & $1.446 \mathrm{E}-01$ & 4 & 6 & $1.397 \mathrm{E}-02$ & $1.159 \mathrm{E}+\infty$ & $1.564 \mathrm{E}+06$ \\
\hline & 143623.560 & 159495.940 & $1.446 \mathrm{E}-01$ & 4 & 4 & $1.552 \mathrm{E}-03$ & $1.288 \mathrm{E}-01$ & $2.607 \mathrm{E}+05$ \\
\hline & 143488.950 & 159495.940 & $1.458 \mathrm{E}-01$ & 2 & 4 & $1.565 \mathrm{E}-02$ & $6.441 E-01$ & $1.336 \mathrm{E}+06$ \\
\hline \multirow[t]{4}{*}{$x^{2} P^{o} \rightarrow g^{2} D^{e}$} & 0.4123 & 0.2469 & $1.655 \mathrm{E}-01$ & 6 & 10 & $1.861 E-01$ & $2.025 \mathrm{E}+01$ & $2.456 \mathrm{E}+07$ \\
\hline & 143623.560 & 161733.100 & $1.650 \mathrm{E}-01$ & 4 & 6 & $1.671 \mathrm{E}-01$ & $1.215 \mathrm{E}+01$ & $2.436 \mathrm{E}+07$ \\
\hline & 143623.560 & 161737.990 & $1.651 \mathrm{E}-01$ & 4 & 4 & $1.857 \mathrm{E}-02$ & $1.350 \mathrm{E}+00$ & $4.067 \mathrm{E}+06$ \\
\hline & 143488.950 & 161737.990 & $1.663 \mathrm{E}-01$ & 2 & 4 & $1.871 \mathrm{E}-01$ & $6.750 \mathrm{E}+00$ & $2.078 \mathrm{E}+07$ \\
\hline
\end{tabular}


Table IV. Continued

\begin{tabular}{|c|c|c|c|c|c|c|c|c|}
\hline Transition & $\begin{array}{l}E_{i} \\
\mathrm{~cm}^{-1}\end{array}$ & $\begin{array}{l}E_{f} \\
\mathrm{~cm}^{-1}\end{array}$ & $\begin{array}{l}E_{f i} \\
\mathrm{Ry}\end{array}$ & $g_{i}$ & $g_{f}$ & $f_{i f}$ & $s$ & $\begin{array}{l}A_{f i} \\
\mathrm{~s}^{-1} \\
\end{array}$ \\
\hline \multirow[t]{4}{*}{$\mathrm{x}^{2} \mathrm{P}^{\circ} \rightarrow \mathrm{h}^{2} \mathrm{D}^{\mathrm{e}}$} & 0.4123 & 0.1944 & $2.179 \mathrm{E}-01$ & 6 & 10 & $2.021 \mathrm{E}-01$ & $1.670 \mathrm{E}+01$ & $4.626 \mathrm{E}+07$ \\
\hline & 143623.560 & 167506.390 & $2.176 \mathrm{E}-01$ & 4 & 6 & $1.817 \mathrm{E}-01$ & $1.002 \mathrm{E}+01$ & $4.606 \mathrm{E}+07$ \\
\hline & 143623.560 & 167472.420 & $2.173 E-01$ & 4 & 4 & $2.016 \mathrm{E}-02$ & $1.113 \mathrm{E}+00$ & $7.645 \mathrm{E}+06$ \\
\hline & 143488.950 & 167472.420 & $2.185 \mathrm{E}-01$ & 2 & 4 & $2.027 \mathrm{E}-01$ & $5.566 \mathrm{E}+\infty 0$ & $3.886 \mathrm{E}+07$ \\
\hline \multirow[t]{4}{*}{$\mathrm{x}^{2} \mathrm{P}^{\circ} \rightarrow \mathrm{i}^{2} \mathrm{D}^{e}$} & 0.4123 & 0.1717 & $2.406 \mathrm{E}-01$ & 6 & 10 & $2.954 \mathrm{E}-02$ & $2.210 \mathrm{E}+00$ & $8.239 E+06$ \\
\hline & 143623.560 & 170015.230 & $2.405 \mathrm{E}-01$ & 4 & 6 & $2.658 \mathrm{E}-02$ & $1.326 \mathrm{E}+00$ & $8.232 \mathrm{E}+06$ \\
\hline & 143623.560 & 169922.270 & $2.396 \mathrm{E}-01$ & 4 & 4 & $2.942 \mathrm{E}-03$ & $1.474 \mathrm{E}-01$ & $1.357 E+06$ \\
\hline & 143488.950 & 169922.270 & $2.408 \mathrm{E}-01$ & 2 & 4 & $2.957 \mathrm{E}-02$ & $7.368 \mathrm{E}-01$ & $6.886 \mathrm{E}+06$ \\
\hline \multirow[t]{4}{*}{$x^{2} P^{o} \rightarrow j^{2} D^{e}$} & 0.4123 & 0.1071 & $3.053 \mathrm{E}-01$ & 6 & 10 & $2.104 \mathrm{E}-02$ & $1.241 \mathrm{E}+00$ & $9.450 \mathrm{E}+06$ \\
\hline & 143623.560 & 177076.280 & $3.048 \mathrm{E}-01$ & 4 & 6 & $1.891 E-02$ & $7.445 \mathrm{E}-01$ & $9.408 \mathrm{E}+06$ \\
\hline & 143623.560 & 177077.020 & $3.048 \mathrm{E}-01$ & 4 & 4 & $2.101 E-03$ & $8.272 \mathrm{E}-02$ & $1.568 \mathrm{E}+06$ \\
\hline & 143488.950 & 177077.020 & $3.060 \mathrm{E}-01$ & 2 & 4 & $2.109 E-02$ & $4.136 \mathrm{E}-01$ & $7.933 E+06$ \\
\hline \multirow{4}{*}{$\mathrm{x}^{2} \mathrm{P}^{\mathrm{o}} \rightarrow \mathrm{K}^{2} \mathrm{D}^{\mathrm{e}}$} & 0.4123 & 0.0381 & $3.742 \mathrm{E}-01$ & 6 & 10 & $2.833 E-04$ & $1.363 \mathrm{E}-02$ & $1.912 \mathrm{E}+05$ \\
\hline & 143623.560 & 184642.760 & $3.738 \mathrm{E}-01$ & 4 & 6 & $2.547 \mathrm{E}-04$ & $8.177 \mathrm{E}-03$ & $1.906 \mathrm{E}+05$ \\
\hline & 143623.560 & 184642.760 & $3.738 \mathrm{E}-01$ & 4 & 4 & $2.830 \mathrm{E}-05$ & $9.086 \mathrm{E}-04$ & $3.176 \mathrm{E}+04$ \\
\hline & 143488.950 & 184642.760 & $3.750 \mathrm{E}-01$ & 2 & 4 & $2.839 \mathrm{E}-04$ & $4.543 \mathrm{E}-03$ & $1.604 E+05$ \\
\hline \multirow[t]{3}{*}{$w^{2} P^{o} \rightarrow d^{2} S^{e}$} & 0.2969 & 0.1797 & $1.173 \mathrm{E}-01$ & 6 & 2 & $4.995 E-04$ & $7.667 \mathrm{E}-02$ & $1.656 \mathrm{E}+05$ \\
\hline & 156276.830 & 169109.540 & $1.169 \mathrm{E}-01$ & 4 & 2 & $4.979 E-04$ & $5.112 \mathrm{E}-02$ & $1.093 E+05$ \\
\hline & 156167.040 & 169109.540 & $1.179 \mathrm{E}-01$ & 2 & 2 & $5.022 \mathrm{E}-04$ & $2.556 \mathrm{E}-02$ & $5.607 \mathrm{E}+04$ \\
\hline \multirow[t]{5}{*}{$w^{2} P^{o} \rightarrow f^{2} P^{e}$} & 0.2969 & 0.2720 & $2.497 \mathrm{E}-02$ & 6 & 6 & $1.587 \mathrm{E}-02$ & $1.144 \mathrm{E}+01$ & $7.945 \mathrm{E}+04$ \\
\hline & 156276.830 & 158828.310 & $2.330 \mathrm{E}-02$ & 4 & 4 & $1.234 \mathrm{E}-02$ & $6.355 E+00$ & $5.381 \mathrm{E}+04$ \\
\hline & 156276.830 & 159283.660 & $2.740 \mathrm{E}-02$ & 4 & 2 & $2.902 E-03$ & $1.271 \mathrm{E}+00$ & $3.500 \mathrm{E}+04$ \\
\hline & 156167.040 & 158828.310 & $2.430 \mathrm{E}-02$ & 2 & 4 & $5.148 E-03$ & $1.271 \mathrm{E}+00$ & $1.221 \mathrm{E}+04$ \\
\hline & 156167.040 & 159283.660 & $2.840 \mathrm{E}-02$ & 2 & 2 & $1.203 \mathrm{E}-02$ & $2.542 \mathrm{E}+00$ & $7.796 \mathrm{E}+04$ \\
\hline \multirow[t]{5}{*}{$w^{2} P^{\circ} \rightarrow g^{2} P^{e}$} & 0.2969 & 0.2064 & $9.052 \mathrm{E}-02$ & 6 & 6 & $1.604 \mathrm{E}-02$ & $3.189 \mathrm{E}+00$ & $1.056 \mathrm{E}+06$ \\
\hline & 156276.830 & 166185.470 & $9.030 \mathrm{E}-02$ & 4 & 4 & $1.333 \mathrm{E}-02$ & $1.772 \mathrm{E}+00$ & $8.733 E+05$ \\
\hline & 156276.830 & 166151.610 & $9.000 \mathrm{E}-02$ & 4 & 2 & $2.658 E-03$ & $3.544 \mathrm{E}-01$ & $3.458 E+05$ \\
\hline & 156167.040 & 166185.470 & $9.130 \mathrm{E}-02$ & 2 & 4 & $5.392 \mathrm{E}-03$ & $3.544 \mathrm{E}-01$ & $1.805 E+05$ \\
\hline & 156167.040 & 166151.610 & $9.100 \mathrm{E}-02$ & 2 & 2 & $1.075 \mathrm{E}-02$ & $7.087 \mathrm{E}-01$ & $7.150 \mathrm{E}+05$ \\
\hline \multirow[t]{5}{*}{$w^{2} P^{o} \rightarrow h^{2} P^{c}$} & 0.2969 & 0.2017 & $9.518 \mathrm{E}-02$ & 6 & 6 & $9.706 \mathrm{E}-04$ & $1.835 \mathrm{E}-01$ & $7.063 E+04$ \\
\hline & 156276.830 & 166862.710 & $9.650 \mathrm{E}-02$ & 4 & 4 & $8.200 \mathrm{E}-04$ & $1.020 \mathrm{E}-01$ & $6.133 \mathrm{E}+04$ \\
\hline & 156276.830 & 166331.190 & $9.160 \mathrm{E}-02$ & 4 & 2 & $1.557 \mathrm{E}-04$ & $2.039 \mathrm{E}-02$ & $2.098 \mathrm{E}+04$ \\
\hline & 156167.040 & 166862.710 & $9.750 \mathrm{E}-02$ & 2 & 4 & $3.314 E-04$ & $2.039 \mathrm{E}-02$ & $1.265 \mathrm{E}+04$ \\
\hline & 156167.040 & 166331.190 & $9.260 \mathrm{E}-02$ & 2 & 2 & $6.295 \mathrm{E}-04$ & $4.079 \mathrm{E}-02$ & $4.335 \mathrm{E}+04$ \\
\hline \multirow[t]{5}{*}{$w^{2} P^{\circ} \rightarrow i^{2} P^{e}$} & 0.2969 & 0.1765 & $1.204 \mathrm{E}-01$ & 6 & 6 & $1.556 \mathrm{E}-02$ & $2.326 \mathrm{E}+00$ & $1.813 E+06$ \\
\hline & 156276.830 & 169340.390 & $1.190 \mathrm{E}-01$ & 4 & 4 & $1.282 \mathrm{E}-02$ & $1.292 \mathrm{E}+00$ & $1.458 \mathrm{E}+06$ \\
\hline & 156276.830 & 169687.520 & $1.222 \mathrm{E}-01$ & 4 & 2 & $2.632 \mathrm{E}-03$ & $2.585 \mathrm{E}-01$ & $6.314 \mathrm{E}+05$ \\
\hline & 156167.040 & 169340.390 & $1.200 \mathrm{E}-01$ & 2 & 4 & $5.169 \mathrm{E}-03$ & $2.585 \mathrm{E}-01$ & $2.990 \mathrm{E}+05$ \\
\hline & 156167.040 & 169687.520 & $1.232 \mathrm{E}-01$ & 2 & 2 & $1.061 \mathrm{E}-02$ & $5.169 \mathrm{E}-01$ & $1.294 \mathrm{E}+06$ \\
\hline \multirow[t]{5}{*}{$w^{2} P^{o} \rightarrow j^{2} P^{e}$} & 0.2969 & 0.1342 & $1.627 \mathrm{E}-01$ & 6 & 6 & $2.521 E-03$ & $2.788 \mathrm{E}-01$ & $5.363 E+05$ \\
\hline & 156276.830 & 174274.520 & $1.640 \mathrm{E}-01$ & 4 & 4 & $2.117 \mathrm{E}-03$ & $1.549 \mathrm{E}-01$ & $4.573 E+05$ \\
\hline & 156276.830 & 173749.010 & $1.592 \mathrm{E}-01$ & 4 & 2 & $4.110 \mathrm{E}-04$ & $3.098 \mathrm{E}-02$ & $1.673 \mathrm{E}+05$ \\
\hline & 156167.040 & 174274.520 & $1.650 \mathrm{E}-01$ & 2 & 4 & $8.519 \mathrm{E}-04$ & $3.098 \mathrm{E}-02$ & $9.314 E+04$ \\
\hline & 156167.040 & 173749.010 & $1.602 \mathrm{E}-01$ & 2 & 2 & $1.654 \mathrm{E}-03$ & $6.196 \mathrm{E}-02$ & $3.410 \mathrm{E}+05$ \\
\hline \multirow[t]{4}{*}{$w^{2} P^{o} \rightarrow f^{2} D^{c}$} & 0.2969 & 0.2673 & $2.965 \mathrm{E}-02$ & 6 & 10 & $5.422 \mathrm{E}-03$ & $3.291 \mathrm{E}+00$ & $2.297 \mathrm{E}+04$ \\
\hline & 156276.830 & 159492.830 & $2.930 \mathrm{E}-02$ & 4 & 6 & $4.822 \mathrm{E}-03$ & $1.975 \mathrm{E}+00$ & $2.217 \mathrm{E}+04$ \\
\hline & 156276.830 & 159495.940 & $2.930 \mathrm{E}-02$ & 4 & 4 & $5.357 \mathrm{E}-04$ & $2.194 \mathrm{E}-01$ & $3.694 \mathrm{E}+03$ \\
\hline & 156167.040 & 159495.940 & $3.030 \mathrm{E}-02$ & 2 & 4 & $5.540 \mathrm{E}-03$ & $1.097 \mathrm{E}+00$ & $2.043 \mathrm{E}+04$ \\
\hline
\end{tabular}


Table IV. Continued

\begin{tabular}{|c|c|c|c|c|c|c|c|c|}
\hline Transition & $\begin{array}{l}E_{l} \\
\mathrm{~cm}^{-1}\end{array}$ & $\begin{array}{l}E_{f} \\
\mathrm{~cm}^{-1}\end{array}$ & $\begin{array}{l}E_{f i} \\
\mathrm{Ry}\end{array}$ & $g_{i}$ & $g_{f}$ & $f_{i f}$ & $S$ & $\begin{array}{l}A_{f i} \\
\mathrm{~s}^{-1} \\
\end{array}$ \\
\hline \multirow[t]{4}{*}{$w^{2} P^{o} \rightarrow g^{2} D^{e}$} & 0.2969 & 0.2469 & $5.007 \mathrm{E}-02$ & 6 & 10 & $5.202 \mathrm{E}-03$ & $1.870 E+00$ & $6.286 \mathrm{E}+04$ \\
\hline & 156276.830 & 161733.100 & $4.970 \mathrm{E}-02$ & 4 & 6 & $4.647 \mathrm{E}-03$ & $1.122 \mathrm{E}+00$ & $6.147 \mathrm{E}+04$ \\
\hline & 156276.830 & 161737.990 & $4.980 \mathrm{E}-02$ & 4 & 4 & $5.174 \mathrm{E}-04$ & $1.247 \mathrm{E}-01$ & $1.031 E+04$ \\
\hline & 156167.040 & 161737.990 & $5.080 \mathrm{E}-02$ & 2 & 4 & $5.278 \mathrm{E}-03$ & $6.234 \mathrm{E}-01$ & $5.470 \mathrm{E}+04$ \\
\hline \multirow{4}{*}{$\mathrm{w}^{2} \mathrm{P}^{\mathrm{o}} \rightarrow \mathrm{h}^{2} \mathrm{D}^{\mathrm{e}}$} & 0.2969 & 0.1944 & $1.025 \mathrm{E}-01$ & 6 & 10 & $2.465 \mathrm{E}-03$ & $4.326 \mathrm{E}-01$ & $1.249 \mathrm{E}+05$ \\
\hline & 156276.830 & 167506.390 & $1.023 \mathrm{E}-01$ & 4 & 6 & $2.213 \mathrm{E}-03$ & $2.596 \mathrm{E}-01$ & $1.240 \mathrm{E}+05$ \\
\hline & 156276.830 & 167472.420 & $1.020 \mathrm{E}-01$ & 4 & 4 & $2.452 \mathrm{E}-04$ & $2.884 \mathrm{E}-02$ & $2.049 \mathrm{E}+04$ \\
\hline & 156167.040 & 167472.420 & $1.030 \mathrm{E}-01$ & 2 & 4 & $2.476 \mathrm{E}-03$ & $1.442 \mathrm{E}-01$ & $1.055 \mathrm{E}+05$ \\
\hline \multirow{4}{*}{$w^{2} P^{\circ} \rightarrow i^{2} D^{e}$} & 0.2969 & 0.1717 & $1.252 \mathrm{E}-01$ & 6 & 10 & $9.950 \mathrm{E}-02$ & $1.431 \mathrm{E}+01$ & $7.515 \mathrm{E}+06$ \\
\hline & 156276.830 & 170015.230 & $1.252 \mathrm{E}-01$ & 4 & 6 & $8.955 \mathrm{E}-02$ & $8.584 E+00$ & $7.517 \mathrm{E}+06$ \\
\hline & 156276.830 & 169922.270 & $1.243 \mathrm{E}-01$ & 4 & 4 & $9.879 \mathrm{E}-03$ & $9.537 \mathrm{E}-01$ & $1.226 \mathrm{E}+06$ \\
\hline & 156167.040 & 169922.270 & $1.253 \mathrm{E}-01$ & 2 & 4 & $9.958 \mathrm{E}-02$ & $4.769 \mathrm{E}+00$ & $6.279 \mathrm{E}+06$ \\
\hline \multirow{4}{*}{$w^{2} P^{o} \rightarrow j^{2} D^{e}$} & 0.2969 & 0.1071 & $1.899 \mathrm{E}-01$ & 6 & 10 & $1.475 \mathrm{E}-05$ & $1.398 \mathrm{E}-03$ & $2.563 \mathrm{E}+03$ \\
\hline & 156276.830 & 177076.280 & $1.895 \mathrm{E}-01$ & 4 & 6 & $1.325 \mathrm{E}-05$ & $8.390 E-04$ & $2.548 \mathrm{E}+03$ \\
\hline & 156276.830 & 177077.020 & $1.895 \mathrm{E}-01$ & 4 & 4 & $1.472 \mathrm{E}-06$ & $9.323 \mathrm{E}-05$ & $4.246 \mathrm{E}+02$ \\
\hline & 156167.040 & 177077.020 & $1.905 \mathrm{E}-01$ & 2 & 4 & $1.480 \mathrm{E}-05$ & $4.661 \mathrm{E}-04$ & $2.157 \mathrm{E}+03$ \\
\hline \multirow[t]{4}{*}{$w^{2} P^{0} \rightarrow k^{2} D^{0}$} & 0.2969 & 0.0381 & $2.588 \mathrm{E}-01$ & 6 & 10 & $5.137 \mathrm{E}-03$ & $3.573 E-01$ & $1.658 \mathrm{E}+06$ \\
\hline & 156276.830 & 184642.760 & $2.585 \mathrm{E}-01$ & 4 & 6 & $4.618 \mathrm{E}-03$ & $2.144 \mathrm{E}-01$ & $1.652 \mathrm{E}+06$ \\
\hline & 156276.830 & 184642.760 & $2.585 \mathrm{E}-01$ & 4 & 4 & $5.131 \mathrm{E}-04$ & $2.382 \mathrm{E}-02$ & $2.754 \mathrm{E}+05$ \\
\hline & 156167.040 & 184642.760 & $2.595 \mathrm{E}-01$ & 2 & 4 & $5.150 \mathrm{E}-03$ & $1.191 \mathrm{E}-01$ & $1.393 \mathrm{E}+06$ \\
\hline \multirow[t]{4}{*}{$\mathrm{z}^{2} \mathrm{D}^{\circ} \rightarrow \mathrm{a}^{2} \mathrm{P}^{\mathrm{e}}$} & 1.5852 & 0.7571 & $8.281 E-01$ & 10 & 6 & $4.402 \mathrm{E}-03$ & $1.595 \mathrm{E}-01$ & $4.042 \mathrm{E}+07$ \\
\hline & 14884.730 & 105599.060 & $8.267 \mathrm{E}-01$ & 6 & 4 & $4.394 \mathrm{E}-03$ & $9.569 \mathrm{E}-02$ & $3.618 \mathrm{E}+07$ \\
\hline & 14852.940 & 105599.060 & $8.269 \mathrm{E}-01$ & 4 & 4 & $7.327 \mathrm{E}-04$ & $1.063 \mathrm{E}-02$ & $4.024 \mathrm{E}+06$ \\
\hline & 14852.940 & 106044.240 & $8.310 \mathrm{E}-01$ & 4 & 2 & $3.681 \mathrm{E}-03$ & $5.316 \mathrm{E}-02$ & $4.084 \mathrm{E}+07$ \\
\hline \multirow[t]{4}{*}{$\mathrm{z}^{2} \mathrm{D}^{\circ} \rightarrow \mathrm{b}^{2} \mathrm{P}^{\mathrm{e}}$} & 1.5852 & 0.6884 & $8.968 \mathrm{E}-01$ & 10 & 6 & $1.451 \mathrm{E}-01$ & $4.853 \mathrm{E}+00$ & $1.562 \mathrm{E}+09$ \\
\hline & 14884.730 & 113461.540 & $8.983 \mathrm{E}-01$ & 6 & 4 & $1.453 \mathrm{E}-01$ & $2.912 \mathrm{E}+00$ & $1.413 \mathrm{E}+09$ \\
\hline & 14852.940 & 113461.540 & $8.986 \mathrm{E}-01$ & 4 & 4 & $2.423 \mathrm{E}-02$ & $3.235 \mathrm{E}-01$ & $1.571 \mathrm{E}+08$ \\
\hline & 14852.940 & 112937.570 & $8.939 \mathrm{E}-01$ & 4 & 2 & $1.205 \mathrm{E}-01$ & $1.618 \mathrm{E}+00$ & $1.547 \mathrm{E}+09$ \\
\hline \multirow[t]{4}{*}{$z^{2} D^{\circ} \rightarrow c^{2} P^{e}$} & 1.5852 & 0.4453 & $1.140 \mathrm{E}+00$ & 10 & 6 & $7.654 \mathrm{E}-02$ & $2.014 \mathrm{E}+00$ & $1.331 \mathrm{E}+09$ \\
\hline & 14884.730 & 140016.770 & $1.140 \mathrm{E}+00$ & 6 & 4 & $7.656 \mathrm{E}-02$ & $1.209 \mathrm{E}+\infty 0$ & $1.199 \mathrm{E}+09$ \\
\hline & 14852.940 & 140016.770 & $1.141 \mathrm{E}+00$ & 4 & 4 & $1.276 \mathrm{E}-02$ & $1.343 \mathrm{E}-01$ & $1.334 \mathrm{E}+08$ \\
\hline & 14852.940 & 139844.990 & $1.139 \mathrm{E}+00$ & 4 & 2 & $6.373 \mathrm{E}-02$ & $6.714 \mathrm{E}-01$ & $1.328 \mathrm{E}+09$ \\
\hline \multirow[t]{4}{*}{$z^{2} D^{o} \rightarrow d^{2} P^{e}$} & 1.5852 & 0.3936 & $1.192 E+\infty 0$ & 10 & 6 & $2.228 \mathrm{E}-01$ & $5.609 \mathrm{E}+00$ & $4.234 \mathrm{E}+09$ \\
\hline & 14884.730 & 145505.740 & $1.190 \mathrm{E}+00$ & 6 & 4 & $2.225 \mathrm{E}-01$ & $3.365 \mathrm{E}+00$ & $3.798 \mathrm{E}+09$ \\
\hline & 14852.940 & 145505.740 & $1.191 \mathrm{E}+00$ & 4 & 4 & $3.710 \mathrm{E}-02$ & $3.739 \mathrm{E}-01$ & $4.223 \mathrm{E}+08$ \\
\hline & 14852.940 & 145877.660 & $1.194 E+00$ & 4 & 2 & $1.860 \mathrm{E}-01$ & $1.870 \mathrm{E}+00$ & $4.260 \mathrm{E}+09$ \\
\hline \multirow[t]{4}{*}{$\mathrm{z}^{2} \mathrm{D}^{\circ} \rightarrow \mathrm{e}^{2} \mathrm{P}^{\mathrm{e}}$} & 1.5852 & 0.3380 & $1.247 \mathrm{E}+00$ & 10 & 6 & $1.892 \mathrm{E}-02$ & $4.551 \mathrm{E}-01$ & $3.940 \mathrm{E}+08$ \\
\hline & 14884.730 & 151910.830 & $1.249 \mathrm{E}+00$ & 6 & 4 & $1.894 \mathrm{E}-02$ & $2.730 \mathrm{E}-01$ & $3.558 \mathrm{E}+08$ \\
\hline & 14852.940 & 151910.830 & $1.249 \mathrm{E}+00$ & 4 & 4 & $3.158 \mathrm{E}-03$ & $3.034 \mathrm{E}-02$ & $3.956 \mathrm{E}+07$ \\
\hline & 14852.940 & 151383.810 & $1.244 E+00$ & 4 & 2 & $1.573 \mathrm{E}-02$ & $1.517 \mathrm{E}-01$ & $3.911 \mathrm{E}+08$ \\
\hline \multirow[t]{4}{*}{$\mathrm{z}^{2} \mathrm{D}^{\circ} \rightarrow \mathrm{f}^{2} \mathrm{P}^{\circ}$} & 1.5852 & 0.2720 & $1.313 \mathrm{E}+00$ & 10 & 6 & $1.277 \mathrm{E}-01$ & $2.918 \mathrm{E}+00$ & $2.948 \mathrm{E}+09$ \\
\hline & 14884.730 & 158828.310 & $1.312 \mathrm{E}+00$ & 6 & 4 & $1.276 \mathrm{E}-01$ & $1.751 \mathrm{E}+00$ & $2.645 \mathrm{E}+09$ \\
\hline & 14852.940 & 158828.310 & $1.312 \mathrm{E}+00$ & 4 & 4 & $2.127 \mathrm{E}-02$ & $1.945 \mathrm{E}-01$ & $2.941 \mathrm{E}+08$ \\
\hline & 14852.940 & 159283.660 & $1.316 \mathrm{E}+00$ & 4 & 2 & $1.067 \mathrm{E}-01$ & $9.725 \mathrm{E}-01$ & $2.968 \mathrm{E}+09$ \\
\hline \multirow[t]{4}{*}{$\mathrm{z}^{2} \mathrm{D}^{\circ} \rightarrow \mathrm{g}^{2} \mathrm{P}^{\mathrm{e}}$} & 1.5852 & 0.2064 & $1.379 \mathrm{E}+00$ & 10 & 6 & $4.077 \mathrm{E}-02$ & $8.872 E-01$ & $1.038 \mathrm{E}+09$ \\
\hline & 14884.730 & 166185.470 & $1.379 \mathrm{E}+00$ & 6 & 4 & $4.077 \mathrm{E}-02$ & $5.323 \mathrm{E}-01$ & $9.339 \mathrm{E}+08$ \\
\hline & 14852.940 & 166185.470 & $1.379 \mathrm{E}+00$ & 4 & 4 & $6.797 \mathrm{E}-03$ & $5.914 \mathrm{E}-02$ & $1.038 \mathrm{E}+08$ \\
\hline & 14852.940 & 166151.610 & $1.379 \mathrm{E}+00$ & 4 & 2 & $3.398 \mathrm{E}-02$ & $2.957 \mathrm{E}-01$ & $1.038 \mathrm{E}+09$ \\
\hline
\end{tabular}


Table IV. Continued

\begin{tabular}{|c|c|c|c|c|c|c|c|c|}
\hline Transition & $\begin{array}{l}E_{i} \\
\mathrm{~cm}^{-1}\end{array}$ & $\begin{array}{l}E_{f} \\
\mathrm{~cm}^{-1} \\
\end{array}$ & $\begin{array}{l}E_{f i} \\
\mathrm{Ry} \\
\end{array}$ & $g_{i}$ & $g_{f}$ & $f_{i f}$ & $s$ & $\begin{array}{l}A_{f i} \\
\mathrm{~s}^{-1} \\
\end{array}$ \\
\hline \multirow[t]{4}{*}{$\mathrm{z}^{2} \mathrm{D}^{\circ} \rightarrow \mathrm{h}^{2} \mathrm{P}^{\mathrm{e}}$} & 1.5852 & 0.2017 & $1.383 \mathrm{E}+00$ & 10 & 6 & $9.519 \mathrm{E}-03$ & $2.064 \mathrm{E}-01$ & $2.439 E+08$ \\
\hline & 14884.730 & 166862.710 & $1.385 \mathrm{E}+00$ & 6 & 4 & $9.530 \mathrm{E}-03$ & $1.239 \mathrm{E}-01$ & $2.202 \mathrm{E}+08$ \\
\hline & 14852.940 & 166862.710 & $1.385 \mathrm{E}+00$ & 4 & 4 & $1.589 \mathrm{E}-03$ & $1.376 \mathrm{E}-02$ & $2.449 E+07$ \\
\hline & 14852.940 & 166331.190 & $1.380 \mathrm{E}+00$ & 4 & 2 & $7.915 \mathrm{E}-03$ & $6.881 \mathrm{E}-02$ & $2.423 \mathrm{E}+08$ \\
\hline \multirow[t]{4}{*}{$\mathrm{z}^{2} \mathrm{D}^{\circ} \rightarrow \mathrm{i}^{2} \mathrm{P}^{e}$} & 1.5852 & 0.1765 & $1.409 \mathrm{E}+00$ & 10 & 6 & $1.789 \mathrm{E}-02$ & $3.810 \mathrm{E}-01$ & $4.753 E+08$ \\
\hline & 14884.730 & 169340.390 & $1.407 \mathrm{E}+00$ & 6 & 4 & $1.788 \mathrm{E}-02$ & $2.286 \mathrm{E}-01$ & $4.266 \mathrm{E}+08$ \\
\hline & 14852.940 & 169340.390 & $1.408 \mathrm{E}+00$ & 4 & 4 & $2.980 \mathrm{E}-03$ & $2.540 \mathrm{E}-02$ & $4.743 \mathrm{E}+07$ \\
\hline & 14852.940 & 169687.520 & $1.411 \mathrm{E}+00$ & 4 & 2 & $1.493 \mathrm{E}-02$ & $1.270 \mathrm{E}-01$ & $4.776 \mathrm{E}+08$ \\
\hline \multirow[t]{4}{*}{$\mathrm{z}^{2} \mathrm{D}^{\circ} \rightarrow \mathrm{j}^{2} \mathrm{P}^{\mathrm{e}}$} & 1.5852 & 0.1342 & $1.451 \mathrm{E}+00$ & 10 & 6 & $3.134 E-03$ & $6.480 \mathrm{E}-02$ & $8.833 \mathrm{E}+07$ \\
\hline & 14884.730 & 174274.520 & $1.452 \mathrm{E}+00$ & 6 & 4 & $3.137 \mathrm{E}-03$ & $3.888 \mathrm{E}-02$ & $7.974 \mathrm{E}+07$ \\
\hline & 14852.940 & 174274.520 & $1.453 \mathrm{E}+00$ & 4 & 4 & $5.230 \mathrm{E}-04$ & $4.320 \mathrm{E}-03$ & $8.865 \mathrm{E}+06$ \\
\hline & 14852.940 & 173749.010 & $1.448 \mathrm{E}+00$ & 4 & 2 & $2.606 \mathrm{E}-03$ & $2.160 \mathrm{E}-02$ & $8.778 \mathrm{E}+07$ \\
\hline \multirow{5}{*}{$\mathrm{z}^{2} \mathrm{D}^{\circ} \rightarrow \mathrm{a}^{2} \mathrm{D}^{\mathrm{e}}$} & 1.5852 & 0.8285 & $7.567 \mathrm{E}-01$ & 10 & 10 & $1.555 \mathrm{E}-02$ & $6.166 \mathrm{E}-01$ & $7.153 E+07$ \\
\hline & 14884.730 & 97918.860 & $7.567 \mathrm{E}-01$ & 6 & 6 & $1.452 \mathrm{E}-02$ & $3.453 E-01$ & $6.675 E+07$ \\
\hline & 14884.730 & 97890.740 & $7.564 \mathrm{E}-01$ & 6 & 4 & $1.036 \mathrm{E}-03$ & $2.467 \mathrm{E}-02$ & $7.145 E+06$ \\
\hline & 14852.940 & 97918.860 & $7.569 \mathrm{E}-01$ & 4 & 6 & $1.556 \mathrm{E}-03$ & $2.467 \mathrm{E}-02$ & $4.774 \mathrm{E}+06$ \\
\hline & 14852.940 & 97890.740 & $7.567 \mathrm{E}-01$ & 4 & 4 & $1.400 \mathrm{E}-02$ & $2.220 \mathrm{E}-01$ & $6.438 \mathrm{E}+07$ \\
\hline \multirow{5}{*}{$z^{2} D^{\circ} \rightarrow b^{2} D^{e}$} & 1.5852 & 0.6132 & $9.719 \mathrm{E}-01$ & 10 & 10 & $1.457 \mathrm{E}-01$ & $4.497 \mathrm{E}+\infty 0$ & $1.105 \mathrm{E}+09$ \\
\hline & 14884.730 & 121530.020 & $9.719 E-01$ & 6 & 6 & $1.360 \mathrm{E}-01$ & $2.518 \mathrm{E}+00$ & $1.032 E+09$ \\
\hline & 14884.730 & 121528.720 & $9.719 \mathrm{E}-01$ & 6 & 4 & $9.712 \mathrm{E}-03$ & $1.799 \mathrm{E}-01$ & $1.105 \mathrm{E}+08$ \\
\hline & 14852.940 & 121530.020 & $9.722 \mathrm{E}-01$ & 4 & 6 & $1.457 \mathrm{E}-02$ & $1.799 \mathrm{E}-01$ & $7.375 E+07$ \\
\hline & 14852.940 & 121528.720 & $9.722 \mathrm{E}-01$ & 4 & 4 & $1.312 \mathrm{E}-01$ & $1.619 \mathrm{E}+00$ & $9.956 \mathrm{E}+08$ \\
\hline \multirow{5}{*}{$\mathrm{z}^{2} \mathrm{D}^{\circ} \rightarrow \mathrm{c}^{2} \mathrm{D}^{\mathrm{e}}$} & 1.5852 & 0.5029 & $1.082 E+00$ & 10 & 10 & $1.369 \mathrm{E}-01$ & $3.794 \mathrm{E}+00$ & $1.288 \mathrm{E}+09$ \\
\hline & 14884.730 & 133814.840 & $1.084 E+\infty 00$ & 6 & 6 & $1.279 \mathrm{E}-01$ & $2.125 \mathrm{E}+00$ & $1.207 \mathrm{E}+09$ \\
\hline & 14884.730 & 133360.860 & $1.080 \mathrm{E}+\infty 0$ & 6 & 4 & $9.103 E-03$ & $1.518 \mathrm{E}-01$ & $1.278 \mathrm{E}+08$ \\
\hline & 14852.940 & 133814.840 & $1.084 \mathrm{E}+00$ & 4 & 6 & $1.371 \mathrm{E}-02$ & $1.518 \mathrm{E}-01$ & $8.627 \mathrm{E}+07$ \\
\hline & 14852.940 & 133360.860 & $1.080 \mathrm{E}+\infty 0$ & 4 & 4 & $1.229 \mathrm{E}-01$ & $1.366 \mathrm{E}+00$ & $1.152 \mathrm{E}+09$ \\
\hline \multirow[t]{5}{*}{$z^{2} D^{\circ} \rightarrow d^{2} D^{e}$} & 1.5852 & 0.4079 & $1.177 \mathrm{E}+\infty$ & 10 & 10 & $4.415 \mathrm{E}-01$ & $1.125 \mathrm{E}+01$ & $4.915 \mathrm{E}+09$ \\
\hline & 14884.730 & 144009.420 & $1.177 \mathrm{E}+00$ & 6 & 6 & $4.118 \mathrm{E}-01$ & $6.300 \mathrm{E}+00$ & $4.580 \mathrm{E}+09$ \\
\hline & 14884.730 & 144142.160 & $1.178 \mathrm{E}+00$ & 6 & 4 & $2.945 \mathrm{E}-02$ & $4.500 \mathrm{E}-01$ & $4.922 \mathrm{E}+08$ \\
\hline & 14852.940 & 144009.420 & $1.177 \mathrm{E}+00$ & 4 & 6 & $4.413 \mathrm{E}-02$ & $4.500 \mathrm{E}-01$ & $3.274 \mathrm{E}+08$ \\
\hline & 14852.940 & 144142.160 & $1.178 \mathrm{E}+00$ & 4 & 4 & $3.976 \mathrm{E}-01$ & $4.050 \mathrm{E}+00$ & $4.433 \mathrm{E}+09$ \\
\hline \multirow[t]{5}{*}{$z^{2} D^{\circ} \rightarrow e^{2} D^{e}$} & 1.5852 & 0.3639 & $1.221 \mathrm{E}+\infty 0$ & 10 & 10 & $1.011 \mathrm{E}-01$ & $2.484 \mathrm{E}+00$ & $1.211 \mathrm{E}+09$ \\
\hline & 14884.730 & 148886.570 & $1.221 \mathrm{E}+00$ & 6 & 6 & $9.436 \mathrm{E}-02$ & $1.391 \mathrm{E}+\infty 0$ & $1.130 \mathrm{E}+09$ \\
\hline & 14884.730 & 148900.910 & $1.221 \mathrm{E}+00$ & 6 & 4 & $6.741 \mathrm{E}-03$ & $9.935 \mathrm{E}-02$ & $1.211 \mathrm{E}+08$ \\
\hline & 14852.940 & 148886.570 & $1.221 \mathrm{E}+00$ & 4 & 6 & $1.011 \mathrm{E}-02$ & $9.935 \mathrm{E}-02$ & $8.079 \mathrm{E}+07$ \\
\hline & 14852.940 & 148900.910 & $1.222 \mathrm{E}+00$ & 4 & 4 & $9.102 \mathrm{E}-02$ & $8.942 \mathrm{E}-01$ & $1.091 \mathrm{E}+09$ \\
\hline \multirow[t]{5}{*}{$\mathrm{z}^{2} \mathrm{D}^{\circ} \rightarrow \mathrm{f}^{2} \mathrm{D}^{\mathrm{e}}$} & 1.5852 & 0.2673 & $1.318 \mathrm{E}+\infty 0$ & 10 & 10 & $2.069 \mathrm{E}-02$ & $4.709 \mathrm{E}-01$ & $2.886 \mathrm{E}+08$ \\
\hline & 14884.730 & 159492.830 & $1.318 \mathrm{E}+\infty 0$ & 6 & 6 & $1.930 \mathrm{E}-02$ & $2.637 \mathrm{E}-01$ & $2.693 E+08$ \\
\hline & 14884.730 & 159495.940 & $1.318 \mathrm{E}+\infty$ & 6 & 4 & $1.379 \mathrm{E}-03$ & $1.883 E-02$ & $2.885 \mathrm{E}+07$ \\
\hline & 14852.940 & 159492.830 & $1.318 \mathrm{E}+\infty 0$ & 4 & 6 & $2.069 \mathrm{E}-03$ & $1.883 \mathrm{E}-02$ & $1.925 \mathrm{E}+07$ \\
\hline & 14852.940 & 159495.940 & $1.318 \mathrm{E}+\infty 0$ & 4 & 4 & $1.862 \mathrm{E}-02$ & $1.695 \mathrm{E}-01$ & $2.598 \mathrm{E}+08$ \\
\hline \multirow[t]{5}{*}{$z^{2} D^{\circ} \rightarrow g^{2} D^{e}$} & 1.5852 & 0.2469 & $1.338 \mathrm{E}+\infty 0$ & 10 & 10 & $2.763 \mathrm{E}-02$ & $6.193 \mathrm{E}-01$ & $3.975 \mathrm{E}+08$ \\
\hline & 14884.730 & 161733.100 & $1.338 \mathrm{E}+00$ & 6 & 6 & $2.578 \mathrm{E}-02$ & $3.468 \mathrm{E}-01$ & $3.708 \mathrm{E}+08$ \\
\hline & 14884.730 & 161737.990 & $1.338 E+\infty$ & 6 & 4 & $1.842 \mathrm{E}-03$ & $2.477 E-02$ & $3.974 E+07$ \\
\hline & 14852.940 & 161733.100 & $1.338 \mathrm{E}+00$ & 4 & 6 & $2.763 \mathrm{E}-03$ & $2.477 \mathrm{E}-02$ & $2.651 \mathrm{E}+07$ \\
\hline & 14852.940 & 161737.990 & $1.339 \mathrm{E}+00$ & 4 & 4 & $2.487 \mathrm{E}-02$ & $2.230 \mathrm{E}-01$ & $3.579 E+08$ \\
\hline \multirow[t]{5}{*}{$\mathrm{z}^{2} \mathrm{D}^{\mathrm{o}} \rightarrow \mathrm{h}^{2} \mathrm{D}^{\mathrm{e}}$} & 1.5852 & 0.1944 & $1.391 \mathrm{E}+\infty 0$ & 10 & 10 & $1.727 \mathrm{E}-01$ & $3.726 \mathrm{E}+00$ & $2.684 \mathrm{E}+09$ \\
\hline & 14884.730 & 167506.390 & $1.391 \mathrm{E}+00$ & 6 & 6 & $1.612 \mathrm{E}-01$ & $2.086 \mathrm{E}+00$ & $2.505 \mathrm{E}+09$ \\
\hline & 14884.730 & 167472.420 & $1.390 E+\infty 0$ & 6 & 4 & $1.151 \mathrm{E}-02$ & $1.490 \mathrm{E}-01$ & $2.682 \mathrm{E}+08$ \\
\hline & 14852.940 & 167506.390 & $1.391 \mathrm{E}+\infty 0$ & 4 & 6 & $1.728 \mathrm{E}-02$ & $1.490 \mathrm{E}-01$ & $1.790 \mathrm{E}+08$ \\
\hline & 14852.940 & 167472.420 & $1.391 \mathrm{E}+\infty 0$ & 4 & 4 & $1.554 \mathrm{E}-01$ & $1.341 \mathrm{E}+00$ & $2.415 \mathrm{E}+09$ \\
\hline
\end{tabular}


Table IV. Continued

\begin{tabular}{|c|c|c|c|c|c|c|c|c|}
\hline Transition & $\begin{array}{l}E_{i} \\
\mathrm{~cm}^{-1}\end{array}$ & $\begin{array}{l}E_{f} \\
\mathrm{~cm}^{-1}\end{array}$ & $\begin{array}{l}E_{f i} \\
\mathrm{Ry}\end{array}$ & $g_{i}$ & $g_{f}$ & $f_{i f}$ & $S$ & $\begin{array}{l}A_{f i} \\
\mathrm{~s}^{-1} \\
\end{array}$ \\
\hline \multirow{5}{*}{$\mathrm{z}^{2} \mathrm{D}^{\circ} \rightarrow \mathrm{i}^{2} \mathrm{D}^{*}$} & 1.5852 & 0.1717 & $1.413 \mathrm{E}+00$ & 10 & 10 & $5.552 \mathrm{E}-05$ & $1.179 \mathrm{E}-03$ & $8.910 \mathrm{E}+05$ \\
\hline & 14884.730 & 170015.230 & $1.414 \mathrm{E}+\infty$ & 6 & 6 & $5.183 \mathrm{E}-05$ & $6.600 \mathrm{E}-04$ & $8.320 E+05$ \\
\hline & 14884.730 & 169922.270 & $1.413 E+\infty$ & 6 & 4 & $3.700 \mathrm{E}-06$ & $4.714 \mathrm{E}-05$ & $8.897 \mathrm{E}+04$ \\
\hline & 14852.940 & 170015.230 & $1.414 \mathrm{E}+\infty$ & 4 & 6 & $5.555 \mathrm{E}-06$ & $4.714 \mathrm{E}-05$ & $5.946 \mathrm{E}+04$ \\
\hline & 14852.940 & 169922.270 & $1.413 \mathrm{E}+\infty 0$ & 4 & 4 & $4.996 \mathrm{E}-05$ & $4.243 E-04$ & $8.012 \mathrm{E}+05$ \\
\hline \multirow{5}{*}{$z^{2} D^{\circ} \rightarrow j^{2} D^{\bullet}$} & 1.5852 & 0.1071 & $1.478 \mathrm{E}+\infty$ & 10 & 10 & $8.933 \mathrm{E}-03$ & $1.813 \mathrm{E}-01$ & $1.568 \mathrm{E}+08$ \\
\hline & 14884.730 & 177076.280 & $1.478 \mathrm{E}+00$ & 6 & 6 & $8.336 \mathrm{E}-03$ & $1.015 \mathrm{E}-01$ & $1.463 \mathrm{E}+08$ \\
\hline & 14884.730 & 177077.020 & $1.478 \mathrm{E}+00$ & 6 & 4 & $5.955 \mathrm{E}-04$ & $7.252 \mathrm{E}-03$ & $1.567 \mathrm{E}+07$ \\
\hline & 14852.940 & 177076.280 & $1.478 \mathrm{E}+00$ & 4 & 6 & $8.934 \mathrm{E}-04$ & $7.252 \mathrm{E}-03$ & $1.045 \mathrm{E}+07$ \\
\hline & 14852.940 & 177077.020 & $1.478 \mathrm{E}+\infty 0$ & 4 & 4 & $8.040 \mathrm{E}-03$ & $6.527 \mathrm{E}-02$ & $1.411 \mathrm{E}+08$ \\
\hline \multirow[t]{5}{*}{$\mathrm{z}^{2} \mathrm{D}^{\circ} \rightarrow \mathrm{k}^{2} \mathrm{D}^{\mathrm{e}}$} & 1.5852 & 0.0381 & $1.547 \mathrm{E}+00$ & 10 & 10 & $9.396 \mathrm{E}-04$ & $1.822 \mathrm{E}-02$ & $1.806 \mathrm{E}+07$ \\
\hline & 14884.730 & 184642.760 & $1.547 \mathrm{E}+\infty 0$ & 6 & 6 & $8.769 \mathrm{E}-04$ & $1.020 \mathrm{E}-02$ & $1.685 \mathrm{E}+07$ \\
\hline & 14884.730 & 184642.760 & $1.547 \mathrm{E}+\infty$ & 6 & 4 & $6.263 E-05$ & $7.288 \mathrm{E}-04$ & $1.806 \mathrm{E}+06$ \\
\hline & 14852.940 & 184642.760 & $1.547 \mathrm{E}+\infty 0$ & 4 & 6 & $9.397 \mathrm{E}-05$ & $7.288 \mathrm{E}-04$ & $1.205 E+06$ \\
\hline & 14852.940 & 184642.760 & $1.547 \mathrm{E}+00$ & 4 & 4 & $8.457 \mathrm{E}-04$ & $6.559 \mathrm{E}-03$ & $1.626 \mathrm{E}+07$ \\
\hline \multirow[t]{4}{*}{$\mathrm{z}^{2} \mathrm{D}^{\circ} \rightarrow \mathrm{a}^{2} \mathrm{~F}^{\mathrm{c}}$} & 1.5852 & 0.6720 & $9.132 \mathrm{E}-01$ & 10 & 14 & $1.163 \mathrm{E}-02$ & $3.821 \mathrm{E}-01$ & $5.564 \mathrm{E}+07$ \\
\hline & 14884.730 & 115285.610 & $9.150 \mathrm{E}-01$ & 6 & 8 & $1.110 \mathrm{E}-02$ & $2.183 E-01$ & $5.597 \mathrm{E}+07$ \\
\hline & 14884.730 & 114804.370 & $9.106 \mathrm{E}-01$ & 6 & 6 & $5.522 \mathrm{E}-04$ & $1.092 \mathrm{E}-02$ & $3.678 \mathrm{E}+06$ \\
\hline & 14852.940 & 114804.370 & $9.109 \mathrm{E}-01$ & 4 & 6 & $1.160 \mathrm{E}-02$ & $1.528 \mathrm{E}-01$ & $5.153 \mathrm{E}+07$ \\
\hline \multirow[t]{4}{*}{$z^{2} D^{\circ} \rightarrow b^{2} F^{e}$} & 1.5852 & 0.4584 & $1.127 \mathrm{E}+00$ & 10 & 14 & $9.765 \mathrm{E}-01$ & $2.600 \mathrm{E}+01$ & $7.113 E+09$ \\
\hline & 14884.730 & 138527.980 & $1.127 \mathrm{E}+00$ & 6 & 8 & $9.300 \mathrm{E}-01$ & $1.486 \mathrm{E}+01$ & $7.113 E+09$ \\
\hline & 14884.730 & 138509.170 & $1.127 \mathrm{E}+00$ & 6 & 6 & $4.649 \mathrm{E}-02$ & $7.428 \mathrm{E}-01$ & $4.739 E+08$ \\
\hline & 14852.940 & 138509.170 & $1.127 \mathrm{E}+00$ & 4 & 6 & $9.766 \mathrm{E}-01$ & $1.040 \mathrm{E}+01$ & $6.640 \mathrm{E}+09$ \\
\hline \multirow[t]{4}{*}{$\mathrm{z}^{2} \mathrm{D}^{\circ} \rightarrow \mathrm{c}^{2} \mathrm{~F}^{\mathrm{e}}$} & 1.5852 & 0.2955 & $1.290 \mathrm{E}+00$ & 10 & 14 & $1.956 \mathrm{E}-01$ & $4.550 \mathrm{E}+\infty 0$ & $1.867 \mathrm{E}+09$ \\
\hline & 14884.730 & 156604.170 & $1.291 \mathrm{E}+\infty 0$ & 6 & 8 & $1.865 \mathrm{E}-01$ & $2.600 \mathrm{E}+00$ & $1.874 \mathrm{E}+09$ \\
\hline & 14884.730 & 156121.700 & $1.287 \mathrm{E}+\infty 0$ & 6 & 6 & $9.295 \mathrm{E}-03$ & $1.300 \mathrm{E}-01$ & $1.237 \mathrm{E}+08$ \\
\hline & 14852.940 & 156121.700 & $1.287 \mathrm{E}+00$ & 4 & 6 & $1.952 \mathrm{E}-01$ & $1.820 \mathrm{E}+00$ & $1.733 \mathrm{E}+09$ \\
\hline \multirow{4}{*}{$\mathrm{z}^{2} \mathrm{D}^{\circ} \rightarrow \mathrm{d}^{2} \mathrm{~F}^{\mathrm{e}}$} & 1.5852 & 0.2236 & $1.362 \mathrm{E}+00$ & 10 & 14 & $8.613 \mathrm{E}-02$ & $1.898 \mathrm{E}+00$ & $9.162 \mathrm{E}+08$ \\
\hline & 14884.730 & 164337.610 & $1.362 \mathrm{E}+00$ & 6 & 8 & $8.205 \mathrm{E}-02$ & $1.084 \mathrm{E}+\infty 0$ & $9.169 \mathrm{E}+08$ \\
\hline & 14884.730 & 164232.360 & $1.361 \mathrm{E}+00$ & 6 & 6 & $4.099 \mathrm{E}-03$ & $5.422 \mathrm{E}-02$ & $6.099 E+07$ \\
\hline & 14852.940 & 164232.360 & $1.361 \mathrm{E}+00$ & 4 & 6 & $8.611 \mathrm{E}-02$ & $7.591 \mathrm{E}-01$ & $8.544 \mathrm{E}+08$ \\
\hline \multirow[t]{4}{*}{$z^{2} D^{\circ} \rightarrow e^{2} F^{e}$} & 1.5852 & 0.1759 & $1.409 \mathrm{E}+00$ & 10 & 14 & $1.529 \mathrm{E}-01$ & $3.255 \mathrm{E}+\infty$ & $1.742 E+09$ \\
\hline & 14884.730 & 169702.320 & $1.411 \mathrm{E}+00$ & 6 & 8 & $1.458 \mathrm{E}-01$ & $1.860 \mathrm{E}+\infty$ & $1.748 \mathrm{E}+09$ \\
\hline & 14884.730 & 169283.540 & $1.407 \mathrm{E}+00$ & 6 & 6 & $7.270 \mathrm{E}-03$ & $9.301 \mathrm{E}-02$ & $1.156 \mathrm{E}+08$ \\
\hline & 14852.940 & 169283.540 & $1.407 \mathrm{E}+00$ & 4 & 6 & $1.527 \mathrm{E}-01$ & $1.302 \mathrm{E}+00$ & $1.619 \mathrm{E}+09$ \\
\hline \multirow[t]{4}{*}{$\mathrm{y}^{2} \mathrm{D}^{\circ} \rightarrow \mathrm{c}^{2} \mathrm{P}^{\mathrm{e}}$} & 0.5272 & 0.4453 & $8.193 \mathrm{E}-02$ & 10 & 6 & $5.091 \mathrm{E}-04$ & $1.864 \mathrm{E}-01$ & $4.575 \mathrm{E}+04$ \\
\hline & 131187.190 & 140016.770 & $8.040 \mathrm{E}-02$ & 6 & 4 & $4.996 \mathrm{E}-04$ & $1.119 \mathrm{E}-01$ & $3.891 \mathrm{E}+04$ \\
\hline & 130641.110 & 140016.770 & $8.540 \mathrm{E}-02$ & 4 & 4 & $8.845 \mathrm{E}-05$ & $1.243 \mathrm{E}-02$ & $5.181 \mathrm{E}+03$ \\
\hline & 130641.110 & 139844.990 & $8.390 \mathrm{E}-02$ & 4 & 2 & $4.345 \mathrm{E}-04$ & $6.214 \mathrm{E}-02$ & $4.913 \mathrm{E}+04$ \\
\hline \multirow[t]{4}{*}{$\mathrm{y}^{2} \mathrm{D}^{\circ} \rightarrow \mathrm{d}^{2} \mathrm{P}^{e}$} & 0.5272 & 0.3936 & $1.336 \mathrm{E}-01$ & 10 & 6 & $4.535 \mathrm{E}-04$ & $1.018 \mathrm{E}-01$ & $1.084 \mathrm{E}+05$ \\
\hline & 131187.190 & 145505.740 & $1.304 \mathrm{E}-01$ & 6 & 4 & $4.426 \mathrm{E}-04$ & $6.110 \mathrm{E}-02$ & $9.068 \mathrm{E}+04$ \\
\hline & 130641.110 & 145505.740 & $1.354 \mathrm{E}-01$ & 4 & 4 & $7.660 \mathrm{E}-05$ & $6.789 \mathrm{E}-03$ & $1.128 \mathrm{E}+04$ \\
\hline & 130641.110 & 145877.660 & $1.388 \mathrm{E}-01$ & 4 & 2 & $3.926 \mathrm{E}-04$ & $3.394 \mathrm{E}-02$ & $1.215 \mathrm{E}+05$ \\
\hline \multirow[t]{4}{*}{$y^{2} D^{\circ} \rightarrow e^{2} P^{e}$} & 0.5272 & 0.3380 & $1.892 \mathrm{E}-01$ & 10 & 6 & $2.160 \mathrm{E}-01$ & $3.424 \mathrm{E}+01$ & $1.035 \mathrm{E}+08$ \\
\hline & 131187.190 & 151910.830 & $1.888 \mathrm{E}-01$ & 6 & 4 & $2.155 \mathrm{E}-01$ & $2.054 \mathrm{E}+01$ & $9.254 \mathrm{E}+07$ \\
\hline & 130641.110 & 151910.830 & $1.938 \mathrm{E}-01$ & 4 & 4 & $3.686 \mathrm{E}-02$ & $2.283 \mathrm{E}+00$ & $1.112 \mathrm{E}+07$ \\
\hline & 130641.110 & 151383.810 & $1.890 \mathrm{E}-01$ & 4 & 2 & $1.798 \mathrm{E}-01$ & $1.141 \mathrm{E}+01$ & $1.032 \mathrm{E}+08$ \\
\hline \multirow[t]{4}{*}{$\mathrm{y}^{2} \mathrm{D}^{\circ} \rightarrow \mathrm{f}^{2} \mathrm{P}^{\mathrm{e}}$} & 0.5272 & 0.2720 & $2.553 E-01$ & 10 & 6 & $5.010 \mathrm{E}-04$ & $5.888 \mathrm{E}-02$ & $4.370 \mathrm{E}+05$ \\
\hline & 131187.190 & 158828.310 & $2.519 \mathrm{E}-01$ & 6 & 4 & $4.944 \mathrm{E}-04$ & $3.533 \mathrm{E}-02$ & $3.780 \mathrm{E}+05$ \\
\hline & 130641.110 & 158828.310 & $2.569 \mathrm{E}-01$ & 4 & 4 & $8.404 \mathrm{E}-05$ & $3.926 \mathrm{E}-03$ & $4.455 \mathrm{E}+04$ \\
\hline & 130641.110 & 159283.660 & $2.610 \mathrm{E}-01$ & 4 & 2 & $4.269 \mathrm{E}-04$ & $1.963 \mathrm{E}-02$ & $4.672 \mathrm{E}+05$ \\
\hline
\end{tabular}


Table IV. Continued

\begin{tabular}{|c|c|c|c|c|c|c|c|c|}
\hline Transition & $\begin{array}{l}E_{i} \\
\mathrm{~cm}^{-1}\end{array}$ & $\begin{array}{l}E_{f} \\
\mathrm{~cm}^{-1}\end{array}$ & $\begin{array}{l}E_{f i} \\
\text { Ry }\end{array}$ & $g_{i}$ & $g_{f}$ & $f_{i f}$ & $S$ & $\begin{array}{l}A_{f i} \\
\mathrm{~s}^{-1} \\
\end{array}$ \\
\hline \multirow[t]{4}{*}{$y^{2} D^{\circ} \rightarrow g^{2} P^{e}$} & 0.5272 & 0.2064 & $3.208 \mathrm{E}-01$ & 10 & 6 & $5.045 \mathrm{E}-03$ & $4.717 E-01$ & $6.950 \mathrm{E}+06$ \\
\hline & 131187.190 & 166185.470 & $3.189 \mathrm{E}-01$ & 6 & 4 & $5.014 \mathrm{E}-03$ & $2.830 \mathrm{E}-01$ & $6.144 \mathrm{E}+06$ \\
\hline & 130641.110 & 166185.470 & $3.239 E-01$ & 4 & 4 & $8.488 \mathrm{E}-04$ & $3.145 \mathrm{E}-02$ & $7.153 E+05$ \\
\hline & 130641.110 & 166151.610 & $3.236 \mathrm{E}-01$ & 4 & 2 & $4.240 \mathrm{E}-03$ & $1.572 E-01$ & $7.133 \mathrm{E}+06$ \\
\hline \multirow[t]{4}{*}{$y^{2} D^{o} \rightarrow h^{2} p^{e}$} & 0.5272 & 0.2017 & $3.255 \mathrm{E}-01$ & 10 & 6 & $2.370 \mathrm{E}-02$ & $2.184 \mathrm{E}+00$ & $3.361 \mathrm{E}+07$ \\
\hline & 131187.190 & 166862.710 & $3.251 \mathrm{E}-01$ & 6 & 4 & $2.367 E-02$ & $1.311 \mathrm{E}+00$ & $3.014 E+07$ \\
\hline & 130641.110 & 166862.710 & $3.301 \mathrm{E}-01$ & 4 & 4 & $4.006 \mathrm{E}-03$ & $1.456 \mathrm{E}-01$ & $3.506 \mathrm{E}+06$ \\
\hline & 130641.110 & 166331.190 & $3.252 \mathrm{E}-01$ & 4 & 2 & $1.973 \mathrm{E}-02$ & $7.281 \mathrm{E}-01$ & $3.352 \mathrm{E}+07$ \\
\hline \multirow[t]{4}{*}{$\mathrm{y}^{2} \mathrm{D}^{\circ} \rightarrow \mathrm{i}^{2} \mathrm{P}^{\mathrm{e}}$} & 0.5272 & 0.1765 & $3.507 \mathrm{E}-01$ & 10 & 6 & $9.549 \mathrm{E}-04$ & $8.168 \mathrm{E}-02$ & $1.572 \mathrm{E}+06$ \\
\hline & 131187.190 & 169340.390 & $3.476 \mathrm{E}-01$ & 6 & 4 & $9.464 \mathrm{E}-04$ & $4.901 \mathrm{E}-02$ & $1.378 \mathrm{E}+06$ \\
\hline & 130641.110 & 169340.390 & $3.526 \mathrm{E}-01$ & 4 & 4 & $1.600 \mathrm{E}-04$ & $5.445 \mathrm{E}-03$ & $1.598 \mathrm{E}+05$ \\
\hline & 130641.110 & 169687.520 & $3.558 \mathrm{E}-01$ & 4 & 2 & $8.073 \mathrm{E}-04$ & $2.723 \mathrm{E}-02$ & $1.642 \mathrm{E}+06$ \\
\hline \multirow[t]{4}{*}{$y^{2} D^{o} \rightarrow j^{2} P^{e}$} & 0.5272 & 0.1342 & $3.930 \mathrm{E}-01$ & 10 & 6 & $8.688 \mathrm{E}-03$ & $6.631 \mathrm{E}-01$ & $1.797 \mathrm{E}+07$ \\
\hline & 131187.190 & 174274.520 & $3.926 \mathrm{E}-01$ & 6 & 4 & $8.678 \mathrm{E}-03$ & $3.979 \mathrm{E}-01$ & $1.612 \mathrm{E}+07$ \\
\hline & 130641.110 & 174274.520 & $3.976 \mathrm{E}-01$ & 4 & 4 & $1.465 \mathrm{E}-03$ & $4.421 \mathrm{E}-02$ & $1.860 \mathrm{E}+06$ \\
\hline & 130641.110 & 173749.010 & $3.928 \mathrm{E}-01$ & 4 & 2 & $7.236 \mathrm{E}-03$ & $2.210 \mathrm{E}-01$ & $1.793 \mathrm{E}+07$ \\
\hline \multirow[t]{5}{*}{$y^{2} D^{\circ} \rightarrow c^{2} D^{e}$} & 0.5272 & 0.5029 & $2.428 \mathrm{E}-02$ & 10 & 10 & $8.964 \mathrm{E}-03$ & $1.108 \mathrm{E}+01$ & $4.245 \mathrm{E}+04$ \\
\hline & 131187.190 & 133814.840 & $2.390 \mathrm{E}-02$ & 6 & 6 & $8.235 E-03$ & $6.202 \mathrm{E}+\infty 0$ & $3.778 \mathrm{E}+04$ \\
\hline & 131187.190 & 133360.860 & $1.980 \mathrm{E}-02$ & 6 & 4 & $4.873 \mathrm{E}-04$ & $4.430 \mathrm{E}-01$ & $2.302 \mathrm{E}+03$ \\
\hline & 130641.110 & 133814.840 & $2.890 \mathrm{E}-02$ & 4 & 6 & $1.067 E-03$ & $4.430 \mathrm{E}-01$ & $4.772 \mathrm{E}+03$ \\
\hline & 130641.110 & 133360.860 & $2.480 \mathrm{E}-02$ & 4 & 4 & $8.240 \mathrm{E}-03$ & $3.987 \mathrm{E}+00$ & $4.071 E+04$ \\
\hline \multirow{5}{*}{$y^{2} D^{o} \rightarrow d^{2} D^{e}$} & 0.5272 & 0.4079 & $1.193 \mathrm{E}-01$ & 10 & 10 & $4.673 \mathrm{E}-04$ & $1.175 \mathrm{E}-01$ & $5.344 \mathrm{E}+04$ \\
\hline & 131187.190 & 144009.420 & $1.168 \mathrm{E}-01$ & 6 & 6 & $4.269 \mathrm{E}-04$ & $6.579 \mathrm{E}-02$ & $4.678 \mathrm{E}+04$ \\
\hline & 131187.190 & 144142.160 & $1.180 \mathrm{E}-01$ & 6 & 4 & $3.081 \mathrm{E}-05$ & $4.699 \mathrm{E}-03$ & $5.168 \mathrm{E}+03$ \\
\hline & 130641.110 & 144009.420 & $1.218 \mathrm{E}-01$ & 4 & 6 & $4.770 \mathrm{E}-05$ & $4.699 E-03$ & $3.789 \mathrm{E}+03$ \\
\hline & 130641.110 & 144142.160 & $1.230 \mathrm{E}-01$ & 4 & 4 & $4.335 \mathrm{E}-04$ & $4.229 \mathrm{E}-02$ & $5.268 \mathrm{E}+04$ \\
\hline \multirow[t]{5}{*}{$y^{2} D^{\circ} \rightarrow e^{2} D^{e}$} & 0.5272 & 0.3639 & $1.633 E-01$ & 10 & 10 & $5.853 \mathrm{E}-02$ & $1.075 \mathrm{E}+01$ & $1.254 \mathrm{E}+07$ \\
\hline & 131187.190 & 148886.570 & $1.613 E-01$ & 6 & 6 & $5.395 \mathrm{E}-02$ & $6.020 \mathrm{E}+00$ & $1.127 \mathrm{E}+07$ \\
\hline & 131187.190 & 148900.910 & $1.614 \mathrm{E}-01$ & 6 & 4 & $3.856 \mathrm{E}-03$ & $4.300 \mathrm{E}-01$ & $1.210 \mathrm{E}+06$ \\
\hline & 130641.110 & 148886.570 & $1.663 \mathrm{E}-01$ & 4 & 6 & $5.959 \mathrm{E}-03$ & $4.300 \mathrm{E}-01$ & $8.825 \mathrm{E}+05$ \\
\hline & 130641.110 & 148900.910 & $1.664 \mathrm{E}-01$ & 4 & 4 & $5.366 \mathrm{E}-02$ & $3.870 \mathrm{E}+00$ & $1.194 \mathrm{E}+07$ \\
\hline \multirow[t]{5}{*}{$y^{2} D^{\circ} \rightarrow f^{2} D^{e}$} & 0.5272 & 0.2673 & $2.599 \mathrm{E}-01$ & 10 & 10 & $2.724 \mathrm{E}-02$ & $3.144 E+\infty$ & $1.479 \mathrm{E}+07$ \\
\hline & 131187.190 & 159492.830 & $2.579 \mathrm{E}-01$ & 6 & 6 & $2.523 \mathrm{E}-02$ & $1.761 \mathrm{E}+\infty 0$ & $1.348 \mathrm{E}+07$ \\
\hline & 131187.190 & 159495.940 & $2.579 \mathrm{E}-01$ & 6 & 4 & $1.802 E-03$ & $1.258 \mathrm{E}-01$ & $1.444 E+06$ \\
\hline & 130641.110 & 159492.830 & $2.629 \mathrm{E}-01$ & 4 & 6 & $2.755 E-03$ & $1.258 \mathrm{E}-01$ & $1.020 \mathrm{E}+06$ \\
\hline & 130641.110 & 159495.940 & $2.629 E-01$ & 4 & 4 & $2.480 \mathrm{E}-02$ & $1.132 \mathrm{E}+\infty$ & $1.377 \mathrm{E}+07$ \\
\hline \multirow[t]{5}{*}{$y^{2} D^{\circ} \rightarrow g^{2} D^{e}$} & 0.5272 & 0.2469 & $2.804 \mathrm{E}-01$ & 10 & 10 & $1.454 \mathrm{E}-02$ & $1.556 \mathrm{E}+\infty 0$ & $9.179 E+06$ \\
\hline & 131187.190 & 161733.100 & $2.783 \mathrm{E}-01$ & 6 & 6 & $1.347 \mathrm{E}-02$ & $8.712 \mathrm{E}-01$ & $8.379 \mathrm{E}+06$ \\
\hline & 131187.190 & 161737.990 & $2.784 \mathrm{E}-01$ & 6 & 4 & $9.624 \mathrm{E}-04$ & $6.223 \mathrm{E}-02$ & $8.987 \mathrm{E}+05$ \\
\hline & 130641.110 & 161733.100 & $2.833 E-01$ & 4 & 6 & $1.469 E-03$ & $6.223 \mathrm{E}-02$ & $6.314 \mathrm{E}+05$ \\
\hline & 130641.110 & 161737.990 & $2.834 \mathrm{E}-01$ & 4 & 4 & $1.323 \mathrm{E}-02$ & $5.600 \mathrm{E}-01$ & $8.532 E+06$ \\
\hline \multirow[t]{5}{*}{$\mathrm{y}^{2} \mathrm{D}^{\circ} \rightarrow \mathrm{b}^{2} \mathrm{D}^{\circ}$} & 0.5272 & 0.1944 & $3.328 \mathrm{E}-01$ & 10 & 10 & $5.236 \mathrm{E}-03$ & $4.720 \mathrm{E}-01$ & $4.659 \mathrm{E}+06$ \\
\hline & 131187.190 & 167506.390 & $3.309 \mathrm{E}-01$ & 6 & 6 & $4.859 \mathrm{E}-03$ & $2.643 \mathrm{E}-01$ & $4.273 E+06$ \\
\hline & 131187.190 & 167472.420 & $3.306 \mathrm{E}-01$ & 6 & 4 & $3.467 \mathrm{E}-04$ & $1.888 \mathrm{E}-02$ & $4.566 \mathrm{E}+05$ \\
\hline & 130641.110 & 167506.390 & $3.359 \mathrm{E}-01$ & 4 & 6 & $5.284 \mathrm{E}-04$ & $1.888 E-02$ & $3.193 E+05$ \\
\hline & 130641.110 & 167472.420 & $3.356 \mathrm{E}-01$ & 4 & 4 & $4.752 \mathrm{E}-03$ & $1.699 \mathrm{E}-01$ & $4.299 \mathrm{E}+06$ \\
\hline \multirow[t]{5}{*}{$y^{2} D^{o} \rightarrow i^{2} D^{e}$} & 0.5272 & 0.1717 & $3.555 \mathrm{E}-01$ & 10 & 10 & $2.077 \mathrm{E}-02$ & $1.753 \mathrm{E}+\infty$ & $2.109 E+07$ \\
\hline & 131187.190 & 170015.230 & $3.538 E-01$ & 6 & 6 & $1.930 \mathrm{E}-02$ & $9.818 \mathrm{E}-01$ & $1.940 \mathrm{E}+07$ \\
\hline & 131187.190 & 169922.270 & $3.529 \mathrm{E}-01$ & 6 & 4 & $1.375 E-03$ & $7.013 \mathrm{E}-02$ & $2.063 E+06$ \\
\hline & 130641.110 & 170015.230 & $3.588 \mathrm{E}-01$ & 4 & 6 & $2.097 \mathrm{E}-03$ & $7.013 E-02$ & $1.445 \mathrm{E}+06$ \\
\hline & 130641.110 & 169922.270 & $3.579 \mathrm{E}-01$ & 4 & 4 & $1.882 \mathrm{E}-02$ & $6.311 \mathrm{E}-01$ & $1.937 \mathrm{E}+07$ \\
\hline
\end{tabular}


Table IV. Continued

\begin{tabular}{|c|c|c|c|c|c|c|c|c|}
\hline Transition & $\begin{array}{l}E_{i} \\
\mathrm{~cm}^{-1} \\
\end{array}$ & $\begin{array}{l}E_{f} \\
\mathrm{~cm}^{-1}\end{array}$ & $\begin{array}{l}E_{f i} \\
\mathrm{Ry} \\
\end{array}$ & $g_{i}$ & $g_{f}$ & $f_{i f}$ & $S$ & $\begin{array}{l}A_{f i} \\
\mathrm{~s}^{-1} \\
\end{array}$ \\
\hline \multirow[t]{5}{*}{$\mathrm{y}^{2} \mathrm{D}^{\circ} \rightarrow \mathrm{j}^{2} \mathrm{D}^{\circ}$} & 0.5272 & 0.1071 & $4.202 \mathrm{E}-01$ & 10 & 10 & $2.607 \mathrm{E}-03$ & $1.861 \mathrm{E}-01$ & $3.696 \mathrm{E}+06$ \\
\hline & 131187.190 & 177076.280 & $4.181 \mathrm{E}-01$ & 6 & 6 & $2.421 \mathrm{E}-03$ & $1.042 \mathrm{E}-01$ & $3.399 \mathrm{E}+06$ \\
\hline & 131187.190 & 177077.020 & $4.181 \mathrm{E}-01$ & 6 & 4 & $1.729 \mathrm{E}-04$ & $7.444 \mathrm{E}-03$ & $3.642 \mathrm{E}+05$ \\
\hline & 130641.110 & 177076.280 & $4.231 \mathrm{E}-01$ & 4 & 6 & $2.625 E-04$ & $7.444 \mathrm{E}-03$ & $2.516 \mathrm{E}+05$ \\
\hline & 130641.110 & 177077.020 & $4.231 \mathrm{E}-01$ & 4 & 4 & $2.362 \mathrm{E}-03$ & $6.700 \mathrm{E}-02$ & $3.397 \mathrm{E}+06$ \\
\hline \multirow[t]{5}{*}{$\mathrm{y}^{2} \mathrm{D}^{\circ} \rightarrow \mathrm{k}^{2} \mathrm{D}^{\mathrm{e}}$} & 0.5272 & 0.0381 & $4.891 \mathrm{E}-01$ & 10 & 10 & $8.981 \mathrm{E}-04$ & $5.508 \mathrm{E}-02$ & $1.726 \mathrm{E}+06$ \\
\hline & 131187.190 & 184642.760 & $4.871 \mathrm{E}-01$ & 6 & 6 & $8.348 E-04$ & $3.085 \mathrm{E}-02$ & $1.591 \mathrm{E}+06$ \\
\hline & 131187.190 & 184642.760 & $4.871 \mathrm{E}-01$ & 6 & 4 & $5.963 E-05$ & $2.203 E-03$ & $1.704 \mathrm{E}+05$ \\
\hline & 130641.110 & 184642.760 & $4.921 \mathrm{E}-01$ & 4 & 6 & $9.036 \mathrm{E}-05$ & $2.203 \mathrm{E}-03$ & $1.172 \mathrm{E}+05$ \\
\hline & 130641.110 & 184642.760 & $4.921 \mathrm{E}-01$ & 4 & 4 & $8.132 E-04$ & $1.983 \mathrm{E}-02$ & $1.582 \mathrm{E}+06$ \\
\hline \multirow[t]{4}{*}{$\mathrm{y}^{2} \mathrm{D}^{\circ} \rightarrow \mathrm{b}^{2} \mathrm{~F}^{\mathrm{c}}$} & 0.5272 & 0.4584 & $6.881 \mathrm{E}-02$ & 10 & 14 & $1.116 \mathrm{E}-01$ & $4.864 \mathrm{E}+01$ & $3.031 E+06$ \\
\hline & 131187.190 & 138527.980 & $6.690 \mathrm{E}-02$ & 6 & 8 & $1.033 E-01$ & $2.779 \mathrm{E}+01$ & $2.785 E+06$ \\
\hline & 131187.190 & 138509.170 & $6.670 \mathrm{E}-02$ & 6 & 6 & $5.149 \mathrm{E}-03$ & $1.390 \mathrm{E}+00$ & $1.840 \mathrm{E}+05$ \\
\hline & 130641.110 & 138509.170 & $7.170 \mathrm{E}-02$ & 4 & 6 & $1.162 \mathrm{E}-01$ & $1.946 \mathrm{E}+01$ & $3.200 \mathrm{E}+06$ \\
\hline \multirow[t]{4}{*}{$y^{2} D^{\circ} \rightarrow c^{2} F^{e}$} & 0.5272 & 0.2955 & $2.317 \mathrm{E}-01$ & 10 & 14 & $6.195 \mathrm{E}-01$ & $8.021 \mathrm{E}+01$ & $1.909 \mathrm{E}+08$ \\
\hline & 131187.190 & 156604.170 & $2.316 \mathrm{E}-01$ & 6 & 8 & $5.897 \mathrm{E}-01$ & $4.583 \mathrm{E}+01$ & $1.906 \mathrm{E}+08$ \\
\hline & 131187.190 & 156121.700 & $2.272 \mathrm{E}-01$ & 6 & 6 & $2.893 \mathrm{E}-02$ & $2.292 \mathrm{E}+00$ & $1.199 \mathrm{E}+07$ \\
\hline & 130641.110 & 156121.700 & $2.322 \mathrm{E}-01$ & 4 & 6 & $6.208 \mathrm{E}-01$ & $3.208 \mathrm{E}+01$ & $1.792 \mathrm{E}+08$ \\
\hline \multirow[t]{4}{*}{$\mathrm{y}^{2} \mathrm{D}^{\circ} \rightarrow \mathrm{d}^{2} \mathrm{~F}^{\mathrm{e}}$} & 0.5272 & 0.2236 & $3.037 \mathrm{E}-01$ & 10 & 14 & $2.187 \mathrm{E}-02$ & $2.161 \mathrm{E}+00$ & $1.157 \mathrm{E}+07$ \\
\hline & 131187.190 & 164337.610 & $3.021 \mathrm{E}-01$ & 6 & 8 & $2.072 \mathrm{E}-02$ & $1.235 \mathrm{E}+\infty 0$ & $1.139 \mathrm{E}+07$ \\
\hline & 131187.190 & 164232.360 & $3.011 \mathrm{E}-01$ & 6 & 6 & $1.033 \mathrm{E}-03$ & $6.173 \mathrm{E}-02$ & $7.520 \mathrm{E}+05$ \\
\hline & 130641.110 & 164232.360 & $3.061 \mathrm{E}-01$ & 4 & 6 & $2.205 \mathrm{E}-02$ & $8.643 E-01$ & $1.106 \mathrm{E}+07$ \\
\hline \multirow[t]{4}{*}{$\mathrm{y}^{2} \mathrm{D}^{\circ} \rightarrow \mathrm{e}^{2} \mathrm{~F}^{\mathrm{e}}$} & 0.5272 & 0.1759 & $3.513 \mathrm{E}-01$ & 10 & 14 & $5.681 \mathrm{E}-02$ & $4.851 \mathrm{E}+\infty 0$ & $4.023 \mathrm{E}+07$ \\
\hline & 131187.190 & 169702.320 & $3.509 \mathrm{E}-01$ & 6 & 8 & $5.404 \mathrm{E}-02$ & $2.772 \mathrm{E}+00$ & $4.009 \mathrm{E}+07$ \\
\hline & 131187.190 & 169283.540 & $3.471 \mathrm{E}-01$ & 6 & 6 & $2.673 \mathrm{E}-03$ & $1.386 \mathrm{E}-01$ & $2.586 \mathrm{E}+06$ \\
\hline & 130641.110 & 169283.540 & $3.521 \mathrm{E}-01$ & 4 & 6 & $5.694 \mathrm{E}-02$ & $1.940 \mathrm{E}+00$ & $3.780 \mathrm{E}+07$ \\
\hline \multirow[t]{4}{*}{$\mathrm{x}^{2} \mathrm{D}^{\circ} \rightarrow \mathrm{d}^{2} \mathrm{P}^{\mathrm{e}}$} & 0.4383 & 0.3936 & $4.469 \mathrm{E}-02$ & 10 & 6 & $8.509 \mathrm{E}-04$ & $5.712 \mathrm{E}-01$ & $2.275 \mathrm{E}+04$ \\
\hline & 140708.890 & 145505.740 & $4.370 \mathrm{E}-02$ & 6 & 4 & $8.320 \mathrm{E}-04$ & $3.427 \mathrm{E}-01$ & $1.914 \mathrm{E}+04$ \\
\hline & 140750.340 & 145505.740 & $4.330 \mathrm{E}-02$ & 4 & 4 & $1.374 \mathrm{E}-04$ & $3.808 \mathrm{E}-02$ & $2.069 E+03$ \\
\hline & 140750.340 & 145877.660 & $4.670 \mathrm{E}-02$ & 4 & 2 & $7.409 E-04$ & $1.904 \mathrm{E}-01$ & $2.596 \mathrm{E}+04$ \\
\hline \multirow[t]{4}{*}{$\mathrm{x}^{2} \mathrm{D}^{\circ} \rightarrow \mathrm{e}^{2} \mathrm{P}^{\mathrm{e}}$} & 0.4383 & 0.3380 & $1.003 \mathrm{E}-01$ & 10 & 6 & $4.431 \mathrm{E}-02$ & $1.325 \mathrm{E}+01$ & $5.970 \mathrm{E}+06$ \\
\hline & 140708.890 & 151910.830 & $1.021 \mathrm{E}-01$ & 6 & 4 & $4.509 \mathrm{E}-02$ & $7.949 \mathrm{E}+\infty 0$ & $5.663 \mathrm{E}+06$ \\
\hline & 140750.340 & 151910.830 & $1.017 \mathrm{E}-01$ & 4 & 4 & $7.486 \mathrm{E}-03$ & $8.833 \mathrm{E}-01$ & $6.219 \mathrm{E}+05$ \\
\hline & 140750.340 & 151383.810 & $9.690 \mathrm{E}-02$ & 4 & 2 & $3.566 \mathrm{E}-02$ & $4.416 \mathrm{E}+\infty$ & $5.379 \mathrm{E}+06$ \\
\hline \multirow[t]{4}{*}{$\mathrm{x}^{2} \mathrm{D}^{\circ} \rightarrow \mathrm{f}^{2} \mathrm{P}^{\circ}$} & 0.4383 & 0.2720 & $1.663 \mathrm{E}-01$ & 10 & 6 & $2.952 \mathrm{E}-03$ & $5.324 \mathrm{E}-01$ & $1.094 \mathrm{E}+06$ \\
\hline & 140708.890 & 158828.310 & $1.652 \mathrm{E}-01$ & 6 & 4 & $2.932 \mathrm{E}-03$ & $3.195 \mathrm{E}-01$ & $9.640 \mathrm{E}+05$ \\
\hline & 140750.340 & 158828.310 & $1.648 \mathrm{E}-01$ & 4 & 4 & $4.875 \mathrm{E}-04$ & $3.549 \mathrm{E}-02$ & $1.063 \mathrm{E}+05$ \\
\hline & 140750.340 & 159283.660 & $1.689 \mathrm{E}-01$ & 4 & 2 & $2.498 \mathrm{E}-03$ & $1.775 \mathrm{E}-01$ & $1.145 \mathrm{E}+06$ \\
\hline \multirow[t]{4}{*}{$\mathrm{x}^{2} \mathrm{D}^{\circ} \rightarrow \mathrm{g}^{2} \mathrm{P}^{\mathrm{e}}$} & 0.4383 & 0.2064 & $2.319 \mathrm{E}-01$ & 10 & 6 & $3.155 \mathrm{E}-02$ & $4.081 \mathrm{E}+\infty 0$ & $2.271 \mathrm{E}+07$ \\
\hline & 140708.890 & 166185.470 & $2.322 \mathrm{E}-01$ & 6 & 4 & $3.159 \mathrm{E}-02$ & $2.449 \mathrm{E}+00$ & $2.052 \mathrm{E}+07$ \\
\hline & 140750.340 & 166185.470 & $2.318 \mathrm{E}-01$ & 4 & 4 & $5.256 \mathrm{E}-03$ & $2.721 \mathrm{E}-01$ & $2.268 \mathrm{E}+06$ \\
\hline & 140750.340 & 166151.610 & $2.315 \mathrm{E}-01$ & 4 & 2 & $2.625 \mathrm{E}-02$ & $1.360 \mathrm{E}+00$ & $2.260 \mathrm{E}+07$ \\
\hline \multirow[t]{4}{*}{$\mathrm{x}^{2} \mathrm{D}^{\circ} \rightarrow \mathrm{b}^{2} \mathrm{P}^{\mathrm{e}}$} & 0.4383 & 0.2017 & $2.366 \mathrm{E}-01$ & 10 & 6 & $1.970 \mathrm{E}-02$ & $2.498 \mathrm{E}+00$ & $1.476 \mathrm{E}+07$ \\
\hline & 140708.890 & 166862.710 & $2.384 \mathrm{E}-01$ & 6 & 4 & $1.985 \mathrm{E}-02$ & $1.499 \mathrm{E}+00$ & $1.359 E+07$ \\
\hline & 140750.340 & 166862.710 & $2.380 \mathrm{E}-01$ & 4 & 4 & $3.303 \mathrm{E}-03$ & $1.665 \mathrm{E}-01$ & $1.503 \mathrm{E}+06$ \\
\hline & 140750.340 & 166331.190 & $2.331 \mathrm{E}-01$ & 4 & 2 & $1.617 \mathrm{E}-02$ & $8.326 \mathrm{E}-01$ & $1.412 \mathrm{E}+07$ \\
\hline
\end{tabular}


Table IV. Continued

\begin{tabular}{|c|c|c|c|c|c|c|c|c|}
\hline Transition & $\begin{array}{l}E_{i} \\
\mathrm{~cm}^{-1}\end{array}$ & $\begin{array}{l}E_{f} \\
\mathrm{~cm}^{-1}\end{array}$ & $\begin{array}{l}E_{f i} \\
\text { Ry }\end{array}$ & $g_{i}$ & $g_{f}$ & $f_{i f}$ & $S$ & $\begin{array}{l}A_{f i} \\
\mathrm{~s}^{-1} \\
\end{array}$ \\
\hline \multirow[t]{4}{*}{$\mathrm{x}^{2} \mathrm{D}^{\circ} \rightarrow \mathrm{i}^{2} \mathrm{P}^{\circ}$} & 0.4383 & 0.1765 & $2.618 \mathrm{E}-01$ & 10 & 6 & $3.946 \mathrm{E}-03$ & $4.522 \mathrm{E}-01$ & $3.621 E+06$ \\
\hline & 140708.890 & 169340.390 & $2.609 \mathrm{E}-01$ & 6 & 4 & $3.933 \mathrm{E}-03$ & $2.713 \mathrm{E}-01$ & $3.225 \mathrm{E}+06$ \\
\hline & 140750.340 & 169340.390 & $2.605 \mathrm{E}-01$ & 4 & 4 & $6.544 \mathrm{E}-04$ & $3.015 \mathrm{E}-02$ & $3.567 \mathrm{E}+05$ \\
\hline & 140750.340 & 169687.520 & $2.637 \mathrm{E}-01$ & 4 & 2 & $3.312 \mathrm{E}-03$ & $1.507 \mathrm{E}-01$ & $3.700 \mathrm{E}+06$ \\
\hline \multirow[t]{4}{*}{$\mathrm{x}^{2} \mathrm{D}^{\circ} \rightarrow \mathrm{j}^{2} \mathrm{P}^{\mathrm{e}}$} & 0.4383 & 0.1342 & $3.041 \mathrm{E}-01$ & 10 & 6 & $2.759 \mathrm{E}-04$ & $2.722 E-02$ & $3.416 \mathrm{E}+05$ \\
\hline & 140708.890 & 174274.520 & $3.059 \mathrm{E}-01$ & 6 & 4 & $2.775 \mathrm{E}-04$ & $1.633 E-02$ & $3.129 \mathrm{E}+05$ \\
\hline & 140750.340 & 174274.520 & $3.055 \mathrm{E}-01$ & 4 & 4 & $4.619 \mathrm{E}-05$ & $1.814 \mathrm{E}-03$ & $3.463 E+04$ \\
\hline & 140750.340 & 173749.010 & $3.007 \mathrm{E}-01$ & 4 & 2 & $2.273 \mathrm{E}-04$ & $9.072 \mathrm{E}-03$ & $3.302 \mathrm{E}+05$ \\
\hline \multirow[t]{5}{*}{$\mathrm{x}^{2} \mathrm{D}^{\circ} \rightarrow \mathrm{d}^{2} \mathrm{D}^{\mathrm{e}}$} & 0.4383 & 0.4079 & $3.041 E-02$ & 10 & 10 & $2.462 \mathrm{E}-02$ & $2.429 \mathrm{E}+01$ & $1.829 \mathrm{E}+05$ \\
\hline & 140708.890 & 144009.420 & $3.010 \mathrm{E}-02$ & 6 & 6 & $2.275 \mathrm{E}-02$ & $1.360 \mathrm{E}+01$ & $1.655 \mathrm{E}+05$ \\
\hline & 140708.890 & 144142.160 & $3.130 \mathrm{E}-02$ & 6 & 4 & $1.690 \mathrm{E}-03$ & $9.717 \mathrm{E}-01$ & $1.994 \mathrm{E}+04$ \\
\hline & 140750.340 & 144009.420 & $2.970 \mathrm{E}-02$ & 4 & 6 & $2.405 \mathrm{E}-03$ & $9.717 \mathrm{E}-01$ & $1.136 \mathrm{E}+04$ \\
\hline & 140750.340 & 144142.160 & $3.090 E-02$ & 4 & 4 & $2.252 \mathrm{E}-02$ & $8.745 E+00$ & $1.727 \mathrm{E}+05$ \\
\hline \multirow[t]{5}{*}{$x^{2} D^{\circ} \rightarrow e^{2} D^{e}$} & 0.4383 & 0.3639 & $7.442 \mathrm{E}-02$ & 10 & 10 & $4.783 E-03$ & $1.928 \mathrm{E}+\infty$ & $2.128 E+05$ \\
\hline & 140708.890 & 148886.570 & $7.460 \mathrm{E}-02$ & 6 & 6 & $4.475 \mathrm{E}-03$ & $1.080 \mathrm{E}+00$ & $2.000 \mathrm{E}+05$ \\
\hline & 140708.890 & 148900.910 & $7.470 \mathrm{E}-02$ & 6 & 4 & $3.201 \mathrm{E}-04$ & $7.713 E-02$ & $2.152 E+04$ \\
\hline & 140750.340 & 148886.570 & $7.420 \mathrm{E}-02$ & 4 & 6 & $4.769 \mathrm{E}-04$ & $7.713 E-02$ & $1.406 \mathrm{E}+04$ \\
\hline & 140750.340 & 148900.910 & $7.430 \mathrm{E}-02$ & 4 & 4 & $4.298 \mathrm{E}-03$ & $6.941 \mathrm{E}-01$ & $1.906 \mathrm{E}+05$ \\
\hline \multirow[t]{5}{*}{$x^{2} D^{\circ} \rightarrow f^{2} D^{e}$} & 0.4383 & 0.2673 & $1.710 \mathrm{E}-01$ & 10 & 10 & $2.871 \mathrm{E}-02$ & $5.035 E+\infty 0$ & $6.745 \mathrm{E}+06$ \\
\hline & 140708.890 & 159492.830 & $1.712 \mathrm{E}-01$ & 6 & 6 & $2.682 \mathrm{E}-02$ & $2.820 \mathrm{E}+00$ & $6.314 \mathrm{E}+06$ \\
\hline & 140708.890 & 159495.940 & $1.712 \mathrm{E}-01$ & 6 & 4 & $1.916 \mathrm{E}-03$ & $2.014 \mathrm{E}-01$ & $6.765 \mathrm{E}+05$ \\
\hline & 140750.340 & 159492.830 & $1.708 \mathrm{E}-01$ & 4 & 6 & $2.867 \mathrm{E}-03$ & $2.014 \mathrm{E}-01$ & $4.478 \mathrm{E}+05$ \\
\hline & 140750.340 & 159495.940 & $1.708 \mathrm{E}-01$ & 4 & 4 & $2.580 \mathrm{E}-02$ & $1.813 \mathrm{E}+\infty 0$ & $6.046 \mathrm{E}+06$ \\
\hline \multirow[t]{5}{*}{$x^{2} D^{\circ} \rightarrow g^{2} D^{e}$} & 0.4383 & 0.2469 & $1.915 \mathrm{E}-01$ & 10 & 10 & $2.646 \mathrm{E}-01$ & $4.146 \mathrm{E}+01$ & $7.791 \mathrm{E}+07$ \\
\hline & 140708.890 & 161733.100 & $1.916 \mathrm{E}-01$ & 6 & 6 & $2.472 \mathrm{E}-01$ & $2.322 \mathrm{E}+01$ & $7.288 \mathrm{E}+07$ \\
\hline & 140708.890 & 161737.990 & $1.917 \mathrm{E}-01$ & 6 & 4 & $1.766 \mathrm{E}-02$ & $1.659 \mathrm{E}+\infty 0$ & $7.821 \mathrm{E}+06$ \\
\hline & 140750.340 & 161733.100 & $1.912 \mathrm{E}-01$ & 4 & 6 & $2.643 \mathrm{E}-02$ & $1.659 \mathrm{E}+00$ & $5.173 \mathrm{E}+06$ \\
\hline & 140750.340 & 161737.990 & $1.913 \mathrm{E}-01$ & 4 & 4 & $2.380 \mathrm{E}-01$ & $1.493 \mathrm{E}+01$ & $6.995 E+07$ \\
\hline \multirow[t]{5}{*}{$\mathrm{x}^{2} \mathrm{D}^{\circ} \rightarrow \mathrm{h}^{2} \mathrm{D}^{e}$} & 0.4383 & 0.1944 & $2.439 \mathrm{E}-01$ & 10 & 10 & $1.652 \mathrm{E}-01$ & $2.031 \mathrm{E}+01$ & $7.893 \mathrm{E}+07$ \\
\hline & 140708.890 & 167506.390 & $2.442 \mathrm{E}-01$ & 6 & 6 & $1.543 \mathrm{E}-01$ & $1.137 \mathrm{E}+01$ & $7.392 \mathrm{E}+07$ \\
\hline & 140708.890 & 167472.420 & $2.439 \mathrm{E}-01$ & 6 & 4 & $1.101 \mathrm{E}-02$ & $8.125 \mathrm{E}-01$ & $7.890 \mathrm{E}+06$ \\
\hline & 140750.340 & 167506.390 & $2.438 \mathrm{E}-01$ & 4 & 6 & $1.651 \mathrm{E}-02$ & $8.125 \mathrm{E}-01$ & $5.254 \mathrm{E}+06$ \\
\hline & 140750.340 & 167472.420 & $2.435 \mathrm{E}-01$ & 4 & 4 & $1.484 \mathrm{E}-01$ & $7.312 E+00$ & $7.067 \mathrm{E}+07$ \\
\hline \multirow[t]{5}{*}{$\mathrm{x}^{2} \mathrm{D}^{\circ} \rightarrow \mathrm{i}^{2} \mathrm{D}^{\circ}$} & 0.4383 & 0.1717 & $2.666 \mathrm{E}-01$ & 10 & 10 & $3.177 \mathrm{E}-03$ & $3.575 \mathrm{E}-01$ & $1.813 \mathrm{E}+06$ \\
\hline & 140708.890 & 170015.230 & $2.671 \mathrm{E}-01$ & 6 & 6 & $2.971 \mathrm{E}-03$ & $2.002 \mathrm{E}-01$ & $1.702 \mathrm{E}+06$ \\
\hline & 140708.890 & 169922.270 & $2.662 \mathrm{E}-01$ & 6 & 4 & $2.115 \mathrm{E}-04$ & $1.430 \mathrm{E}-02$ & $1.806 \mathrm{E}+05$ \\
\hline & 140750.340 & 170015.230 & $2.667 \mathrm{E}-01$ & 4 & 6 & $3.178 E-04$ & $1.430 \mathrm{E}-02$ & $1.211 E+05$ \\
\hline & 140750.340 & 169922.270 & $2.658 \mathrm{E}-01$ & 4 & 4 & $2.851 \mathrm{E}-03$ & $1.287 \mathrm{E}-01$ & $1.618 \mathrm{E}+06$ \\
\hline \multirow[t]{5}{*}{$x^{2} D^{\circ} \rightarrow j^{2} D^{e}$} & 0.4383 & 0.1071 & $3.313 \mathrm{E}-01$ & 10 & 10 & $2.574 \mathrm{E}-02$ & $2.331 \mathrm{E}+00$ & $2.269 \mathrm{E}+07$ \\
\hline & 140708.890 & 177076.280 & $3.314 \mathrm{E}-01$ & 6 & 6 & $2.403 \mathrm{E}-02$ & $1.305 \mathrm{E}+00$ & $2.120 \mathrm{E}+07$ \\
\hline & 140708.890 & 177077.020 & $3.314 \mathrm{E}-01$ & 6 & 4 & $1.717 \mathrm{E}-03$ & $9.325 \mathrm{E}-02$ & $2.272 E+06$ \\
\hline & 140750.340 & 177076.280 & $3.310 \mathrm{E}-01$ & 4 & 6 & $2.572 \mathrm{E}-03$ & $9.325 \mathrm{E}-02$ & $1.509 \mathrm{E}+06$ \\
\hline & 140750.340 & 177077.020 & $3.310 \mathrm{E}-01$ & 4 & 4 & $2.315 \mathrm{E}-02$ & $8.392 \mathrm{E}-01$ & $2.037 \mathrm{E}+07$ \\
\hline \multirow[t]{5}{*}{$x^{2} D^{\circ} \rightarrow k^{2} D^{e}$} & 0.4383 & 0.0381 & $4.002 \mathrm{E}-01$ & 10 & 10 & $7.928 \mathrm{E}-05$ & $5.943 \mathrm{E}-03$ & $1.020 \mathrm{E}+05$ \\
\hline & 140708.890 & 184642.760 & $4.004 \mathrm{E}-01$ & 6 & 6 & $7.403 \mathrm{E}-05$ & $3.328 \mathrm{E}-03$ & $9.533 \mathrm{E}+04$ \\
\hline & 140708.890 & 184642.760 & $4.004 \mathrm{E}-01$ & 6 & 4 & $5.288 \mathrm{E}-06$ & $2.377 \mathrm{E}-04$ & $1.021 E+04$ \\
\hline & 140750.340 & 184642.760 & $4.000 E-01$ & 4 & 6 & $7.924 \mathrm{E}-06$ & $2.377 \mathrm{E}-04$ & $6.789 E+03$ \\
\hline & 140750.340 & 184642.760 & $4.000 \mathrm{E}-01$ & 4 & 4 & $7.131 E-05$ & $2.139 \mathrm{E}-03$ & $9.165 E+04$ \\
\hline
\end{tabular}


Table IV. Continued

\begin{tabular}{|c|c|c|c|c|c|c|c|c|}
\hline Transition & $\begin{array}{l}E_{i} \\
\mathrm{~cm}^{-1}\end{array}$ & $\begin{array}{l}E_{f} \\
\mathrm{~cm}^{-1} \\
\end{array}$ & $\begin{array}{l}E_{f i} \\
\text { Ry }\end{array}$ & $g_{i}$ & $g_{f}$ & $f_{i f}$ & $S$ & $\begin{array}{l}A_{f i} \\
\mathrm{~s}^{-1}\end{array}$ \\
\hline \multirow[t]{4}{*}{$\mathrm{x}^{2} \mathrm{D}^{\circ} \rightarrow \mathrm{c}^{2} \mathrm{~F}^{0}$} & 0.4383 & 0.2955 & $1.428 \mathrm{E}-01$ & 10 & 14 & $5.319 \mathrm{E}-04$ & $1.117 \mathrm{E}-01$ & $6.224 \mathrm{E}+04$ \\
\hline & 140708.890 & 156604.170 & $1.449 \mathrm{E}-01$ & 6 & 8 & $5.140 \mathrm{E}-04$ & $6.385 \mathrm{E}-02$ & $6.501 \mathrm{E}+04$ \\
\hline & 140708.890 & 156121.700 & $1.405 \mathrm{E}-01$ & 6 & 6 & $2.492 \mathrm{E}-05$ & $3.192 \mathrm{E}-03$ & $3.951 \mathrm{E}+03$ \\
\hline & 140750.340 & 156121.700 & $1.401 \mathrm{E}-01$ & 4 & 6 & $5.218 \mathrm{E}-04$ & $4.469 \mathrm{E}-02$ & $5.484 \mathrm{E}+04$ \\
\hline \multirow[t]{4}{*}{$\mathrm{x}^{2} \mathrm{D}^{\circ} \rightarrow \mathrm{d}^{2} \mathrm{~F}^{\mathrm{e}}$} & 0.4383 & 0.2236 & $2.148 \mathrm{E}-01$ & 10 & 14 & $4.520 \mathrm{E}-01$ & $6.314 \mathrm{E}+01$ & $1.196 \mathrm{E}+08$ \\
\hline & 140708.890 & 164337.610 & $2.154 \mathrm{E}-01$ & 6 & 8 & $4.318 \mathrm{E}-01$ & $3.608 \mathrm{E}+01$ & $1.207 \mathrm{E}+08$ \\
\hline & 140708.890 & 164232.360 & $2.144 \mathrm{E}-01$ & 6 & 6 & $2.149 \mathrm{E}-02$ & $1.804 \mathrm{E}+\infty 0$ & $7.934 \mathrm{E}+06$ \\
\hline & 140750.340 & 164232.360 & $2.140 \mathrm{E}-01$ & 4 & 6 & $4.504 \mathrm{E}-01$ & $2.526 \mathrm{E}+01$ & $1.105 \mathrm{E}+08$ \\
\hline \multirow{4}{*}{$x^{2} D^{\circ} \rightarrow e^{2} F^{e}$} & 0.4383 & 0.1759 & $2.624 \mathrm{E}-01$ & 10 & 14 & $8.317 \mathrm{E}-02$ & $9.508 \mathrm{E}+00$ & $3.286 \mathrm{E}+07$ \\
\hline & 140708.890 & 169702.320 & $2.642 \mathrm{E}-01$ & 6 & 8 & $7.975 \mathrm{E}-02$ & $5.433 \mathrm{E}+00$ & $3.353 \mathrm{E}+07$ \\
\hline & 140708.890 & 169283.540 & $2.604 E-01$ & 6 & 6 & $3.930 \mathrm{E}-03$ & $2.717 \mathrm{E}-01$ & $2.141 \mathrm{E}+06$ \\
\hline & 140750.340 & 169283.540 & $2.600 \mathrm{E}-01$ & 4 & 6 & $8.240 \mathrm{E}-02$ & $3.803 E+00$ & $2.983 \mathrm{E}+07$ \\
\hline \multirow[t]{4}{*}{$w^{2} D^{\circ} \rightarrow f^{2} P^{\circ}$} & 0.2762 & 0.2720 & $4.234 \mathrm{E}-03$ & 10 & 6 & $5.873 \mathrm{E}-04$ & $4.162 \mathrm{E}+00$ & $1.409 \mathrm{E}+02$ \\
\hline & 158715.460 & 158828.310 & $1.100 \mathrm{E}-03$ & 6 & 4 & $1.526 \mathrm{E}-04$ & $2.497 \mathrm{E}+00$ & $2.225 \mathrm{E}+00$ \\
\hline & 158215.590 & 158828.310 & $5.600 \mathrm{E}-03$ & 4 & 4 & $1.295 \mathrm{E}-04$ & $2.775 \mathrm{E}-01$ & $3.261 \mathrm{E}+01$ \\
\hline & 158215.590 & 159283.660 & $9.700 \mathrm{E}-03$ & 4 & 2 & $1.121 \mathrm{E}-03$ & $1.387 \mathrm{E}+00$ & $1.695 \mathrm{E}+03$ \\
\hline \multirow{4}{*}{$w^{2} D^{\circ} \rightarrow g^{2} P^{e}$} & 0.2762 & 0.2064 & $6.979 \mathrm{E}-02$ & 10 & 6 & $1.878 \mathrm{E}-01$ & $8.074 \mathrm{E}+01$ & $1.225 \mathrm{E}+07$ \\
\hline & 158715.460 & 166185.470 & $6.810 \mathrm{E}-02$ & 6 & 4 & $1.833 \mathrm{E}-01$ & $4.844 \mathrm{E}+01$ & $1.024 \mathrm{E}+07$ \\
\hline & 158215.590 & 166185.470 & $7.260 \mathrm{E}-02$ & 4 & 4 & $3.256 \mathrm{E}-02$ & $5.383 \mathrm{E}+\infty$ & $1.379 \mathrm{E}+06$ \\
\hline & 158215.590 & 166151.610 & $7.230 \mathrm{E}-02$ & 4 & 2 & $1.621 \mathrm{E}-01$ & $2.691 \mathrm{E}+01$ & $1.362 \mathrm{E}+07$ \\
\hline \multirow[t]{4}{*}{$\mathrm{w}^{2} \mathrm{D}^{\circ} \rightarrow \mathrm{h}^{2} \mathrm{P}^{\circ}$} & 0.2762 & 0.2017 & $7.445 \mathrm{E}-02$ & 10 & 6 & $1.411 \mathrm{E}-01$ & $5.687 E+01$ & $1.047 \mathrm{E}+07$ \\
\hline & 158715.460 & 166862.710 & $7.430 \mathrm{E}-02$ & 6 & 4 & $1.408 \mathrm{E}-01$ & $3.412 \mathrm{E}+01$ & $9.368 \mathrm{E}+06$ \\
\hline & 158215.590 & 166862.710 & $7.880 \mathrm{E}-02$ & 4 & 4 & $2.490 \mathrm{E}-02$ & $3.791 \mathrm{E}+\infty 0$ & $1.242 \mathrm{E}+06$ \\
\hline & 158215.590 & 166331.190 & $7.390 \mathrm{E}-02$ & 4 & 2 & $1.167 \mathrm{E}-01$ & $1.896 \mathrm{E}+01$ & $1.024 \mathrm{E}+07$ \\
\hline \multirow[t]{4}{*}{$\mathrm{w}^{2} \mathrm{D}^{\circ} \rightarrow \mathrm{i}^{2} \mathrm{P}^{\circ}$} & 0.2762 & 0.1765 & $9.970 \mathrm{E}-02$ & 10 & 6 & $1.476 \mathrm{E}-04$ & $4.442 \mathrm{E}-02$ & $1.964 \mathrm{E}+04$ \\
\hline & 158715.460 & 169340.390 & $9.680 \mathrm{E}-02$ & 6 & 4 & $1.433 \mathrm{E}-04$ & $2.665 \mathrm{E}-02$ & $1.618 \mathrm{E}+04$ \\
\hline & 158215.590 & 169340.390 & $1.013 \mathrm{E}-01$ & 4 & 4 & $2.500 \mathrm{E}-05$ & $2.962 \mathrm{E}-03$ & $2.061 \mathrm{E}+03$ \\
\hline & 158215.590 & 169687.520 & $1.045 \mathrm{E}-01$ & 4 & 2 & $1.290 \mathrm{E}-04$ & $1.481 \mathrm{E}-02$ & $2.262 \mathrm{E}+04$ \\
\hline \multirow[t]{4}{*}{$w^{2} D^{0} \rightarrow j^{2} P^{e}$} & 0.2762 & 0.1342 & $1.420 \mathrm{E}-01$ & 10 & 6 & $3.946 \mathrm{E}-02$ & $8.336 \mathrm{E}+00$ & $1.065 \mathrm{E}+07$ \\
\hline & 158715.460 & 174274.520 & $1.418 \mathrm{E}-01$ & 6 & 4 & $3.940 \mathrm{E}-02$ & $5.001 E+00$ & $9.545 \mathrm{E}+06$ \\
\hline & 158215.590 & 174274.520 & $1.463 \mathrm{E}-01$ & 4 & 4 & $6.775 \mathrm{E}-03$ & $5.557 \mathrm{E}-01$ & $1.165 \mathrm{E}+06$ \\
\hline & 158215.590 & 173749.010 & $1.415 \mathrm{E}-01$ & 4 & 2 & $3.276 \mathrm{E}-02$ & $2.779 E+00$ & $1.054 \mathrm{E}+07$ \\
\hline \multirow[t]{5}{*}{$w^{2} D^{\circ} \rightarrow f^{2} D^{e}$} & 0.2762 & 0.2673 & $8.917 \mathrm{E}-03$ & 10 & 10 & $2.494 \mathrm{E}-02$ & $8.391 E+01$ & $1.593 \mathrm{E}+04$ \\
\hline & 158715.460 & 159492.830 & $7.100 \mathrm{E}-03$ & 6 & 6 & $1.854 \mathrm{E}-02$ & $4.699 \mathrm{E}+01$ & $7.505 \mathrm{E}+03$ \\
\hline & 158715.460 & 159495.940 & $7.100 \mathrm{E}-03$ & 6 & 4 & $1.324 \mathrm{E}-03$ & $3.357 \mathrm{E}+00$ & $8.041 E+02$ \\
\hline & 158215.590 & 159492.830 & $1.160 \mathrm{E}-02$ & 4 & 6 & $3.245 \mathrm{E}-03$ & $3.357 E+00$ & $2.338 \mathrm{E}+03$ \\
\hline & 158215.590 & 159495.940 & $1.160 \mathrm{E}-02$ & 4 & 4 & $2.920 \mathrm{E}-02$ & $3.021 \mathrm{E}+01$ & $3.156 \mathrm{E}+04$ \\
\hline \multirow[t]{5}{*}{$w^{2} D^{\circ} \rightarrow g^{2} D^{e}$} & 0.2762 & 0.2469 & $2.934 \mathrm{E}-02$ & 10 & 10 & $3.468 \mathrm{E}-03$ & $3.546 \mathrm{E}+00$ & $2.398 \mathrm{E}+04$ \\
\hline & 158715.460 & 161733.100 & $2.750 \mathrm{E}-02$ & 6 & 6 & $3.034 \mathrm{E}-03$ & $1.986 \mathrm{E}+00$ & $1.843 \mathrm{E}+04$ \\
\hline & 158715.460 & 161737.990 & $2.760 \mathrm{E}-02$ & 6 & 4 & $2.175 \mathrm{E}-04$ & $1.419 E-01$ & $1.996 \mathrm{E}+03$ \\
\hline & 158215.590 & 161733.100 & $3.200 \mathrm{E}-02$ & 4 & 6 & $3.783 \mathrm{E}-04$ & $1.419 \mathrm{E}-01$ & $2.074 \mathrm{E}+03$ \\
\hline & 158215.590 & 161737.990 & $3.210 \mathrm{E}-02$ & 4 & 4 & $3.415 \mathrm{E}-03$ & $1.277 \mathrm{E}+00$ & $2.827 \mathrm{E}+04$ \\
\hline \multirow[t]{5}{*}{$w^{2} D^{\circ} \rightarrow b^{2} D^{e}$} & 0.2762 & 0.1944 & $8.181 \mathrm{E}-02$ & 10 & 10 & $3.882 \mathrm{E}-02$ & $1.424 \mathrm{E}+01$ & $2.087 \mathrm{E}+06$ \\
\hline & 158715.460 & 167506.390 & $8.010 \mathrm{E}-02$ & 6 & 6 & $3.548 \mathrm{E}-02$ & $7.972 \mathrm{E}+00$ & $1.828 \mathrm{E}+06$ \\
\hline & 158715.460 & 167472.420 & $7.980 \mathrm{E}-02$ & 6 & 4 & $2.524 \mathrm{E}-03$ & $5.694 \mathrm{E}-01$ & $1.937 \mathrm{E}+05$ \\
\hline & 158215.590 & 167506.390 & $8.460 \mathrm{E}-02$ & 4 & 6 & $4.014 \mathrm{E}-03$ & $5.694 \mathrm{E}-01$ & $1.539 \mathrm{E}+05$ \\
\hline & 158215.590 & 167472.420 & $8.430 \mathrm{E}-02$ & 4 & 4 & $3.600 \mathrm{E}-02$ & $5.125 E+00$ & $2.055 \mathrm{E}+06$ \\
\hline
\end{tabular}


Table IV. Continued

\begin{tabular}{|c|c|c|c|c|c|c|c|c|}
\hline Transition & $\begin{array}{l}E_{i} \\
\mathrm{~cm}^{-1}\end{array}$ & $\begin{array}{l}E_{f} \\
\mathrm{~cm}^{-1}\end{array}$ & $\begin{array}{l}E_{f i} \\
\text { Ry }\end{array}$ & $g_{i}$ & $g_{f}$ & $f_{i f}$ & $S$ & $\begin{array}{l}A_{f i} \\
\mathrm{~s}^{-1}\end{array}$ \\
\hline \multirow[t]{5}{*}{$\mathrm{w}^{2} \mathrm{D}^{\circ} \rightarrow \mathrm{i}^{2} \mathrm{D}^{\mathrm{c}}$} & 0.2762 & 0.1717 & $1.045 \mathrm{E}-01$ & 10 & 10 & $5.670 \mathrm{E}-02$ & $1.628 \mathrm{E}+01$ & $4.969 \mathrm{E}+06$ \\
\hline & 158715.460 & 170015.230 & $1.030 \mathrm{E}-01$ & 6 & 6 & $5.218 \mathrm{E}-02$ & $9.120 \mathrm{E}+00$ & $4.447 \mathrm{E}+06$ \\
\hline & 158715.460 & 169922.270 & $1.021 E-01$ & 6 & 4 & $3.695 \mathrm{E}-03$ & $6.514 \mathrm{E}-01$ & $4.641 \mathrm{E}+05$ \\
\hline & 158215.590 & 170015.230 & $1.075 \mathrm{E}-01$ & 4 & 6 & $5.835 E-03$ & $6.514 \mathrm{E}-01$ & $3.611 \mathrm{E}+05$ \\
\hline & 158215.590 & 169922.270 & $1.066 \mathrm{E}-01$ & 4 & 4 & $5.208 \mathrm{E}-02$ & $5.863 E+\infty$ & $4.754 E+06$ \\
\hline \multirow[t]{5}{*}{$w^{2} D^{o} \rightarrow j^{2} D^{e}$} & 0.2762 & 0.1071 & $1.691 \mathrm{E}-01$ & 10 & 10 & $2.103 \mathrm{E}-04$ & $3.730 \mathrm{E}-02$ & $4.833 \mathrm{E}+04$ \\
\hline & 158715.460 & 177076.280 & $1.673 \mathrm{E}-01$ & 6 & 6 & $1.941 \mathrm{E}-04$ & $2.089 \mathrm{E}-02$ & $4.365 \mathrm{E}+04$ \\
\hline & 158715.460 & 177077.020 & $1.673 \mathrm{E}-01$ & 6 & 4 & $1.387 \mathrm{E}-05$ & $1.492 \mathrm{E}-03$ & $4.676 \mathrm{E}+03$ \\
\hline & 158215.590 & 177076.280 & $1.718 \mathrm{E}-01$ & 4 & 6 & $2.136 \mathrm{E}-05$ & $1.492 \mathrm{E}-03$ & $3.376 \mathrm{E}+03$ \\
\hline & 158215.590 & 177077.020 & $1.718 \mathrm{E}-01$ & 4 & 4 & $1.922 \mathrm{E}-04$ & $1.343 \mathrm{E}-02$ & $4.558 \mathrm{E}+04$ \\
\hline \multirow[t]{5}{*}{$w^{2} D^{\circ} \rightarrow k^{2} D^{\circ}$} & 0.2762 & 0.0381 & $2.381 \mathrm{E}-01$ & 10 & 10 & $1.794 \mathrm{E}-03$ & $2.260 \mathrm{E}-01$ & $8.167 \mathrm{E}+05$ \\
\hline & 158715.460 & 184642.760 & $2.363 \mathrm{E}-01$ & 6 & 6 & $1.661 \mathrm{E}-03$ & $1.266 \mathrm{E}-01$ & $7.452 E+05$ \\
\hline & 158715.460 & 184642.760 & $2.363 \mathrm{E}-01$ & 6 & 4 & $1.187 \mathrm{E}-04$ & $9.040 \mathrm{E}-03$ & $7.984 \mathrm{E}+04$ \\
\hline & 158215.590 & 184642.760 & $2.408 \mathrm{E}-01$ & 4 & 6 & $1.814 \mathrm{E}-04$ & $9.040 \mathrm{E}-03$ & $5.633 \mathrm{E}+04$ \\
\hline & 158215.590 & 184642.760 & $2.408 \mathrm{E}-01$ & 4 & 4 & $1.633 \mathrm{E}-03$ & $8.136 \mathrm{E}-02$ & $7.604 \mathrm{E}+05$ \\
\hline \multirow[t]{4}{*}{$\mathrm{w}^{2} \mathrm{D}^{\circ} \rightarrow \mathrm{d}^{2} \mathrm{~F}^{\mathrm{e}}$} & 0.2762 & 0.2236 & $5.264 \mathrm{E}-02$ & 10 & 14 & $2.727 \mathrm{E}-01$ & $1.554 \mathrm{E}+02$ & $4.336 \mathrm{E}+06$ \\
\hline & 158715.460 & 164337.610 & $5.130 \mathrm{E}-02$ & 6 & 8 & $2.531 \mathrm{E}-01$ & $8.880 \mathrm{E}+01$ & $4.012 \mathrm{E}+06$ \\
\hline & 158715.460 & 164232.360 & $5.030 \mathrm{E}-02$ & 6 & 6 & $1.241 \mathrm{E}-02$ & $4.440 \mathrm{E}+00$ & $2.522 \mathrm{E}+05$ \\
\hline & 158215.590 & 164232.360 & $5.480 \mathrm{E}-02$ & 4 & 6 & $2.839 \mathrm{E}-01$ & $6.216 \mathrm{E}+01$ & $4.565 \mathrm{E}+06$ \\
\hline \multirow[t]{4}{*}{$\mathrm{w}^{2} \mathrm{D}^{\circ} \rightarrow \mathrm{e}^{2} \mathrm{~F}^{e}$} & 0.2762 & 0.1759 & $1.003 \mathrm{E}-01$ & 10 & 14 & $5.035 \mathrm{E}-01$ & $1.506 \mathrm{E}+02$ & $2.907 \mathrm{E}+07$ \\
\hline & 158715.460 & 169702.320 & $1.001 \mathrm{E}-01$ & 6 & 8 & $4.786 \mathrm{E}-01$ & $8.605 \mathrm{E}+01$ & $2.889 \mathrm{E}+07$ \\
\hline & 158715.460 & 169283.540 & $9.630 \mathrm{E}-02$ & 6 & 6 & $2.302 \mathrm{E}-02$ & $4.303 \mathrm{E}+00$ & $1.715 \mathrm{E}+06$ \\
\hline & 158215.590 & 169283.540 & $1.008 \mathrm{E}-01$ & 4 & 6 & $5.060 \mathrm{E}-01$ & $6.024 \mathrm{E}+01$ & $2.753 E+07$ \\
\hline \multirow[t]{4}{*}{$\mathrm{z}^{2} \mathrm{~F}^{\circ} \rightarrow \mathrm{d}^{2} \mathrm{D}^{\circ}$} & 0.4424 & 0.4079 & $3.446 \mathrm{E}-02$ & 14 & 10 & $3.992 \mathrm{E}-03$ & $4.865 \mathrm{E}+\infty 0$ & $5.330 \mathrm{E}+04$ \\
\hline & 140319.230 & 144009.420 & $3.360 \mathrm{E}-02$ & 8 & 6 & $3.892 \mathrm{E}-03$ & $2.780 \mathrm{E}+\infty 0$ & $4.706 \mathrm{E}+04$ \\
\hline & 140230.100 & 144009.420 & $3.440 \mathrm{E}-02$ & 6 & 6 & $2.656 \mathrm{E}-04$ & $1.390 \mathrm{E}-01$ & $2.525 \mathrm{E}+03$ \\
\hline & 140230.100 & 144142.160 & $3.560 \mathrm{E}-02$ & 6 & 4 & $3.849 E-03$ & $1.946 \mathrm{E}+00$ & $5.877 \mathrm{E}+04$ \\
\hline \multirow[t]{4}{*}{$\mathrm{z}^{2} \mathrm{~F}^{0} \rightarrow \mathrm{e}^{2} \mathrm{D}^{\mathrm{e}}$} & 0.4424 & 0.3639 & $7.847 \mathrm{E}-02$ & 14 & 10 & $2.789 \mathrm{E}-03$ & $1.493 \mathrm{E}+00$ & $1.931 \mathrm{E}+05$ \\
\hline & 140319.230 & 148886.570 & $7.810 \mathrm{E}-02$ & 8 & 6 & $2.776 \mathrm{E}-03$ & $8.530 \mathrm{E}-01$ & $1.813 \mathrm{E}+05$ \\
\hline & 140230.100 & 148886.570 & $7.890 \mathrm{E}-02$ & 6 & 6 & $1.869 \mathrm{E}-04$ & $4.265 \mathrm{E}-02$ & $9.348 \mathrm{E}+03$ \\
\hline & 140230.100 & 148900.910 & $7.900 \mathrm{E}-02$ & 6 & 4 & $2.621 \mathrm{E}-03$ & $5.971 \mathrm{E}-01$ & $1.970 \mathrm{E}+05$ \\
\hline \multirow[t]{4}{*}{$z^{2} F^{o} \rightarrow f^{2} D^{e}$} & 0.4424 & 0.2673 & $1.751 \mathrm{E}-01$ & 14 & 10 & $3.240 \mathrm{E}-03$ & $7.773 \mathrm{E}-01$ & $1.117 \mathrm{E}+06$ \\
\hline & 140319.230 & 159492.830 & $1.747 \mathrm{E}-01$ & 8 & 6 & $3.233 E-03$ & $4.442 E-01$ & $1.057 \mathrm{E}+06$ \\
\hline & 140230.100 & 159492.830 & $1.755 \mathrm{E}-01$ & 6 & 6 & $2.165 \mathrm{E}-04$ & $2.221 \mathrm{E}-02$ & $5.357 \mathrm{E}+04$ \\
\hline & 140230.100 & 159495.940 & $1.755 \mathrm{E}-01$ & 6 & 4 & $3.032 \mathrm{E}-03$ & $3.109 \mathrm{E}-01$ & $1.125 \mathrm{E}+06$ \\
\hline \multirow[t]{4}{*}{$\mathrm{z}^{2} \mathrm{~F}^{\circ} \rightarrow \mathrm{g}^{2} \mathrm{D}^{\mathrm{e}}$} & 0.4424 & 0.2469 & $1.955 \mathrm{E}-01$ & 14 & 10 & $2.070 E-01$ & $4.447 \mathrm{E}+01$ & $8.898 E+07$ \\
\hline & 140319.230 & 161733.100 & $1.951 \mathrm{E}-01$ & 8 & 6 & $2.066 \mathrm{E}-01$ & $2.541 \mathrm{E}+01$ & $8.422 \mathrm{E}+07$ \\
\hline & 140230.100 & 161733.100 & $1.959 \mathrm{E}-01$ & 6 & 6 & $1.383 E-02$ & $1.271 \mathrm{E}+\infty 0$ & $4.263 \mathrm{E}+06$ \\
\hline & 140230.100 & 161737.990 & $1.960 \mathrm{E}-01$ & 6 & 4 & $1.937 \mathrm{E}-01$ & $1.779 \mathrm{E}+01$ & $8.966 \mathrm{E}+07$ \\
\hline \multirow[t]{4}{*}{$\mathrm{z}^{2} \mathrm{~F}^{\circ} \rightarrow \mathrm{b}^{2} \mathrm{D}^{\mathrm{e}}$} & 0.4424 & 0.1944 & $2.480 \mathrm{E}-01$ & 14 & 10 & $1.611 \mathrm{E}-02$ & $2.728 \mathrm{E}+00$ & $1.114 \mathrm{E}+07$ \\
\hline & 140319.230 & 167506.390 & $2.477 \mathrm{E}-01$ & 8 & 6 & $1.609 \mathrm{E}-02$ & $1.559 \mathrm{E}+00$ & $1.057 \mathrm{E}+07$ \\
\hline & 140230.100 & 167506.390 & $2.485 \mathrm{E}-01$ & 6 & 6 & $1.076 \mathrm{E}-03$ & $7.795 E-02$ & $5.338 \mathrm{E}+05$ \\
\hline & 140230.100 & 167472.420 & $2.482 \mathrm{E}-01$ & 6 & 4 & $1.505 \mathrm{E}-02$ & $1.091 \mathrm{E}+00$ & $1.117 \mathrm{E}+07$ \\
\hline \multirow[t]{4}{*}{$\mathrm{z}^{2} \mathrm{~F}^{\circ} \rightarrow \mathrm{i}^{2} \mathrm{D}^{\mathrm{e}}$} & 0.4424 & 0.1717 & $2.706 \mathrm{E}-01$ & 14 & 10 & $1.125 \mathrm{E}-03$ & $1.745 \mathrm{E}-01$ & $9.261 \mathrm{E}+05$ \\
\hline & 140319.230 & 170015.230 & $2.706 \mathrm{E}-01$ & 8 & 6 & $1.124 \mathrm{E}-03$ & $9.973 \mathrm{E}-02$ & $8.818 \mathrm{E}+05$ \\
\hline & 140230.100 & 170015.230 & $2.714 \mathrm{E}-01$ & 6 & 6 & $7.519 E-05$ & $4.987 \mathrm{E}-03$ & $4.448 \mathrm{E}+04$ \\
\hline & 140230.100 & 169922.270 & $2.705 \mathrm{E}-01$ & 6 & 4 & $1.049 \mathrm{E}-03$ & $6.981 \mathrm{E}-02$ & $9.249 \mathrm{E}+05$ \\
\hline \multirow[t]{4}{*}{$\mathrm{z}^{2} \mathrm{~F}^{0} \rightarrow \mathrm{j}^{2} \mathrm{D}^{\mathrm{e}}$} & 0.4424 & 0.1071 & $3.353 \mathrm{E}-01$ & 14 & 10 & $2.629 E-02$ & $3.293 \mathrm{E}+00$ & $3.323 \mathrm{E}+07$ \\
\hline & 140319.230 & 177076.280 & $3.349 \mathrm{E}-01$ & 8 & 6 & $2.625 \mathrm{E}-02$ & $1.882 \mathrm{E}+00$ & $3.154 \mathrm{E}+07$ \\
\hline & 140230.100 & 177076.280 & $3.357 \mathrm{E}-01$ & 6 & 6 & $1.755 E-03$ & $9.408 \mathrm{E}-02$ & $1.588 \mathrm{E}+06$ \\
\hline & 140230.100 & 177077.020 & $3.357 \mathrm{E}-01$ & 6 & 4 & $2.456 \mathrm{E}-02$ & $1.317 \mathrm{E}+00$ & $3.335 \mathrm{E}+07$ \\
\hline
\end{tabular}


Table IV. Continued

\begin{tabular}{|c|c|c|c|c|c|c|c|c|}
\hline Transition & $\begin{array}{l}E_{\mathrm{i}} \\
\mathrm{cm}^{-1}\end{array}$ & $\begin{array}{l}E_{f} \\
\mathrm{~cm}^{-1}\end{array}$ & $\begin{array}{l}E_{f i} \\
\mathrm{Ry}\end{array}$ & $g_{i}$ & $g_{f}$ & $f_{i f}$ & $S$ & $\begin{array}{l}A_{f i} \\
\mathrm{~s}^{-1}\end{array}$ \\
\hline \multirow[t]{4}{*}{$\mathrm{z}^{2} \mathrm{~F}^{\mathrm{e}} \rightarrow \mathrm{k}^{2} \mathrm{D}^{\mathrm{c}}$} & 0.4424 & 0.0381 & $4.043 E-01$ & 14 & 10 & $3.829 \mathrm{E}-06$ & $3.978 \mathrm{E}-04$ & $7.036 \mathrm{E}+03$ \\
\hline & 140319.230 & 184642.760 & $4.039 \mathrm{E}-01$ & 8 & 6 & $3.825 \mathrm{E}-06$ & $2.273 \mathrm{E}-04$ & $6.684 E+03$ \\
\hline & 140230.100 & 184642.760 & $4.047 \mathrm{E}-01$ & 6 & 6 & $2.555 \mathrm{E}-07$ & $1.137 \mathrm{E}-05$ & $3.362 \mathrm{E}+02$ \\
\hline & 140230.100 & 184642.760 & $4.047 \mathrm{E}-01$ & 6 & 4 & $3.578 \mathrm{E}-06$ & $1.591 \mathrm{E}-04$ & $7.060 \mathrm{E}+03$ \\
\hline \multirow[t]{5}{*}{$\mathrm{z}^{2} \mathrm{~F}^{\mathrm{o}} \rightarrow \mathrm{c}^{2} \mathrm{~F}^{\mathrm{e}}$} & 0.4424 & 0.2955 & $1.469 \mathrm{E}-01$ & 14 & 14 & $4.824 \mathrm{E}-02$ & $1.380 \mathrm{E}+01$ & $8.358 \mathrm{E}+06$ \\
\hline & 140319.230 & 156604.170 & $1.484 \mathrm{E}-01$ & 8 & 8 & $4.701 \mathrm{E}-02$ & $7.602 \mathrm{E}+00$ & $8.315 E+06$ \\
\hline & 140319.230 & 156121.700 & $1.440 \mathrm{E}-01$ & 8 & 6 & $1.689 \mathrm{E}-03$ & $2.816 \mathrm{E}-01$ & $3.752 \mathrm{E}+05$ \\
\hline & 140230.100 & 156604.170 & $1.492 \mathrm{E}-01$ & 6 & 8 & $2.334 E-03$ & $2.816 \mathrm{E}-01$ & $3.130 \mathrm{E}+05$ \\
\hline & 140230.100 & 156121.700 & $1.448 \mathrm{E}-01$ & 6 & 6 & $4.576 \mathrm{E}-02$ & $5.688 \mathrm{E}+00$ & $7.706 \mathrm{E}+06$ \\
\hline \multirow[t]{5}{*}{$\mathrm{z}^{2} \mathrm{~F}^{\circ} \rightarrow \mathrm{d}^{2} \mathrm{~F}^{e}$} & 0.4424 & 0.2236 & $2.188 \mathrm{E}-01$ & 14 & 14 & $1.446 \mathrm{E}-01$ & $2.776 \mathrm{E}+01$ & $5.562 \mathrm{E}+07$ \\
\hline & 140319.230 & 164337.610 & $2.189 \mathrm{E}-01$ & 8 & 8 & $1.395 \mathrm{E}-01$ & $1.530 \mathrm{E}+01$ & $5.370 \mathrm{E}+07$ \\
\hline & 140319.230 & 164232.360 & $2.179 \mathrm{E}-01$ & 8 & 6 & $5.144 \mathrm{E}-03$ & $5.666 \mathrm{E}-01$ & $2.616 \mathrm{E}+06$ \\
\hline & 140230.100 & 164337.610 & $2.197 \mathrm{E}-01$ & 6 & 8 & $6.915 \mathrm{E}-03$ & $5.666 \mathrm{E}-01$ & $2.011 E+06$ \\
\hline & 140230.100 & 164232.360 & $2.187 \mathrm{E}-01$ & 6 & 6 & $1.391 \mathrm{E}-01$ & $1.144 \mathrm{E}+01$ & $5.342 \mathrm{E}+07$ \\
\hline \multirow[t]{5}{*}{$z^{2} F^{0} \rightarrow e^{2} F^{e}$} & 0.4424 & 0.1759 & $2.665 \mathrm{E}-01$ & 14 & 14 & $1.619 \mathrm{E}-02$ & $2.551 \mathrm{E}+00$ & $9.231 \mathrm{E}+06$ \\
\hline & 140319.230 & 169702.320 & $2.677 \mathrm{E}-01$ & 8 & 8 & $1.568 \mathrm{E}-02$ & $1.406 \mathrm{E}+\infty 0$ & $9.025 \mathrm{E}+06$ \\
\hline & 140319.230 & 169283.540 & $2.639 \mathrm{E}-01$ & 8 & 6 & $5.725 \mathrm{E}-04$ & $5.206 \mathrm{E}-02$ & $4.270 \mathrm{E}+05$ \\
\hline & 140230.100 & 169702.320 & $2.685 \mathrm{E}-01$ & 6 & 8 & $7.766 \mathrm{E}-04$ & $5.206 \mathrm{E}-02$ & $3.373 E+05$ \\
\hline & 140230.100 & 169283.540 & $2.647 \mathrm{E}-01$ & 6 & 6 & $1.547 E-02$ & $1.052 \mathrm{E}+00$ & $8.704 E+06$ \\
\hline \multirow[t]{4}{*}{$\mathrm{z}^{2} \mathrm{~F}^{\circ} \rightarrow \mathrm{b}^{2} \mathrm{G}^{\mathrm{e}}$} & 0.4424 & 0.2241 & $2.182 \mathrm{E}-01$ & 14 & 18 & $7.934 \mathrm{E}-01$ & $1.527 \mathrm{E}+02$ & $2.361 \mathrm{E}+08$ \\
\hline & 140319.230 & 164268.790 & $2.182 \mathrm{E}-01$ & 8 & 10 & $7.712 \mathrm{E}-01$ & $8.483 \mathrm{E}+01$ & $2.359 \mathrm{E}+08$ \\
\hline & 140319.230 & 164181.170 & $2.174 \mathrm{E}-01$ & 8 & 8 & $2.195 \mathrm{E}-02$ & $2.424 \mathrm{E}+00$ & $8.334 \mathrm{E}+06$ \\
\hline & 140230.100 & 164181.170 & $2.182 \mathrm{E}-01$ & 6 & 8 & $7.932 \mathrm{E}-01$ & $6.544 \mathrm{E}+01$ & $2.275 \mathrm{E}+08$ \\
\hline \multirow[t]{4}{*}{$\mathrm{z}^{2} \mathrm{~F}^{0} \rightarrow \mathrm{c}^{2} \mathrm{G}^{e}$} & 0.4424 & 0.0967 & $3.456 \mathrm{E}-01$ & 14 & 18 & $7.745 \mathrm{E}-02$ & $9.411 \mathrm{E}+00$ & $5.780 \mathrm{E}+07$ \\
\hline & 140319.230 & 178240.640 & $3.455 \mathrm{E}-01$ & 8 & 10 & $7.527 \mathrm{E}-02$ & $5.228 \mathrm{E}+00$ & $5.773 \mathrm{E}+07$ \\
\hline & 140319.230 & 178173.390 & $3.449 \mathrm{E}-01$ & 8 & 8 & $2.147 \mathrm{E}-03$ & $1.494 \mathrm{E}-01$ & $2.051 E+06$ \\
\hline & 140230.100 & 178173.390 & $3.457 \mathrm{E}-01$ & 6 & 8 & $7.746 \mathrm{E}-02$ & $4.033 \mathrm{E}+\infty 0$ & $5.577 \mathrm{E}+07$ \\
\hline \multirow[t]{4}{*}{$\mathrm{y}^{2} \mathrm{~F}^{\circ} \rightarrow \mathrm{i}^{2} \mathrm{D}^{\circ}$} & 0.1856 & 0.1717 & $1.383 \mathrm{E}-02$ & 14 & 10 & $1.725 \mathrm{E}-03$ & $5.238 \mathrm{E}+00$ & $3.709 E+03$ \\
\hline & 168473.480 & 170015.230 & $1.410 \mathrm{E}-02$ & 8 & 6 & $1.758 \mathrm{E}-03$ & $2.993 \mathrm{E}+00$ & $3.744 \mathrm{E}+03$ \\
\hline & 168443.140 & 170015.230 & $1.430 \mathrm{E}-02$ & 6 & 6 & $1.189 \mathrm{E}-04$ & $1.496 \mathrm{E}-01$ & $1.953 E+02$ \\
\hline & 168443.140 & 169922.270 & $1.340 \mathrm{E}-02$ & 6 & 4 & $1.560 \mathrm{E}-03$ & $2.095 \mathrm{E}+00$ & $3.374 E+03$ \\
\hline \multirow[t]{4}{*}{$\mathrm{y}^{2} \mathrm{~F}^{\circ} \rightarrow \mathrm{j}^{2} \mathrm{D}^{\mathrm{e}}$} & 0.1856 & 0.1071 & $7.852 \mathrm{E}-02$ & 14 & 10 & $3.007 \mathrm{E}-01$ & $1.609 \mathrm{E}+02$ & $2.085 \mathrm{E}+07$ \\
\hline & 168473.480 & 177076.280 & $7.840 \mathrm{E}-02$ & 8 & 6 & $3.003 \mathrm{E}-01$ & $9.193 \mathrm{E}+01$ & $1.977 \mathrm{E}+07$ \\
\hline & 168443.140 & 177076.280 & $7.860 \mathrm{E}-02$ & 6 & 6 & $2.007 \mathrm{E}-02$ & $4.597 \mathrm{E}+00$ & $9.960 \mathrm{E}+05$ \\
\hline & 168443.140 & 177077.020 & $7.860 \mathrm{E}-02$ & 6 & 4 & $2.810 \mathrm{E}-01$ & $6.435 E+01$ & $2.092 E+07$ \\
\hline \multirow[t]{4}{*}{$\mathrm{y}^{2} \mathrm{~F}^{\circ} \rightarrow \mathrm{k}^{2} \mathrm{D}^{\circ}$} & 0.1856 & 0.0381 & $1.475 \mathrm{E}-01$ & 14 & 10 & $2.997 \mathrm{E}-06$ & $8.535 \mathrm{E}-04$ & $7.328 \mathrm{E}+02$ \\
\hline & 168473.480 & 184642.760 & $1.474 \mathrm{E}-01$ & 8 & 6 & $2.995 \mathrm{E}-06$ & $4.877 \mathrm{E}-04$ & $6.970 \mathrm{E}+02$ \\
\hline & 168443.140 & 184642.760 & $1.476 \mathrm{E}-01$ & 6 & 6 & $2.000 \mathrm{E}-07$ & $2.438 \mathrm{E}-05$ & $3.499 E+01$ \\
\hline & 168443.140 & 184642.760 & $1.476 \mathrm{E}-01$ & 6 & 4 & $2.799 \mathrm{E}-06$ & $3.414 \mathrm{E}-04$ & $7.348 \mathrm{E}+02$ \\
\hline \multirow[t]{5}{*}{$y^{2} F^{0} \rightarrow e^{2} F^{\bullet}$} & 0.1856 & 0.1759 & $9.681 \mathrm{E}-03$ & 14 & 14 & $6.398 \mathrm{E}-03$ & $2.776 \mathrm{E}+01$ & $4.817 \mathrm{E}+03$ \\
\hline & 168473.480 & 169702.320 & $1.120 \mathrm{E}-02$ & 8 & 8 & $7.138 \mathrm{E}-03$ & $1.530 \mathrm{E}+01$ & $7.192 \mathrm{E}+03$ \\
\hline & 168473.480 & 169283.540 & $7.400 \mathrm{E}-03$ & 8 & 6 & $1.747 \mathrm{E}-04$ & $5.665 \mathrm{E}-01$ & $1.024 E+02$ \\
\hline & 168443.140 & 169702.320 & $1.140 \mathrm{E}-02$ & 6 & 8 & $3.588 \mathrm{E}-04$ & $5.665 \mathrm{E}-01$ & $2.809 \mathrm{E}+02$ \\
\hline & 168443.140 & 169283.540 & $7.600 \mathrm{E}-03$ & 6 & 6 & $4.832 \mathrm{E}-03$ & $1.144 \mathrm{E}+01$ & $2.242 \mathrm{E}+03$ \\
\hline \multirow[t]{4}{*}{$y^{2} F^{\circ} \rightarrow c^{2} G^{e}$} & 0.1856 & 0.0967 & $8.885 \mathrm{E}-02$ & 14 & 18 & $9.545 \mathrm{E}-01$ & $4.512 \mathrm{E}+02$ & $4.708 \mathrm{E}+07$ \\
\hline & 168473.480 & 178240.640 & $8.900 \mathrm{E}-02$ & 8 & 10 & $9.296 \mathrm{E}-01$ & $2.507 \mathrm{E}+02$ & $4.731 E+07$ \\
\hline & 168473.480 & 178173.390 & $8.840 \mathrm{E}-02$ & 8 & 8 & $2.638 \mathrm{E}-02$ & $7.162 E+00$ & $1.656 \mathrm{E}+06$ \\
\hline & 168443.140 & 178173.390 & $8.860 E-02$ & 6 & 8 & $9.518 \mathrm{E}-01$ & $1.934 \mathrm{E}+02$ & $4.501 \mathrm{E}+07$ \\
\hline
\end{tabular}

